


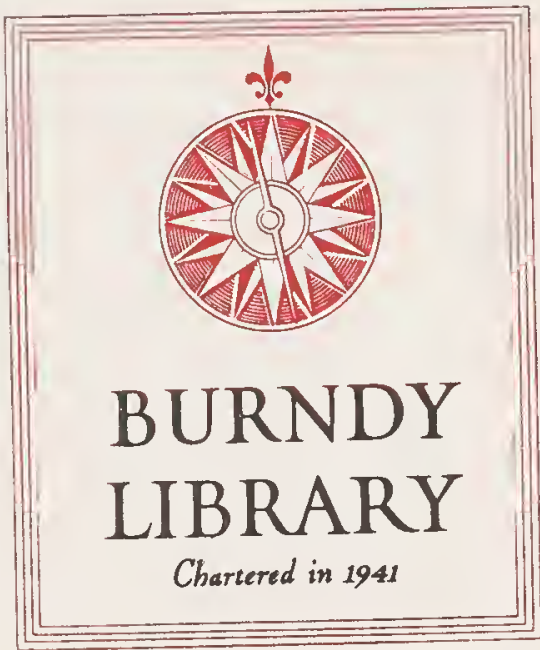

GIFT OF

BERN DIBNER 

(4) 3 - 
$\left(1+w^{2}\right.$ 


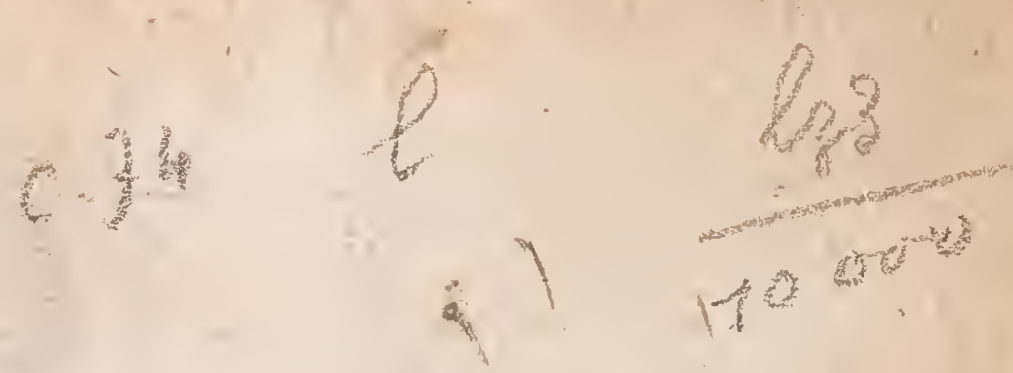

\section{LA FORZA DELLA}

\section{LEVA \\ OPERA \\ $\mathrm{Di}$}

ROMANO FORTVINATO CARAPECCHIA

Consegrata

ALLALTEZZA SEREN:

RAIMONDOPERELLOS Gran Maestro della S.R.G. Prencipe di Malta, e del Gozzo 
A1MOANG

$$
\begin{gathered}
\text { 1.1] } \\
\text { xamo } \\
0
\end{gathered}
$$

GTPANFHE OHAMUH.

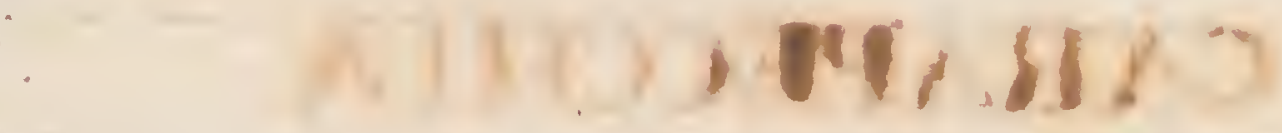

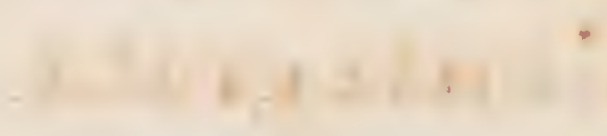

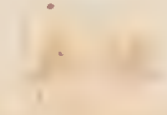

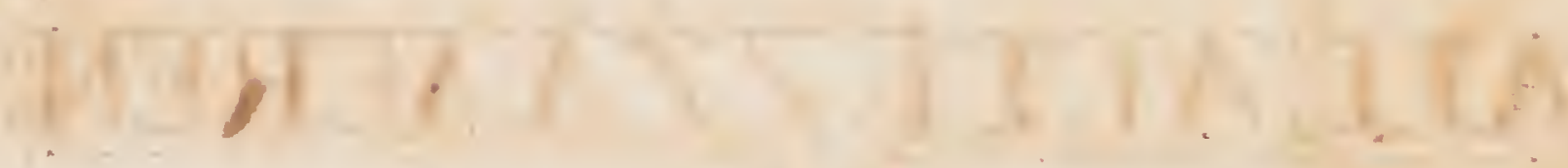

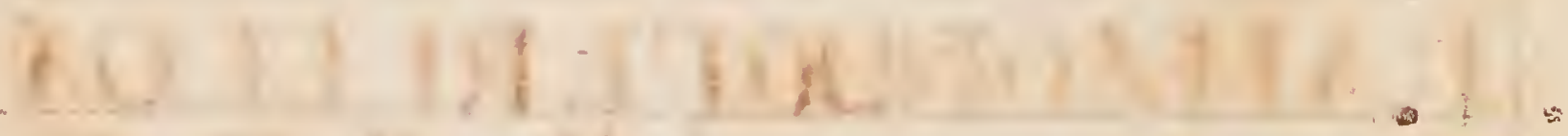

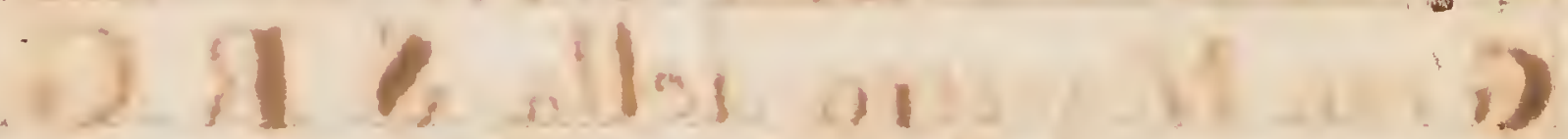

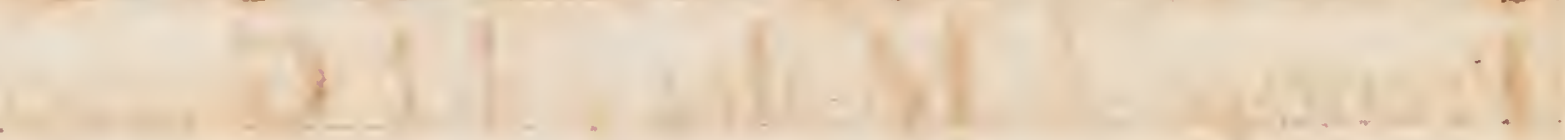




\section{ALTEZZA}
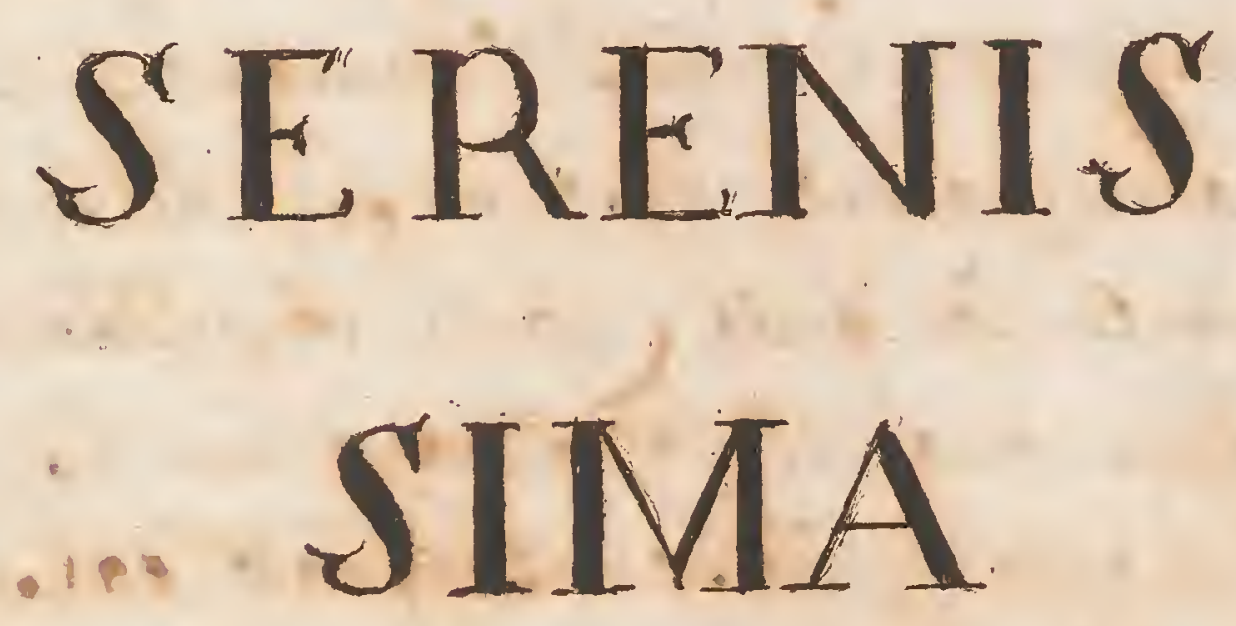

S i cho il Moñou' ammira eo

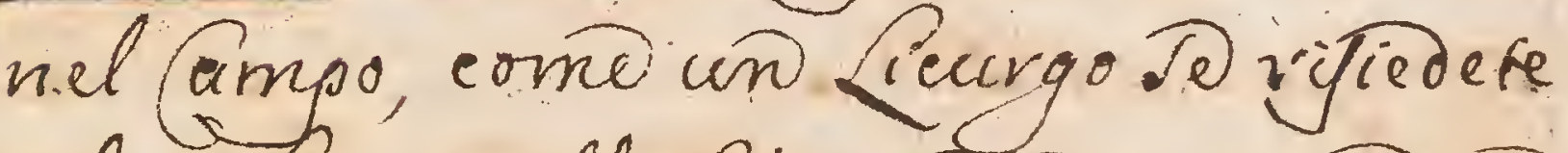
sul Joglio, quello fü grande, uor godept pavimenfo gl atfriousi oi Gande, má con un aucin baggio di pié, che se quello 


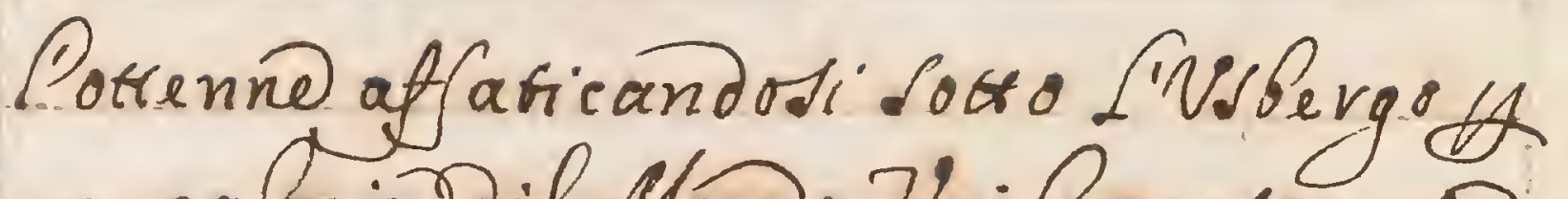
uanagloria del Mondo, Voi R consequifre offerendo it angue thebellave Inferno

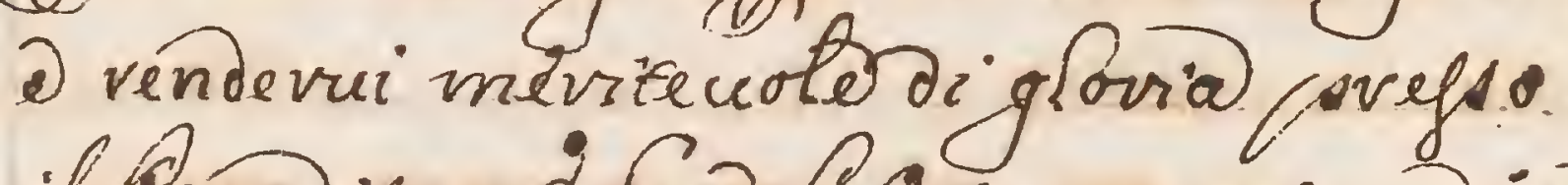
ilfaradiso é Gen Pormano teme in Lioi quel ualore chelo uinse; 0 Religione

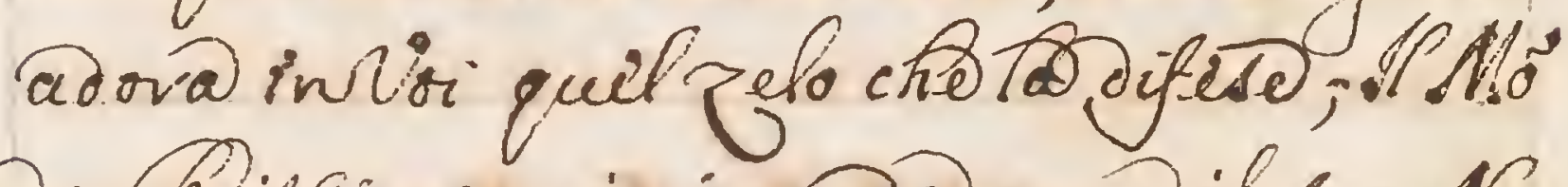
do Tristian uiniquando comeil suo Na: me rutelave; stlo visueglearo dalsusur vo bansi asslausi in sounti alle glo =

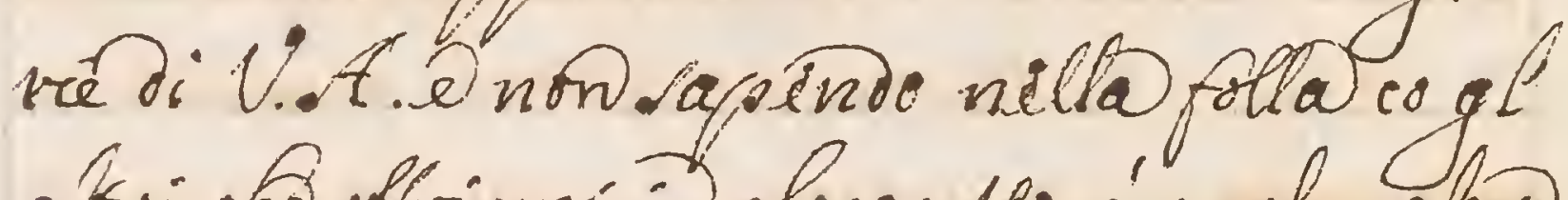
aitri cho offirimi in olocautro a' quel ciabovo áeui pvmandeltagloria uien befura quilla candida fajeio unico sacenobd Arace. Ali visolfi di acrificarrie i Seren: frier un crimoparto deta mial penna

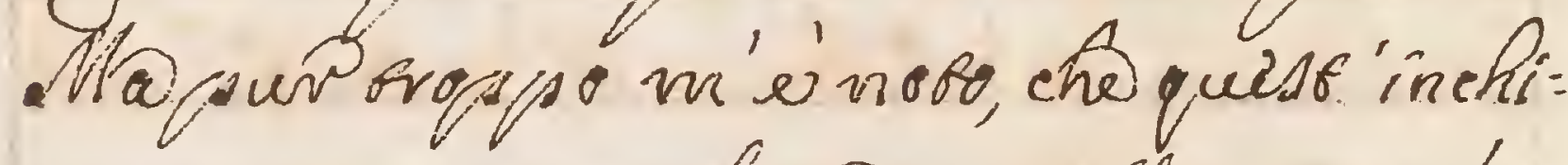

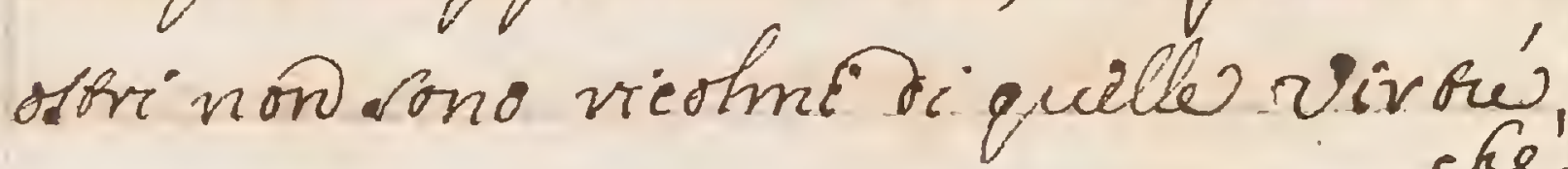
cke' 
MSS $245 B$

RB NMAT

cho libeñ covonano SAnimo Di V.A ma conviene ineoffarnd Pocierba'siquel br: Cenro cho non puedevipe colfera magqiove

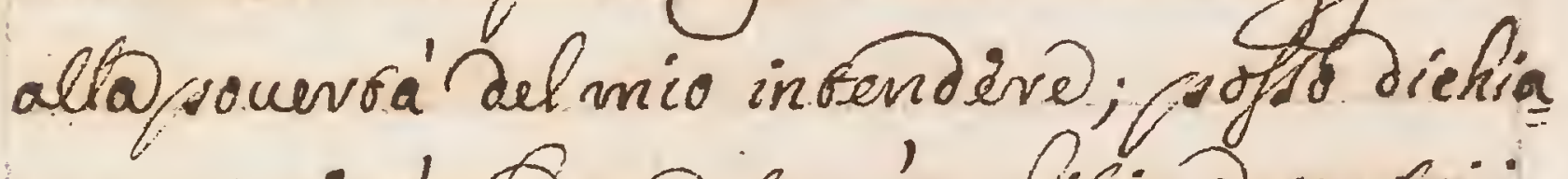
varmi pivo', chenonisens ambifione nutizi

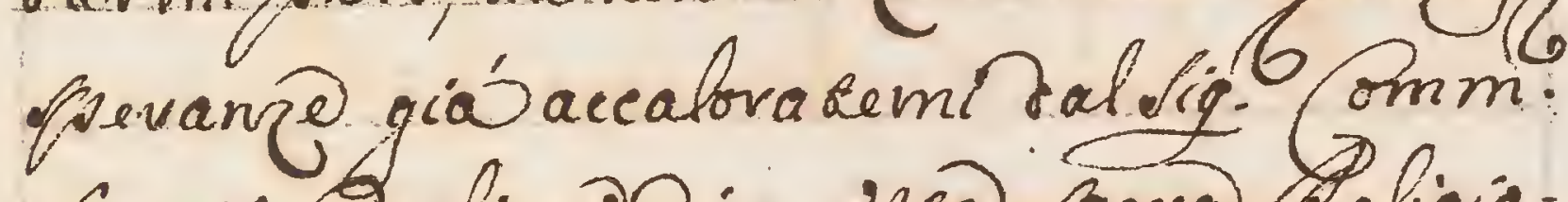
foreti aualievoraiquesoa Revaligio.

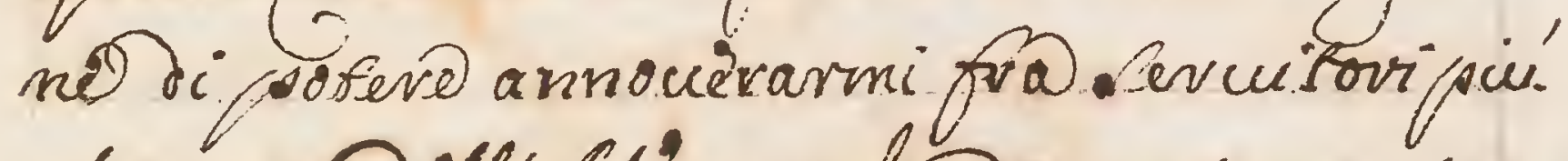
ofrequisi dell'ar. V. propalands mio uantay: gio fambitiondiquisaquia, chossivo Jeĺa quale ebevnamigoró quamo il Monso contideri infronfo driquesto ifro gl"ati delmio nofonjojimo viasetro Q. i.A.

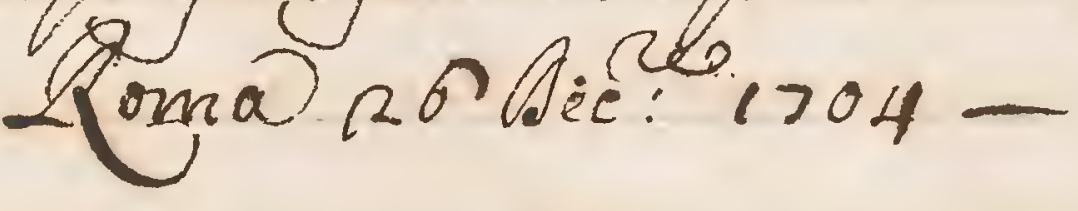

Vino Deciof. is oflig. Sev Lomanoforsunato (avapectia 
f

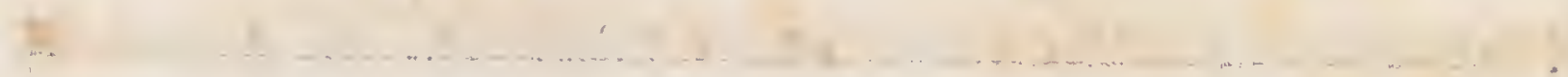

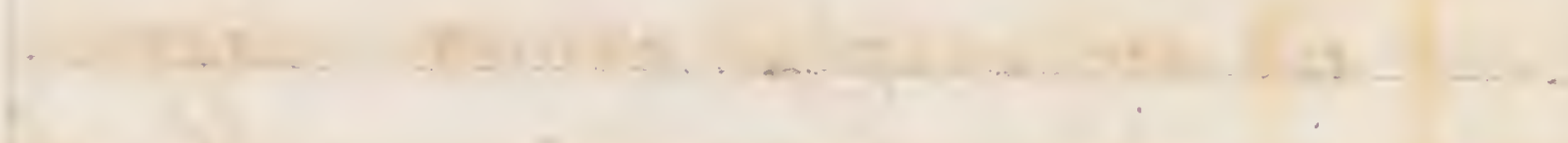

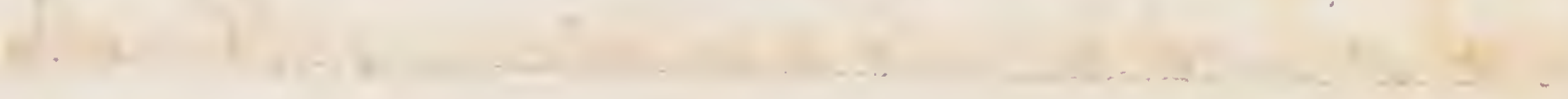
.

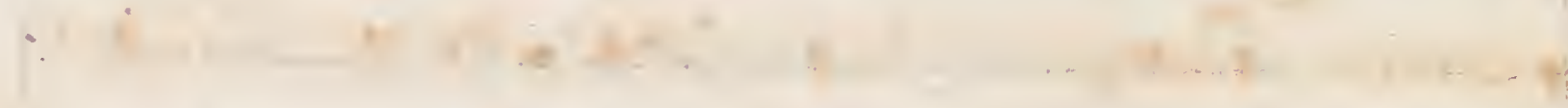

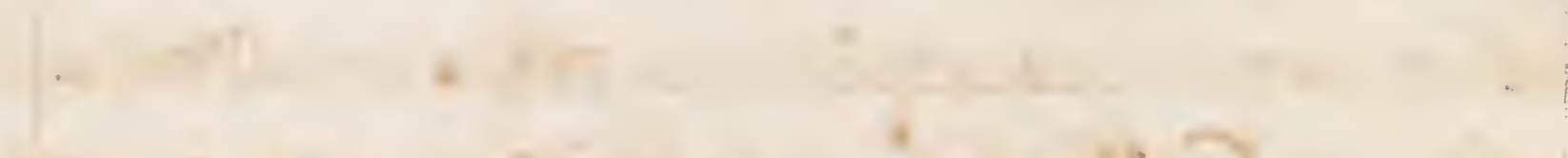

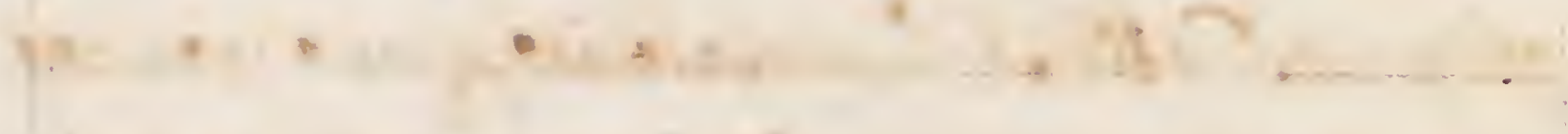

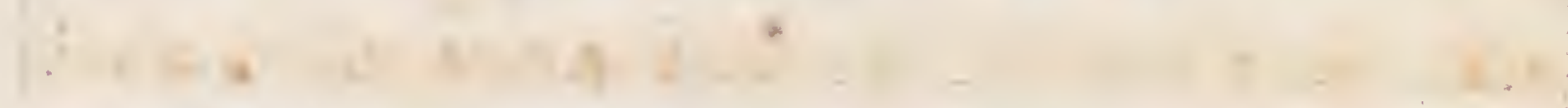

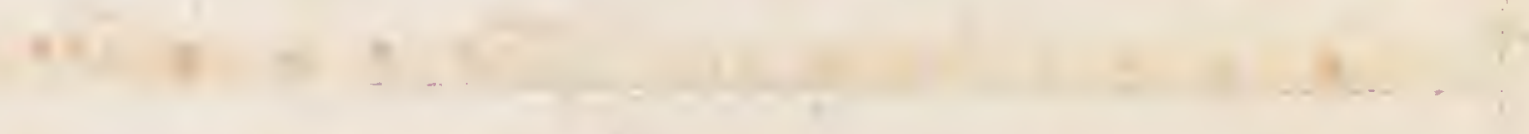

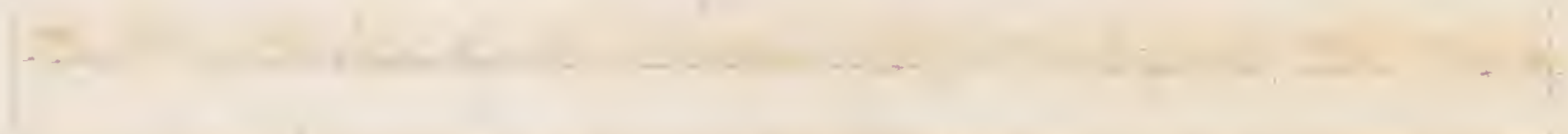
-

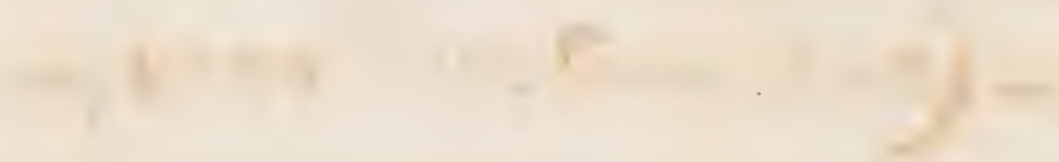
4

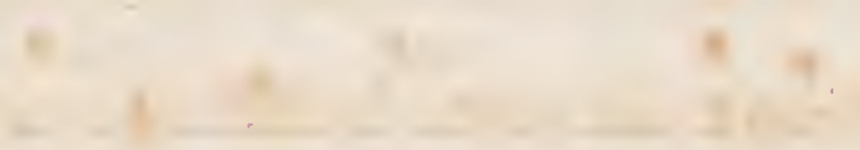


Amico Lettore

Niderarei, che questo mie righe fopevo viciucte como confra: Segni' in animo sincero, ted ofsequiofo uevso il Mondo, non gial mai come Legi al Sotto frescritio, che non no. ha Gogno; ne purepervimproceeri alfi gnovante? cher nou to wote. gini.

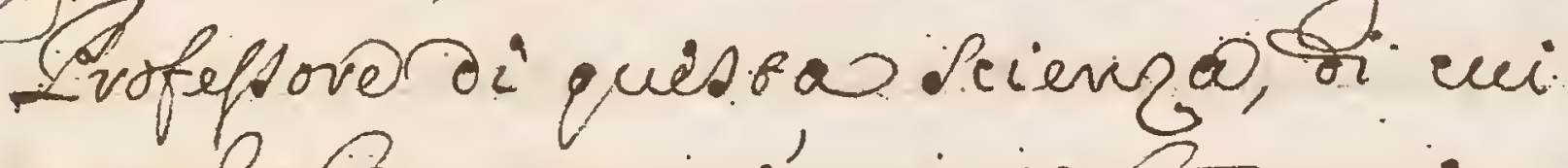

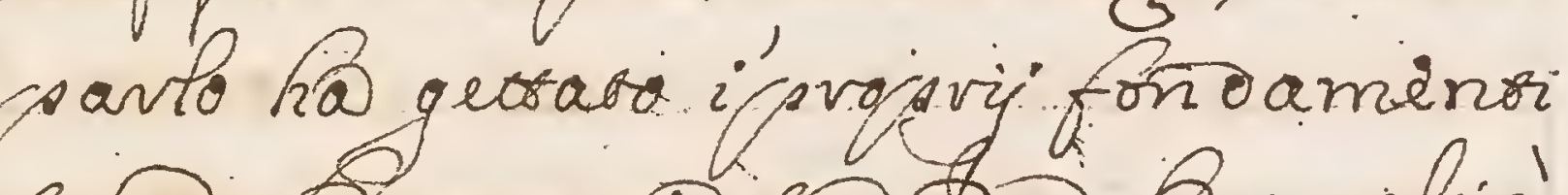

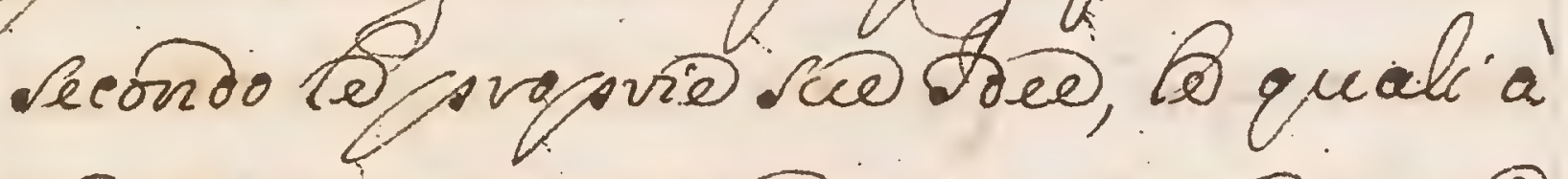
me non apjartiene afprouare, come alredt' il corrigerte, quando ancho fof Sero concersiro con evrove, o eredeveblefi

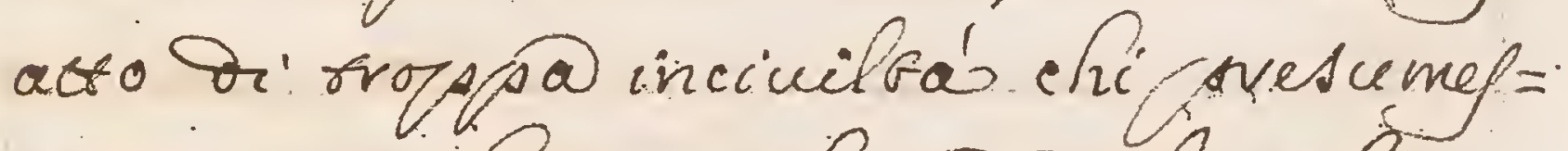
se forriotomascherardal uolfo riquei, che ambifeono it ritolo apporo= 
priato guistamenbe a' "Netruyij che sen = zaOiques@oruervefbero in qualche pars El il uevo vionatio hicen Marigite

D eon parrialibàónclinazio. no Soisfei al mio obligo, I quate fre

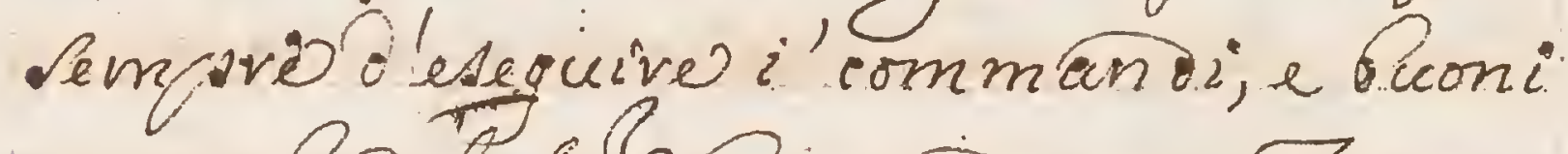

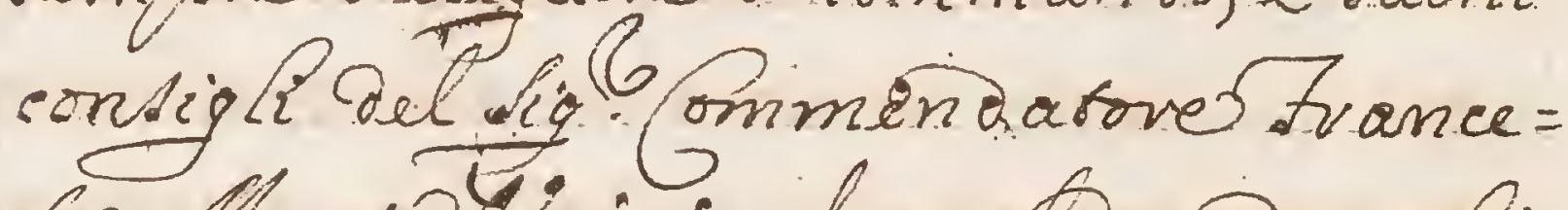
Cro Mania Taini, al quato ron ambi= ziono aniuo consagraso of Simpation del mio genio, fonto rabo uno bes. - ú carriali el affectuodi Protecsori che augsi,2Gualiever'infinióm in relligenzo, in carticotarenellama= feria, Di eni fratte Laubovita'del qua

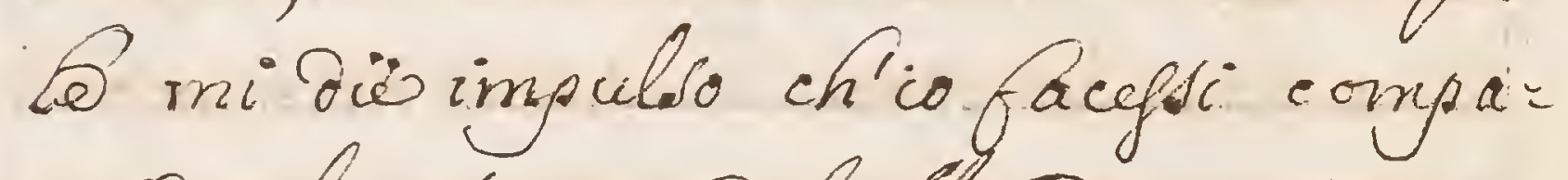
vive nel colpecto del Moño cunama:

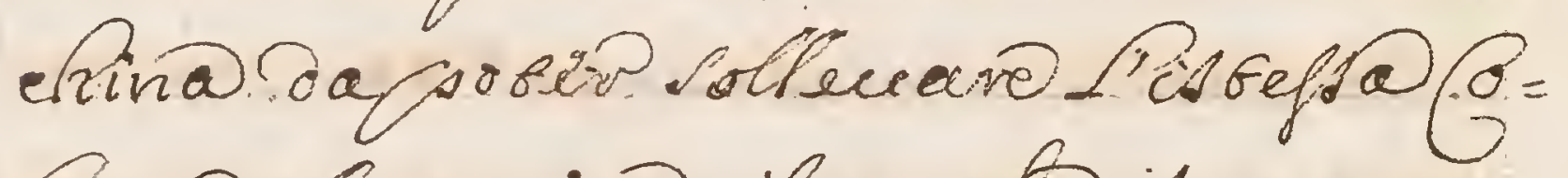
onn@onfonina, il quatocigno ule 
Sodiuo nellosurv dellamia. vegre = ber2@, et il medesino aualieverovia ehioponjfi donna in carbanefe: eo serfuordifeto olleuare un Mo: Jillo, il quate serferramente covrit: cosealla nia inoinbione, olo fece.

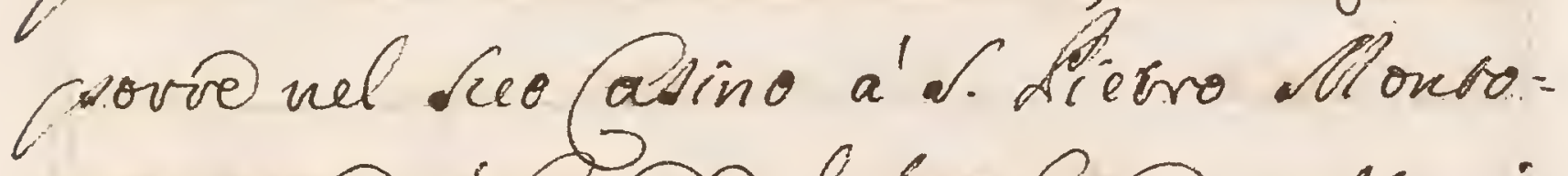

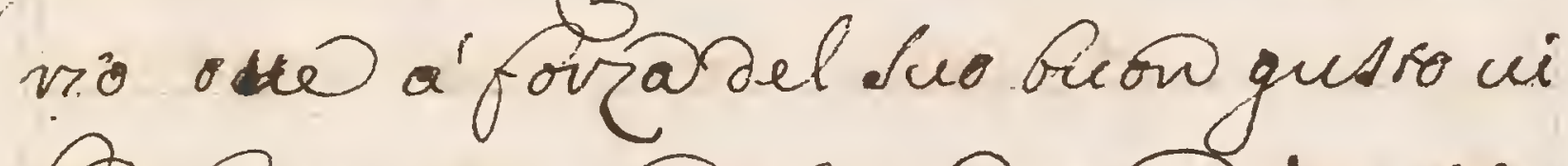
hafatto ficicañ.enj@pontivatio.

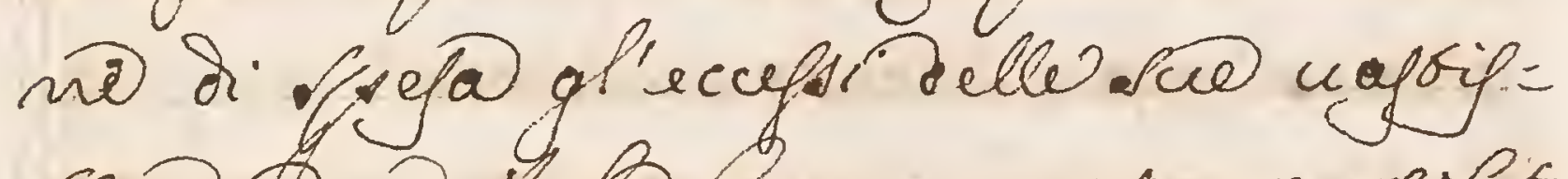
rime Wew, che ho prouvaro portifor. to Pechio nel mo. difegno ai coresso ci= Fro. Paun ancho conviene the finfel= Si: o' Letore che if med limo m'ina ufa. adesporro soto Tocchio quiste mal'or= cinate felfate, nellequali non froceavai

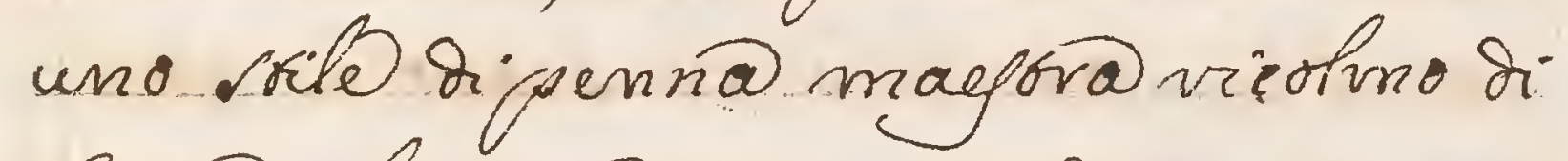
frovia eleganjaguarnito d.jpirigutio 
encedisioni fregiabe I'abbigliam?' vètovi: ce conjoito con if ale de viuarifsime ar = gubie, Gggiadria sisarote, Gizzarianel a frabe, millo aln belletr; cho (a)

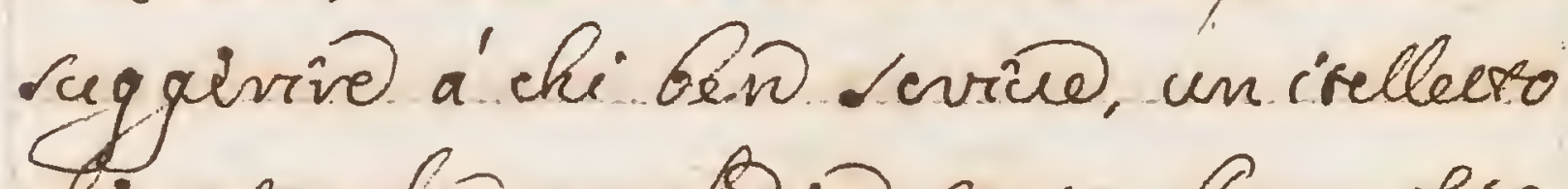

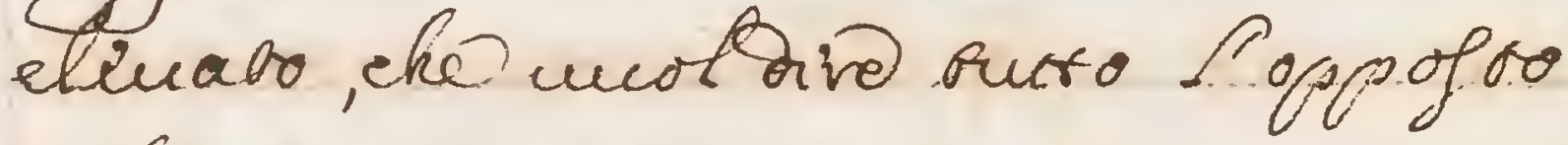
oel mio

Guerálaforimanolia,ch Amia/cenna.corro, cridume sivag= givardi fra if allondo evrivito dis bifle 6aja vacfo Linfimo ars, fífrego lexore conf worequenta cho procum sol ruarta,-cifend infa, acción on riman:

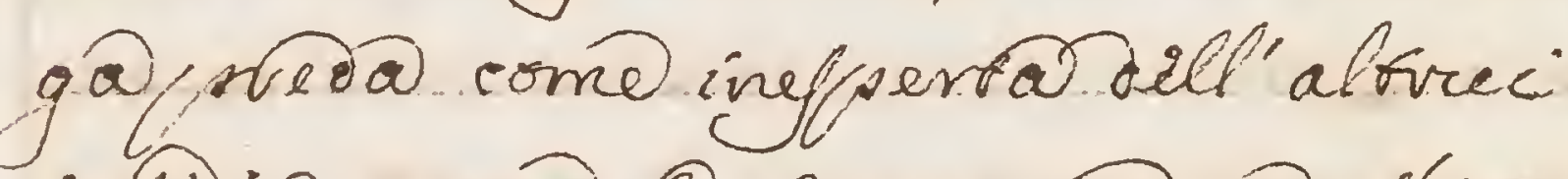
inficie, opune. Sropero avdiba. Stinal vafse contentati o. SJtenenta, a ccio now noui बiaured un lcavo, o come quesro non covrefse vifchio difimanen 
catrigato da quel froco, ono suretede accostansi.

8. Pecer mai mi güngefo all'aicro, now giafgmio meviro, maper aciidente, che or arufsero ojeffatto que =

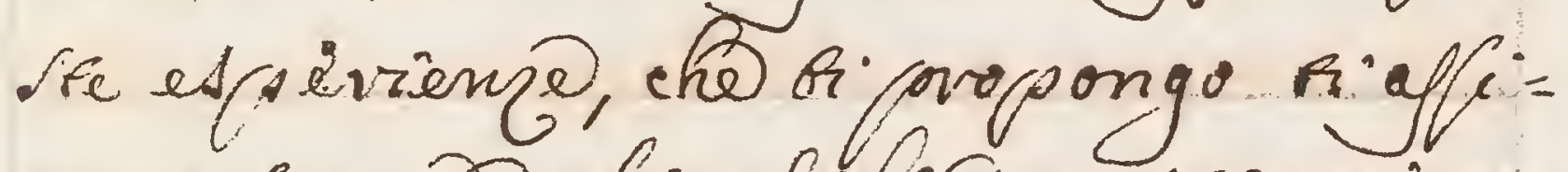

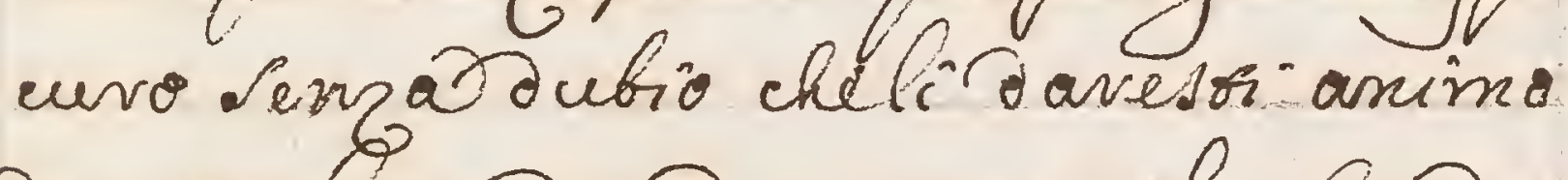
Divigigliano un nuouo uolo forfecon maggion forruna sella porefse usur = ave calla foma qualche applaus. If fillo figuepari quella sorb cho al

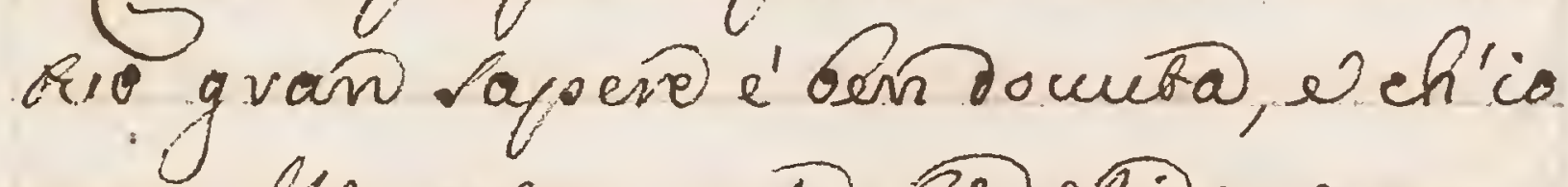

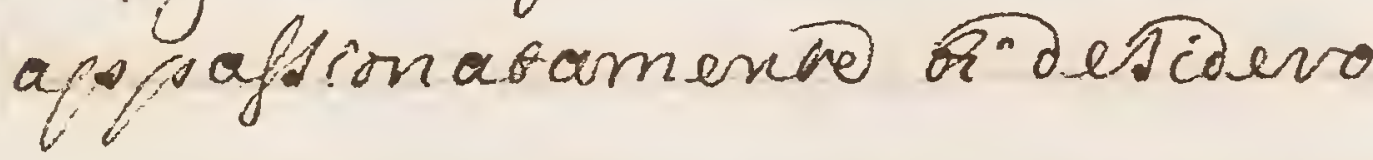






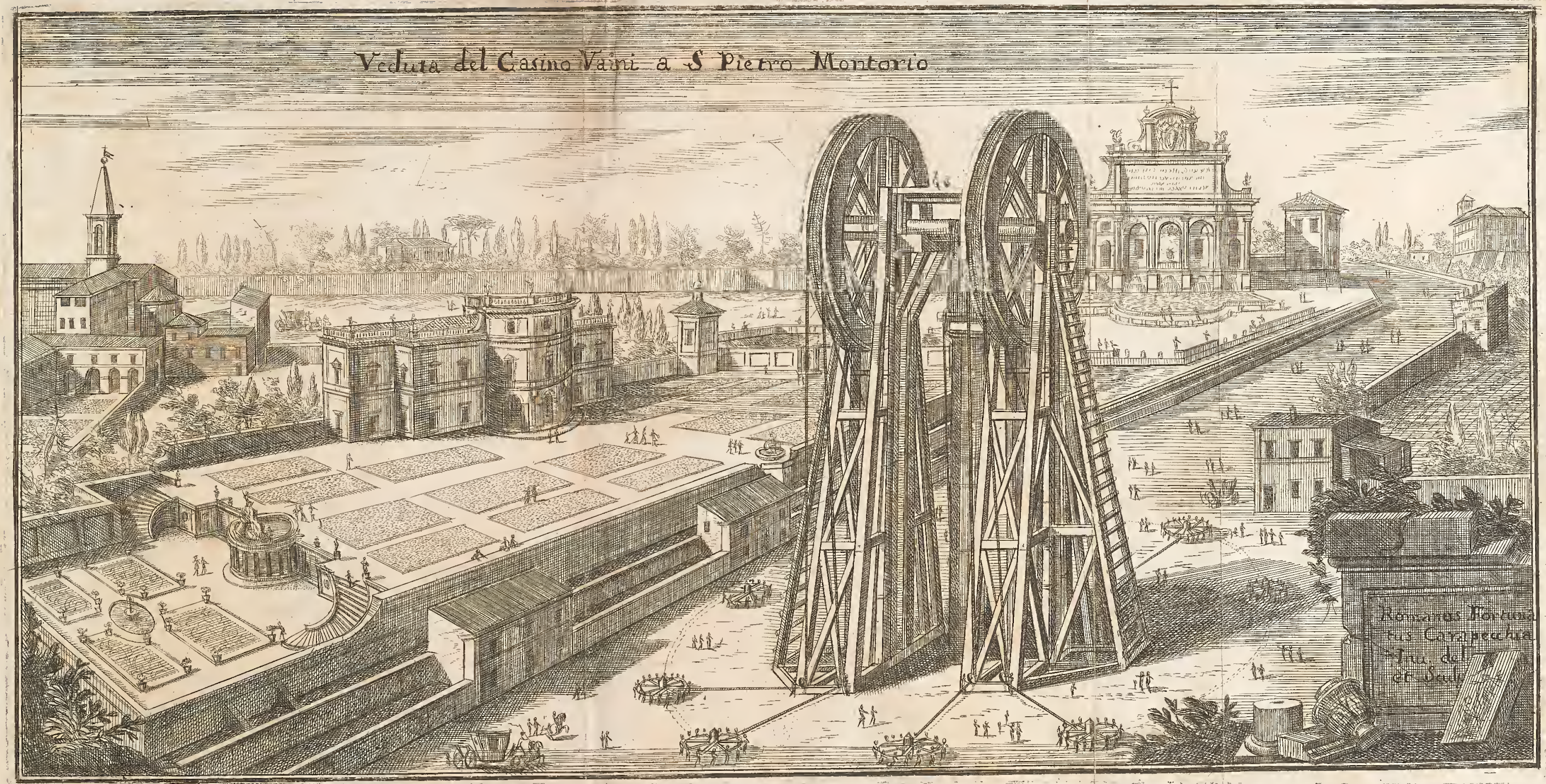




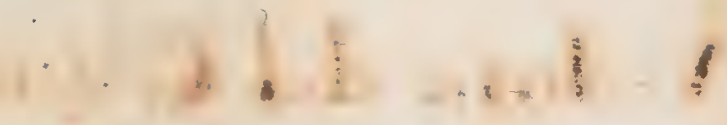


1

Tr ra Le âtro anticho mevauighes cho in quest'APma itrási-Ro. mo à nosfri ocehi sraucanots maluagisa do Barbavi sto terra na= cosie una evaquelta della/ofonna.Anso. nina, di cui dopgo ranti secoli sen'eva qua: icrevura@memovia, o renchol nel am: co Marbio alto fator ael. Monfe iforio nel. rito ápunfo dow piamento esereitano ishy= sionary in anquest cits siscovifs la cimat uafor fonna, nulla di meno non maiques@ fu eveduraper fatnso: nina, dutric andoti che forse potefse efsere. un fronco della is arovia, derumendo ral:

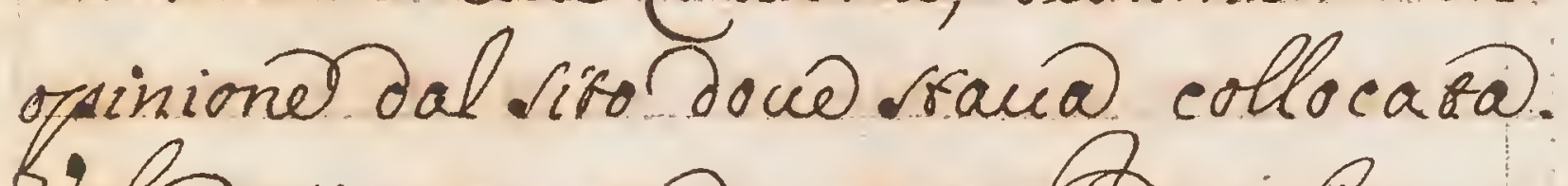
Volendosi in sanso da qui Sarvi I anno. 2703 ingrandive sfabria Del oncento furono forgati cauaro d'inforno a quella 
per adempive à í fovo disegni, má quan. pofiu' I abafouano nel cauare, panto puí seorgeuano frofóndarsi A Mloto. Aredero

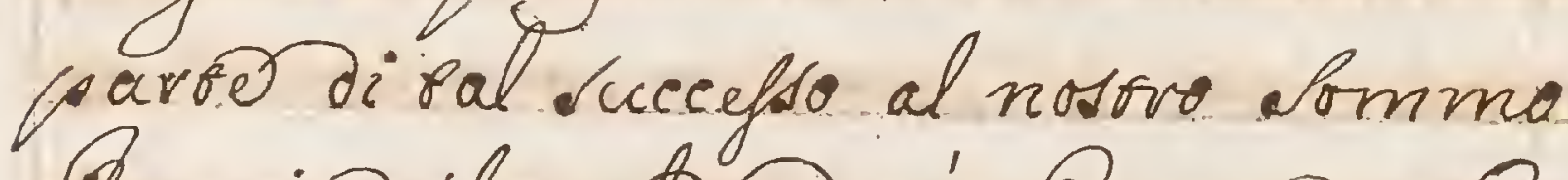
Irencipe, il quate ordinó choposto da ban Da ogn afro Lucovo, li artendefso solo àri= Arouave Pintievo sestemaibal Machina il che nel permind di cochi giomi adem =

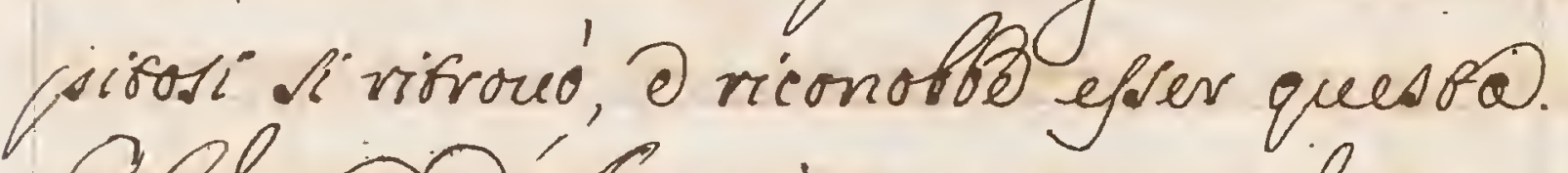
Lafonna Anronin cosara.ul suo pie:

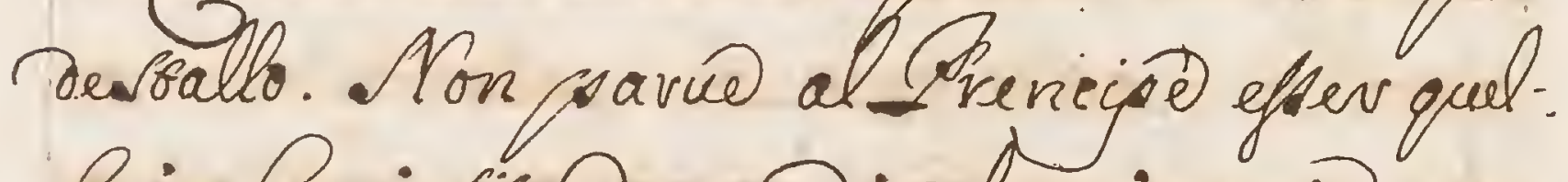
fo in hoggi cito degno di fal memoria, on = de ovinato al auclier Iraneffer Fonfañ Avchisetso, che prouapse il modo, con cui ef Prar'i porefse da quel fondo, diede quetsi immetiatam tesvineisio all opero, e topó qualcho sempo serminatone "ovdegno di= vo' cos') perv maligno accidentê non heb 
2

Beffetro il pentievo. Veivono ad un orat = Po in lampo quanriós a' Ingegnevi, ef fa oficañone ciascuna à uco piaceve pri: ucramenterdell bodegne in modello cer cauano (cosi'mi bo a' eredeve) con procu=

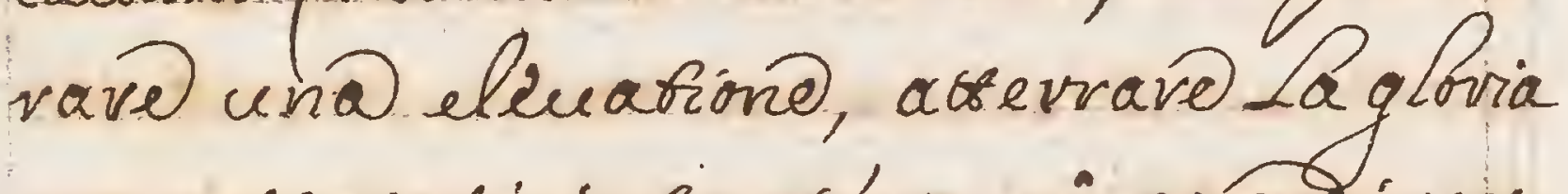
acquistaradi intant' anni con diver = - Gell'opero dal nominaro Qualiero. Lo pevo Lonsano datali maluaggi pen Sievi non cair abro mi cosi a fafri cavne un plooello, che pev bedive ás

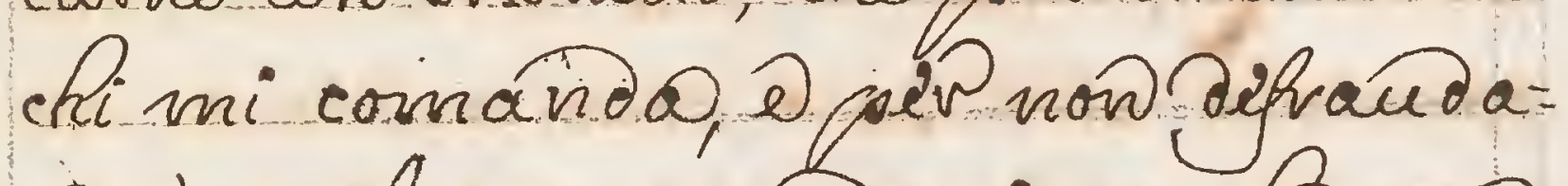
vo à quil poro intentimento, cho con pante faricho nel cortb Di mia nita ho acquifato. Míforzaro' anque in

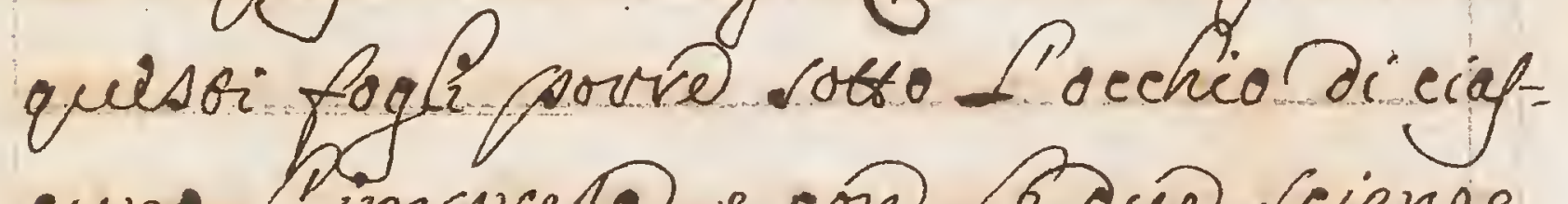
euno_cmpvesa, e con Lbw. siemp necefravie pir ral effetto, cios à Dive 
conDSeombria, Avitmetica dimo.

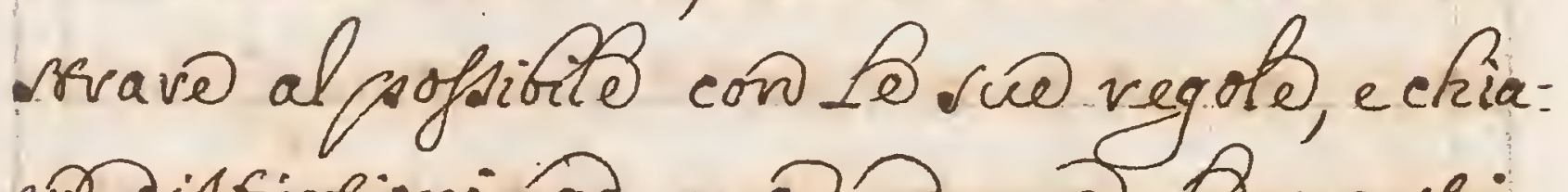
Wistinfioni ad una aduna parpi cosfifuenti pal' ordegno, sevientid alla pirima di norma gl angoli, DL A inee, S'cho pev De. SuO parri con il soccorto del= 18.ciondavivendal savepmio giu famente organizzaro col caleolavne O proportioni comparbendole, e viac=

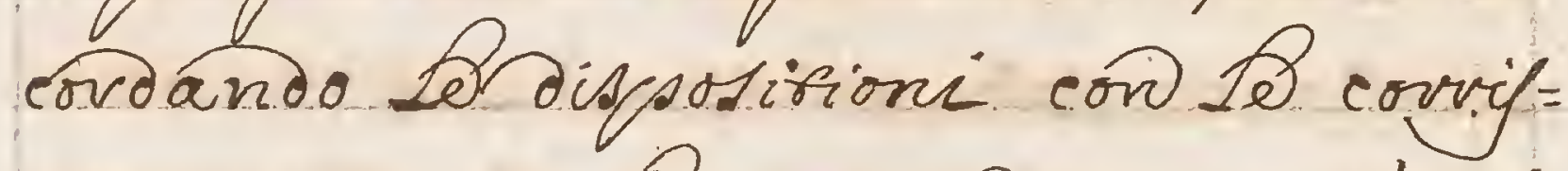
condenze, eon Le mijure, es con ípefi; come qui ah di soto daví a' dicedevé.

Más pervunire allo cogni. zione delle materio necefsavie fa misfiero, che mi cada espeñóndo in dimosrane alune parficolariba' non alieñdal fino coroposto, o pep vidur = mi à qualcho pivincipio tivo' con Ve= 
3

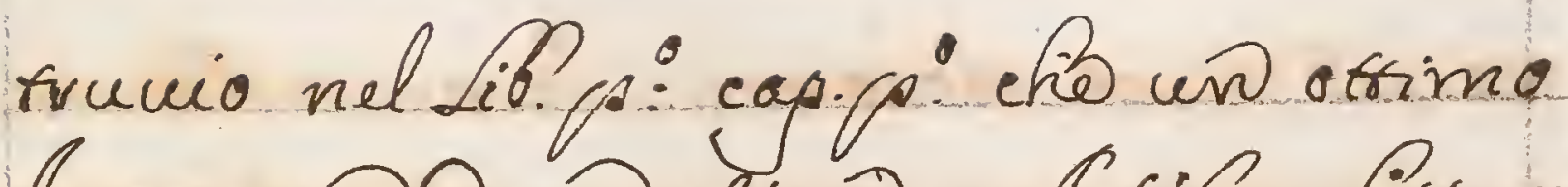
Ingegners Dewefserv = Artifex Litteva eve, fsevitut grafphidos, eveditut Térne= frig, et Opoticed non ignaves, instructeds Avitmefica, ffiforial cum pluvel nouivis Dhifsophos difigenter audicuevis, Musi= cam sicurit, Mericing non. Sit ignaves, vispons@ Luvis forsulforim nouevit, Afrologiam velique rationes cognitaf habeat = ffo uolubo povfavo invievo il Senfimentor ai quess' Afurore, acció. che à sal'uno notrombrafos strano.

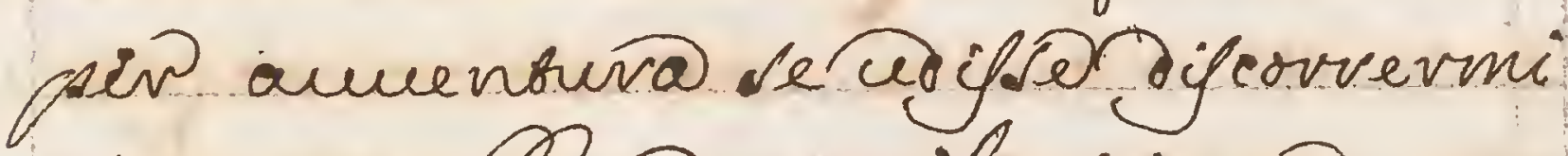
in quesso Libro di simili scienze, enon supponefse, corno sisuob dive, che io fra: mofsi $\partial$ 'entravo in méfem alienam Delle quali is mi do a credero. se non in outs, almeno di rutro qualchepicicolo 
parfeldouvo' poccarno, to ancor the non

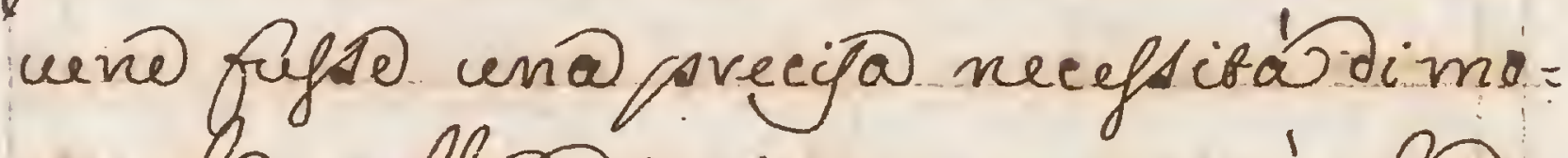
rivarto, nullar di mino avocurevio, che $i$

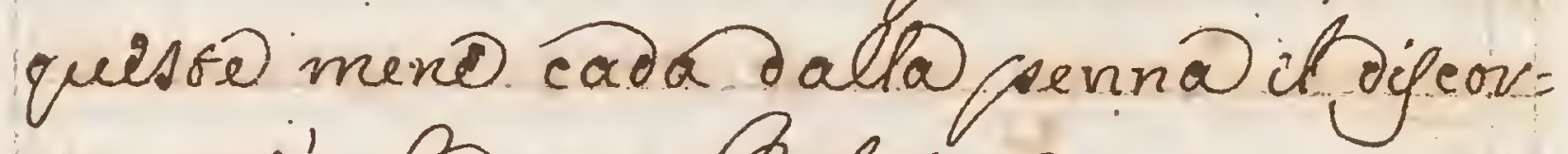
so, acció hoogniLrofefore concesica che chi scriconi simili marevie dewe in fendersene, sanrogiu', cho Vetrunio efsendo.

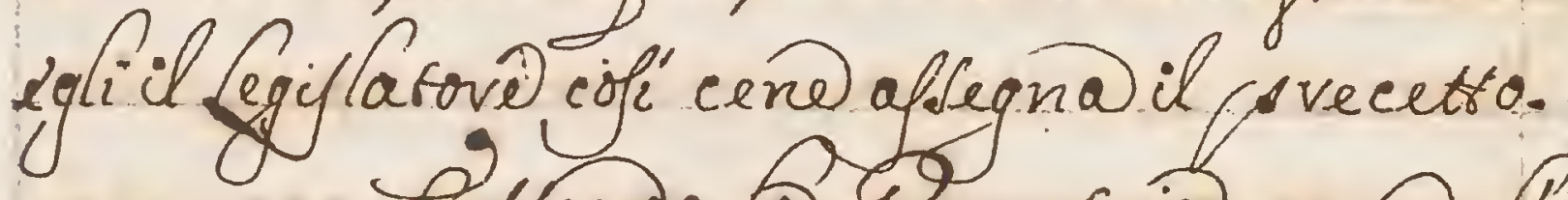
essendo abounetio, como I

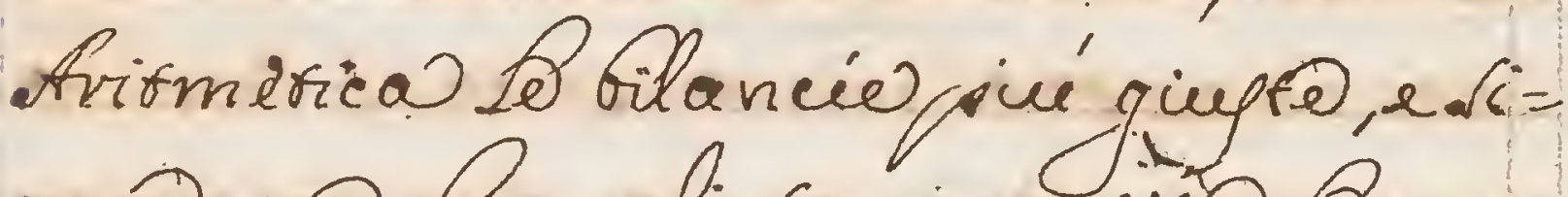
curs, con Le quali conigarijeo Ds ar ricon uguagtianze cropovtionate, che quando una now Rablia covrelazione cori I'abro pogki I"armonia, ef una

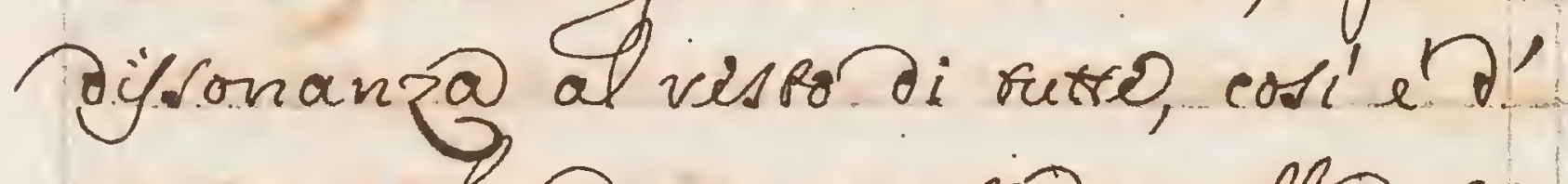
cope veghare con quejte quella. Fa: revo etho ed ouevchio confrasto d'uno sconeorde cseso generaveble crecigizis, 
Doperandodiden@ O corritgondenza

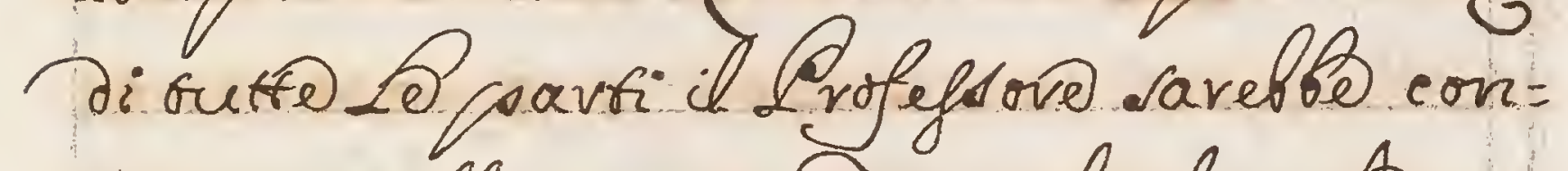
dannato alla crend i Iantalo, il quatedem = pronvimira vicino il frutto, o now giás mai no sarolla Lafami, e chi pofsedefo

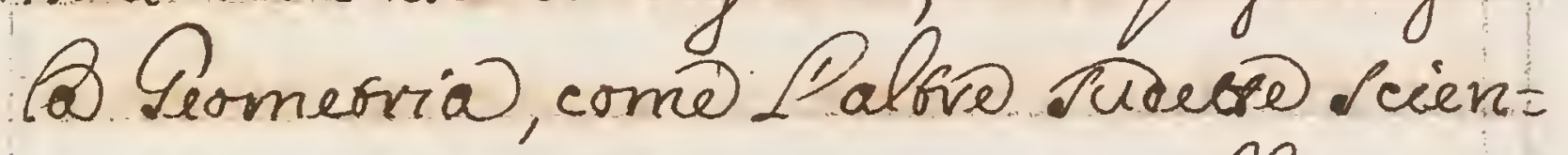
20 prico dell'afritmenica, a vebbe non atro, cho un uevo virratro di quel pate, cho pers deserivervelo acuevei necefsitás di demosfrarci una balovagaino super = Doiua,masenz@eiveonsevizionibaste= - và il nom arri Whetitide, il qualo La pen

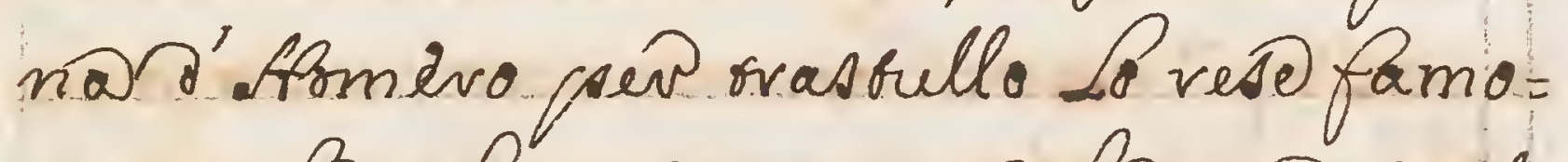
w, priche egi sefso narra, cho now sapel=

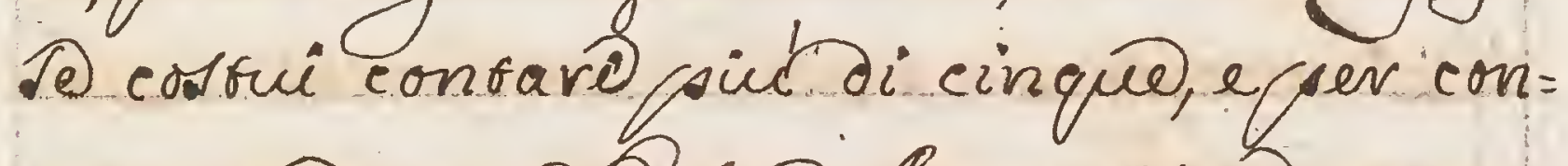
requen@Rutalosu@Avitmeticanon consifrua, chon nel numevo delle Ditasen= 2pafsaropicié olfive 
Richiamevóall@menté dido= wi'nument dà noi us ari pofsino auev au= po 'ovigind, oper darueñ qualche conrez= 20iro, cho w' on De gl 'A utori; iqualipre=

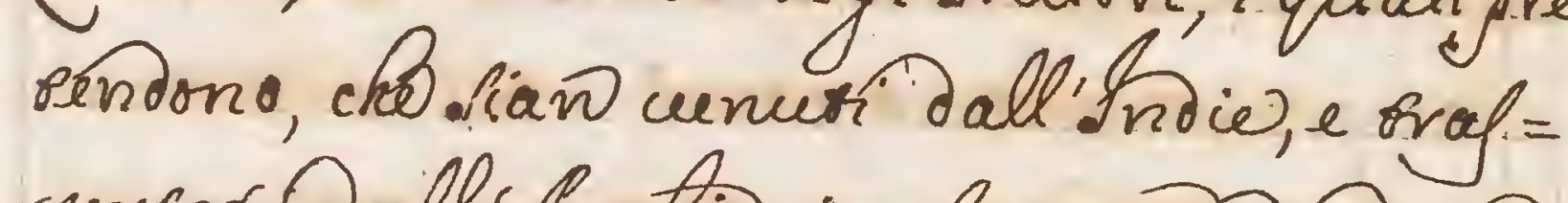
covrari dall Afratio in feagna dr owe

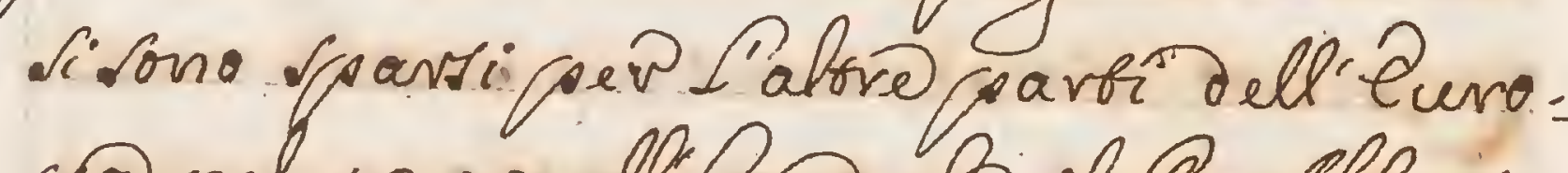
@ nel 1240 allhora cho il Re. Afforsto feco di a venive. Mattematici pers er $=$ reggens 28 Sauote di Jomeo, denomi nabesor dal we non Afondino. er = Da edaích Linumevi ono ranro necef arÿ pevil cormmercio umano ce

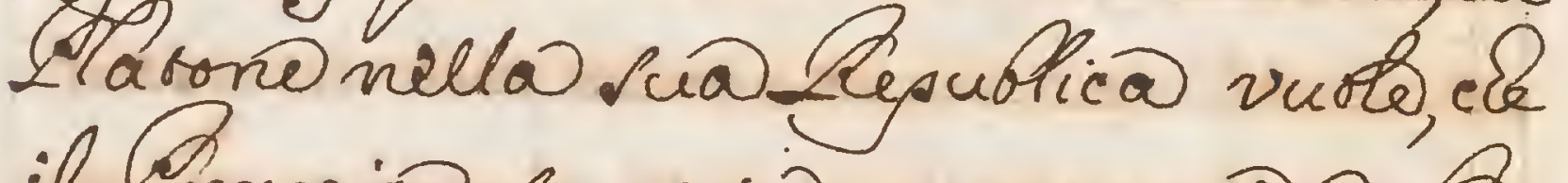
ilvencios rapia numerard, 0 a nosia Lehigionenelle. acre Rrfec'in = regnanilta.apienza all'«.chodoi. Re deLlegi, Olignor de rignovi $\frac{\text { Omnia }}{\partial \dot{y}=}$ 
5

Defposuit in numero foroeve, ef men = sura = Bepublica Romananovma Dei'gouerni Aristocratici,nat@per seggetrare il Mtondo, ser dar Lego all' Vnicuiss, atsess a'fare nell' Arifmeri: ca uno Brodi panticolare, cofi vife: rijeo oflefs anoro nei'geniali, e gli forminifor' forse quest'erudizione Orazio, dicendo

Romanipueri Longi vationiby afem Dyuint in parteg centumbeducerop Ditfagora olove il Alentio almeñoi un aysu, chopvetendecid diesig2ro dasuoi Djcepoli, vichieduia dalli medsimi il - aper numevare, da cui ne eongetur vario Di ciagcheduno di efsi nelf ope = vazioni Lingegno. fuenzorva Babib=

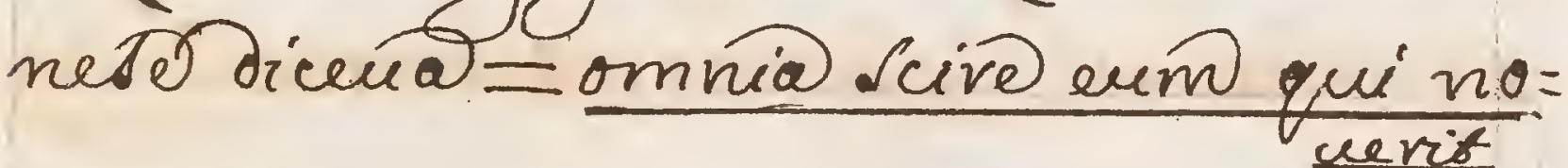




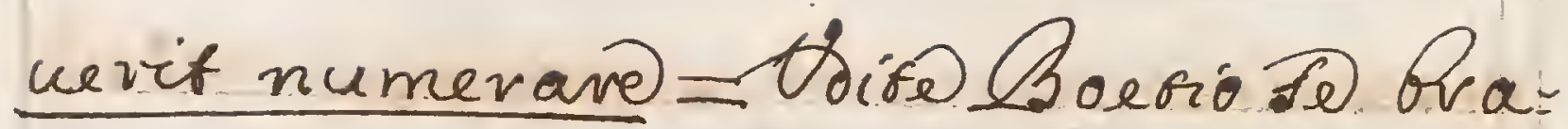
mato intendeve se con Af for a hique s@Scienzasia ovganizzato il Mondo

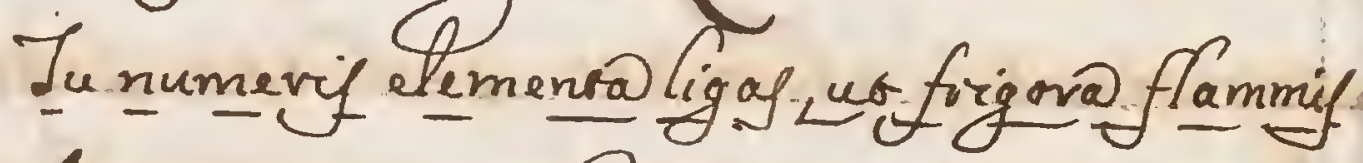

Arra conveniant ionidis

All orache nicarefifsi nelt'idea il modo J'opevarepredi altezzoriquel

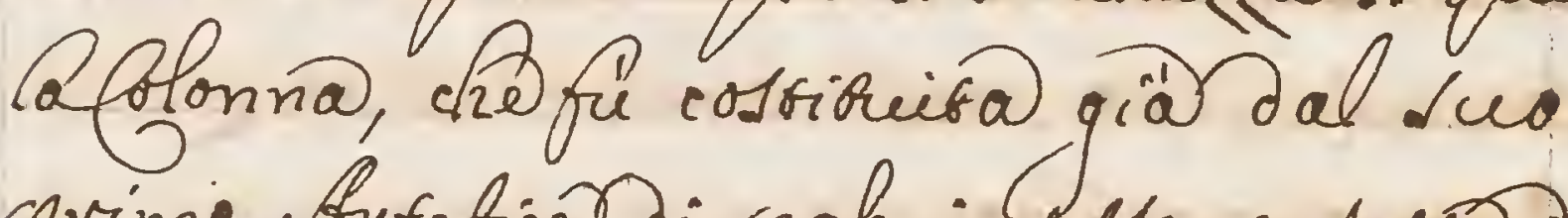
primo furfefice di palmi sefanrasetros altezza, edidiameoro nel maggiono pal = mi otso, อ cinque dodicesimi, os quale per Lapsunro è queltar ántonino sio, she in hoggidesider@ far calard, epoidévige=

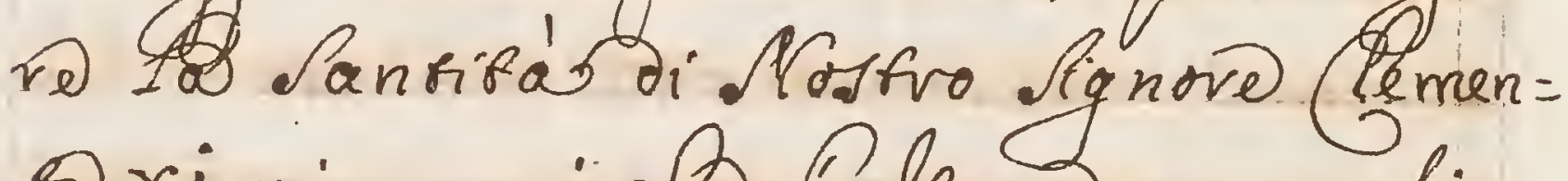
Dxi vinuenni cho Talfeza raguaglia: tamento con 8 Lrgherzo corfiniuano palmi ruti prentita ovecento undeci, che

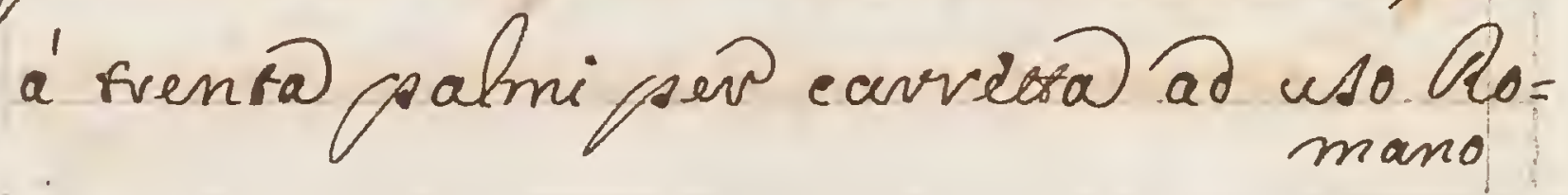


mang farno carrectare numero cerro Diece, eun berio (e como alfvi bellinge= gni no predero a cura áfarno Prese viñ riquadrandono un palmo cubo del medesimo granito ovientate, vifroua:

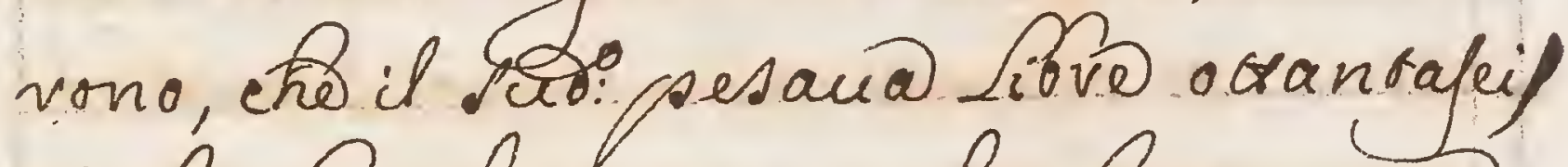
Sicho Lifsalmi 3311.molfiplicari per

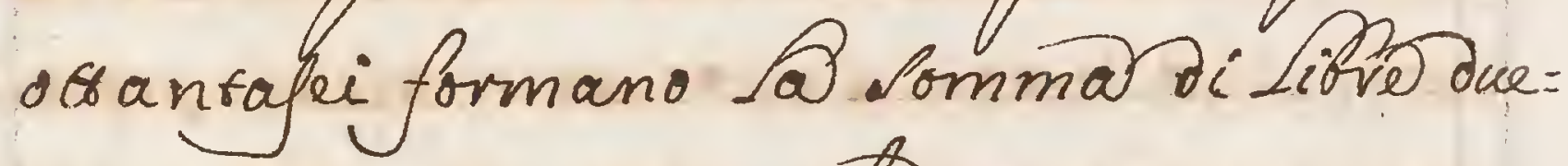
cento ofrantaquatromita setrecento qua = vantasei; to computandoui at dicicí alovelibis Rentamilo à caqione ser imbracative, Seqnami, eovo, ferri di, guavoia, ed atric, cho corels occovre= vo per afficuramento della medesi ma, D Sommatrui con Dlibe anfece DentiLe Nuetre firve oventamita, vi= prouci, cho Savebb uinuta a' petaño Q Judeta Plonna ustiolion tre= cenro 
cento quatfordici mila . Secenro qua= vantasi.

Di poi che fú rinuencubo il suo wivo fsest, cho puo divitiqualche con Pidevainono, formai in pavimo Lugo du Rofoni jiogsia armatera inevociati - forrificar dosuri contrasti ad effecto now fijciogliegsero pers qualungued parfe nel vaggivarti ogn'uno di efsi bi diamerro palmi $4>$. Di civeonfeven: 3 coalmi 14$\rangle$ come dall'ispetione del= asprefentefigura suó riconofcersi, aggi=. unrovi anch Sfigura dell'fyde de me= Jesimi di diamerio coal: $3 \frac{1}{2}$ e di cin = ghezzacpal. s2 con Tatracco nel mez=

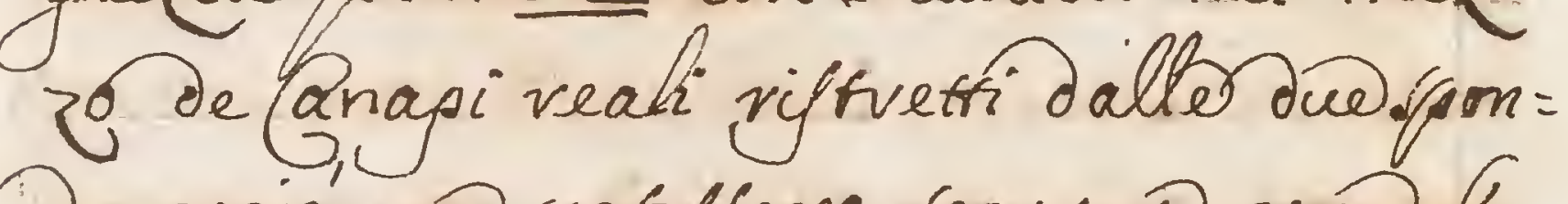
de accico non sorefsero ecourere come f' indicala Letreva A 


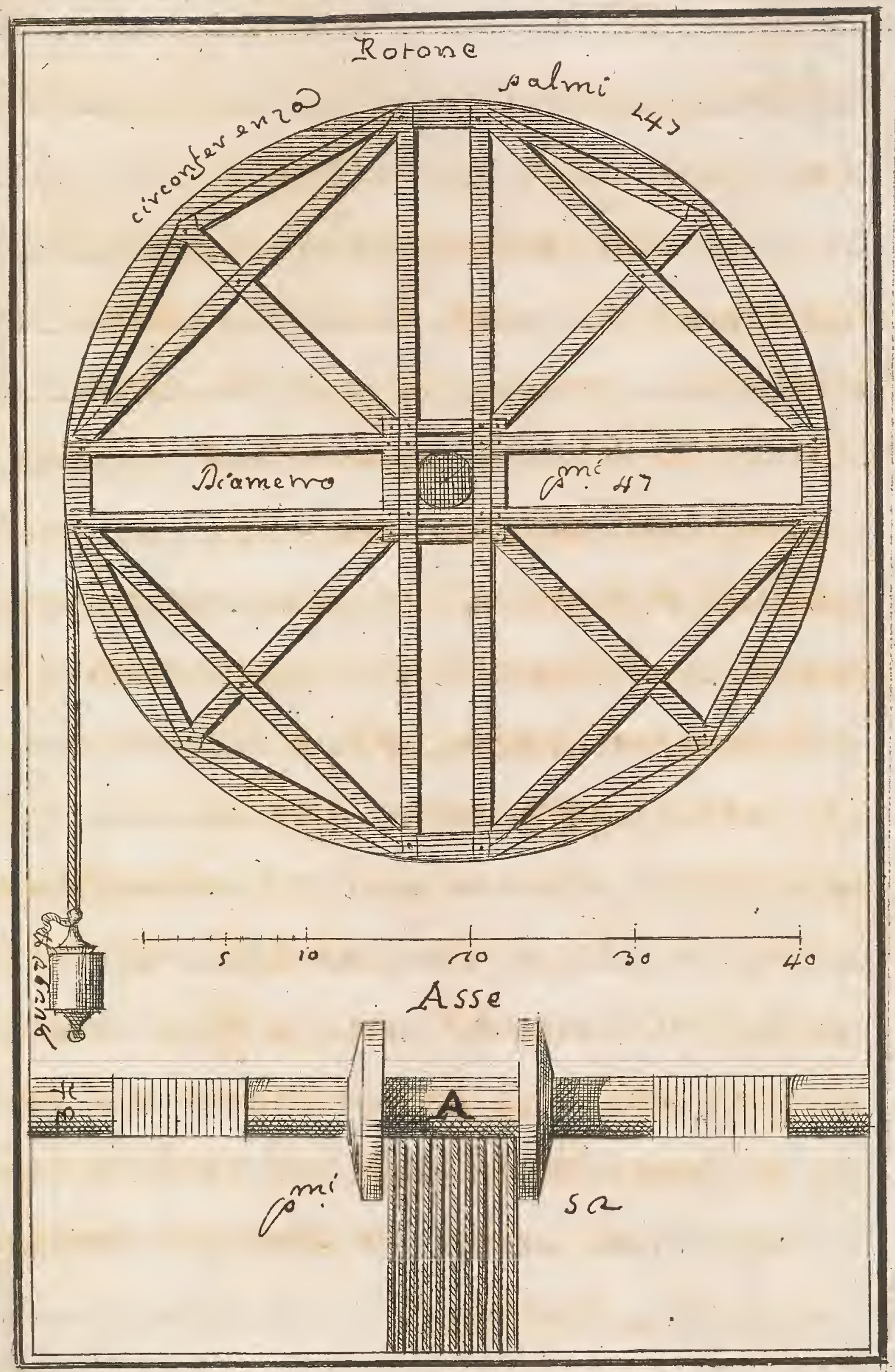



- Sicho a'mic pavero ambeduo

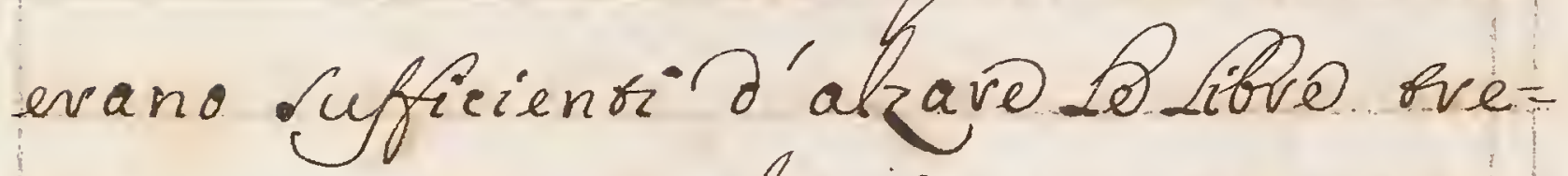
cinso quatrovoecimita. Setrecento quavan: rasii allovache. si ponefsenell'edrvem della_inia vitsoovizonfate, cughio Dive) nell' es svemitá dell emiffevo dellavora Libo unsifei mila Streenro setrant' ino in civea, o quedbo prourreblevo il mede: fimo effecto, cho opera Lequipondo quà dofoplo contraysosts al siso magqione, il qual equisons é chiamato cuolgavmen: - Lomano, del qual nome = nécio qua

Descusa Dico il Sad avo Seotsi nelta satera. Mi preuals ancho di quatro Argani Du be quali avebero frarz: upserftui sovil mio biogno, má cus = cosfir a mo nieifariy der \& raggione, che qui at or lotro lavo'per adoure 


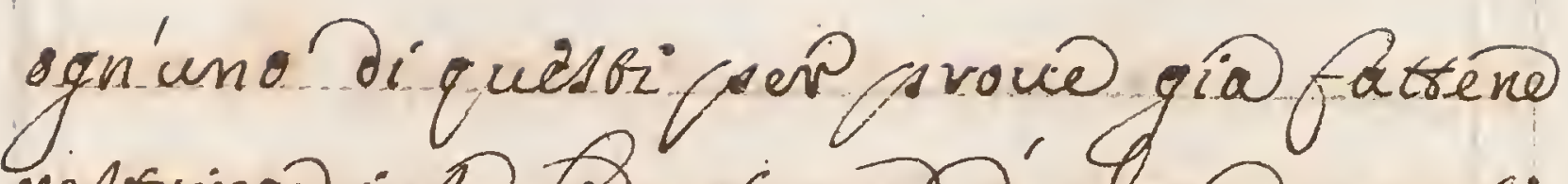
vestringe inse to porenja d'alzare uenti mila Lifre, d molfiglicaro to cunsimila Libve pen quatrio vibreuai chelom: m@menedau@otantamila;.cho

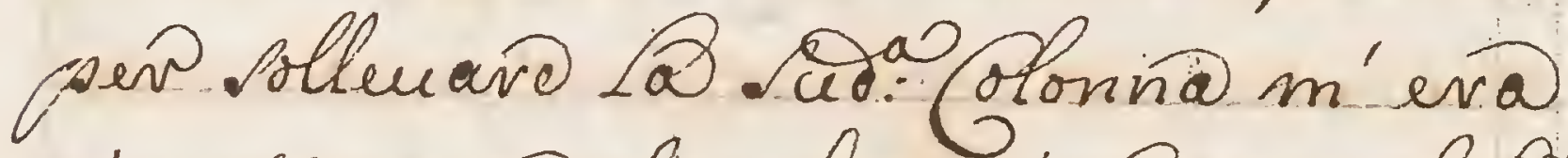
a'Aufrieienzail ualovo, óforo. olam: d'un fugano, emesz, ma come ho

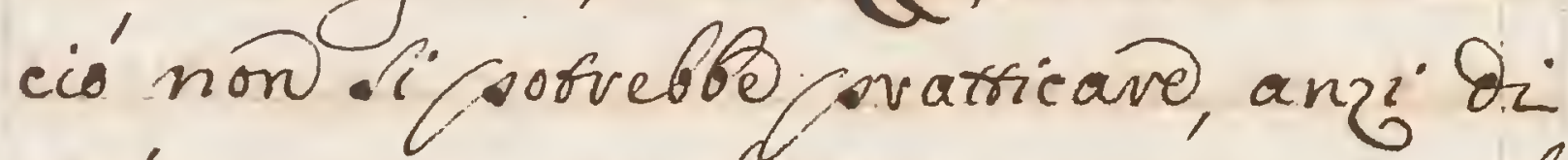
scú menéprevals' Di quarso con il moticio or' soiro quel pormenro, equilla colfione, the prouano insinsecamente gl animi de gl' Atranti nel aidere

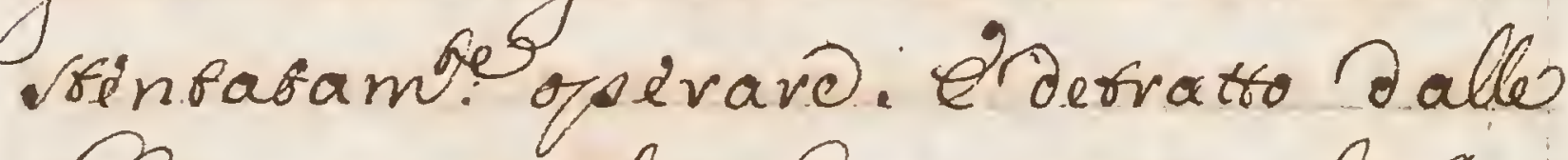
Libio otranramita, cho porbano gl friga:

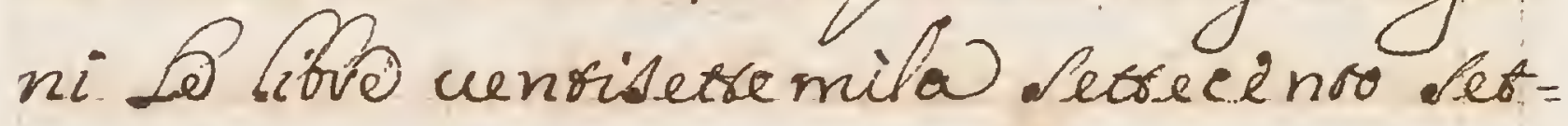
fant'uno Secondo ifldedoto, m'avanja: cuno Libo cinquanoade mitar ducen: 
9

H unvinow, S'ch afsegnando ad ogn'

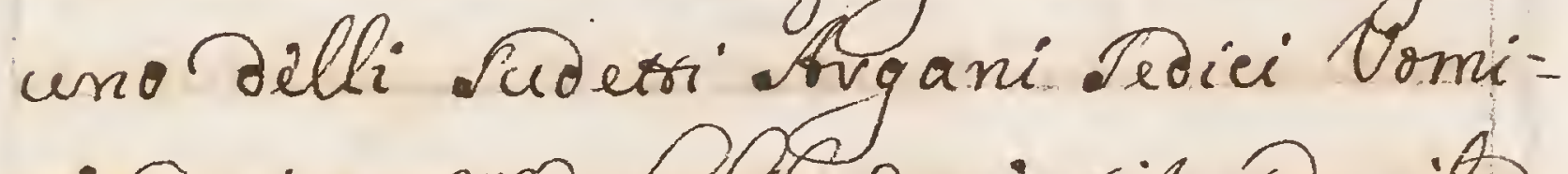

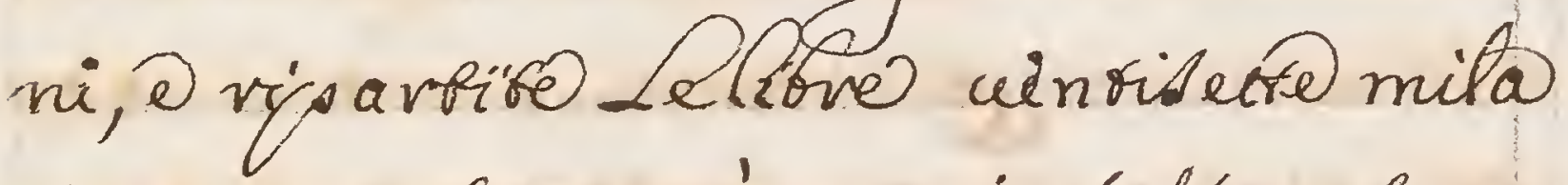
Settecento Setranticu in wefs anb a = quetiro Nomini, cho santo (Secondo mo) no vichiedono Libo Argani, vimaneuta

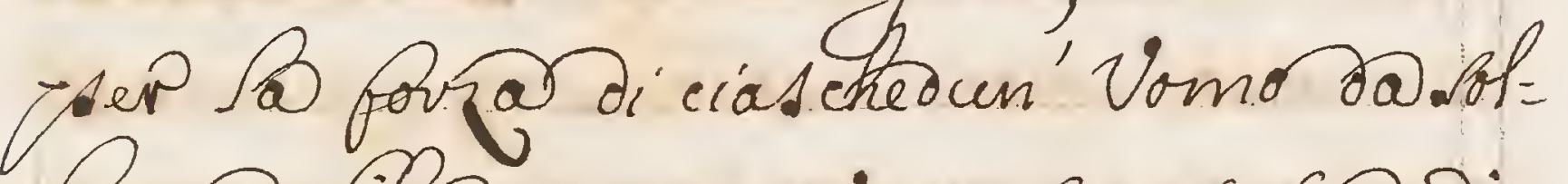
Luare Iibio quatrocinto orentabio oi ciso,maidaoseruarsi, checon ? aurivita belle sole vie for a ogn' Vomo si vendevebob impopsibilendí Sol= Cuaro Simil granitá, S savebbe igno. van@zi chi Sor infende all'opeva di.upporto, ma apsogqiati 1 Suderi vomimiallo.rangabicsalmi quinde

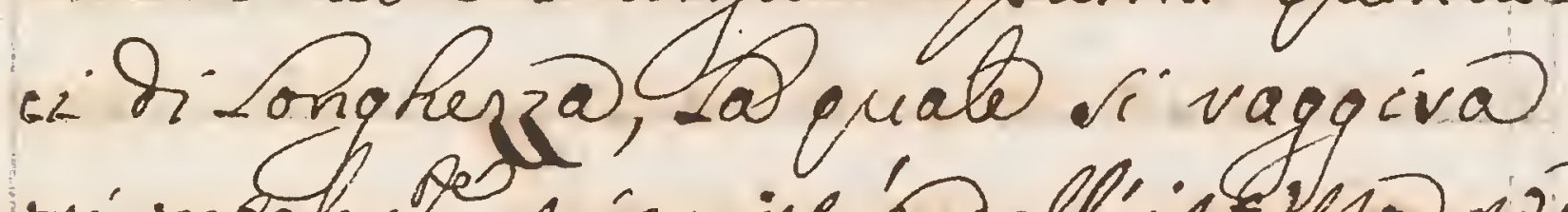
oriontalm: et in wivtín dell'istlsapiv cagiono chof D Poficio Della Leua cial: 
cheuno diefsinon sffivebse alova fa: sicha chorishluave Sibro unsiace on: cioduodmez@incivea, quiniés a com: corendevsi; chô non savebro gran pena fofse à quello ofligaro con Ango savio Dírempo, ea ogn un bi foro non faveble wolenja alpropvio ofere; Re in alora

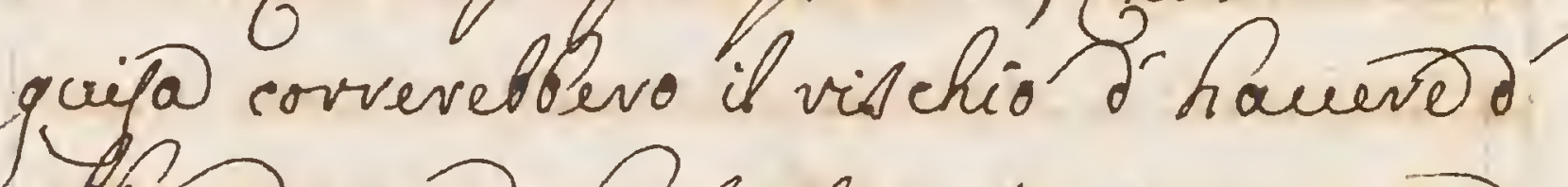
abandonare o mifuraro quacame, il quate cón pafsi dinfinita viotenga S'incaminavebo al svecipizio. onclu: Diamo Dunque che alli quatso Hrgani auovando Sefanraquatso Vomine nel= Drorna Tudeta gunngevebbe il Dro

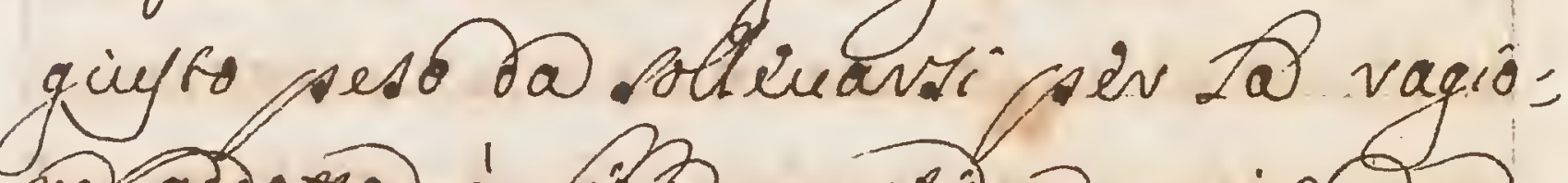

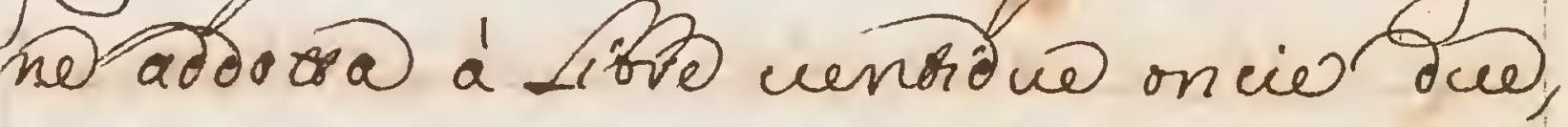
a mezzo in civea perv ciadchouno, come 
$\angle O$

Davi a' diuedere in unat dimostrazione Tramovists dunque esa= minato it Dave, es auevo vistoccai, the

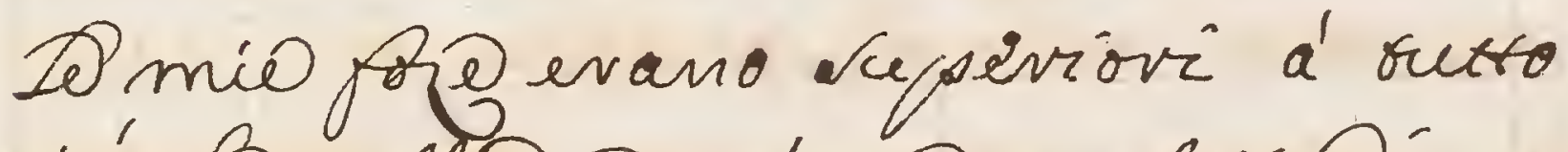
cio' che nell'idea m'eva suefifso ópe = vare, davebro raba bordagine Se non auefsi difigentimente esanninato ih Bet = so, mentro if medicamento quandono iforma croporis coiv quel mate, che - Desidera curave si conudrte in uele= no, e comedin e'insegñ. frehimide sempre deve eferci 10 midesima/so=

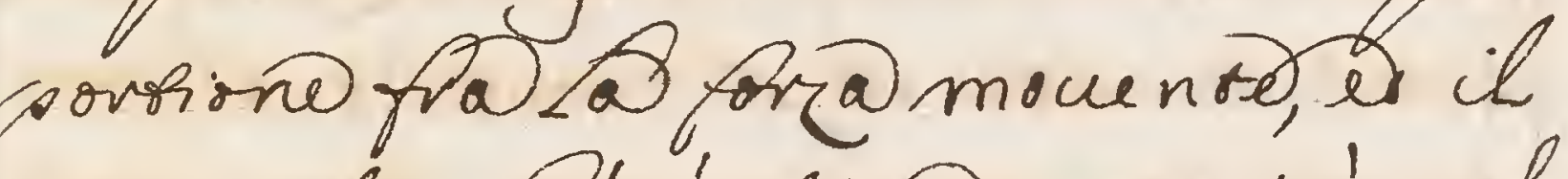

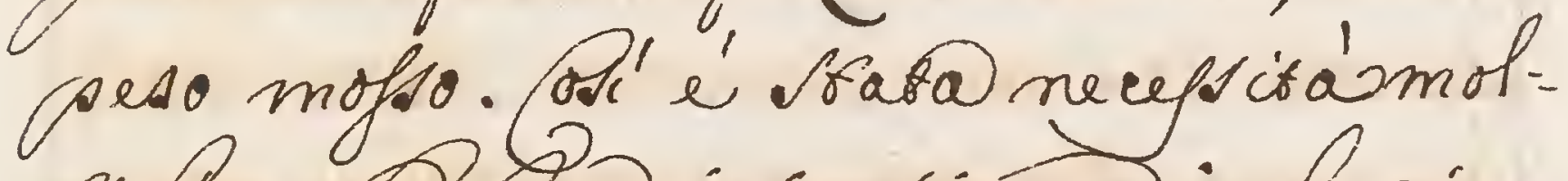
ripkicar lefore in ranrioi gradi, che gion: gefare a' costifuire un equal forten: 1a, quando questa non fogle contem civara, e che visenefs un moso griena: 
Bo sprimevebo, o noñ viceuevebbo sl= Sievo dalla porenzadiurice, o cos' Savebbo 'issesso che abondonarta all' impero del properig Scco cieso

Lirovnavi hova a' faced = Grobell'trgans, Del quate in quejo Didegno porvete condidevarne linuens: one, Os corenza del quate é cagione del:

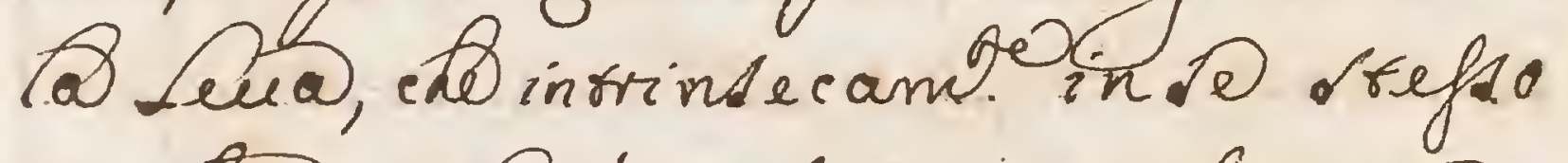
racchicede, farva' confefs avrui, the non u'écoreve chofacia avgine allor for bdella medesima, yando jevó croportionata al piso, con il quate con:

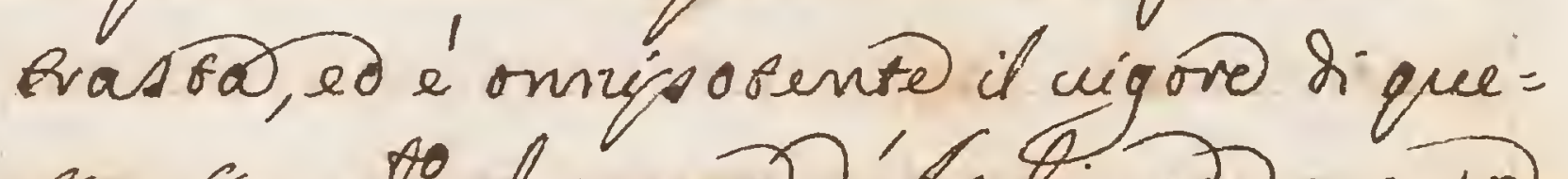
so from to al paverve 'Avehimedo, mentro eftendo To curo scarorigino dell'intelligen: Ogeomericamecanica djficilm. puo ingan = navsi, come a busti evofefori é oen no vo 


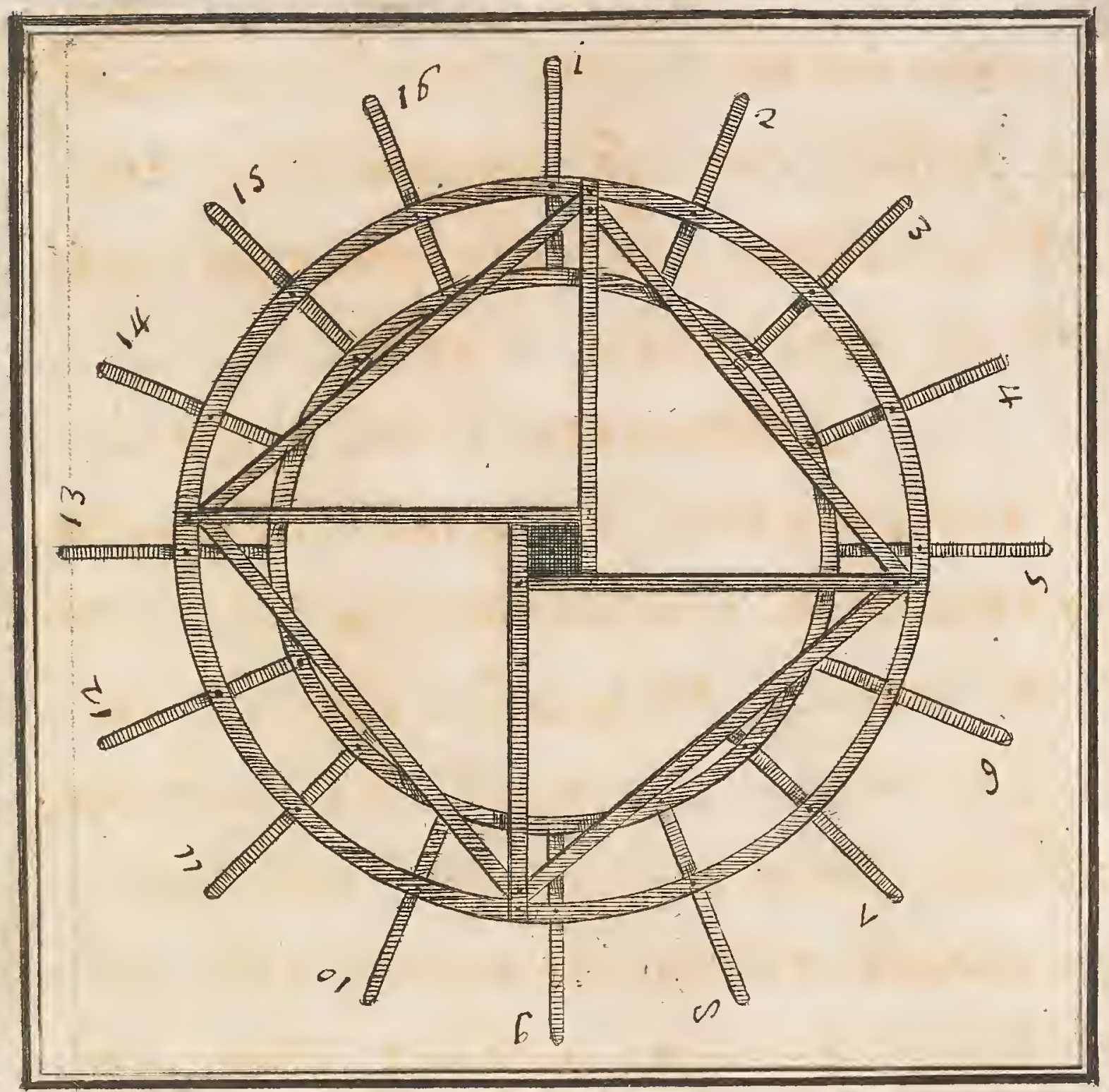

Royone dell Argario 

12

Oounque ho gpevaro $\rho=$

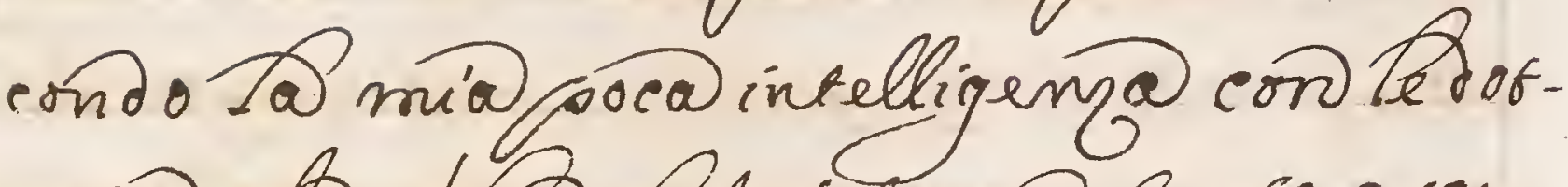
orine, che chas Asciato un fanso gran - Plastoro, il quato nella afeoroderuot biriene.cuo@aperta piv-putiqul=

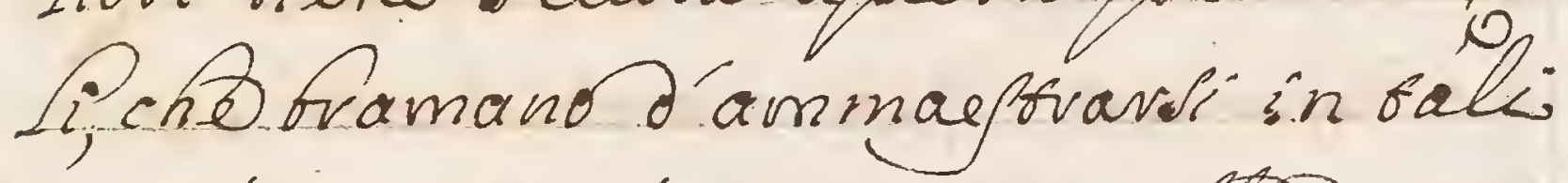

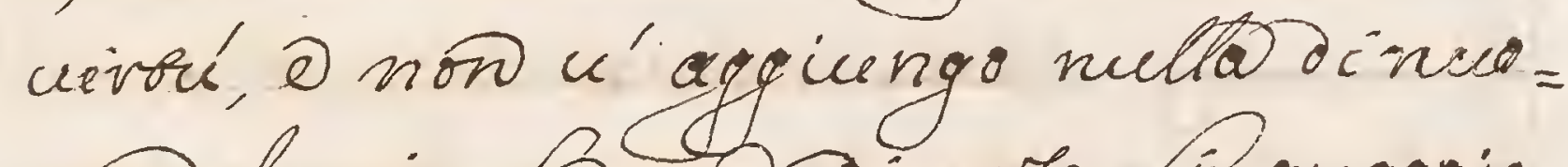
wo Del mis cho con oruerto Inquagio sal hio, on on pavra' iasanj che

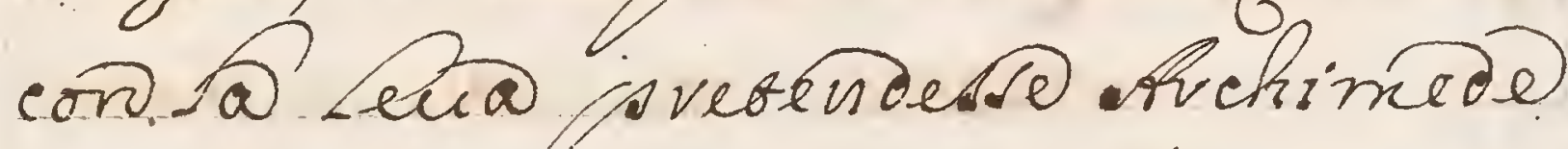
direndersiggniconapofibilo in mabe= riabi traridiceño il medesimo de quando glifofse trate sermefo h por = vill fred un salin frior del Mondo cho gli arebos anchorass Ofrimo Di:

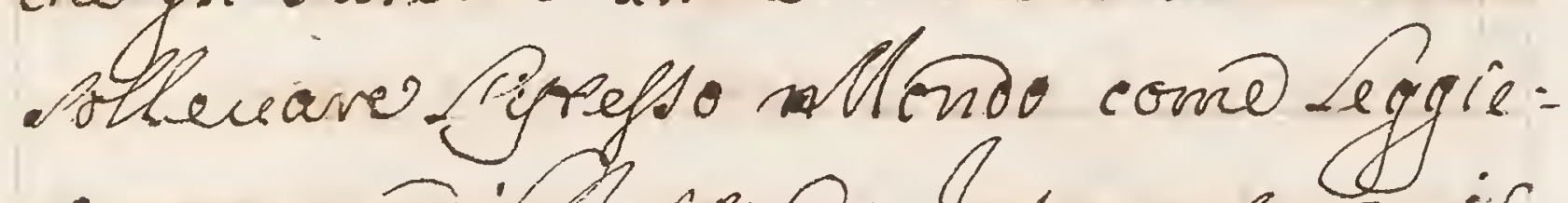

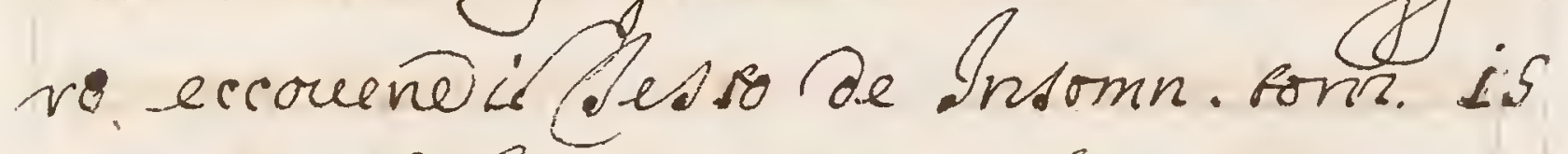
$31=$ ofverimeded ocem aliguem expia 


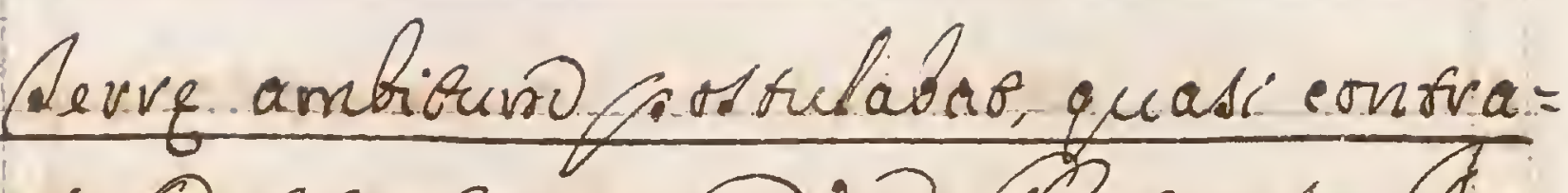

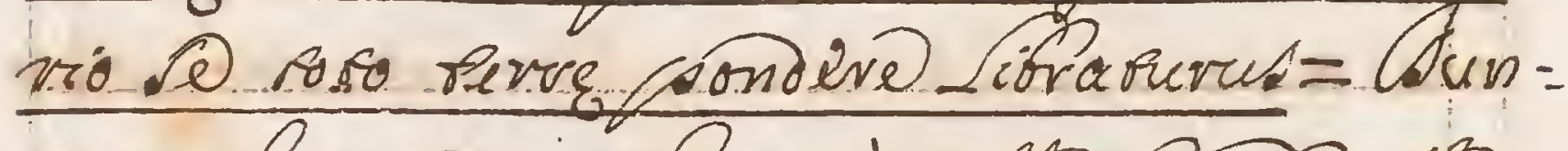
que hinon applacuiva alta forzadela

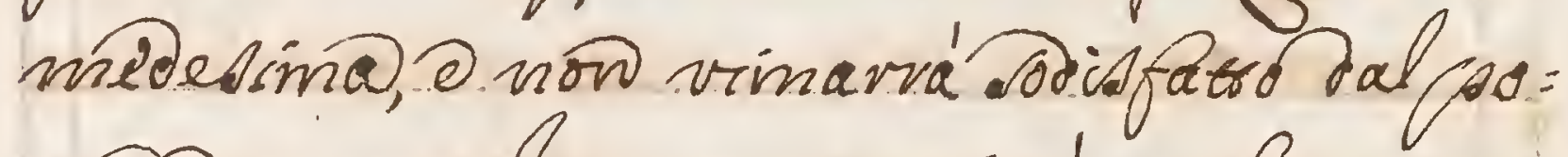
pervoi quedt Afromento; avá ancho pazzia

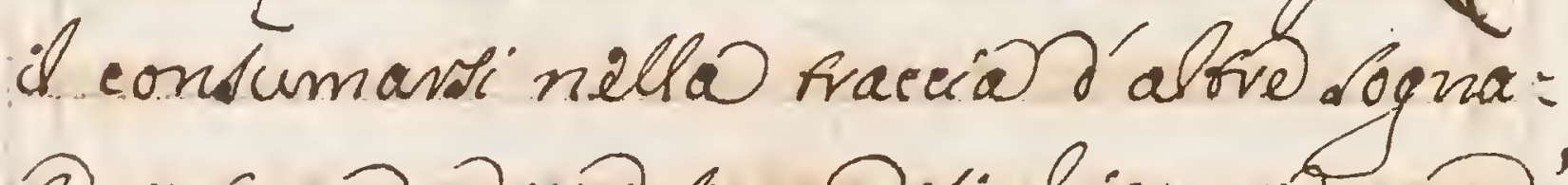
Pe poreño, anon seventosic chi operva, quand

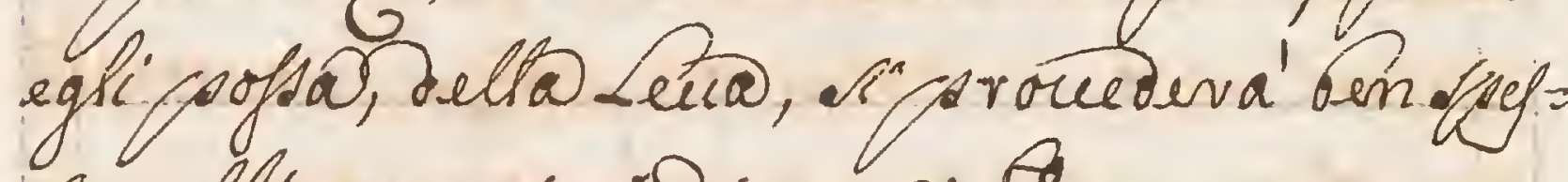
- nell'gevazionio penoin?

Afncorchogn'un intendache

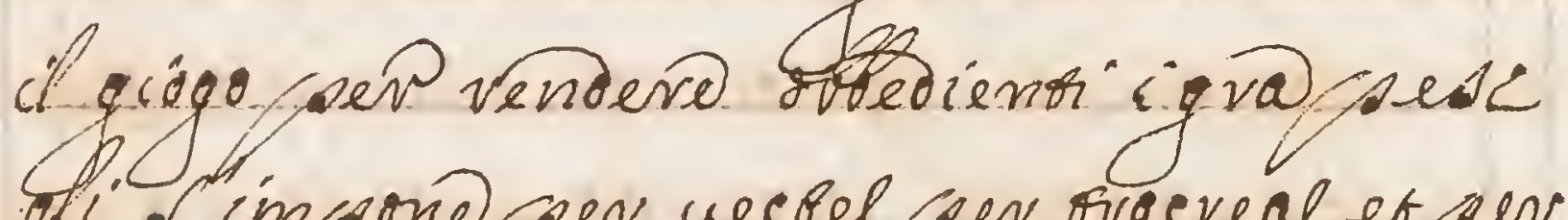
hi. cimpono per vecief cer ovorveal etpers

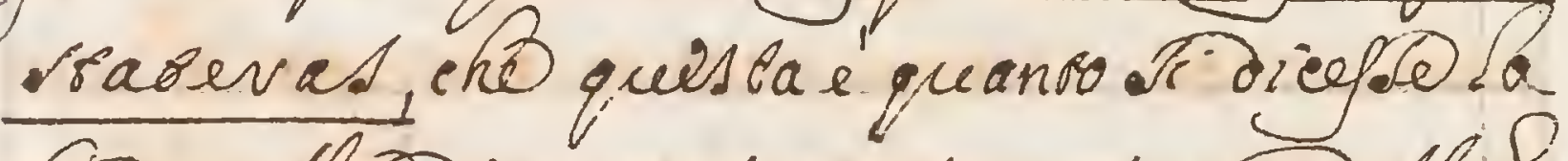

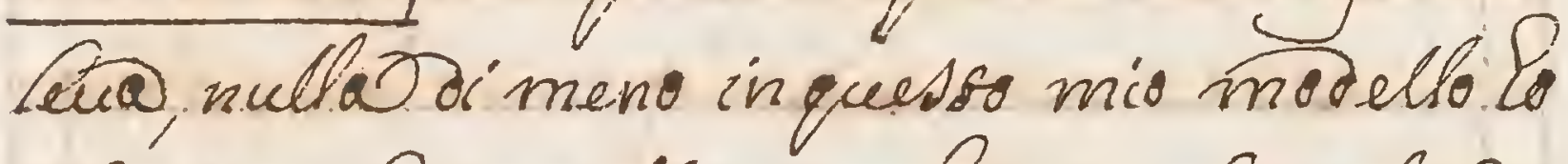

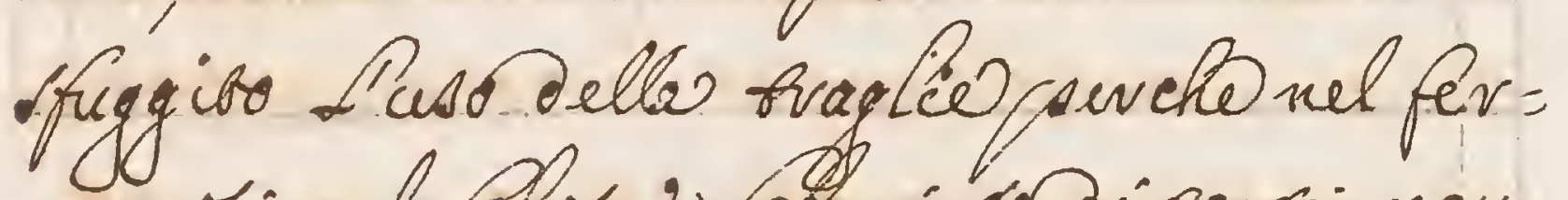
mavis nel Chos, 2 adevinos bi vansi vau: colgrimentioifuni in quel confus aggomi= som. pano meno un f'abilipara a' gevine 
13

gevirne to confutionis. If vicorvere gacero allo Avaglic, itrio choppo manieva da aisfovezzardi avelbe un achiarasti Noms

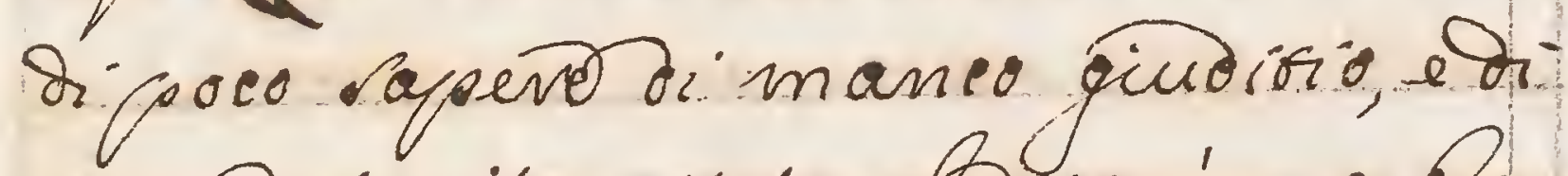
min or Seguito, attes cho ogn'uno ben intende eho tomedime con quel infi: nito ammatafsamento allegevis cono $i$ anapr da quel bormento the Ofrons men.

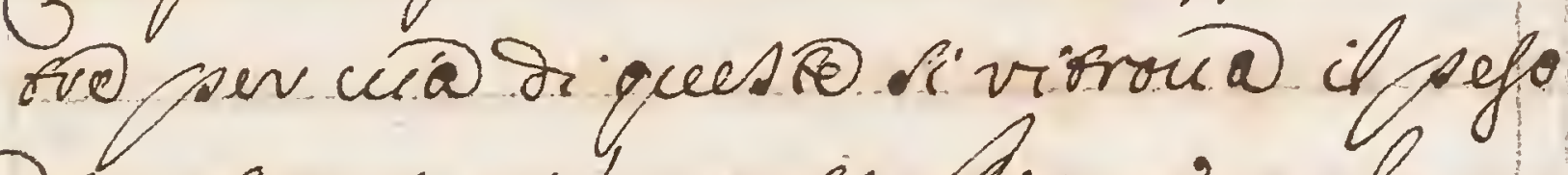
Dicuevtito insocic parki. Aico pervo ckequen

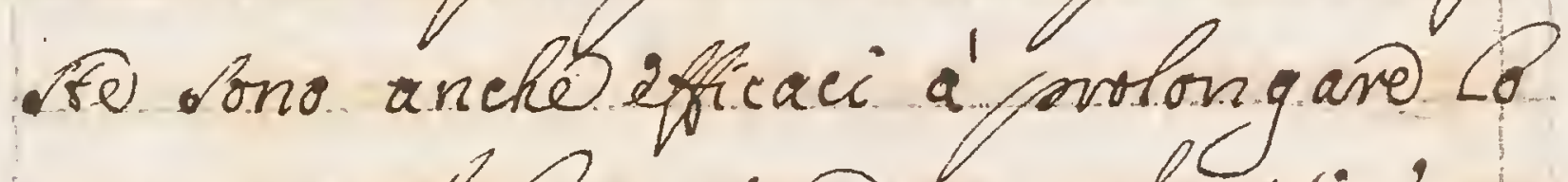

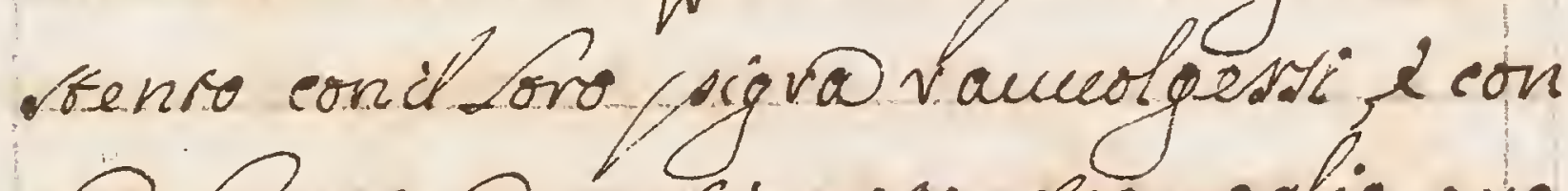
in Ingo, e consinuaro orauaglio que:

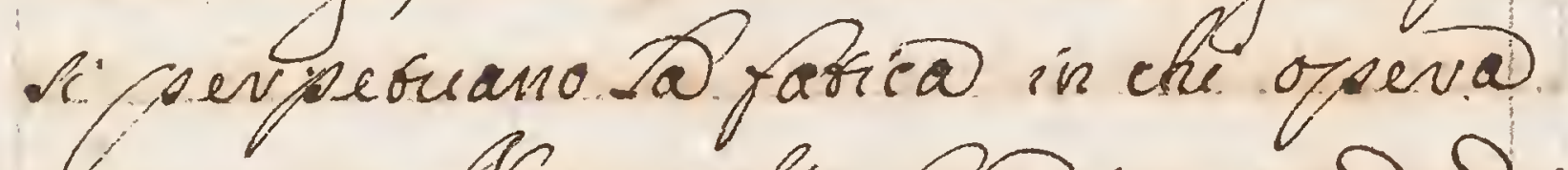

Ton colso far acimeno or oi: we, eome cur anch non wi ava'inge=

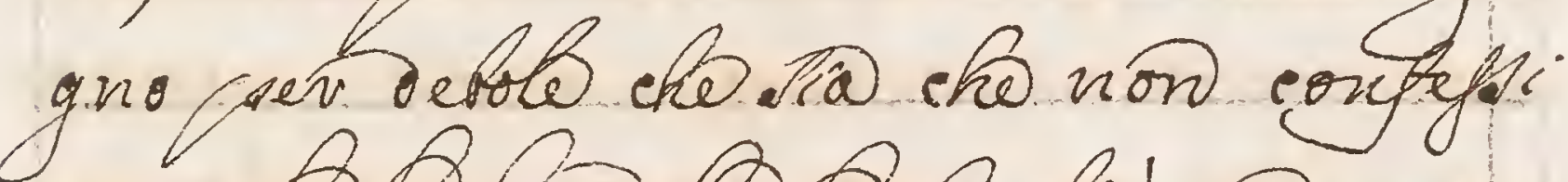

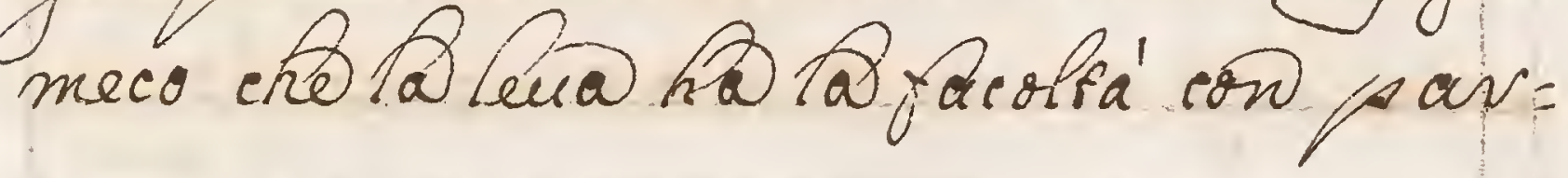


sicolar privilegio divaucicuare to maferio ancor che morpe nella depvefsione hipelan: Giarmi Alacigni comparbentoli im mo un

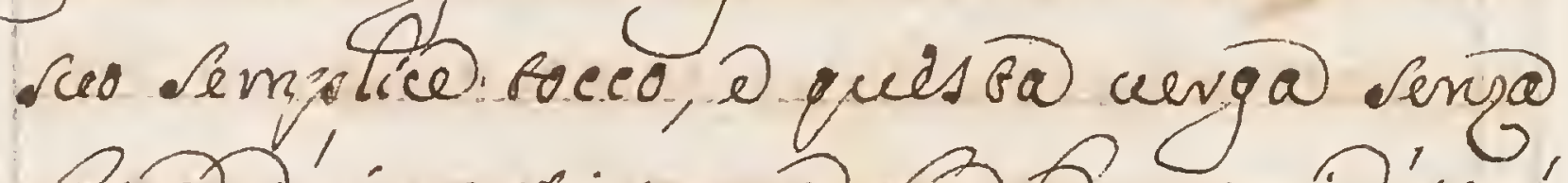

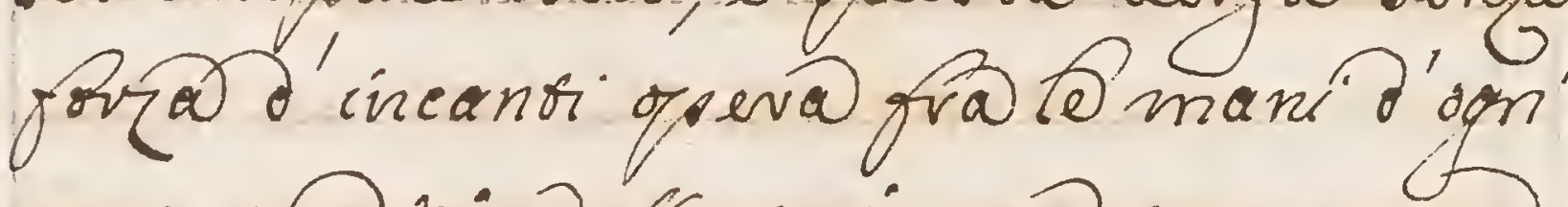
uno prodigi, D fupori, maper now ineorrentin una baceía dicoco esperto

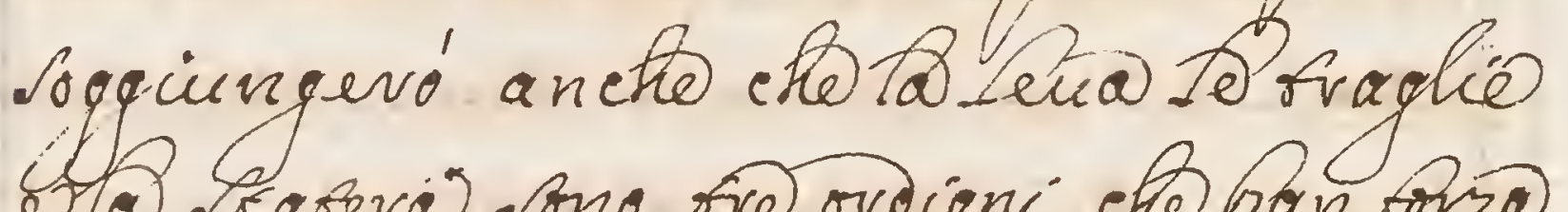
10. frabevi ono oro ovoigni che han for Di ruperañ nella Trafica, Rsi fació aive, anche Timpoffibili

ori cen motice hora. foucenuto. mi virornando altafondrella_eu@, L" - Areo apere pir qual cagione con banta

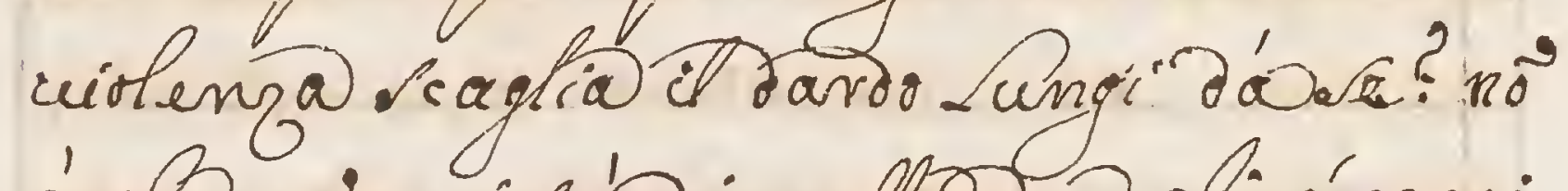
écho pir curri' a quello oud ali, ó corni cho formano du Lew, le quali vimañendo culaforno vamano viounaro al Aroprimo 
14

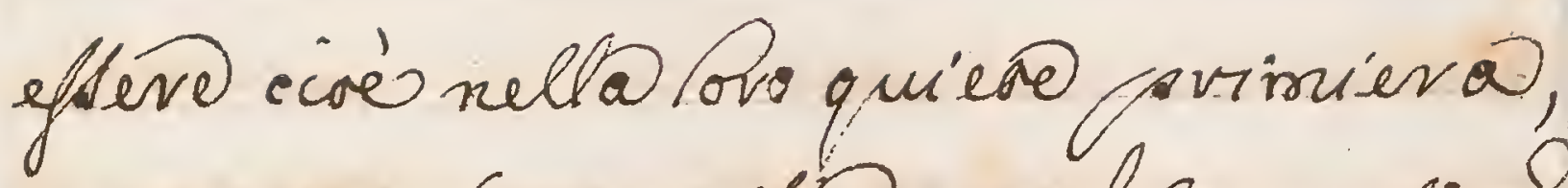

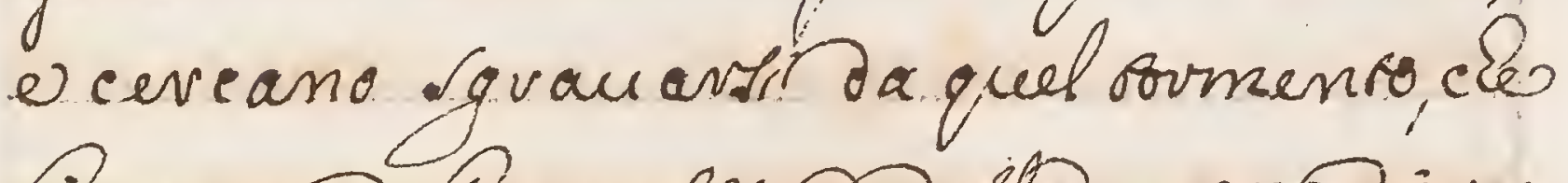
Ceagiona Popprefiónd della mano diuer rendoter andario al rovo cunro.

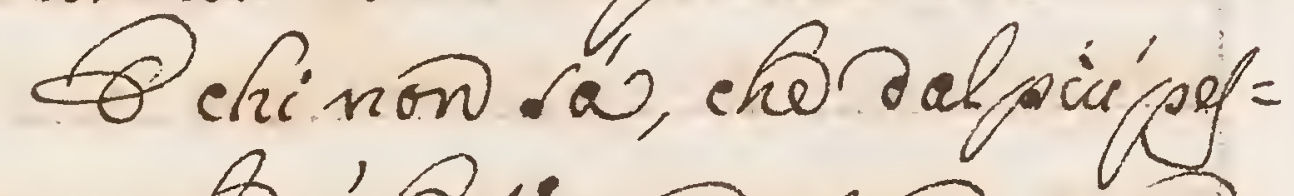
vimo culenoche' Ovipera señocaua per Tappunto per fýfepo wieno Pan: Roro? Dal conoratto di con pran pe: -a. Dal meastimo grân cess leno oven = defanima cerbar of crivito, ingoño

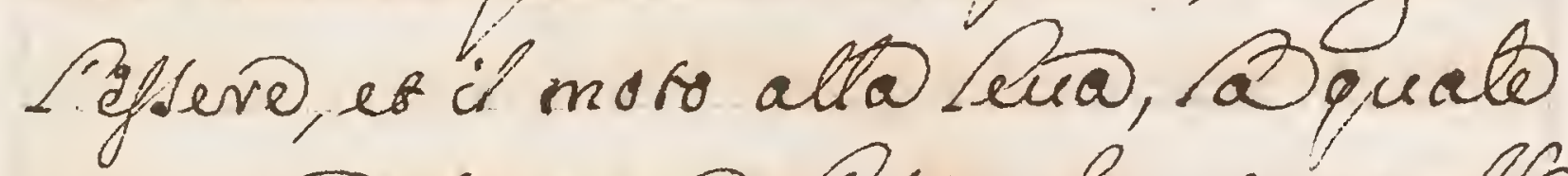

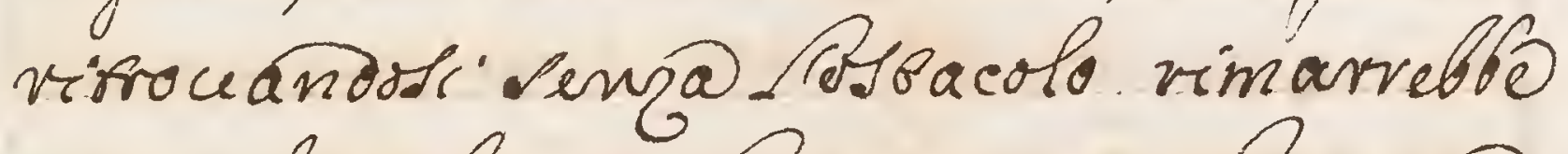
non altro che un Legro morto; Aunque aundorni Asingaro of for della Lua

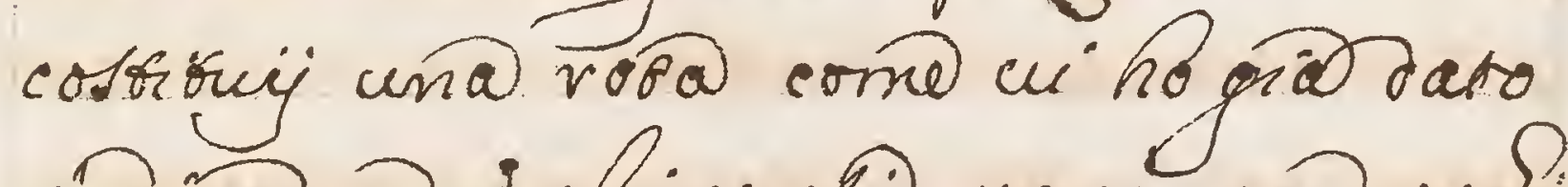

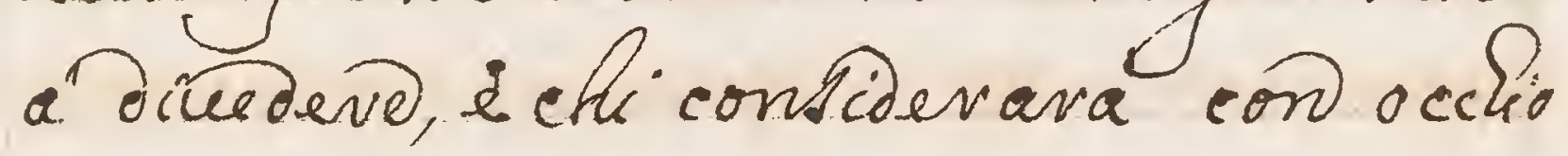
Jüappofionato ci vifroura' cén infini = a combinationd Lêd fer quanto 
soviravogivo.

l'como sgin'uns conovele, che.

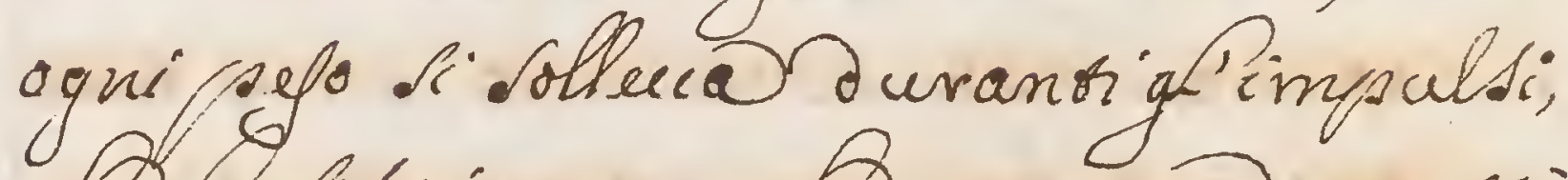
che sospingono, e che mancands quepti cieno egli preeciocitavo costo dalla propia quaueza, cotr con quedso cuevo cyppogito formai te Puetron vore, alle quali ando il moto due canasifoer crafotedu= na, Odentro a'questi corpi ferrici uivac= chueficon armafira, cheforma incugoro

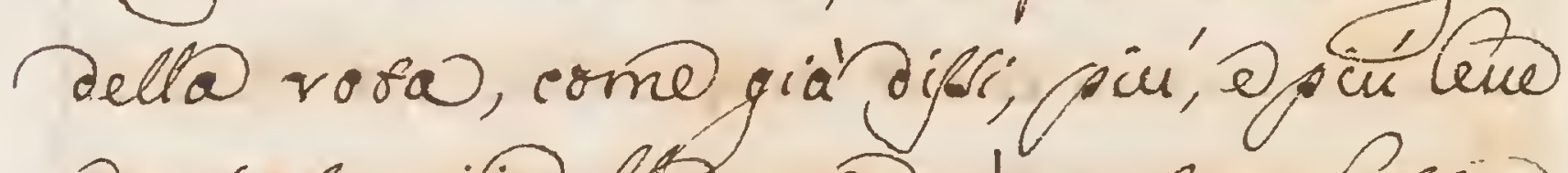
Oni veruig della vora a confemplasiono ckomancando cinapora u'Jotenryal.

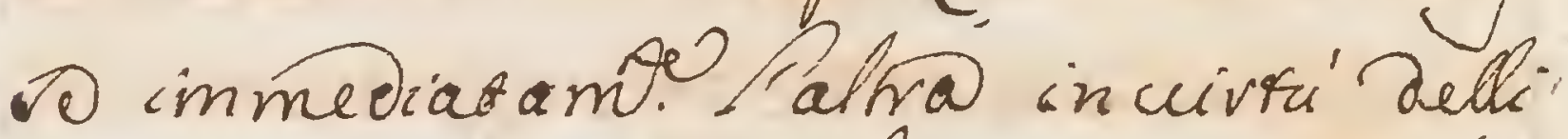

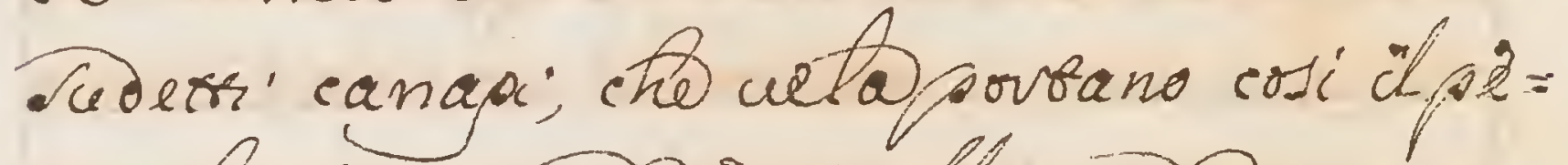

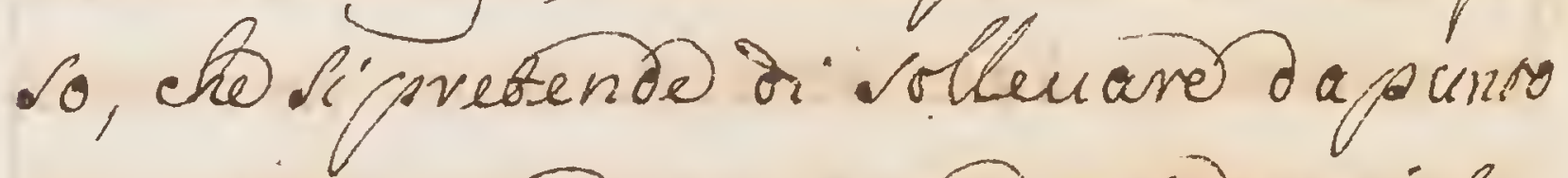
<izpuntonon vimangagiamaiden= 2@osegno

the 
is

Ro so io non hauefalyovima gef vinuenurogli un equicual evor con: Arapotro 1 machinanon our vebbil

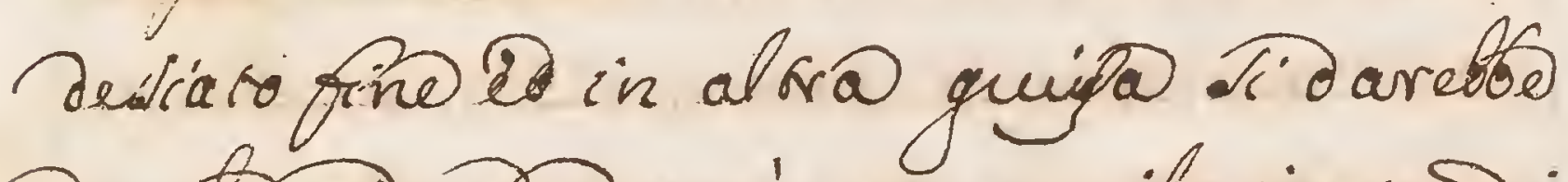
Da che vidervaggn'uno conil pianro oi chic Jeera

Oramifaró-Rerko dicevsua= Dero as ogni dofeford capprendero il

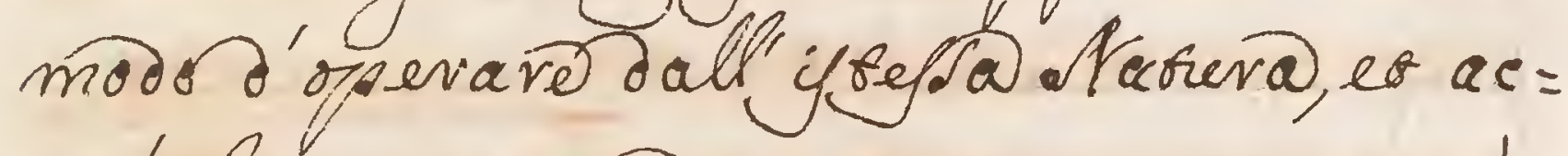
cio' che vimaniabe aps agati; cu' corbevé cio chardice cen Fijiso moderno nel suo firo invirolato = Msohoous curendifethep

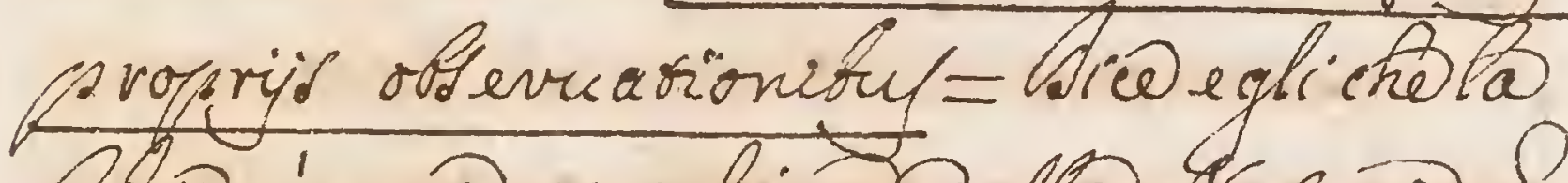
feove unamachinas ella. Tarivace toporia in campo cen rimocuevil co

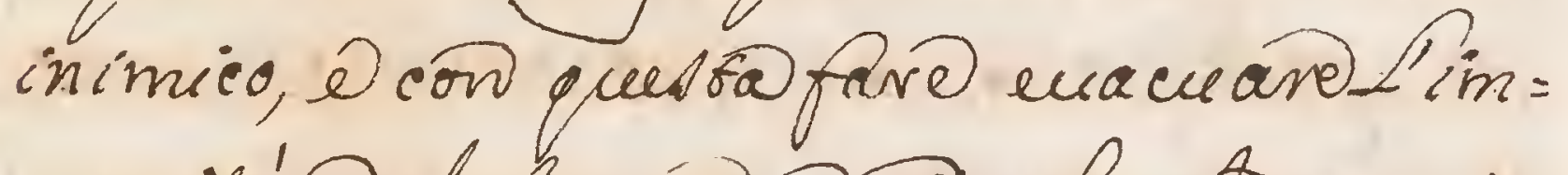
curitás del angred, oridurfo atoi por 


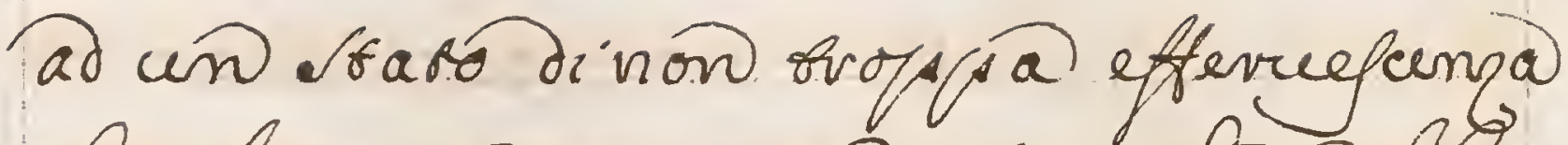

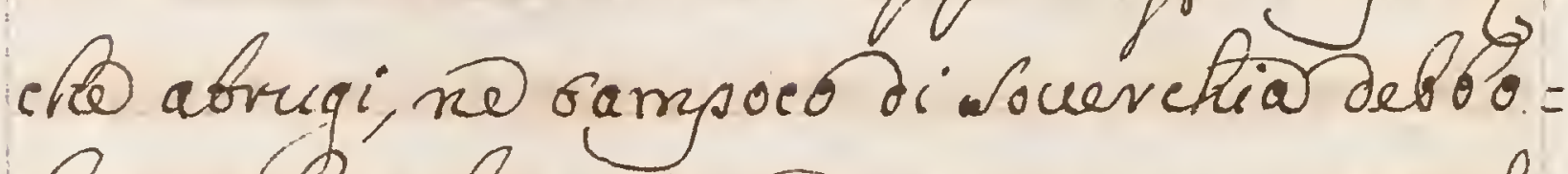
cira, cho in anguijica, conrempevandolo nelta fropleo gagliarda efollitione, conw forfific andolo quando divifroui nelli eccel= d'dello ordpro fiacchezza, e cosi vips ava:-

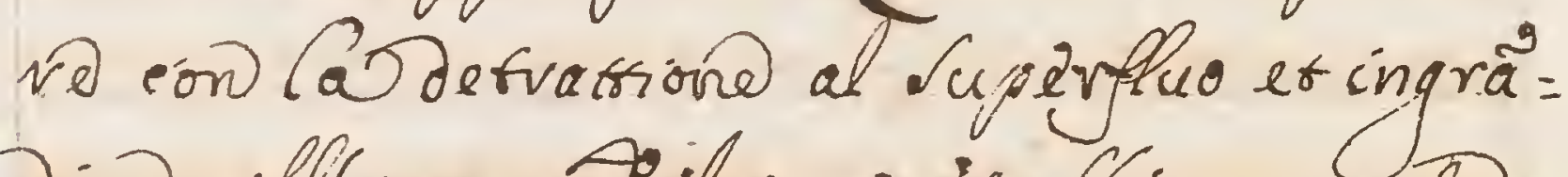
dive rolk auqum. il poes, it fippoctate nel Cifo ge decenoi ornate fol? 8.n:? propone al Medico = Abifieparata ario Ansorumenta, et Maching, et confe:

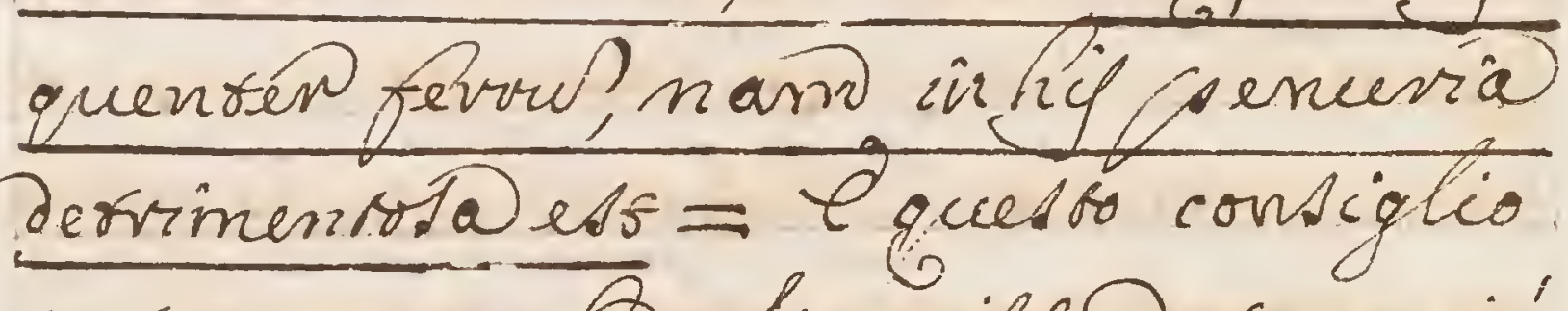
is Nuppongo the gli venifse dato acció chesi abtib afse a stave griardigno da. cofer vipavare con quessi ovoinniqua: Do ugglia al superflleo come al poce, ocenquequando una gperariono fole 
16

diuna.mifurata cioleña avả oine= ceftita' porte un freno, accio' che corriggen:-

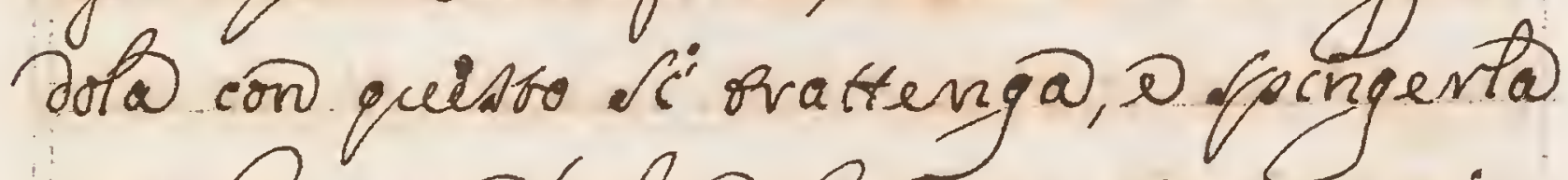

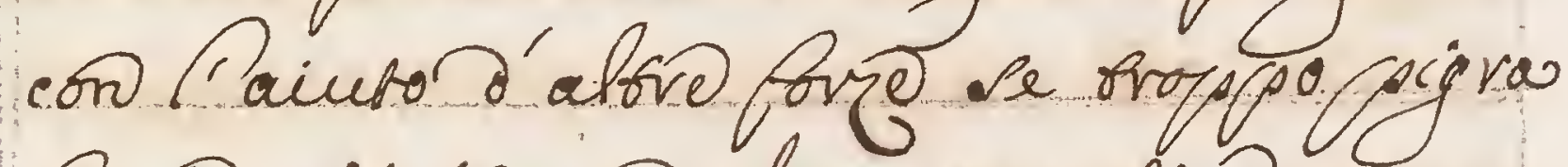

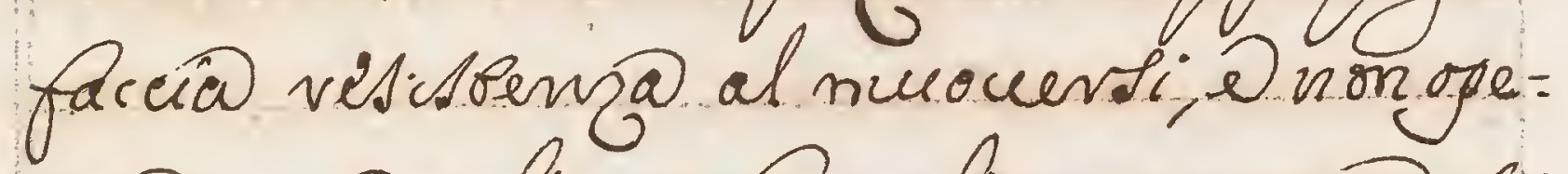
vare come fal ceni, iequali eon evrore. $c$.

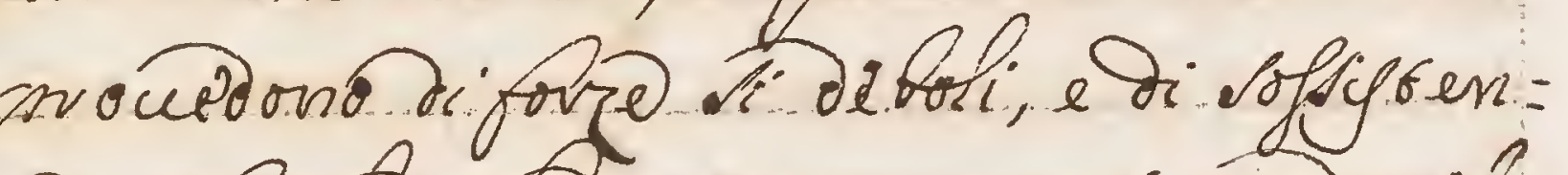

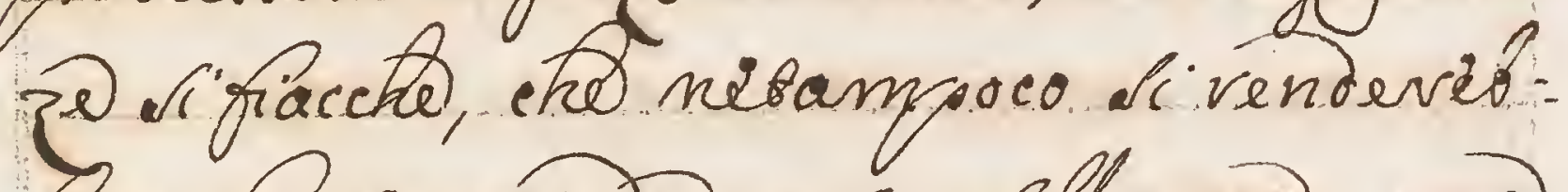
bevo Asfanti da poter slluaro una ciuma, ed al consavio molfi alfici, Puia = Rinón sicurano di ueveno candaglio, an: risengono, the fore fiasendenti yno smpro migliovi delle defili; con a vagione,

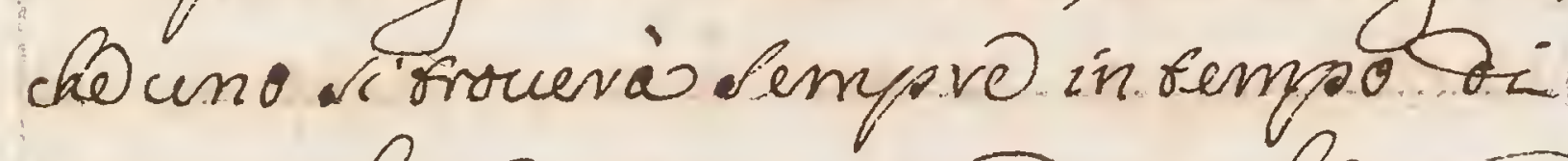
corregginta), is now si aumedono, che quefro satrolia cagionano of effiti del uento iquater ono ourvehiamencre, now of freob6. Taui al camino, ina al nutra: 
gio; cotí fifognavó or atrenevo chi froppo. andito precipiba, e of eingerio alturo anro chi

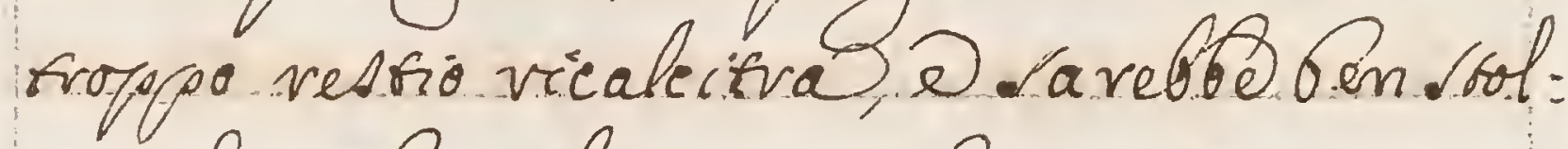

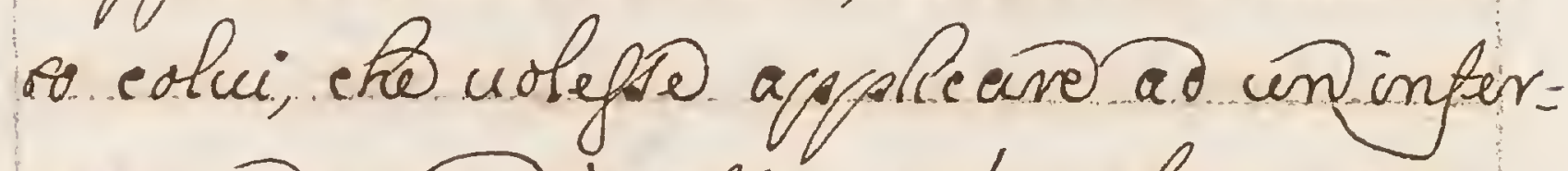
mo un vemed afsaifscú cioleno, aceno pui' defile del mate siela, in questo ma=

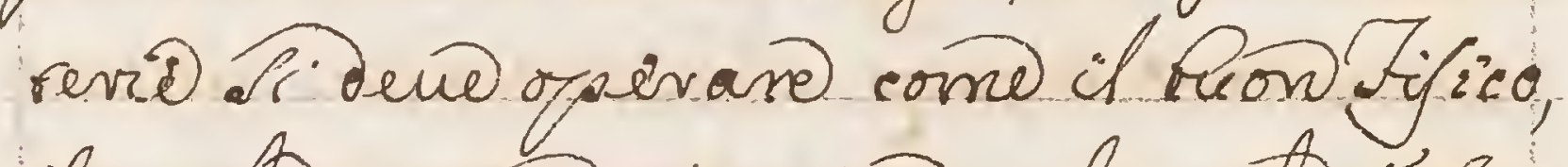
ilquale ovimataminanelmate i.in. tomi, exon coro mededimi medicamen: ricuraitacbiterdel ovineivio, il uigono dell auqumento, for for a dello to abo, ed

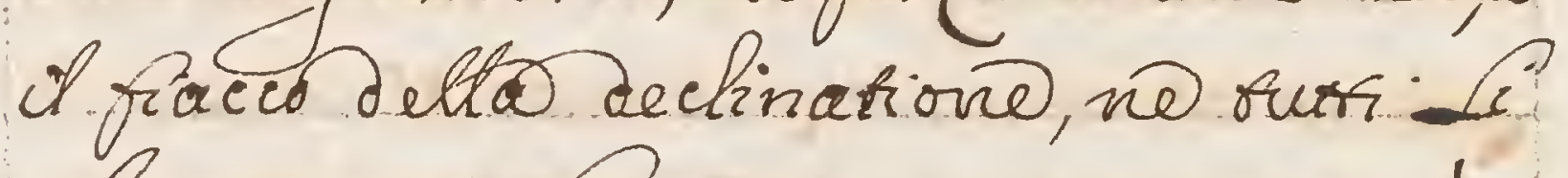
citi he rutfelo ustri concengono á futro Ieba'de gl Vomini, bifogna ha= uev Sempreper maftima che in $\cdot i=$ milimaterio non. Sewe avrifchiaro cola uirena, minove. arebbe paco Differente, hail cerderta, u. avá ame 
17

premagión OPcolpandon Señ vimi: vappis unidente íperiolo

Ma' accio'che apsiano zñ. berrogaro una urifa il Maenso dell'si: chifetura, colfura, sttura, Michelín.

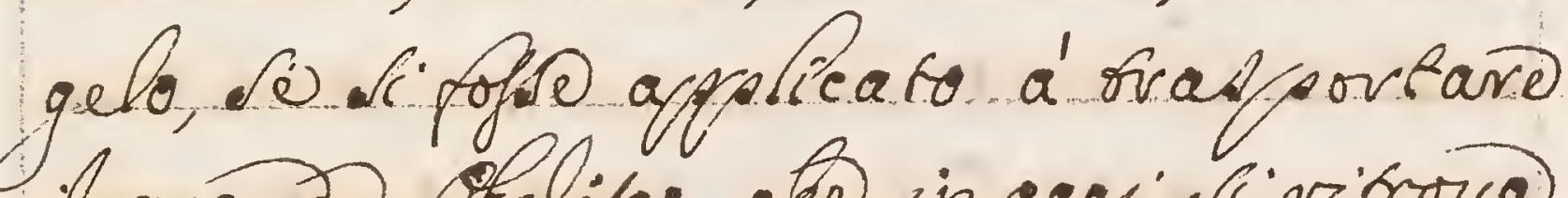
chrande. Ofelifer, che inggi viritou collocaro in mezzo alla gran priarza. Del Varicano, fíeghir civespecto nell. opevare, cho non rese alfia visposta al = Pormanda, the quesa = e Pedirom = Gifo? ono egli contiderando il presten = 6) anfiuturua il Onfano, a' choscenque binarogl occhi alla vagion allo ue= Duta cun ann pofibile, it arcadu = to che eghria aprivif poiper piangerve inubitni. io all commefor. Acenque to curuentiono avá impre a madre 
Gyisimadello. Suurezza, e chinon ope:rafte con un accorta precuntiug gl ac: cadivibe cio' che aunenno a' Jejifonte - frchifetro, il quate concepi A uafra idea Del grande, i fanoo viromaro fempio. Aiana efesina, in cui fo fpiccare gli - forr magqiorr aell umano apsene, macome affermakeruncio, corck un@uolfaglimaneó_.firpo, ondecena gran mole il ice injurabo po non coré vipovio nel Alogo fabilito, avrof

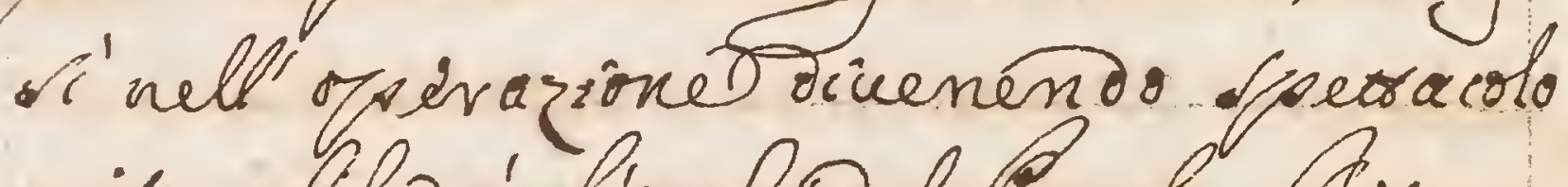
mijercaíle à of scchi del opsole. Aiaperasosi questi cer to uevquna fi in pro. cinco di srendevno vimero coerto mani Della horbe, o congunsam esimenti dall aborei rampogno. Ma' al cueder bi cei

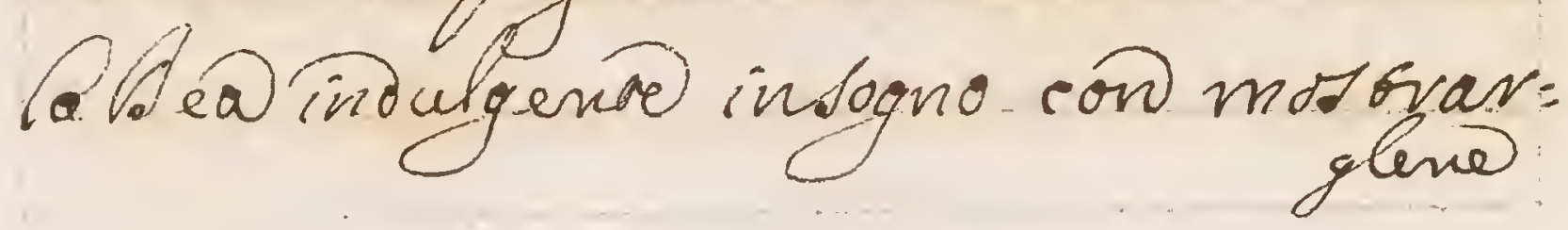


LCo

glemeil wodo o vaccondoto, ed egli cano: mizando Giculazions Li. Pogni notres: mi, iqurati al fine abro now evano, che

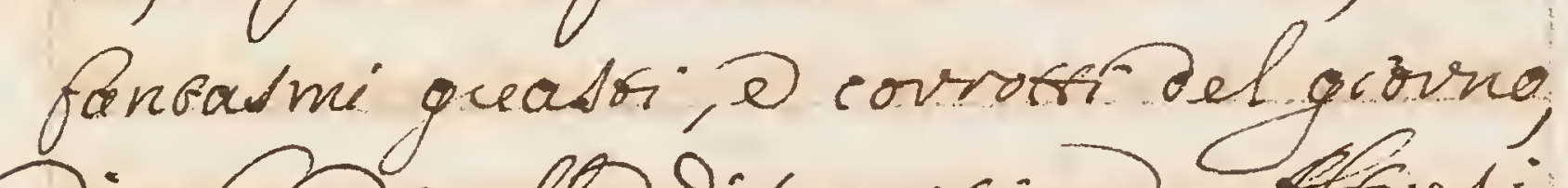
Die oando alla discervosione. fforedi.

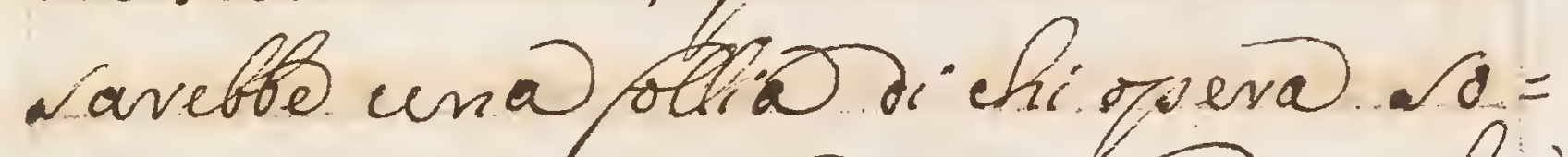

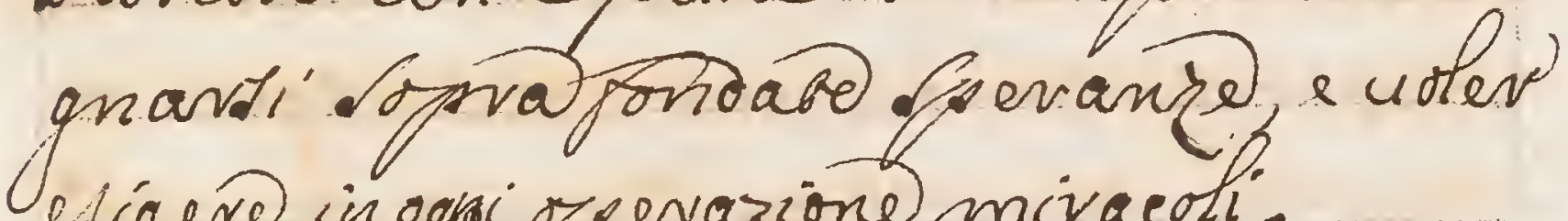
lifierd in ogri oysevazione mivacoli. - flionfanaromi ses ovene foa-

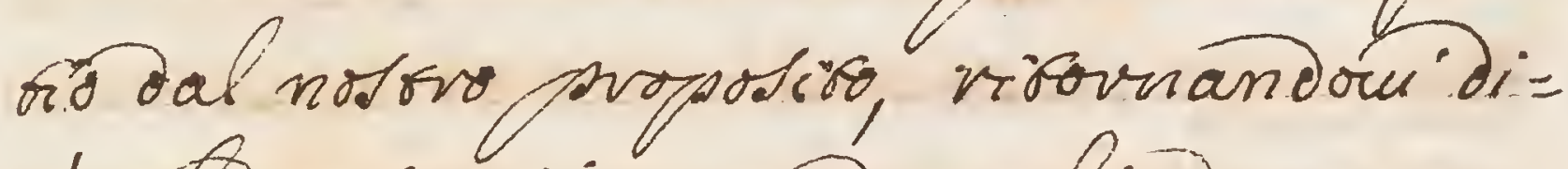
v', che coloricy questram mackina con que =

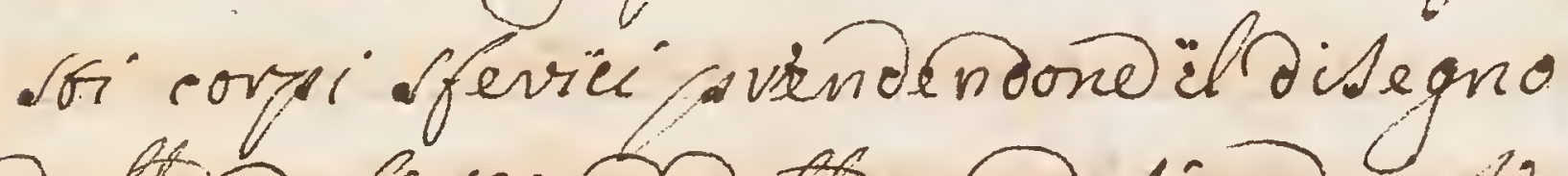
Dalsar definisione dets medesima inde=

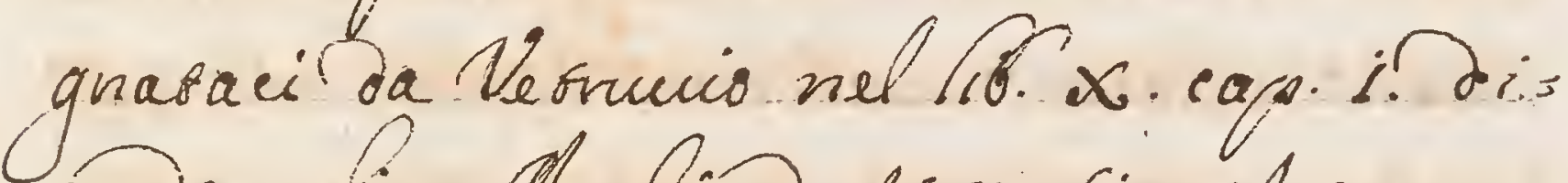
cendo equ = Machina es oconsinexal ex ma: Sevia conicenetio maximal ad oneviomobuf habend uirfubel = dicscí Reque, ecio cke

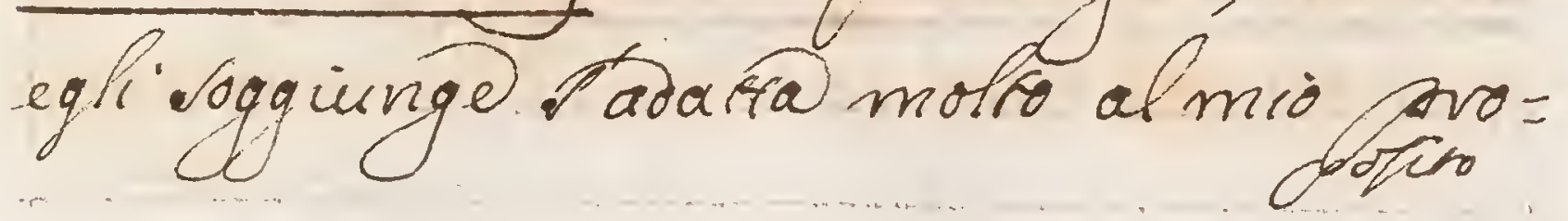




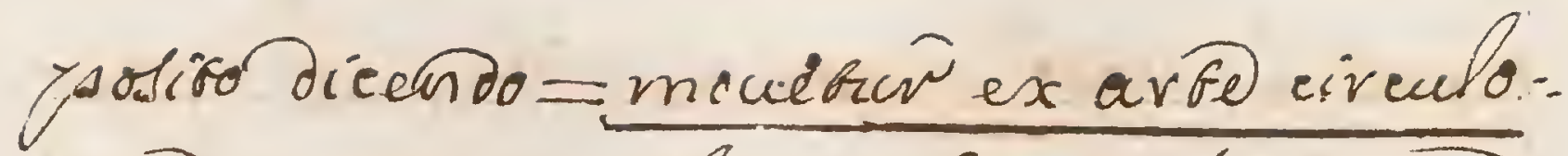
vam vofundationifut $=\sqrt{s} \cos ^{\prime} c^{\prime}$ indegna Cetrucuio is non mi arvo'aifongato molro Dalla.uadotrina, ofe equimi quegro giufro envimento novmon atau dall infallibilitál Aicina, nuenore alua intelligenza formo is globo gehesbe vofondo, e costiou il mageiov flandea con sinit igura, che cirono chiama= Aoraferui: D. Plis = insomma rutso ecó, ehorivimi vabicinro a'cifur ro ovo in pero non vathie= ne alla forma char votondita's, onde. Alei: no firsto fo Sironico veferento Popinione

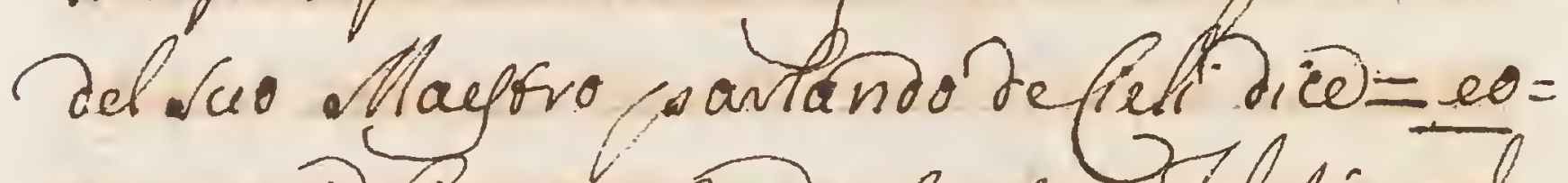

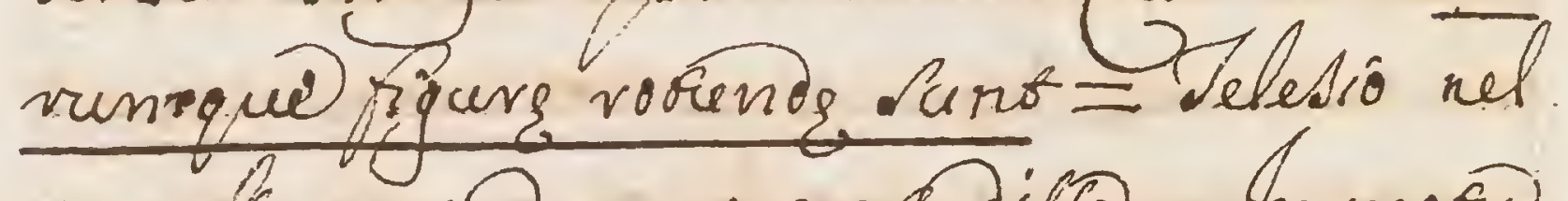
convis. cap. De ver. nat. Jypo = an morw civentavi 2st faciliug opevasio fic er tri:

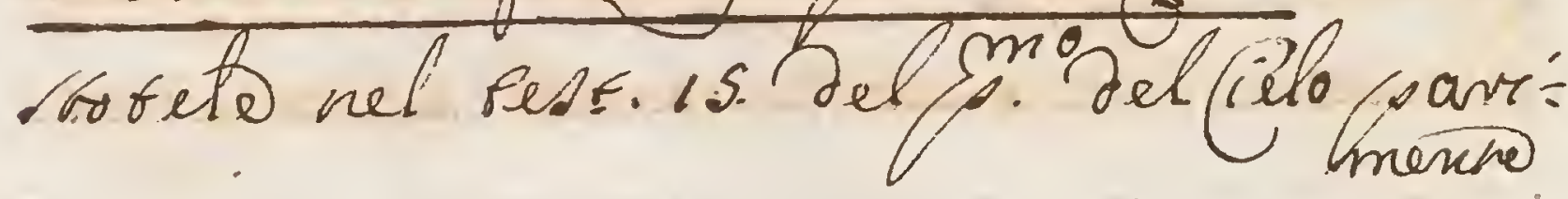


19

mente.rice = civecemlata corpora curlen =

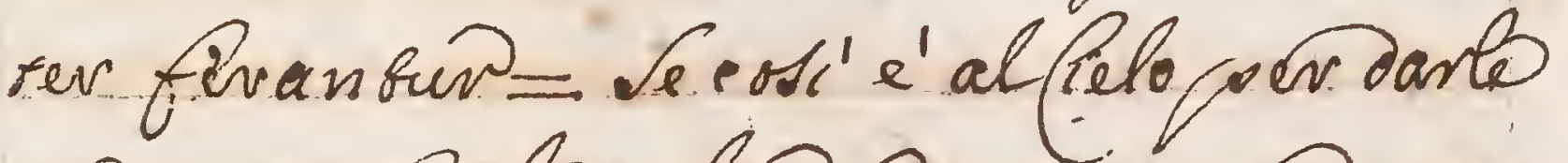
un mor facilo sehere, opvisto non pe: ruariganizarai che fivico, es ancho $B$ ilevrafu eveatafferica comefiguraláiú

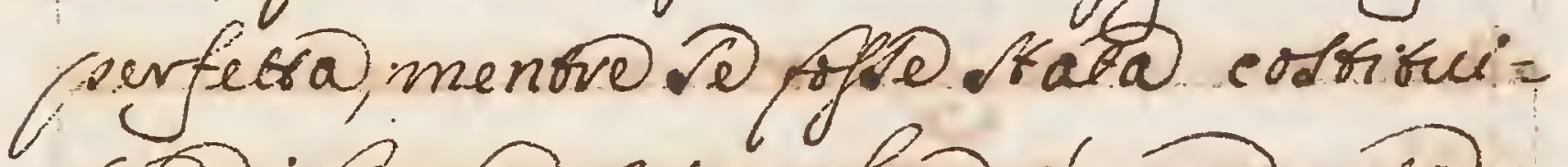
fasdifigura oriangolare, ó uadrarag mis Lentimento, the cequirebo per ne= cefsira', che geni grand il quate desteva

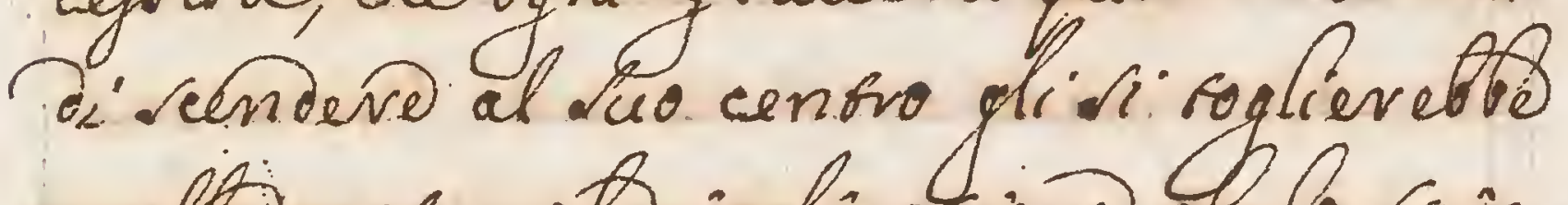
quelta narevate inclinasione chefo foin = goal medesimo, etaefendo altivarge= ra, chovoronda, now aguolarebbeil mo= re, ene accadevelbo como hogia detro. che alune anti potento rane alsuo centiro cuicine vimavebevo per 10 vedijten =

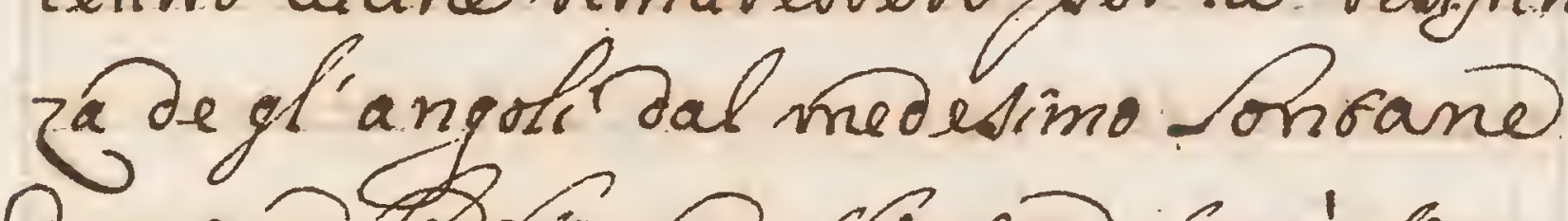
JunquePfigura.feria dara'.jem for 
pued maggior perfectione, 2h. form a

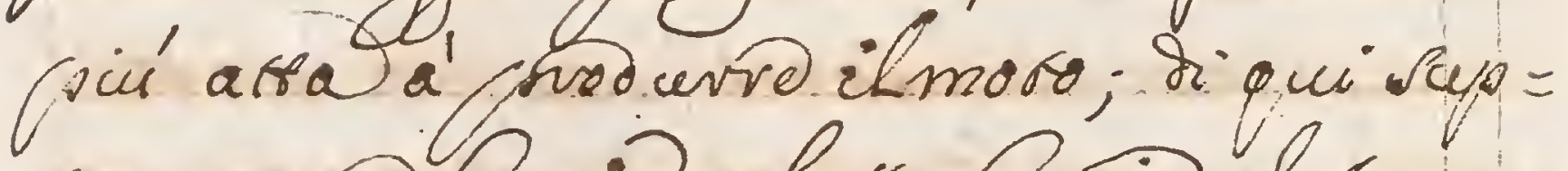
congo now hawer colpits Angi dal segno con acpesgliarmi a' costionivo in quesso

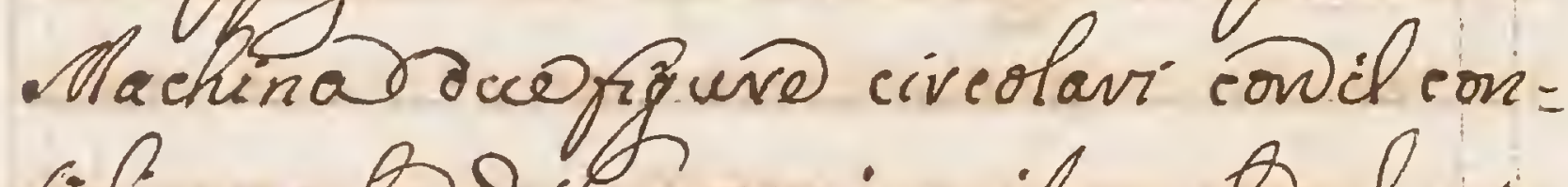
Siglco anche on upernico, il quate al cap.

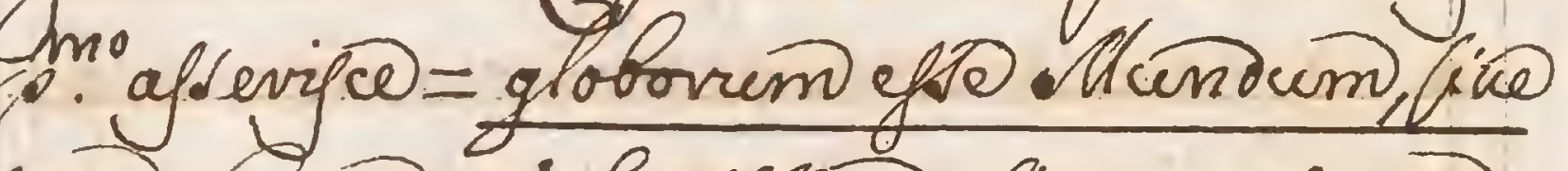

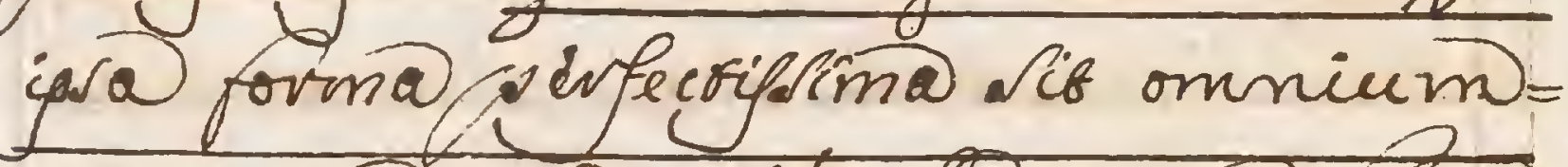
agquingendrui dicici, che questo thote Ganno Tficior aella Leco, vide me = aefine non mougherv con facilita' il pefo puefifomi certs che si mir arebo uno impopibile, w renche 10 oura/a ca: pione onde. Siginevi it moro alla me= canica, friforete bon cene da' il tume da contidevarfo, dicendoci consjprero nel civeolo à cu eglivalde 10 Fitancia á puyfail Vere, al Netio Fursigl alor" 
20

- Fromenri mecancei, of fificavo il mio. Sentimento ancho con il paverrod 'orehi: med, iquale moth che o cotenjad Vetreliol pelimo qeneve, codi cavás Da conctuden Sempo current aubio, cho

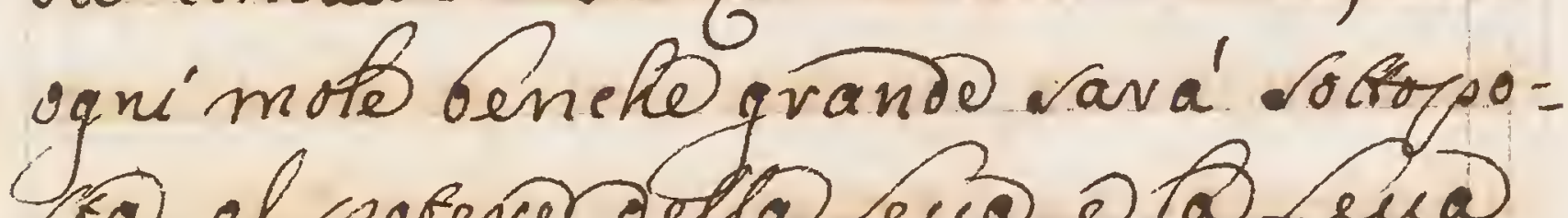

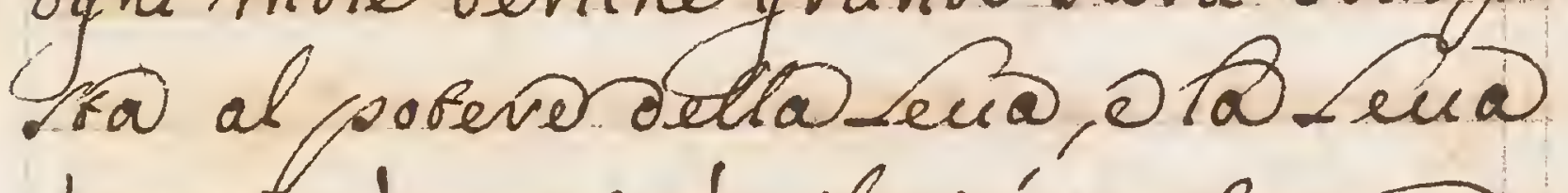
évarab, é, usavó il ficu' perfecro ord: = quo del Mondo pofsaro, del cuetente, Del fifuro, ed aueva' una coropowsio = naba magio da far comparive mi vacoli.

Airomavó a dive che ho egofi: suito qusto rofad iqueso gran Dia: merro agevotantli il moro, e seta ce =

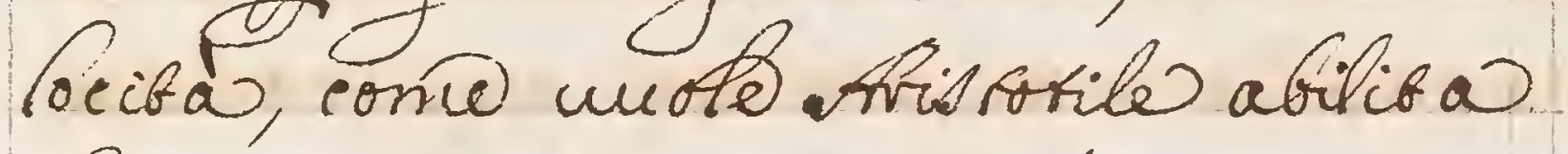
a coteña opevaró auan fagico fara' anche i, eho molfiplichicen mag = 
gión poterve nel bempo.tgls, che if $m a=$ bifesereitara'avariytenja, ripui

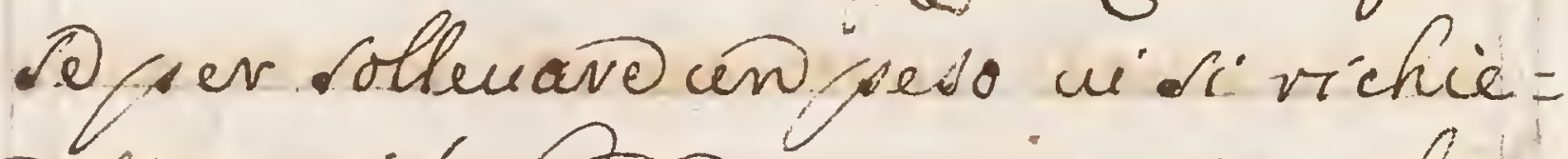

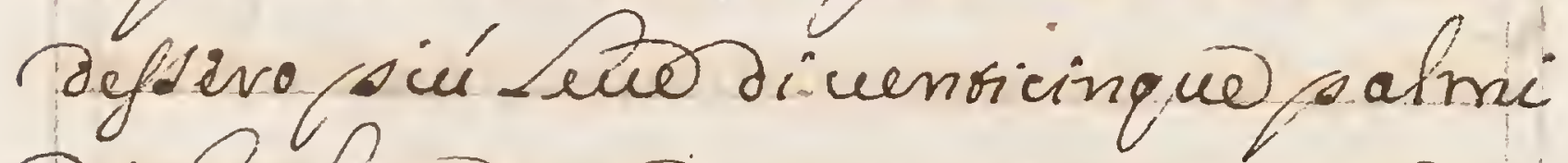
Di Onghezza, con un accorbo proued:menro formai queste vore le qualivae= chiudono in Do trefo un numevo inde= ferminato di eut, ceoti ogn'cena di pusfe haueva' un ualovo inforidseco Dimolfipleato potenz, a neorcho ogn' uñoiglsnontia, che un ofo istro= mento, come appunto in foro compotio Dicen infinito numero dípertone, no mote Devei, más formano eon un arpificiolo Ligaminto una Sta armonia, e queste vore applicandosi ill'ato ai nuceuevo Siviorouavamo incefornteminte ponte of aramo 
$\sqrt{2} L$

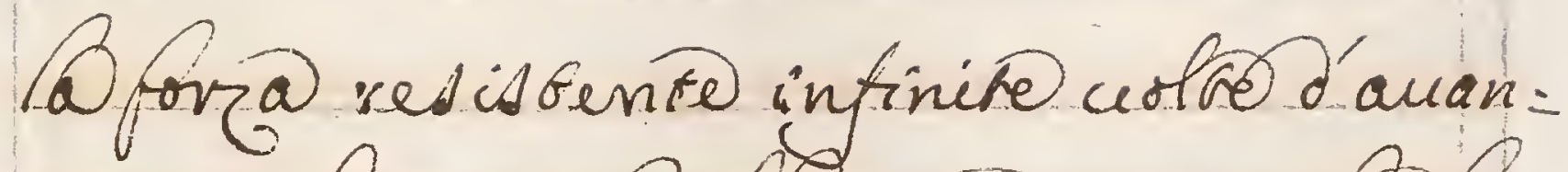
raggio che nonfaribbe una demplicele: uabicalmi unisinque, anchonajo,

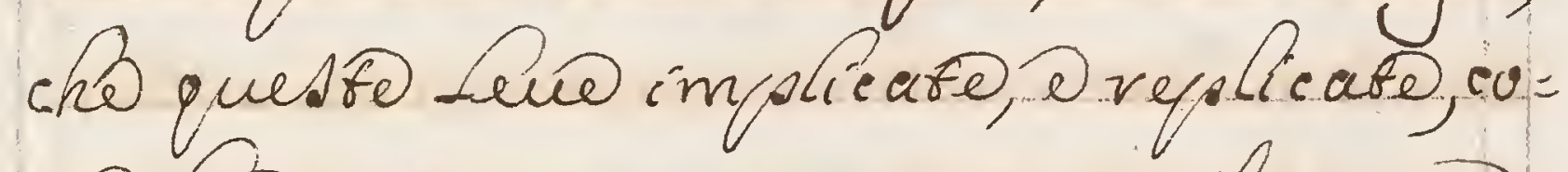
no holdto, concorveranno conch uigore unito da' cunto in sunto, e con lovo cotenjalligata ofsiemo farams argi. no contro vesyrenza del peso, che hi cretina di muouerro. Tornero' anche a' replicave che to potenzo in quisto vofe gli wien cagion ata a a infinite lue equalm: ffricici, o queso movendsi con incidenge perpendiclavi communicats = idalta comprefiono delle fini che to vagivano no portano il moro fino al centroldellose, quate con 10 for presbaraglia alt Lewo vaccoghieño in - Bfinisollua il pefo prefífomi 
Dova accommodaro' per ch notoro povopo = sivo una erreditione, cho. ono per nar =

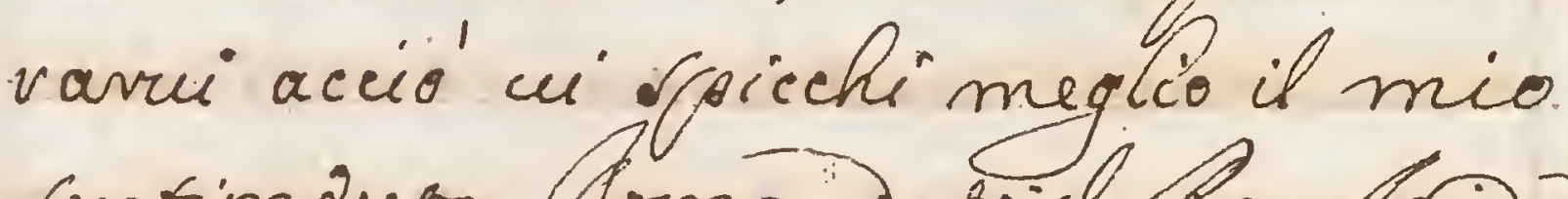
sentiminto. Aomandato lle tgido quansi evano i fuor foarfani, che mi=

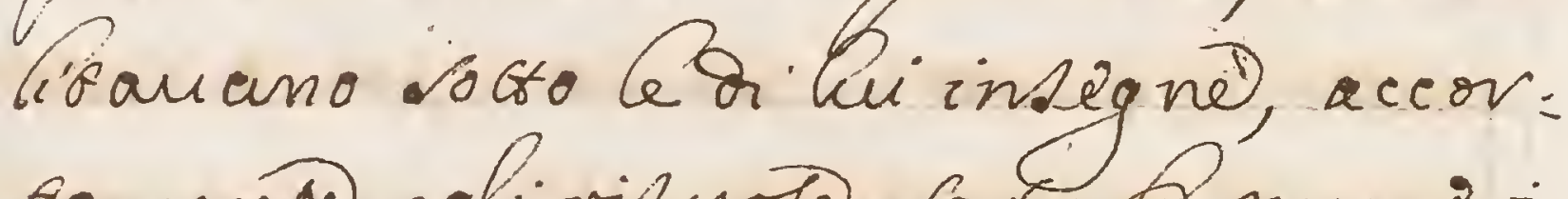
famento egli vis pode; a bu to numeri fiparranno ben pochi, ma' Le f'abrab oevai a'viguardarlinel conflizo sem.

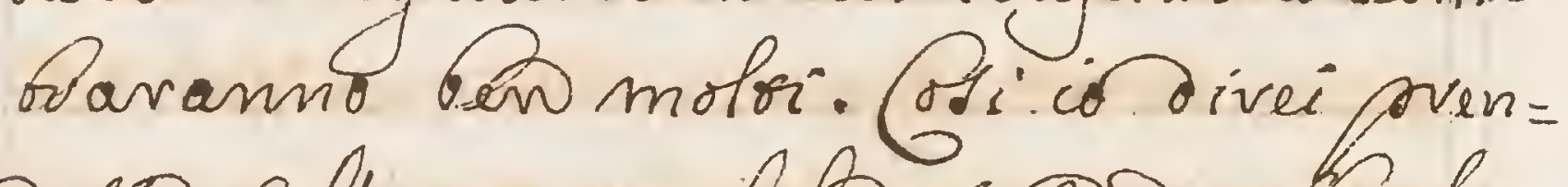

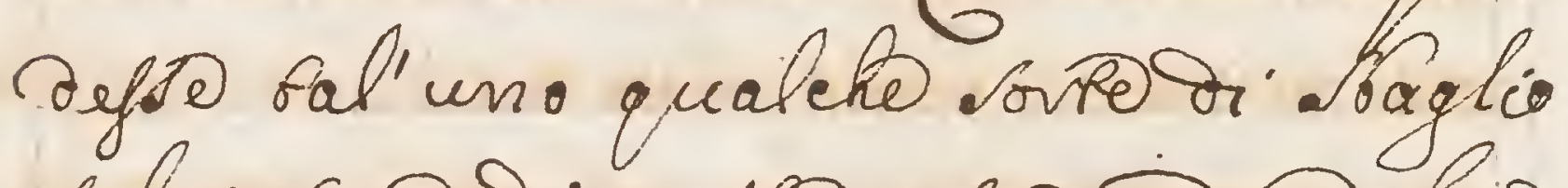

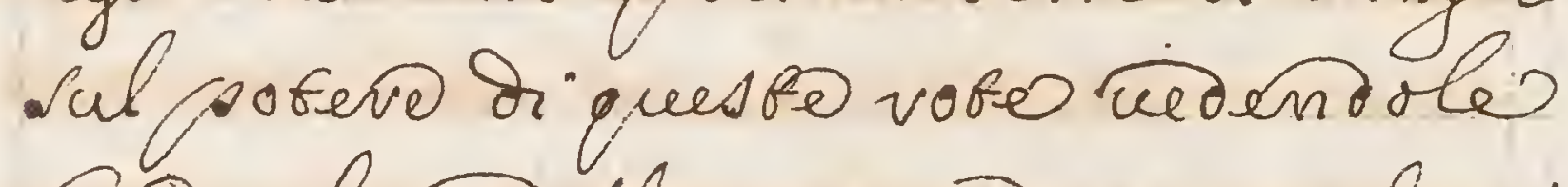
forfo nel modello opervaro, cervo che ci viconscivefos quel santo diporerv, cho firdono molro for unibe aftieme. - io uoheldi fave una digvefdione fa: rebo di mestiever oropso cun = go Jifeorso frer oraraver zicio, cho 
$2 \Omega$

¿'éda' unviso nel mobo cosi' ael civeolare, come il perpendicolave cosidel verso, come il sarabolico,

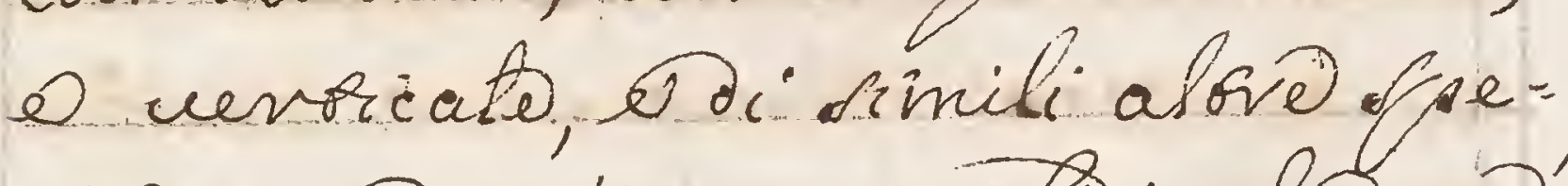
eie, come ne'i corgsi otidi, che no'

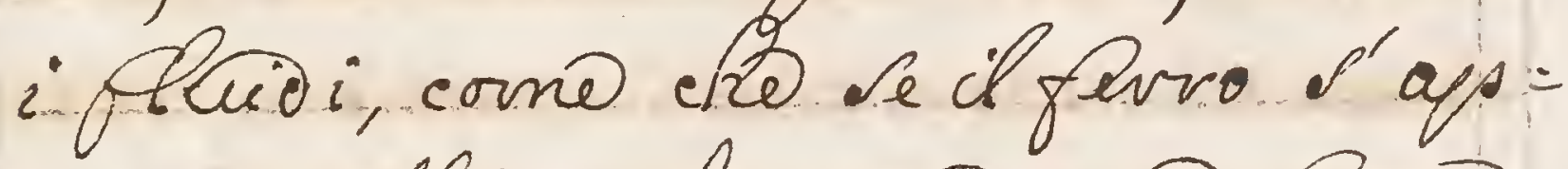
cuefri alla calamiba per frob -impraficas, curo sen amove; - Quirtí vimanga najcostaí quel.afo, ouevo na nel fevro racchicisa; Se il fevvor a per se alteg= - Si muoua, ofuñall am uara pieva unga rapito. Oo in que ro parficolarer divo' accordaño.= mi col coareroti faluno, sho alamitaraceftcedendon i pori, equetis Sehievas in Guona ordina: o nusso Itefles magnerico es alano 
alisi foirifosi, Siquali oram anda: si uevso ilferro, d questo nong poren. to vidiffere à quigh aforalfi; merceces

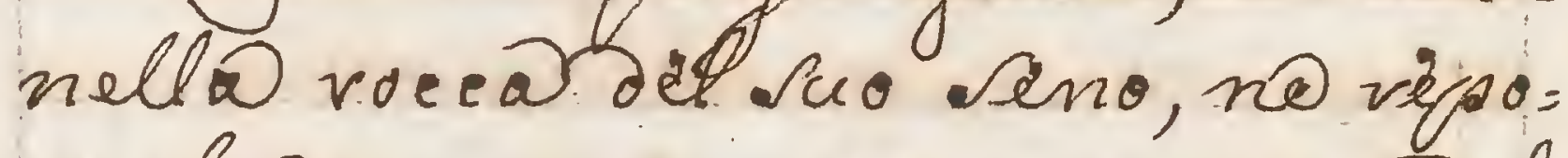
vigli to aur povi visvound del= Oparti magnioiche confuse, mal composte, fora foro inorieabe, dal: Do mededimo fradito vimane, ed ef Sendorella medtima pieciér della Qlamita conviri ek ada alla orgevefari quelli aliti cigorosi, o civeonuallato das quelli piviseli magnesici, o simparici i"qualighi Seveonor di fermento, D Po pongono in moro coti viene finalmento da quelli Sradrina to como il Vinro dal Vineitore in baccio Della alami: \%a. Má mi viserre ingenévere i' 
23

mori a'farro cen cić corolifso, el:

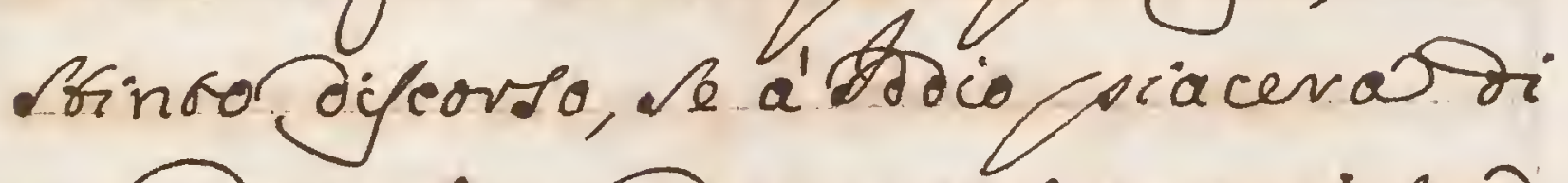
concedermi unamaggior quiebe. congiuntoroquathersirinag. gion commoditás pis avno poi con altre matevice curvios qualch piecio Paggio al Mlondo

- denza prendermi Ba fariomaideseriuevs il casfello, pianr@oel mededimo, il prospes ro, como ancho Tarmasurabella Olonna, mi do a' credeve, cho quefs.

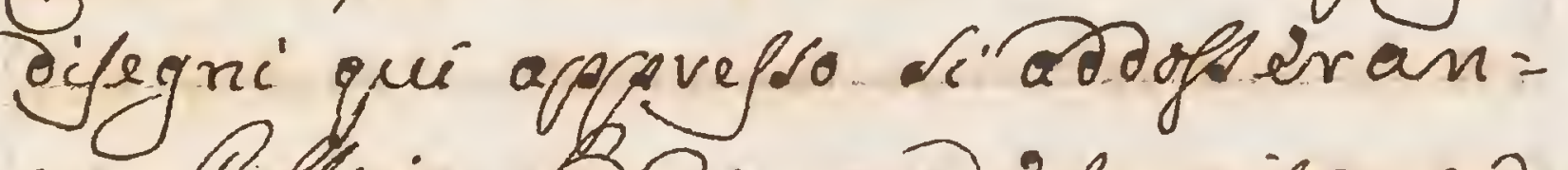
no Pofficin chonounaterveitares Qpema. Tanvo sicé che c'amma: essia acprefto Prodoro $a$ arsten:

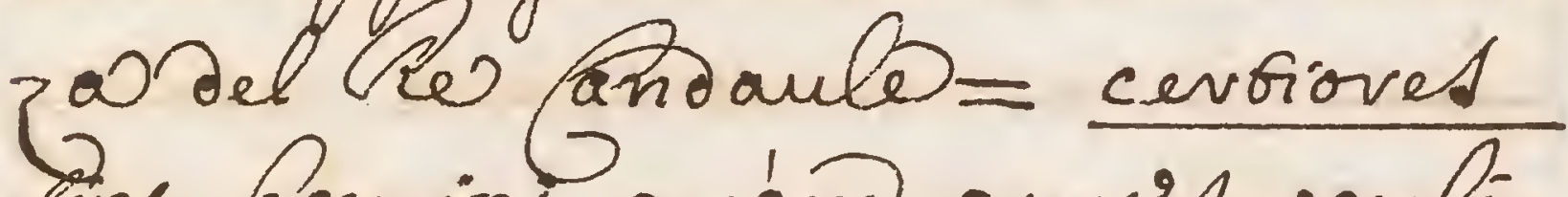
ins formini, quám auris, oculis 
2) come cantó Foratió

Segning ivritant animg timiso cons aurem.

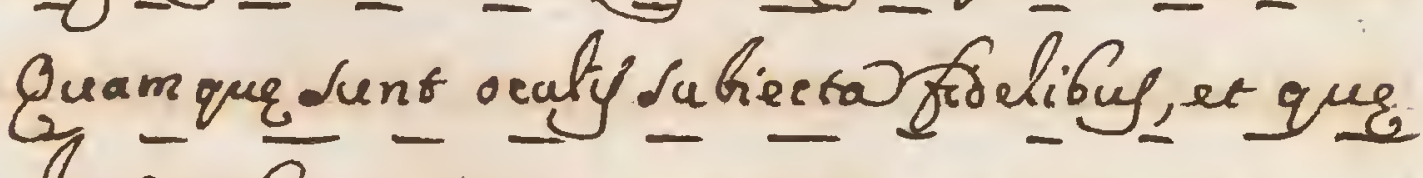

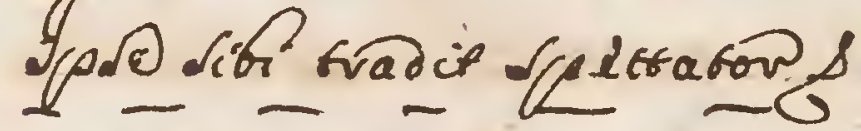

condupposto non accadenán dimotuar.

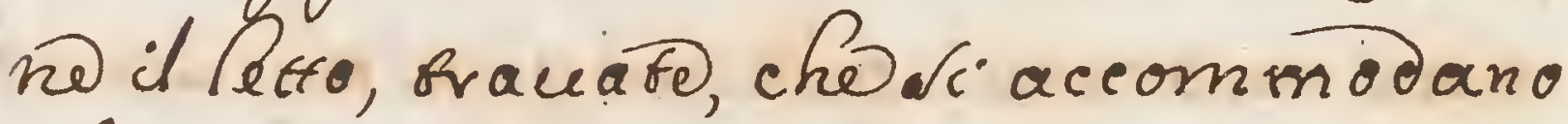
at di Sotto del meresimo Leteo Doue ua' àgiacere 8 ofonna, menore $\alpha=$ no mafevio egginite nón dolo adogni curofylove, ma anche a ogni fabri icevo benche wile e coer oral'effecto in questr non ho fimato a' propsosi so covolongavmi per non veno evmi Pedioso à chi Segge 


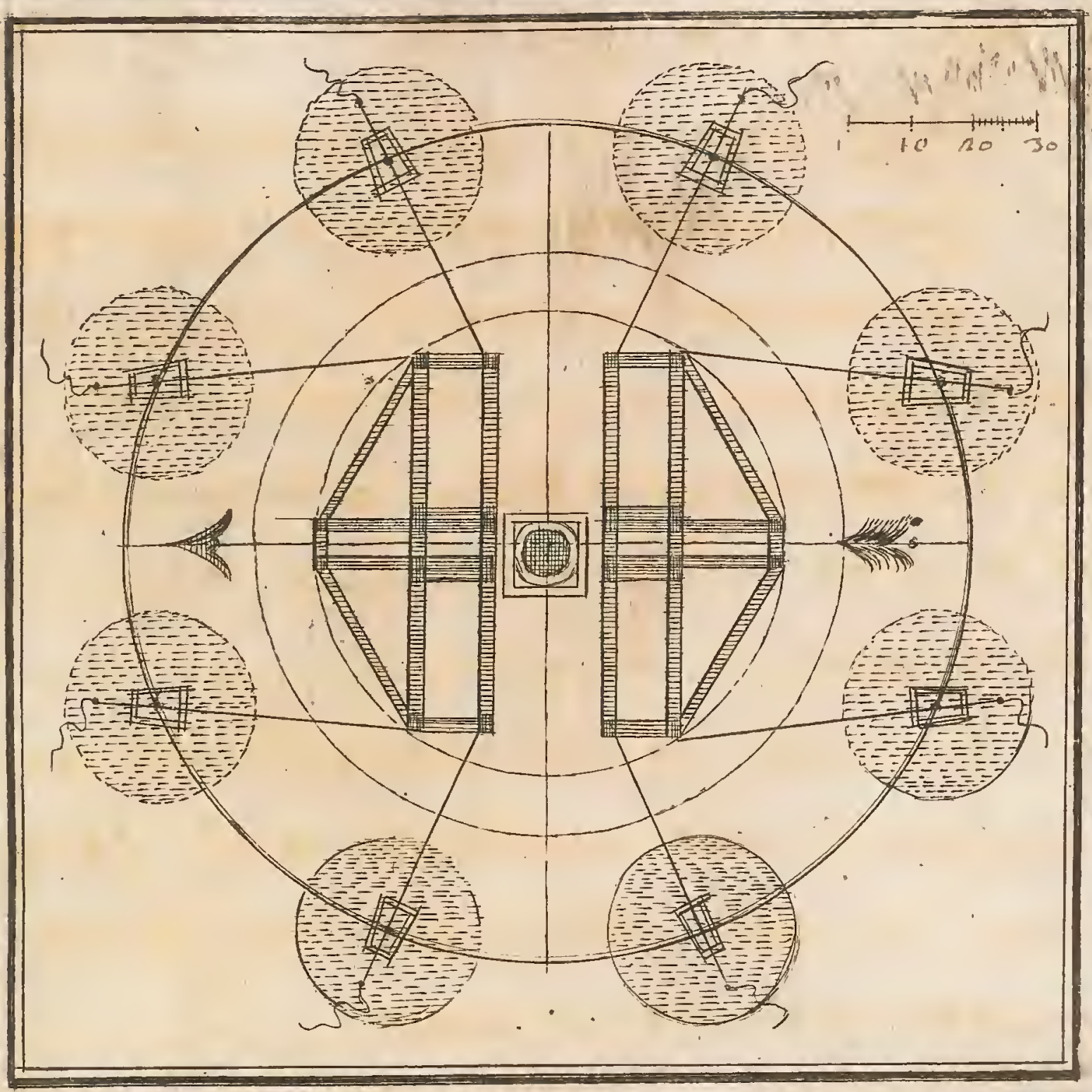

Piarta del Castello con ruam os. Angani 



\section{$0+4=-6$}




\section{Profilo}

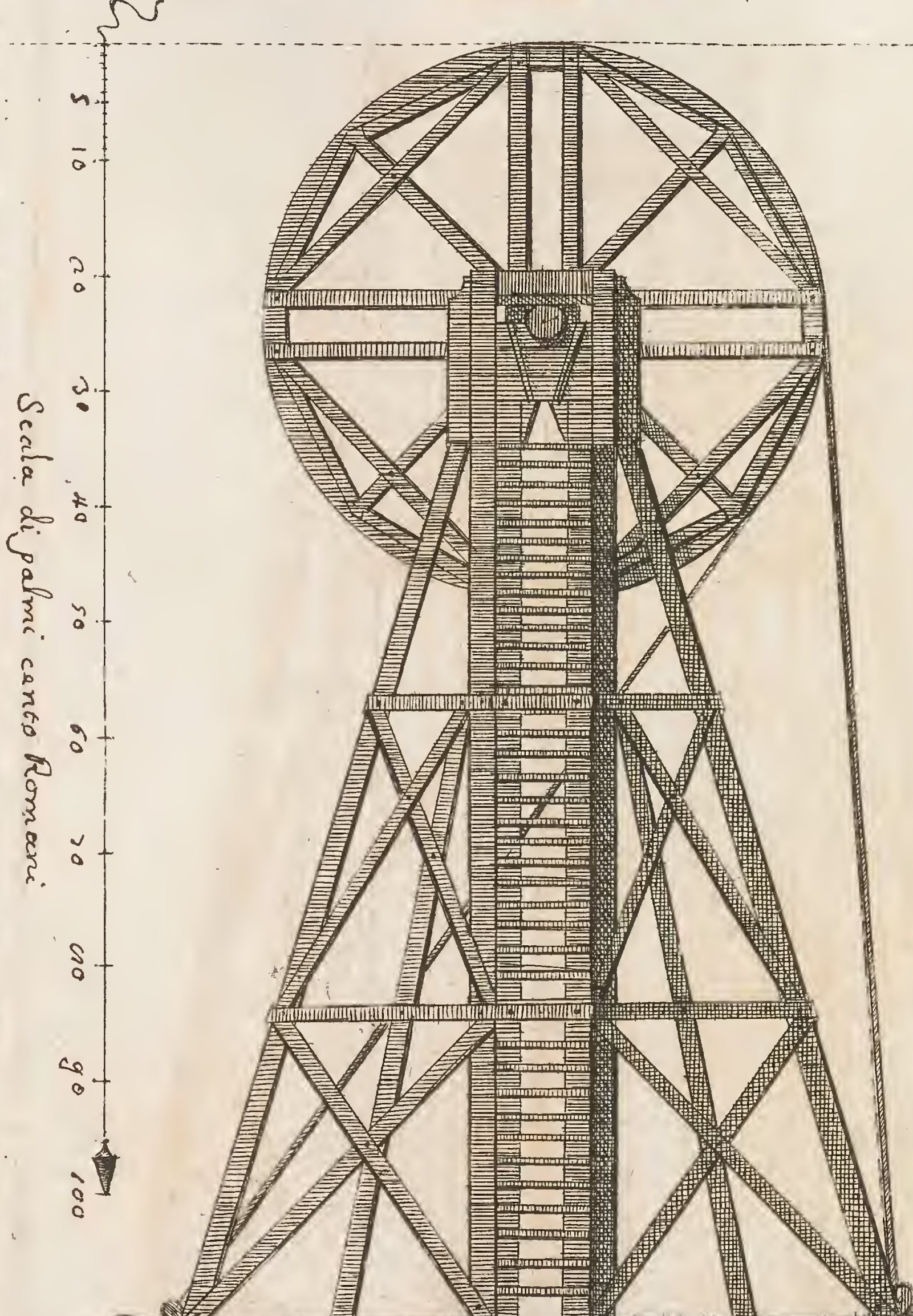

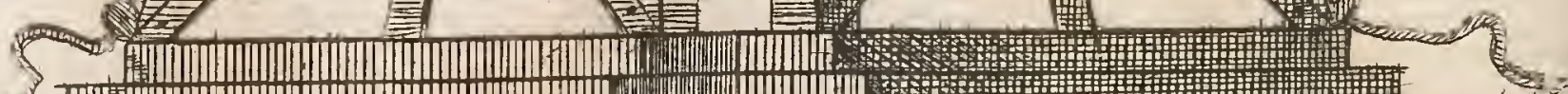

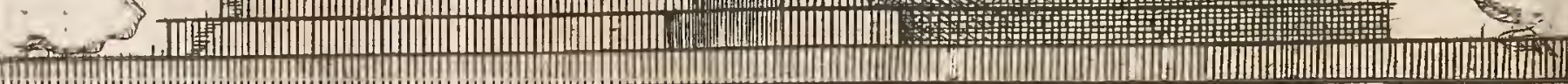




\section{Prospetto}

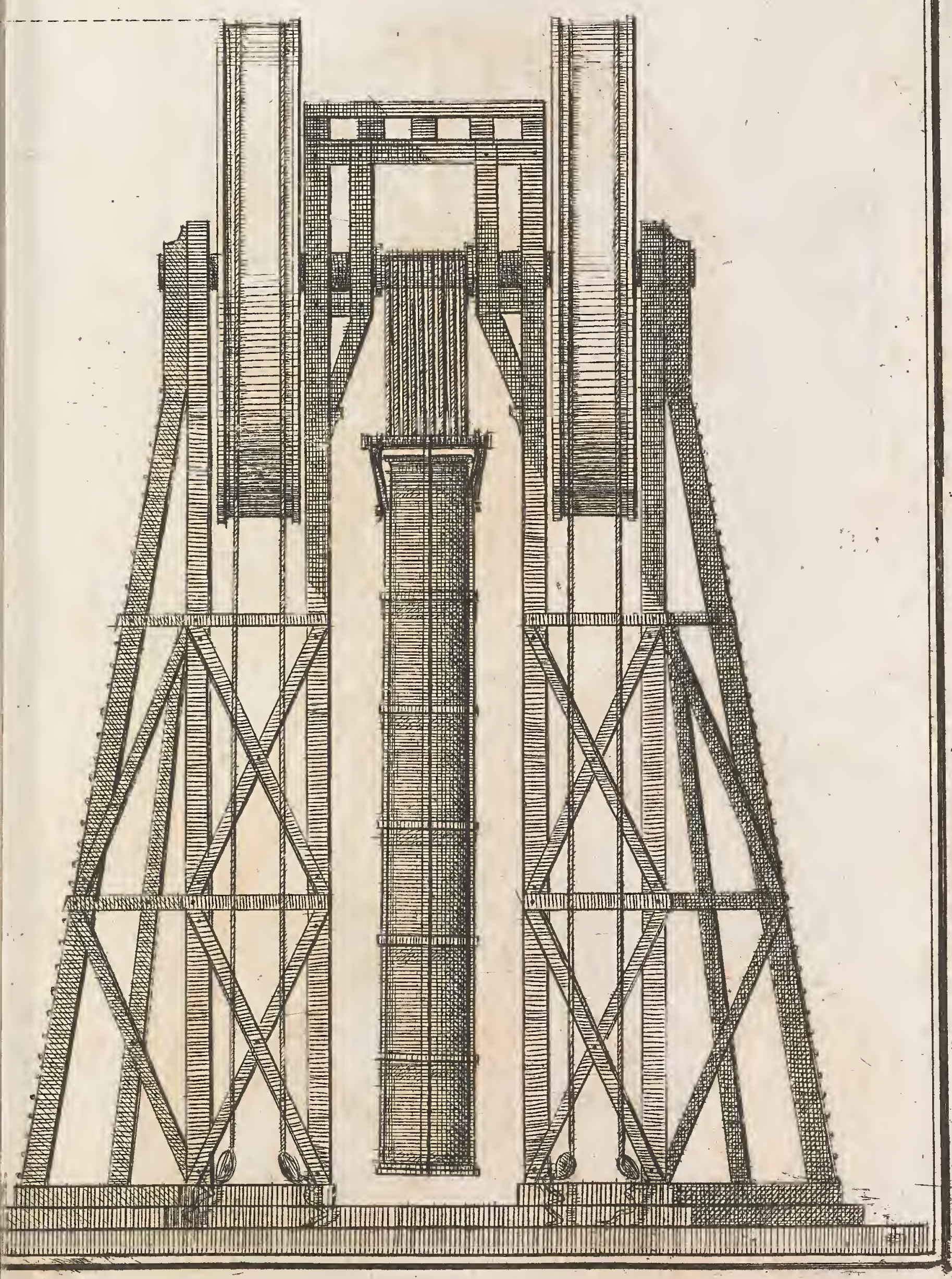


$+4,6+7$

8 


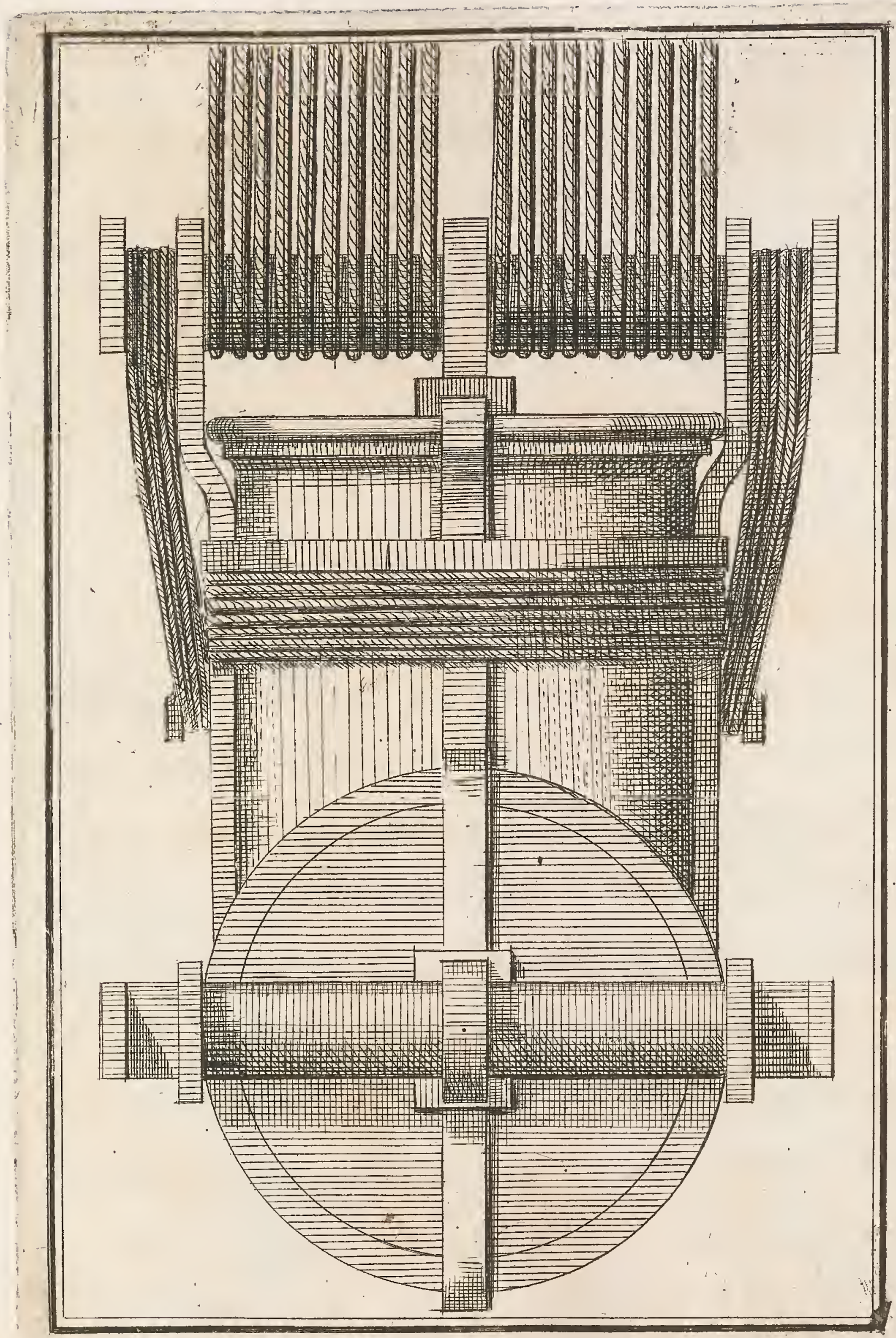

Imbracatura della Colonna 

27

Afabbio ogn'uno a a Sapevo ch Popeve palkova non sorfifcono un

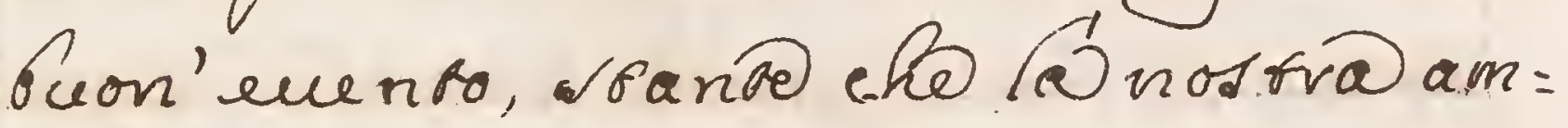

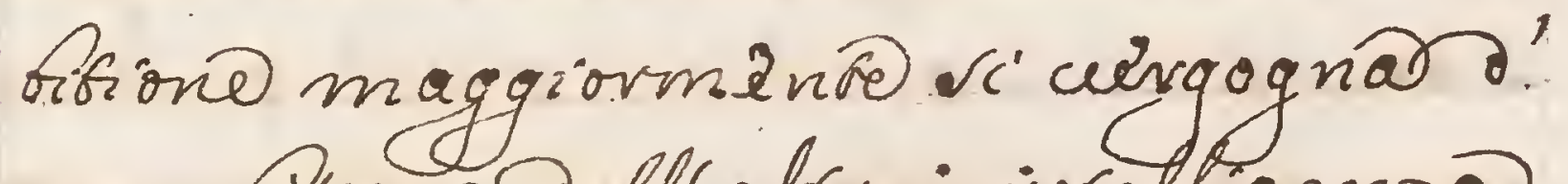
aver bijogno dell' altrui inzeltigenza çonow valkgra quando@ vin: uieno, e quel ch'e ti.erggio e' ch'ogn'.

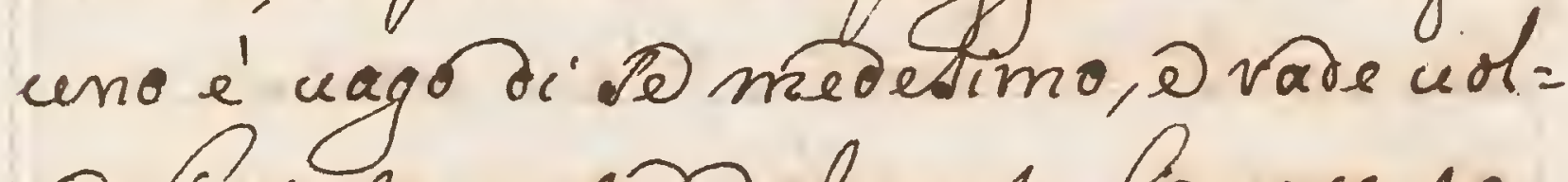
bo Pricuto najerdal conseglio, areso. che noi non ei Sommetsiamo al ngtro capriceio, e cifsensciad maggionn?

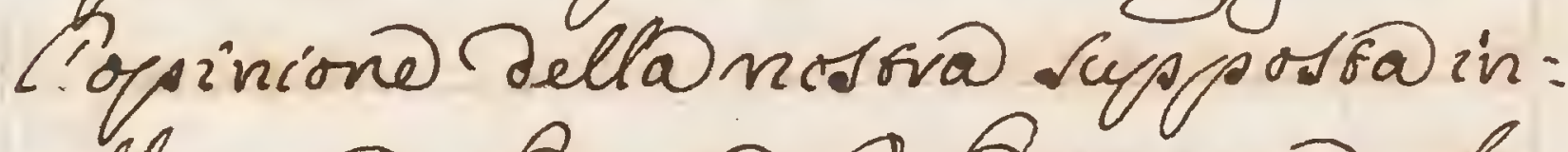

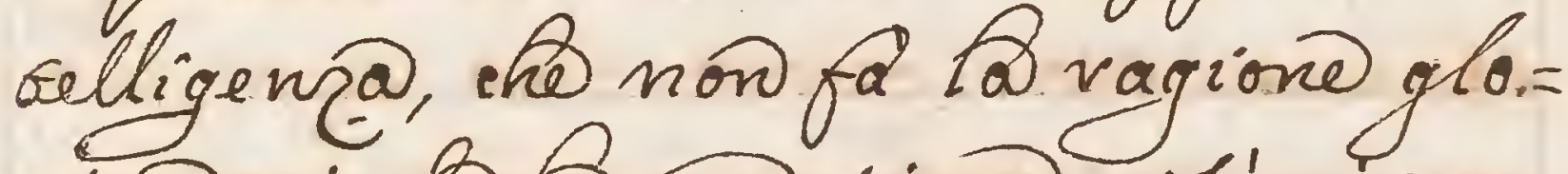
víñoci ch 10 medesima cosi cifper: riadaferiuendo a deblezzoch? alfruidostina ei concinea, enon aucletiamo, che quan picí auevan:

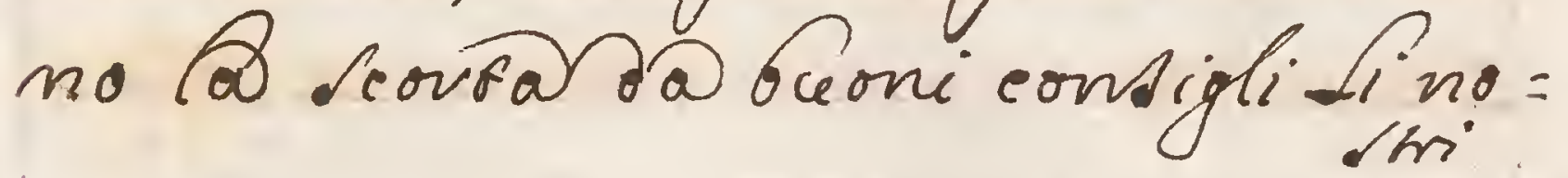


Atri pendienr; panro púu' megliover an: mo nell'esito, ef ad ogn'uno ébewno: Bo cio'ch nelle faude anvicho da quel = Racubifimi ingegni con gran giceditio compostosifinde che frelanto per so: tenero il grand incarco del hondo fu' neceptifato imporarne Taiubo a al

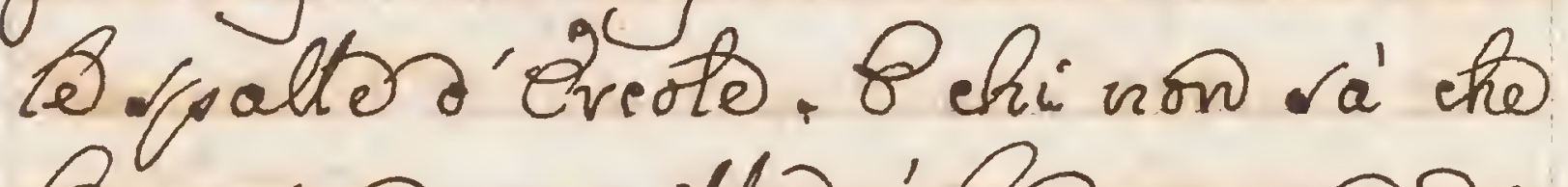

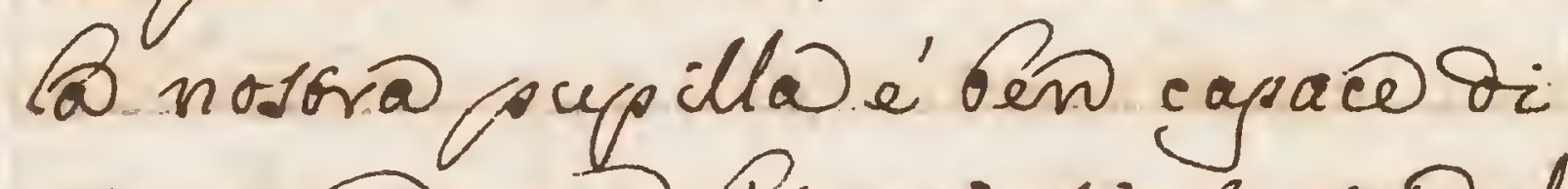
giv uagando pev Simmenti seaty del Mondo, mas nón gliésperm efso pevó

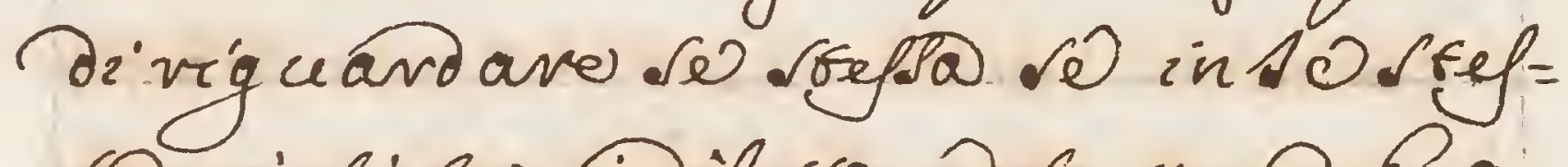

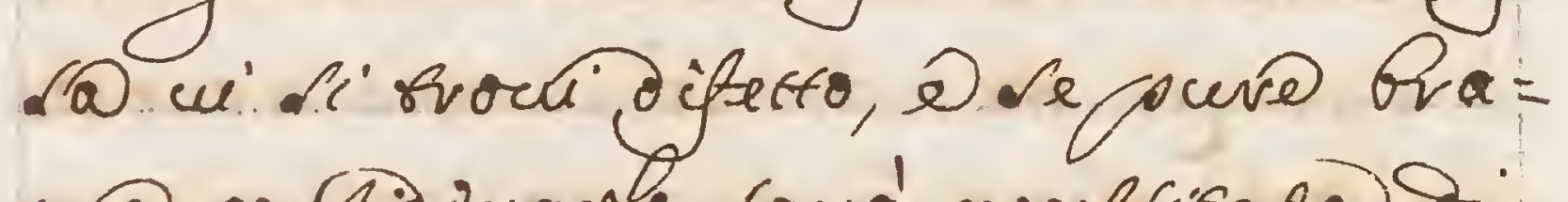
ma contidevarto savá neefesisatabr. mendicanno consiglio da uno fper hio, conta consulpa del quato sal uol = baspliforare con emento avne Difetsidoce man eó nakeva. Ma'? 
200

ingigno umano in hoggi orama.olo saggivarsi inoorno a chi sciéfecé, now à chificía dá. Taltra vaggione cié, non praticaba pevo'sote questo Relo, dowe il drencipo afcolfa con ecufsa'infinisarinalgenza chi che

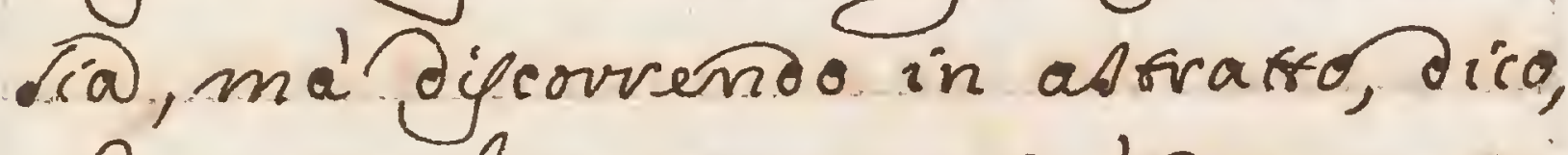
cho insimili conguienture e' ar necefsi: rás Tofeolfar outsi, ed a' guipar dell'bpo colartilouragni fiovo aneor che cilo per. suceficario dal rnedesimo quel po =

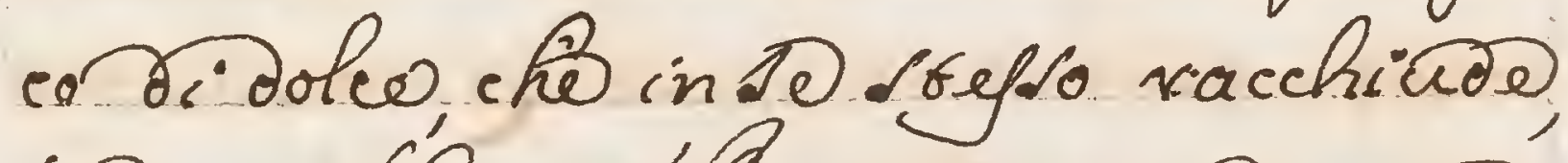

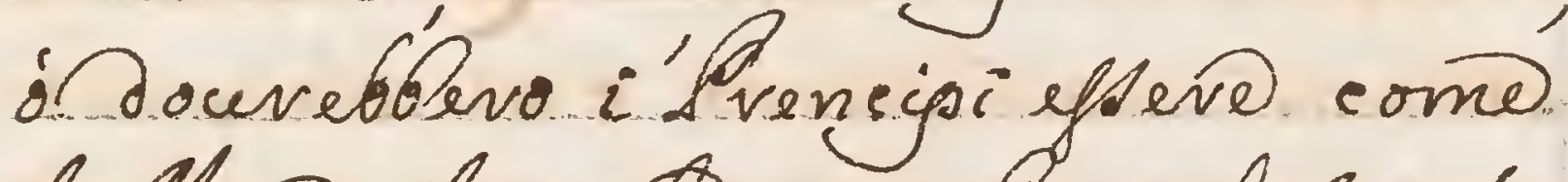
il Maro, it quate accoglio nel cio de: no, non jolo Pacquetre ifiumi Ria

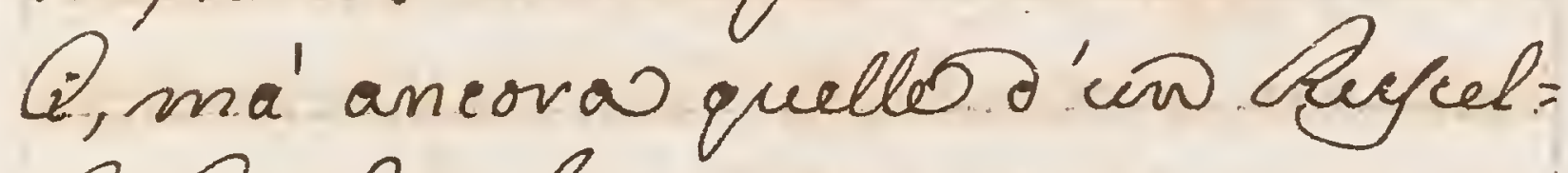
b, benchogli foontin ano men de gnioriburi, Dfiano un null riguars. 
Do allari Mi Maefón

Vipregorigratia, che hova cafíamo á cent uraro per un poco il mio Todello Senon per aloro of di: weroimtori sal'uni, iquali sosto $\partial_{i}=$

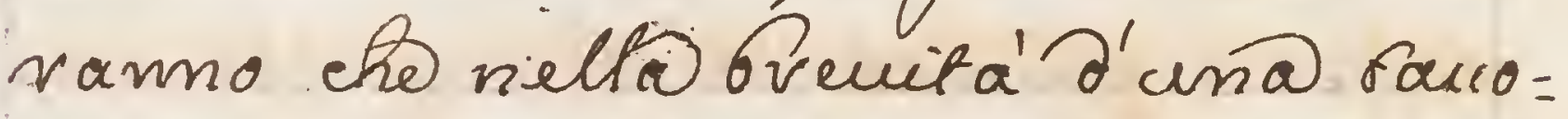
18. Nucede quabi empre T'operazione enzacuacillamento;machononcon $\therefore$ medesimi adsi cominapoi nel quande. Sempre condannables? ingegnero quaño ovijeal revroro da non efservo beño organizzato un mo dillo nell sw coarr; a' quellaguifa cer Pappunto cho de coes imperritio al vifefsore ciene mal' accomma = Data, ouevoposba frovi di proporsio: ne unaanna nillapiciolamachina D'un Qrgano, cervo cho netgrando 
$2 g$

molsiplearebbe Poncervo cell Armo = nia, et all'how Lavibbo renure alla pina chiopera, mentro krow hauen = Dow da principis viconoseiute ibjet = So, Droppicando insensibilm. Al inge= gno nel piceolo, nelli pagti maggiovi. Sel grande gli g'ageuolarebbe a pro: consine objonanza, ma quando dia con futro to circostan ben organizato. non porra' che cenfecto seguivne lêun = bo. Piganti aneor efsi nacquevo $\beta_{\vec{a}}$ orni e cure non regolacano lity afsi meñoñnell@guevilita', che divenu-

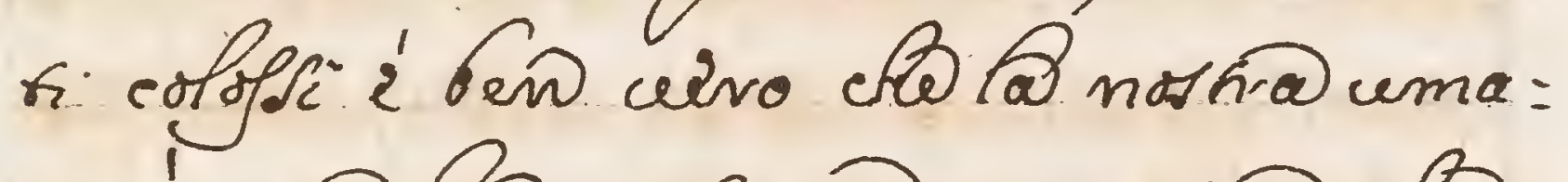
nitán now ha centeram magqione, che

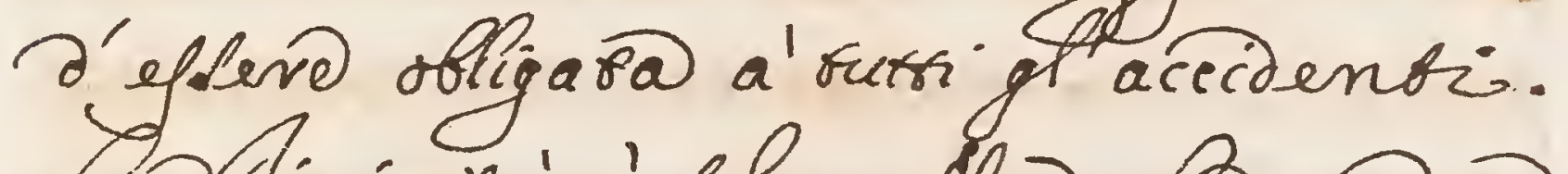

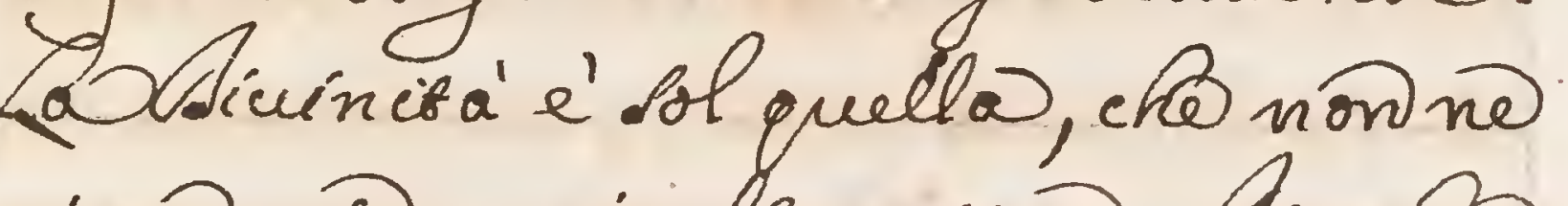

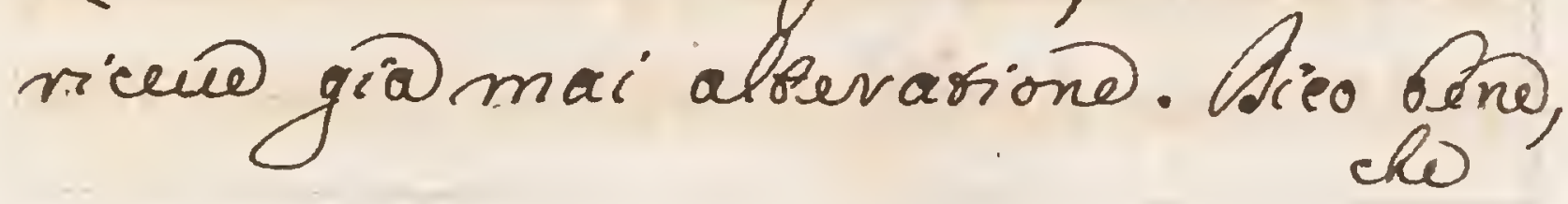


cho Bosulatiua puo ing annars: 2 che ral'hora non divifo biñ recondo Pintelligibile in astratto, e beno crepso rimand dusa se Pesperieña now 8 Sccourefso con Paicor della pratsi:

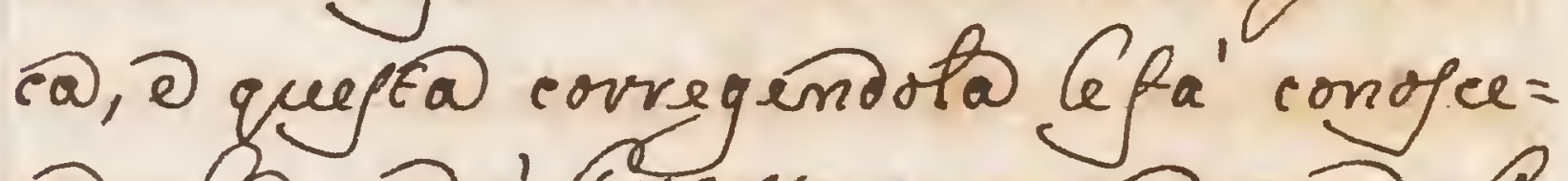

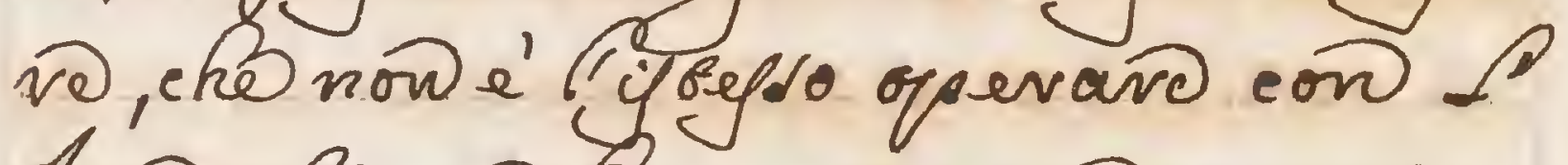
tô, che contômano, ma quaño A mano operida majora. Oncho che öers cio' cho con infelligenja ineo rradicifiles ella eveafso in piecolo con altrebantapenfettiono docuevebbo cov vifpondentoingrande. Sylogñ guar= Dansi a quelle operarione, che sal uolfo in un modello Laingano Fin: relletro, chor pel qrando sradycono Avagiono. Mir ato che i'rigigi, cho

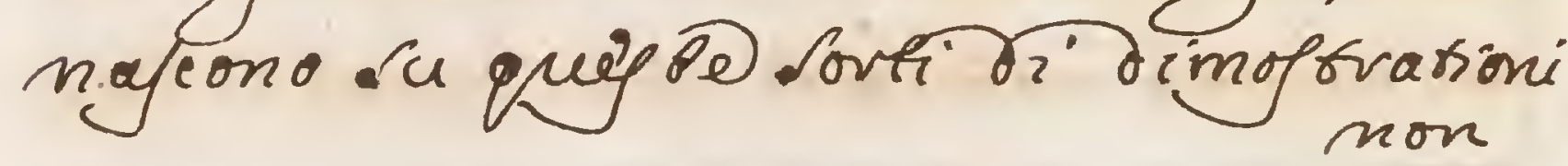


30

non dipogaino agitare alfrowe the nel Iribunater dicena veato operazione. Sorvebs ben dirmi bal'uno, che forsi: all'horo io corvei vimanere dal mio. modello deluso: Rifpondo, cho cio'é incerso, ma altrest' e' cervo, cho fin qui is habro accordato iders'co'ifar: ai è quanro ho designaro vell'trea, altret anro ho vosto in atto, es con que = so modello ho pueteso rappovesentañ a'gl'ocehi himolsi cio' cho moth - con il parlaro pretendeuano hi cersua:

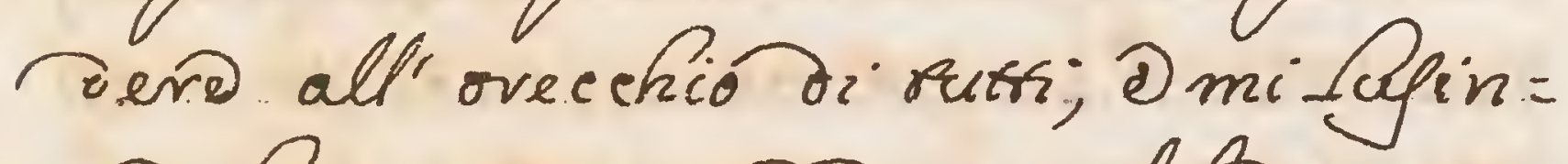
90 Papepvouatioñ di qualch inten : Sente Jjapeafsionaro, il quate vieno: - centolo mi centuabe, che non. aveb= 10 forseviufito inferete nell'operoma' con fucto ico' non fava' mai insup enfirmi 
quel debote che cosi beñe vieon oseo in mo segso, d cosi non pretendo or dave ad intendere a'uevreno ch"io Grami $\partial_{i}$ acquiffare un evedito aperrone al cappis ato

A caqione, che què apprep: So is hauro' to vappres in arret qual= che demstruatione geometrianon la =

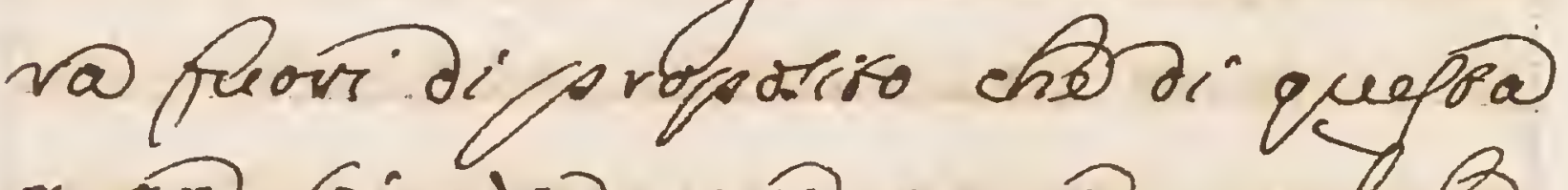
guan cienja cuno corga qualck raggio, D.eña inotovarmi,nellague.

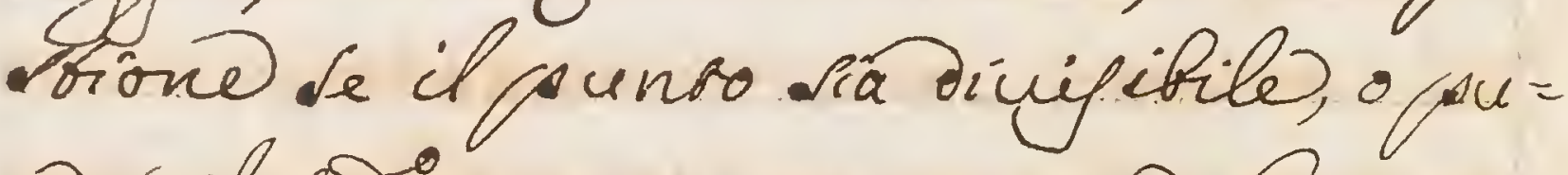

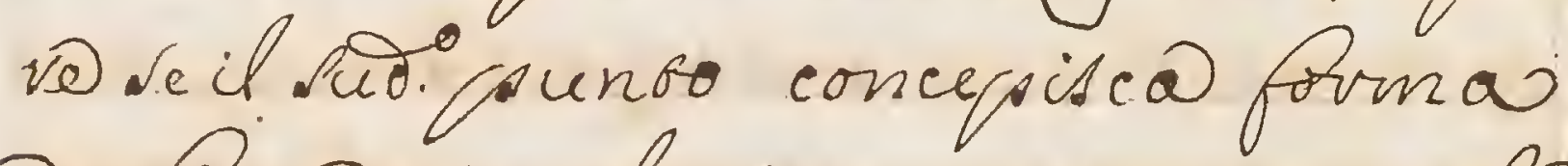
dilinea se egli fias una corbe, th now é, se rio prineipio dello Linea -non Sa_inea, comenell furtome= ri@ilnumevo e'prinejoio Del nume= vo, Onone'numevo, come il cuono nella 
31

nello mudica e' principio ach Lcono, - non é accono, Rabanbo wel fenge.

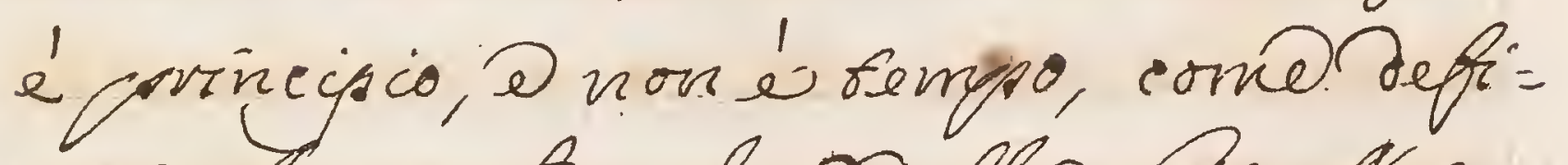
nifee Aritso fite nel orella fitic. Me ram: soco enovavi àtrui sela likea.sia. quantivá confinucto, Sela cepenficié vimang@.ennā profonditá, oguna. e

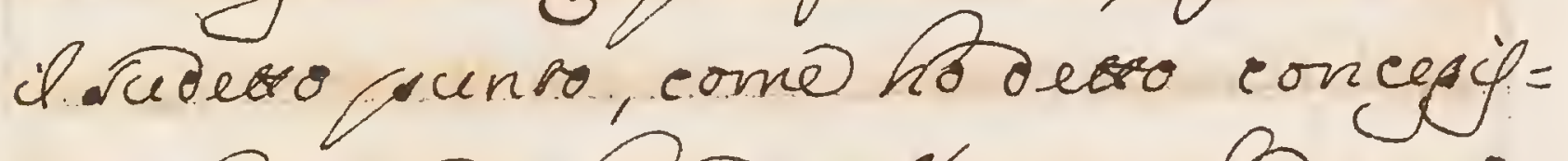
¿arotrionirinea. Ma giach mi

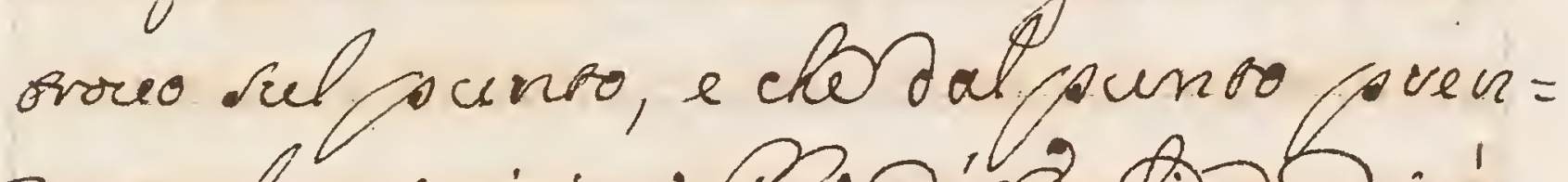

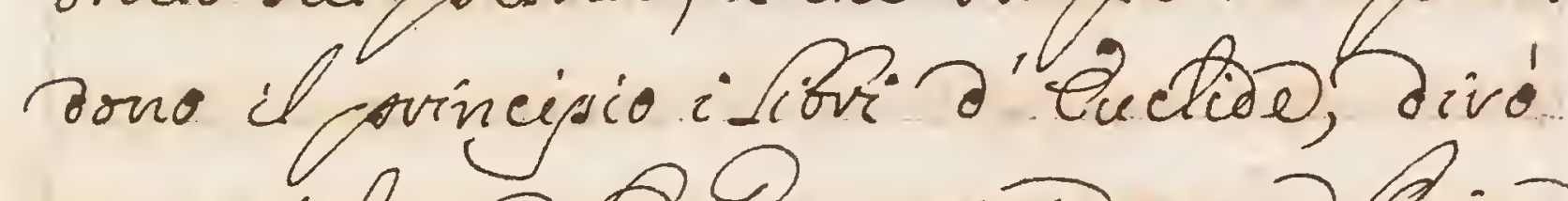
cer esalsave Poomerio come Reina

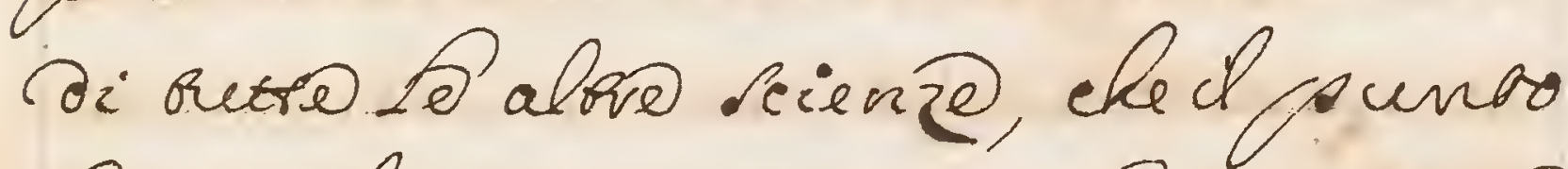

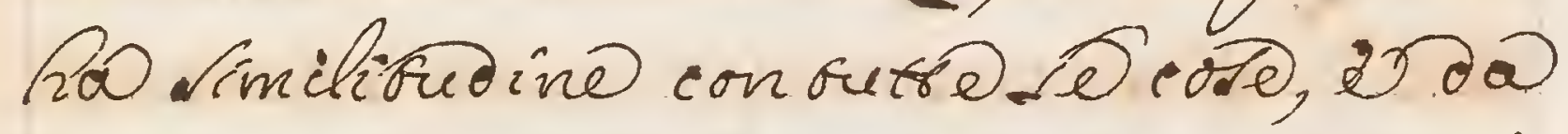
rab anticiena anchono accarderá?

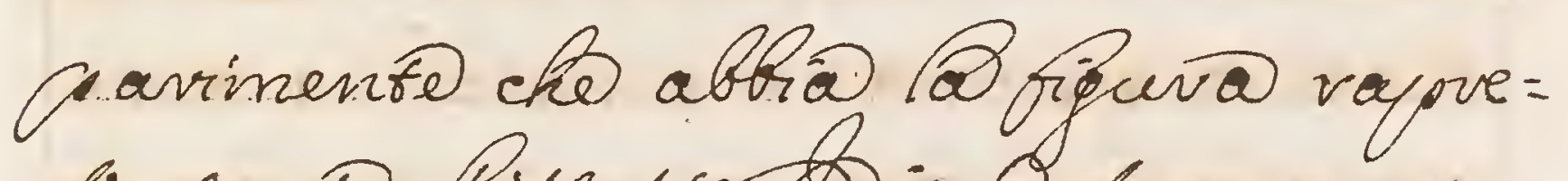
intanto Pisesso toic, P. cuppongo. che a' quisso viflefso conil paver be $a=$
ciens:- 
pienti all' Inniporenza Aciuina ghis atonbli a vehe questo nomo di pounto ilqualo sivincieno fio quelli Pans' al =

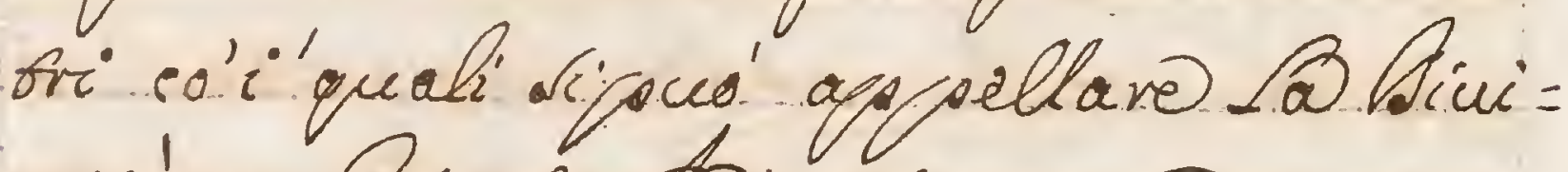

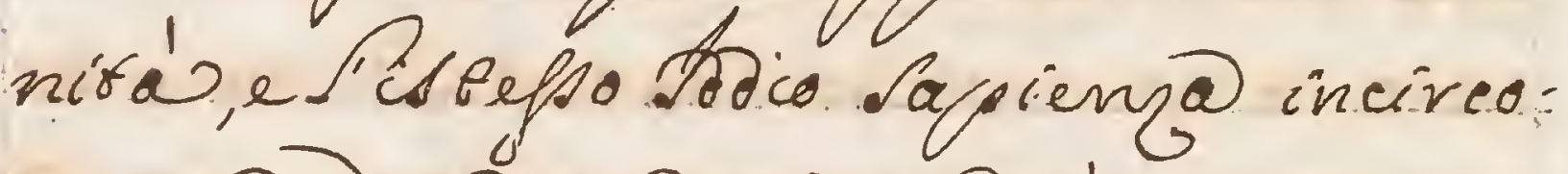
seviob docionon giungo a penerañ Cuman intendero gefi ch'é caubori

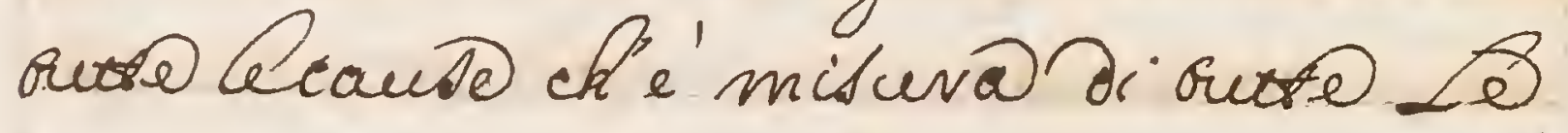

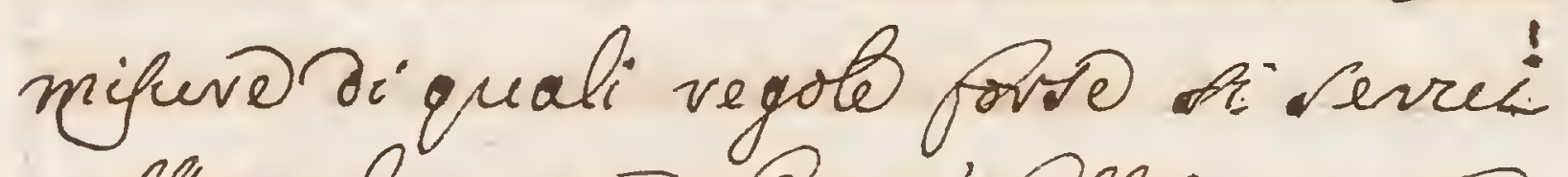
nell avchisewañofocie Blla, eruaga

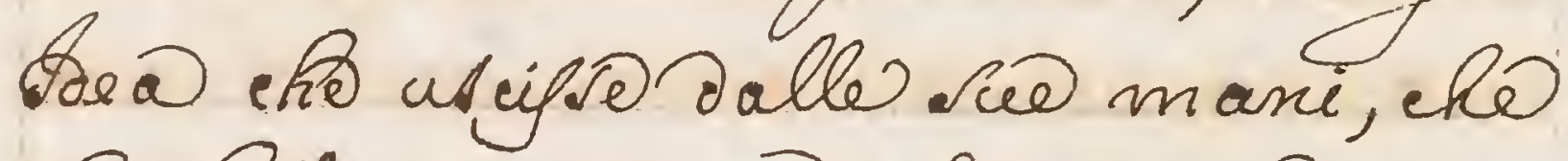
fu Promo, crediand che per bene or = ganizizanto now adopero cheta geome= fria, of qual scieno a fa conofinto -uelatamis: fistegso veabove, er e'quel=

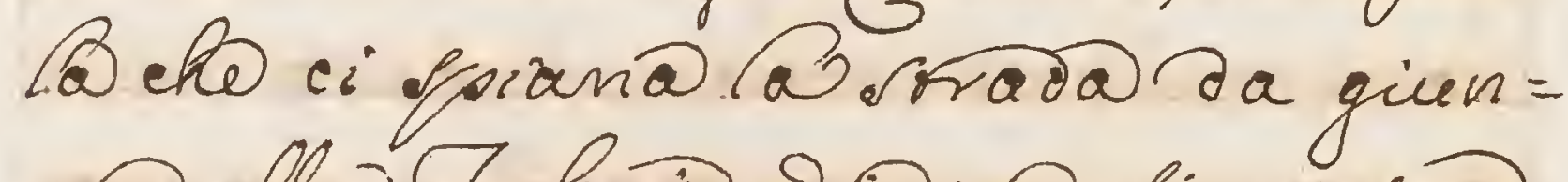
geve alla Beologia, hrored ariua at dicpoi alle speculationi Aivino co- 
32

mo afferma Nieoló a fusa ardinalo nellor rua rpero invisolaba= Omple: mentop Dhiofogiew fig wevarro in comple= minoid mathemaricis = uestala

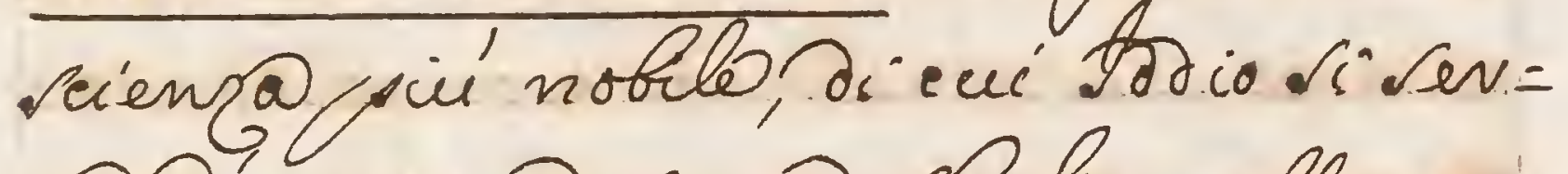

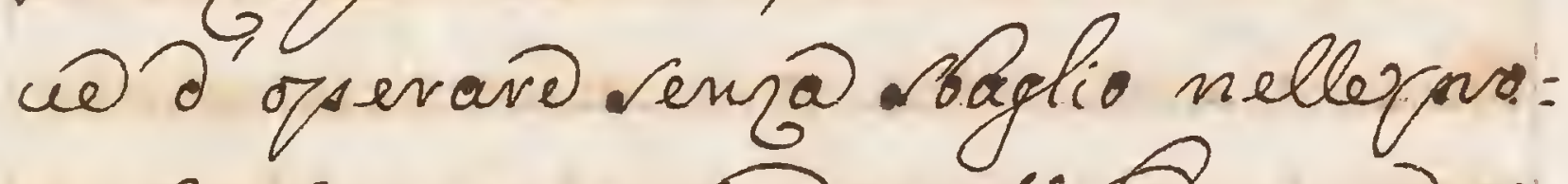
consioni compartendo codi Os pena co = moil cruemio

Inorodurro' in questo difeorto unpendievo di Rinabo. des Grbeffian= ciber non infervio a' curreno nell' efore con bion firofofo, epartic olarmento nel torotinadel moro celeberrimo mare= matice, e chinow erroeva' che egli beno. Selso non sidia vifori. Aemoeribo, ef Epicuro, iquali hamo fonoto il foro. -vederve nell'egplicasiond de i' principy. Del eorpo nafervate conla dotrina je 
gl' aromi, ansi non hamno vicon os cuevo. alwo en tie the quessi viporpand il fut = to a'quelli corysuseoli, di cui noviman: gono composti; come che pavin se fijgor Fio eglipur anch ha accommorata

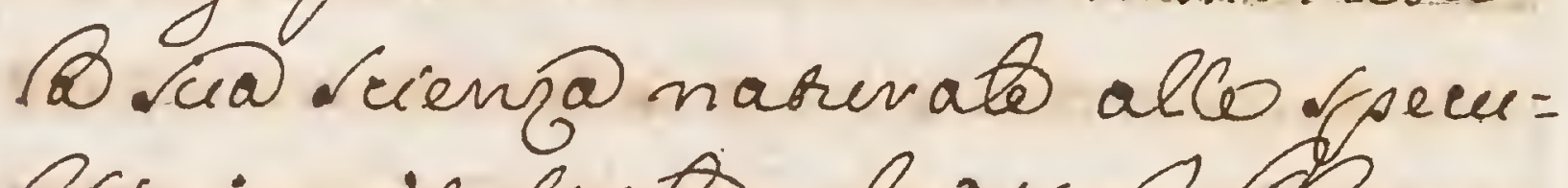
afioni misafisiche, al wisti liaroniei yfends eccellenrinella Joologia nabu = vato, o nelle cagioni uniciensali, hanno

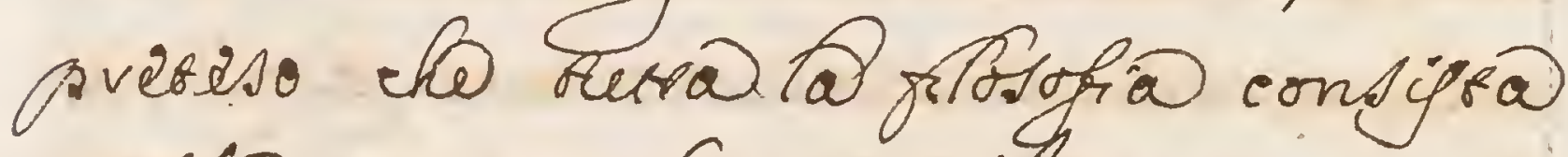

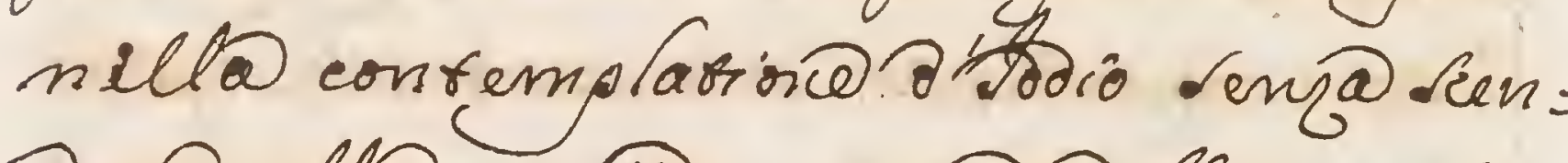
dere allo contidevazione delle caule leconde, m'́ Idoniarita aro ares ha it Gutro vidaso alli grincigij di Teomenta ma dicome non agpansine al mí ribunate, come Gudico incompesente illefinivne@ire,cosine. ajcio all'in: gegni bi maggior el euatione, ec invelligen = 
33

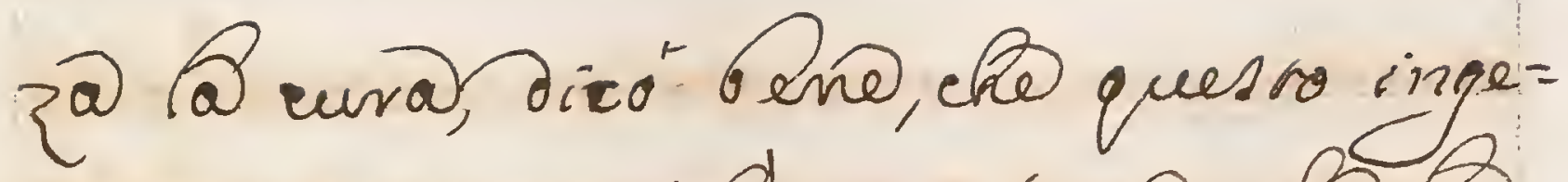
gno feregrino cifa conoservo, che $A$ Leomeria cifa sradadopencuevive ad ogni Sienja, eo fippoevare seviuen: Do a Jefsalo acioglio inceña Letreña - consiglió a appuenacre $\$$ Peome: siabicendo = a cognofundaleometri= am multum kudy athibero, non Solum uitam Ram illuswino, Sed esiam animam acuriovem, es clarsove vides

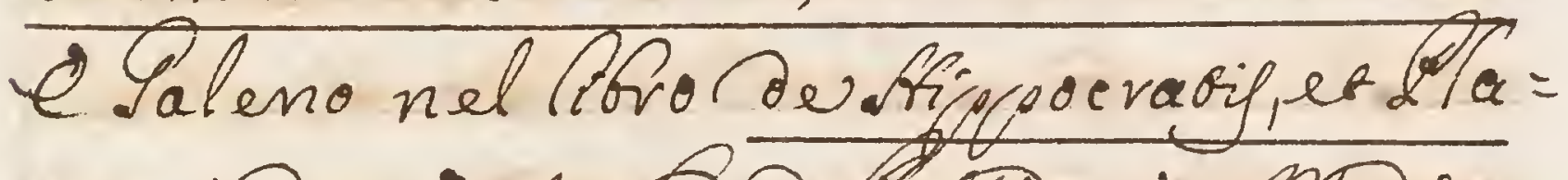

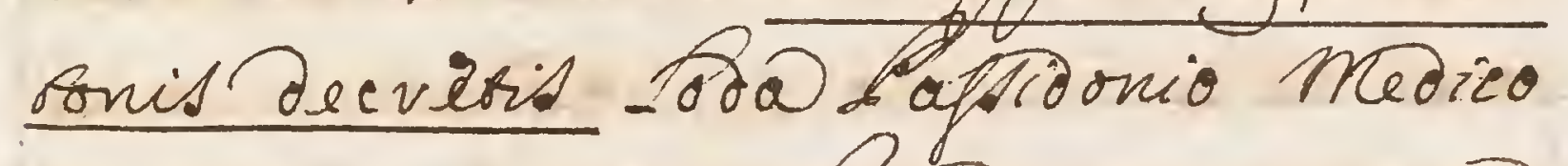

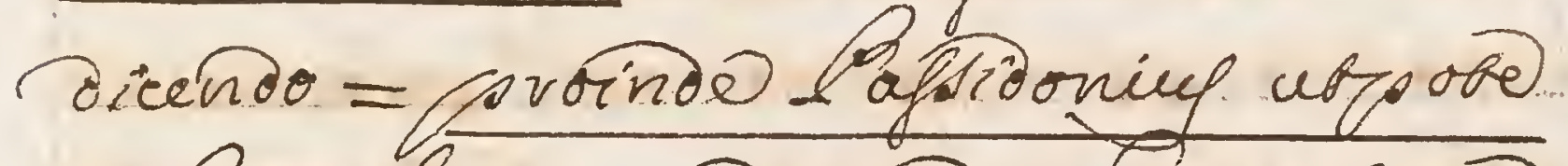

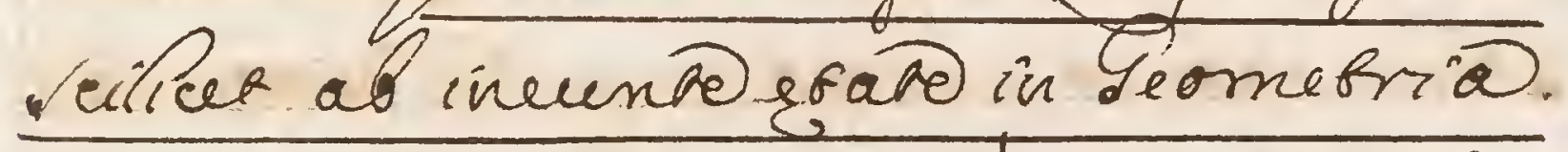
cuevabut, magisque quam ceferr froi:

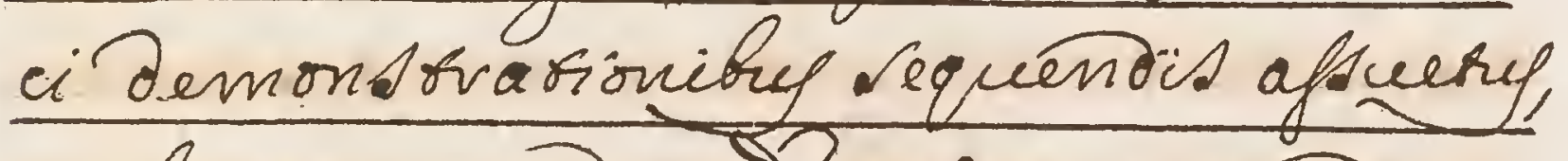
errefuit contio euidenrer catensio cer =

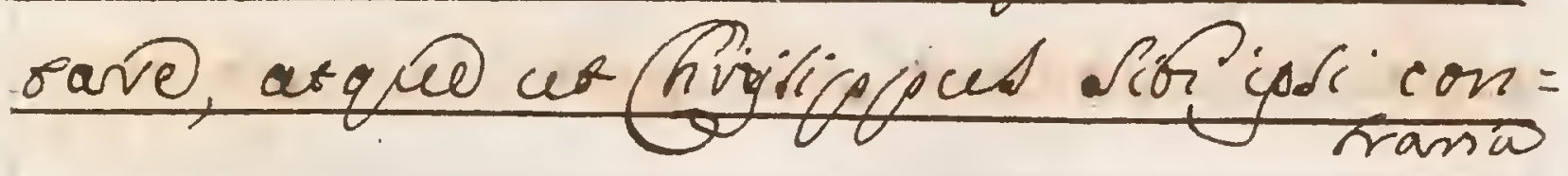




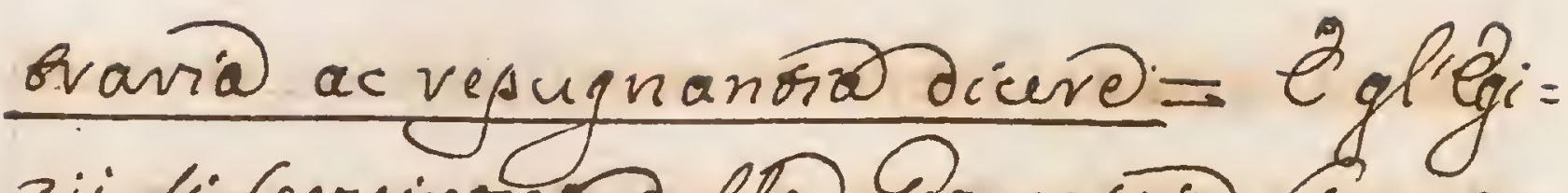

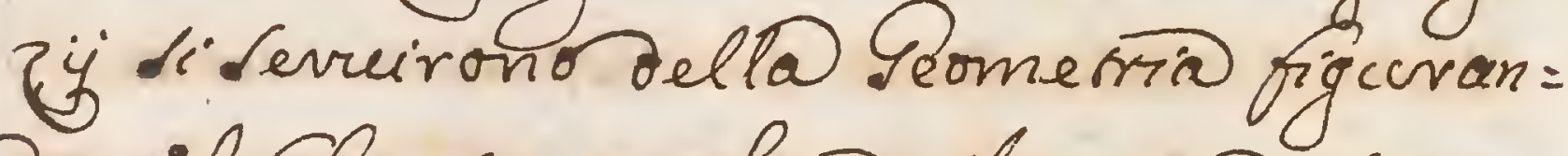
ao nel ubo triangolaro il rumo supe=

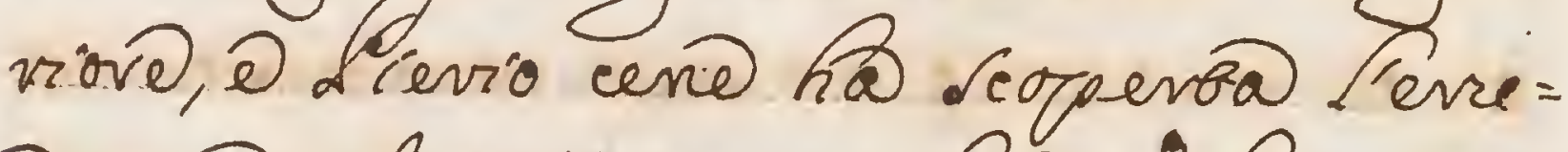
dixione al 3.8 de geroglif. 2 कि nosina Religione nel triang olo equilateve coes = ferramies esprime-con Cequalifá belles parsi non virrouarsi Jyevepanza fra?

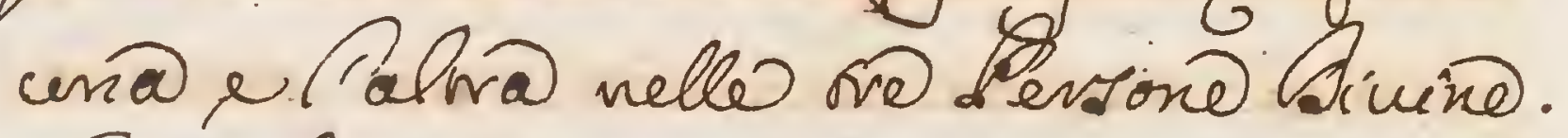

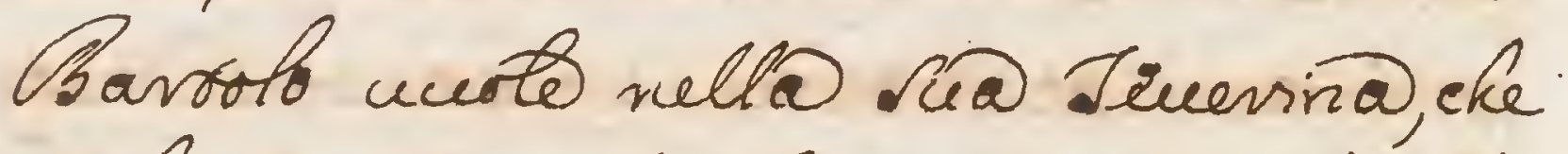
inluro. cicú che necefaria. Non é

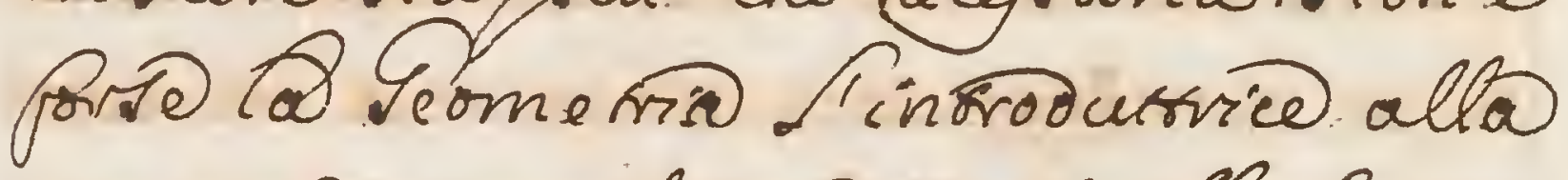
crvatriaseculaticadoll'Algebra, er Hinucabuta, uolgarm en betra ta vegolorall' anie magña, dello quale. aptevifcono cho nefopse il curimo inuen = sor Moje frabo, es geomitricaminte

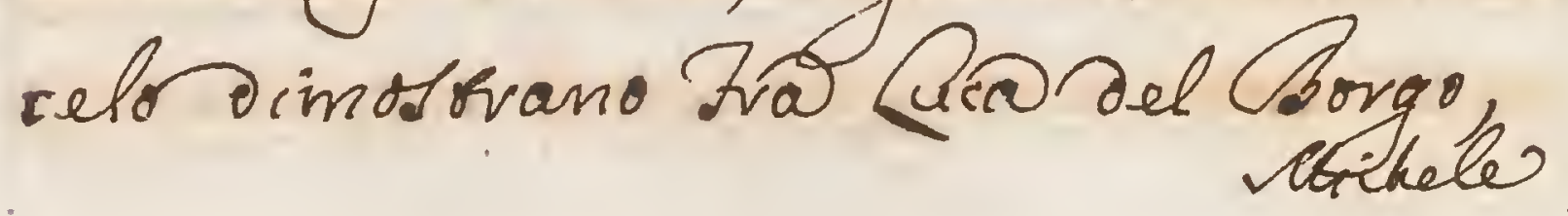


34

Michele. fifellio et almi bell ingegni.

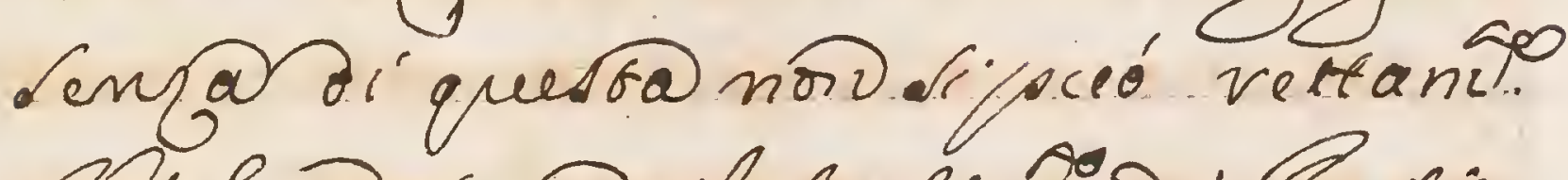

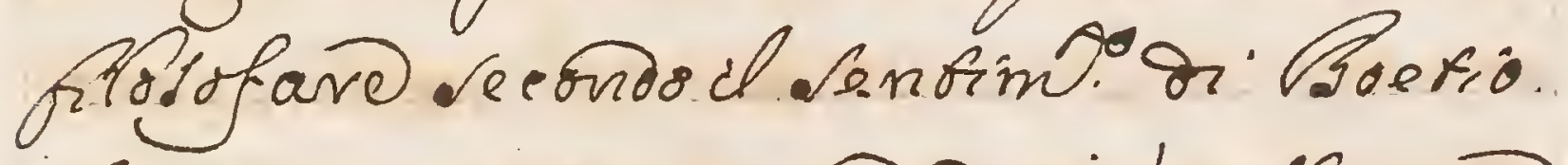
Luivino, etingroias icio' affermo che anerche folso pal'uno un gran

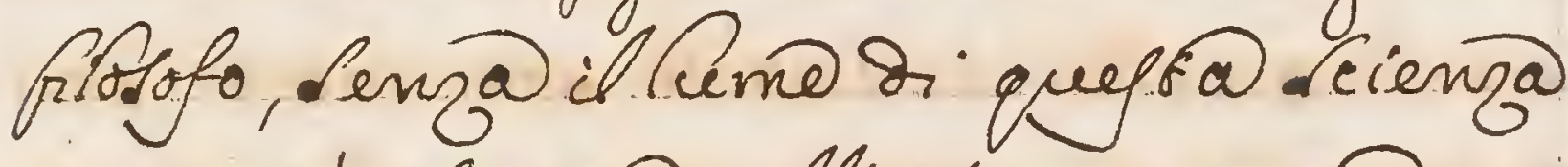
vimarra' Semporo all oscuro quaño ui.

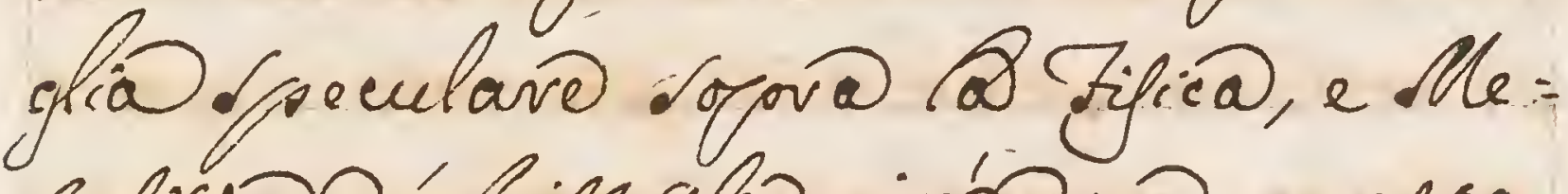

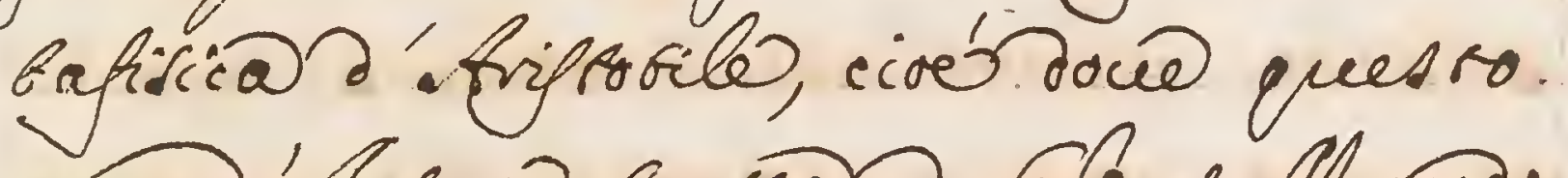

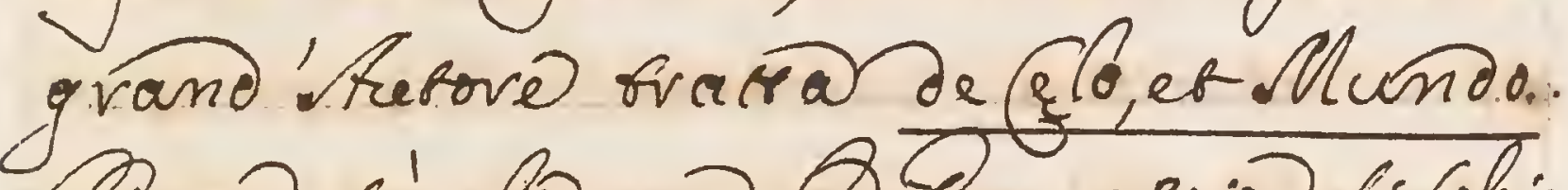

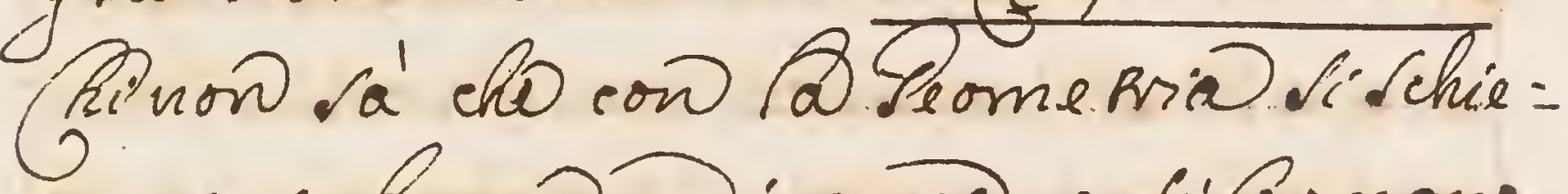
vano in buon@ordinaña, ediformano Lefiguvo ben proportionate cosi be gl:

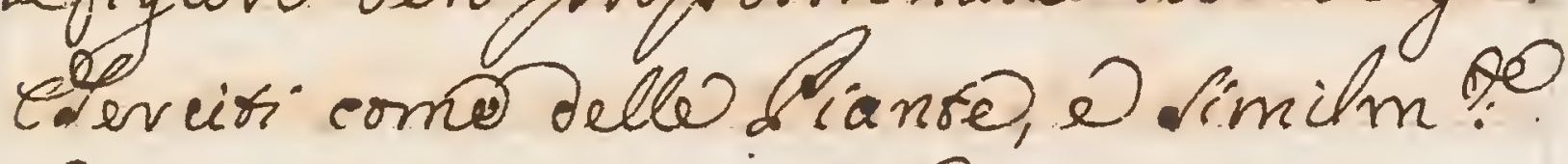
Bforfificasoni.enja10riondenume= ri, dellemifurder senja lellovo prropor: sioni, chejifedo faveboerro a' coloro cho rimangono civeondar dallo medesime? 
Aquali cetosarebbIIfnima@bixfura, et a' qualia afi dareboe quasi.

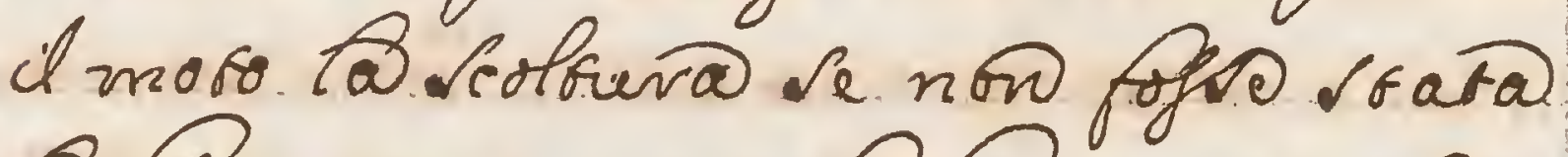
18. Poneria; anj to Pomeria ben condora nello faree di Fria, e fraf: riteto quantinell' fube statuaria ta jucepoli fian fatro dicenino Magtivi. Il nomed' Afrchimed Sar ebbe vimagto - cepolto nell oflicione come Lempro Se n'añiarás immorfalms. g forrso coersefsensi opposto con il pofeño $\theta_{2}$ : quej@.cienzallimpero h. Mlarro Marcello onsoto Romano, \&qualerípor= pó maggion seonfitia dalla Itomiria mecarico 'trekimed, che non have= rebbe vicuucto da cen poderoto confra = - tro diun eseveiro nemieo. Racconsa Acesareo, che questo projofo evalinna
moraro 
35

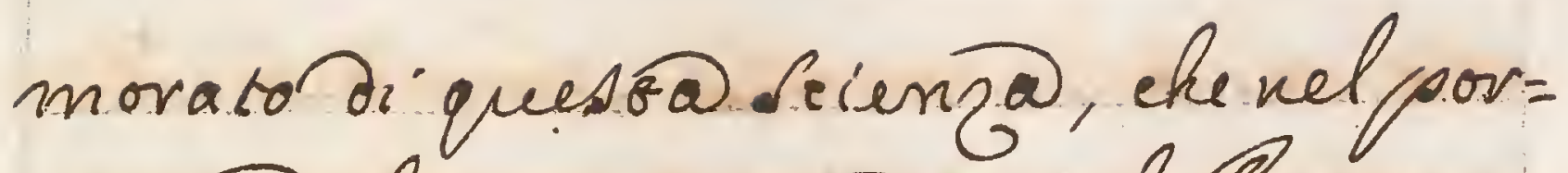
- cartidal froprio budio nel Bagno, es it suo congo effendo gia unfo rutro affrat = so in questo oudi, sevecñoli isto Sivile, etil corpo di carpo, cu fivace. al h. Souror belle Lineo form ano ou fi: gure geomericho. Pchinon 10 , che $B$ figuvero Jeoremi, wa problemi geo = merrié evano a chíne per en rrave. nell'berademias. Jarene. On qual

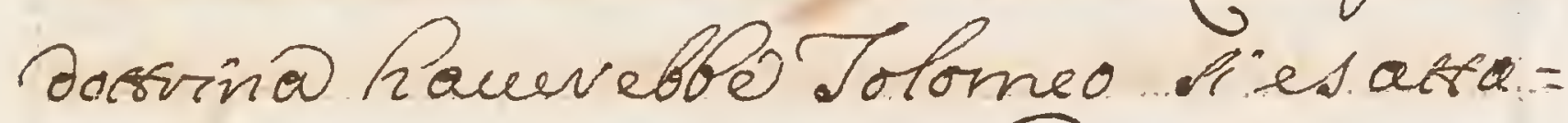
mina oracuro della Segrafia, for = - con@10vaticarauronongiió

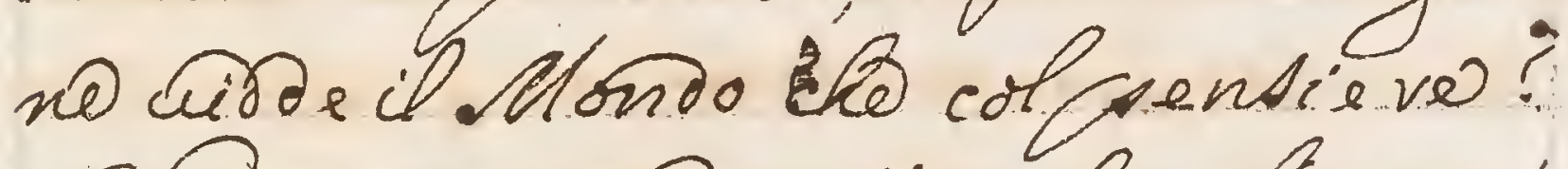
\$Comeriafu'quella che gl apori \$. orada, ondefori eon il numevo. conse mifure, conta proportione, e

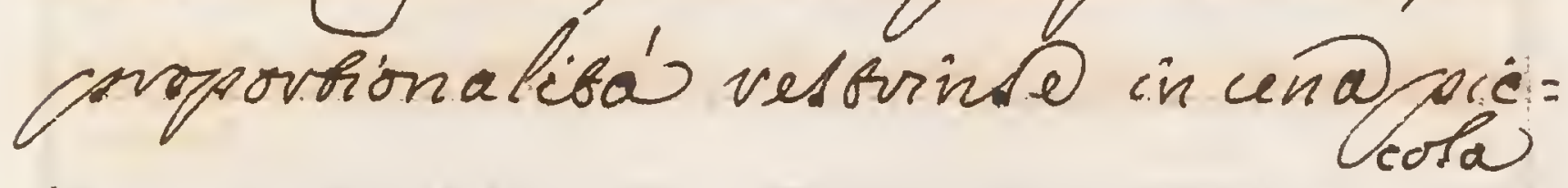


colacandimmenti fpariallovi:

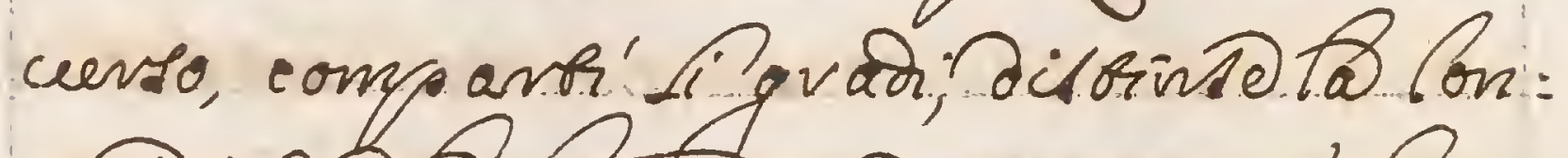
grivine,01ativine, et afsennó fipvo: priácir allo frouincie, alle crás efino. ad ani beñ piceolo Villagio, comopse-

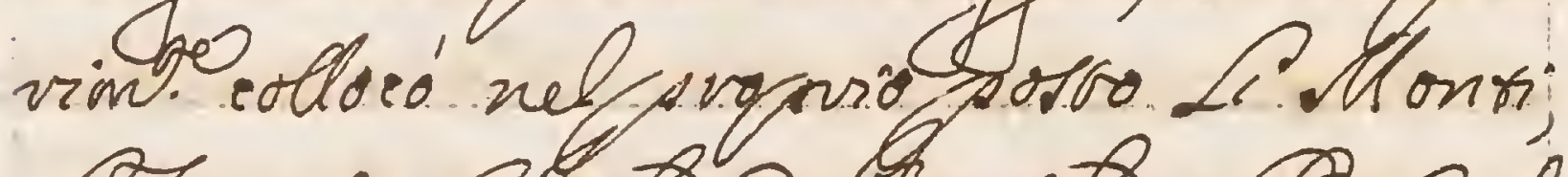

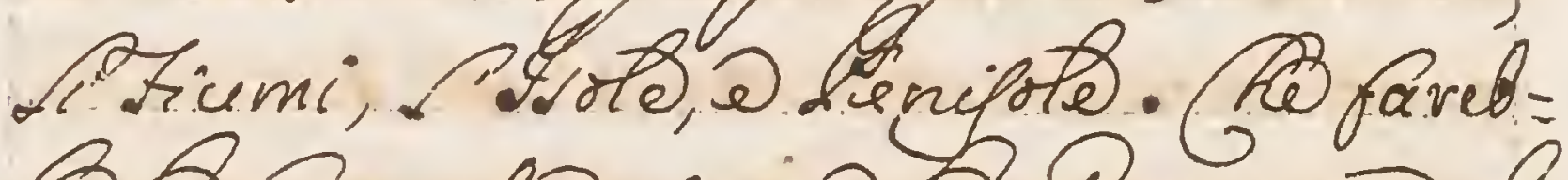
b10rourafia serija to Someria, che glismivuracincicior qualticio

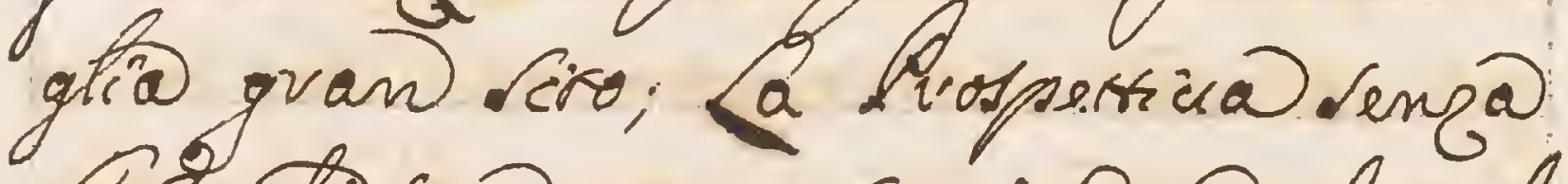

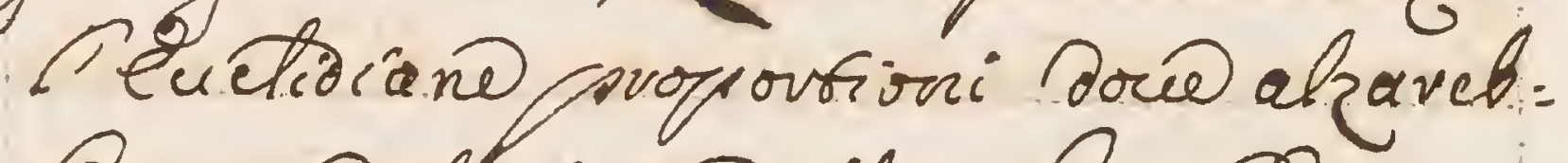

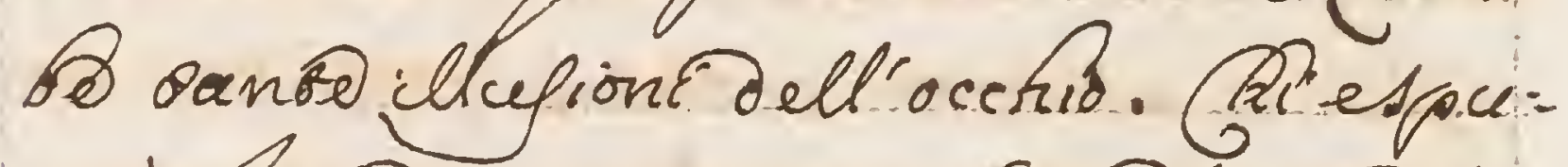

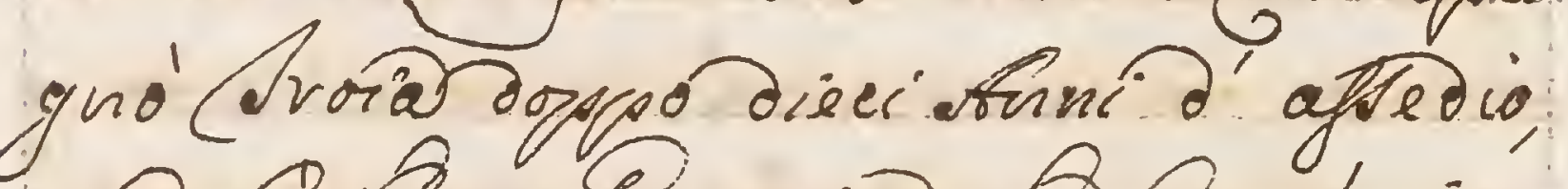
non fi Gpeo Someria, che fromo ino un@Machinaunfauallo, al qualoin

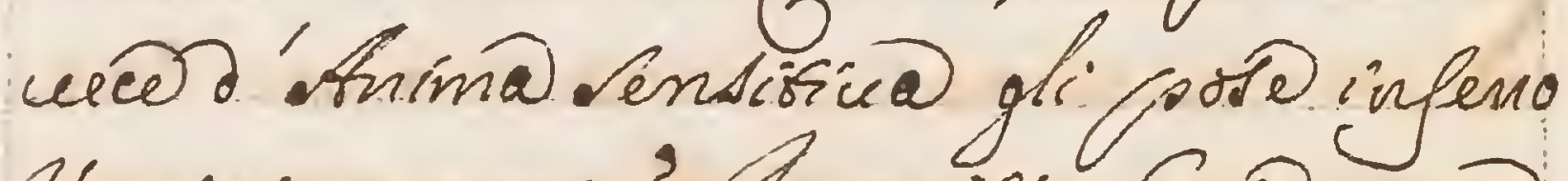
Vomine avmati? A a quel bo fonto, cond afferin@Solomeonell. Amagesso,es 
36

- nellesculauto no scarent Pfrolo: gia qudiciaria, a qualo ofperifo infallí

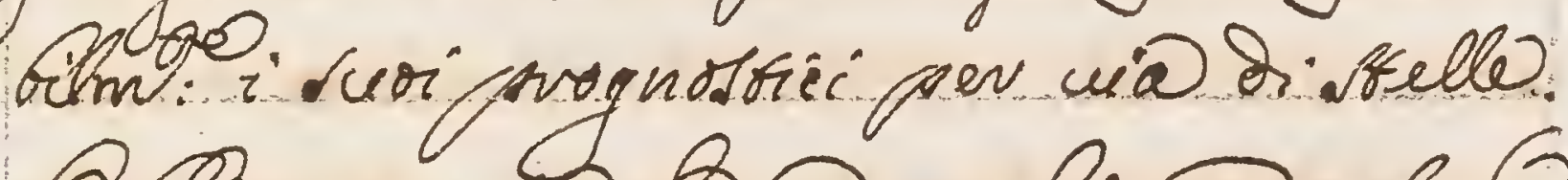

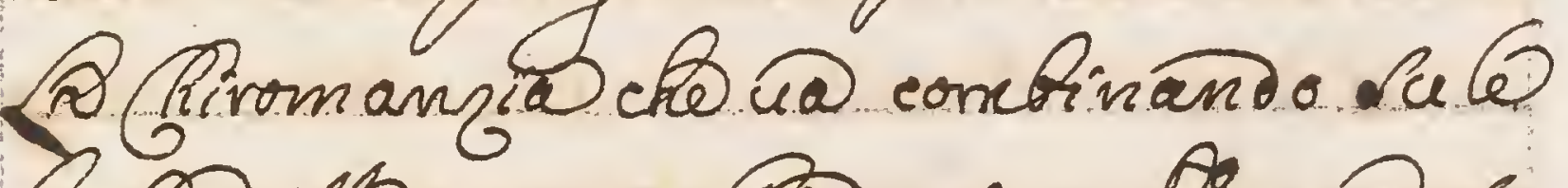

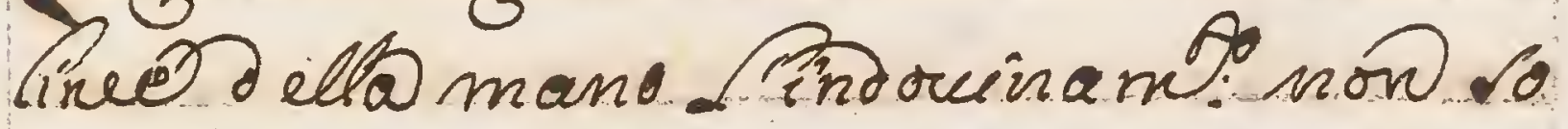

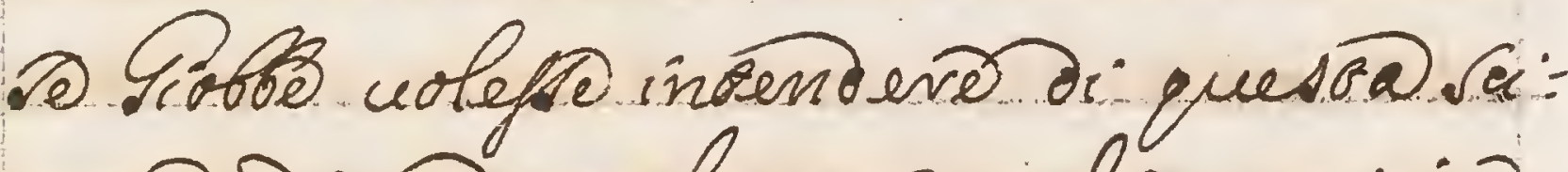

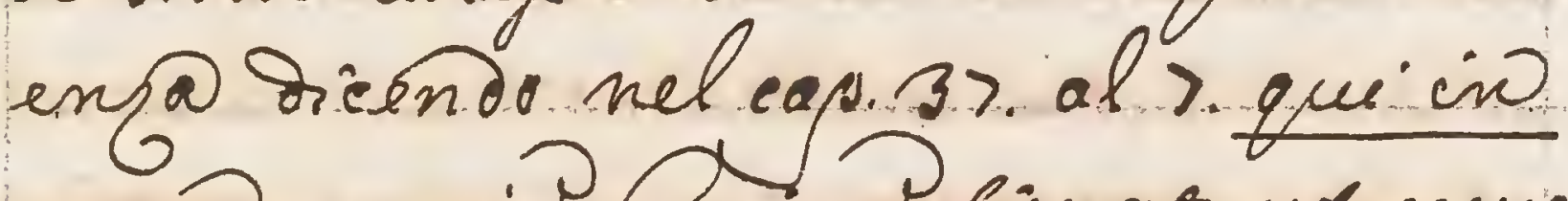
manw onnie hominu Peinat, ut nove =

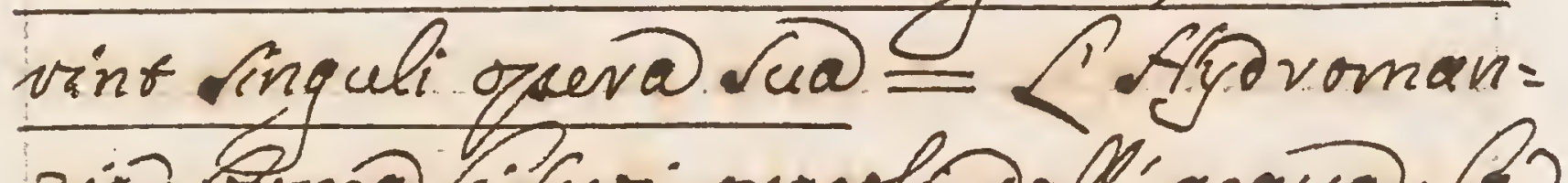
jiafoumaricur ovarol dalliacqua; \&

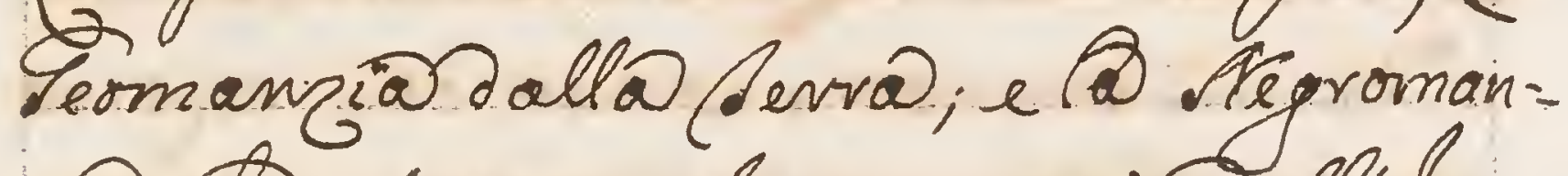
ria hor soto con il commercio dell In.

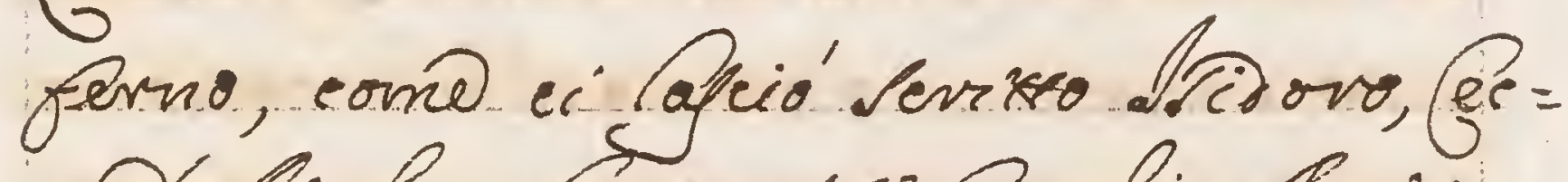

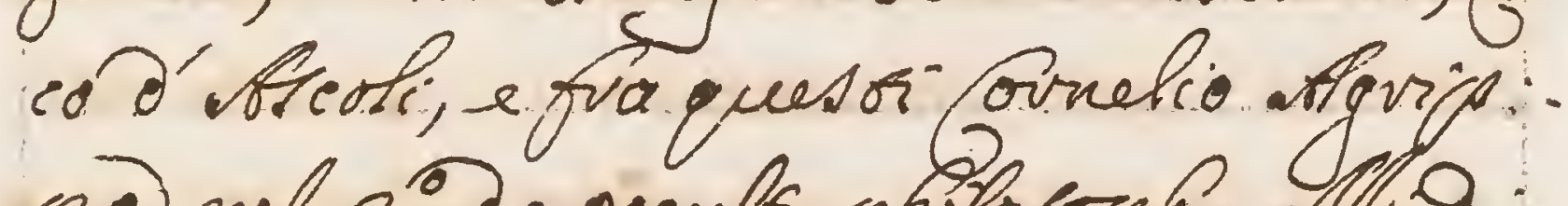

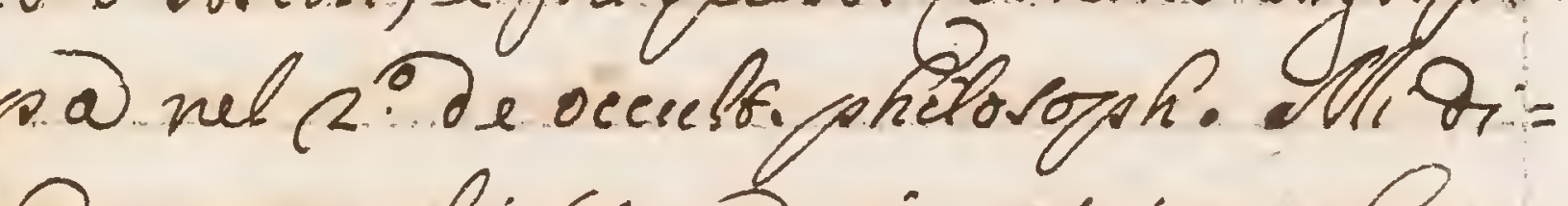
¿acon quali fienjo viconosciamo lama =

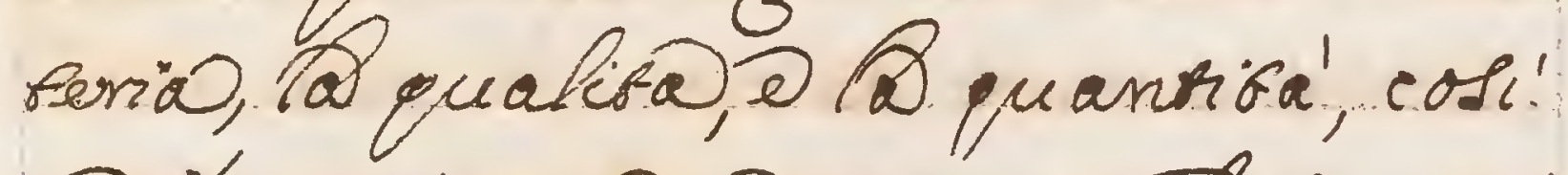

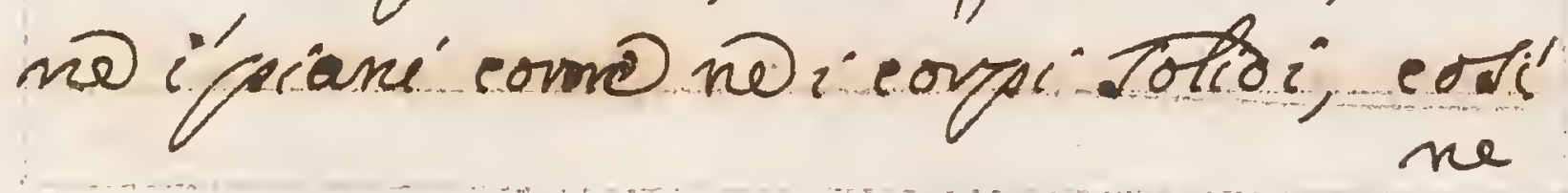


me i'vegolari como nell' irreg olavi, cécés Sivamidi, Sirme, ferve Gini, Gindvi,

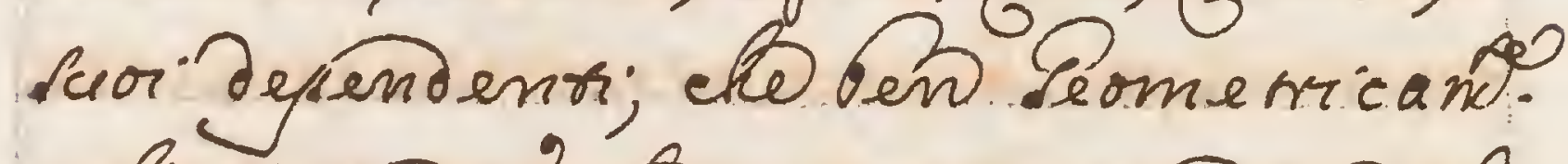
celinsegno euctio prinegiando dal imo lino at deumo yuna de sur

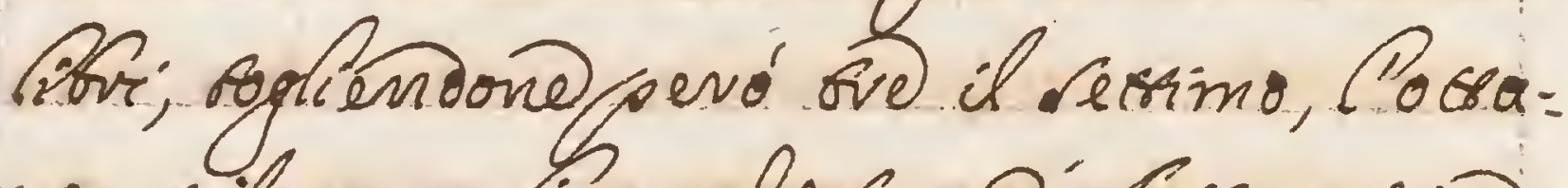
uo, es il nono, ti quali ono á friomerica, cril quinoo e' commune, che oraranio Dipuppovioni, queble Saccommod ano co= "one al numevo, cond all nifura,

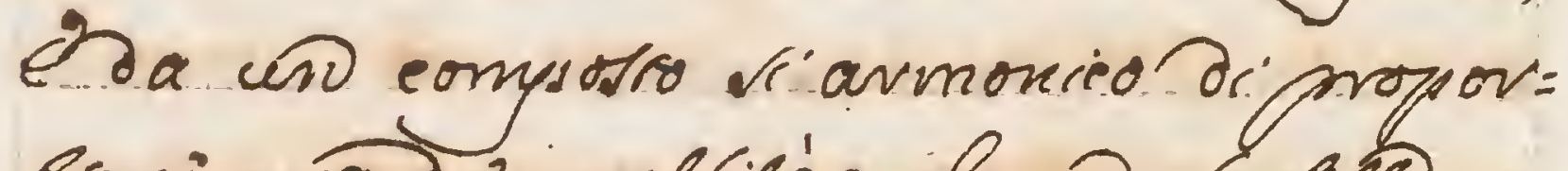

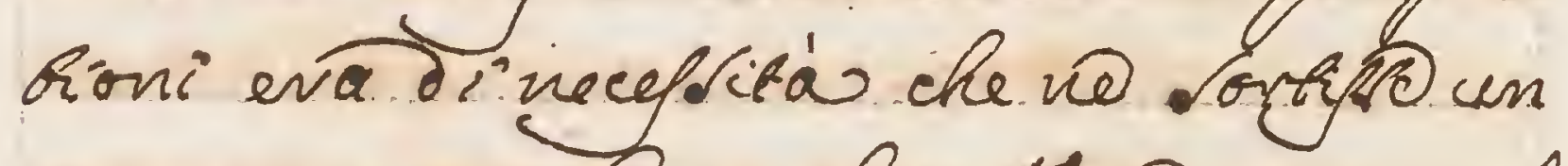

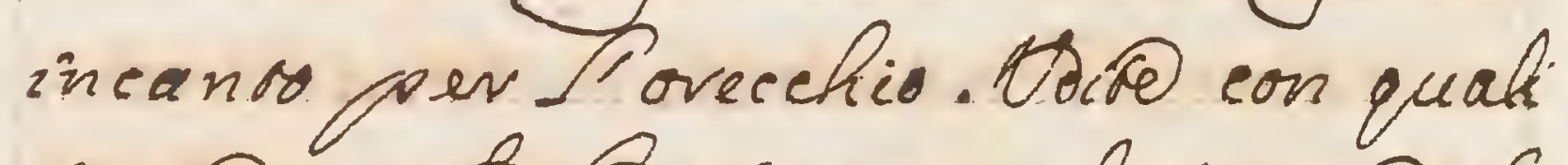
Low divegote has apuro nel cuono del:

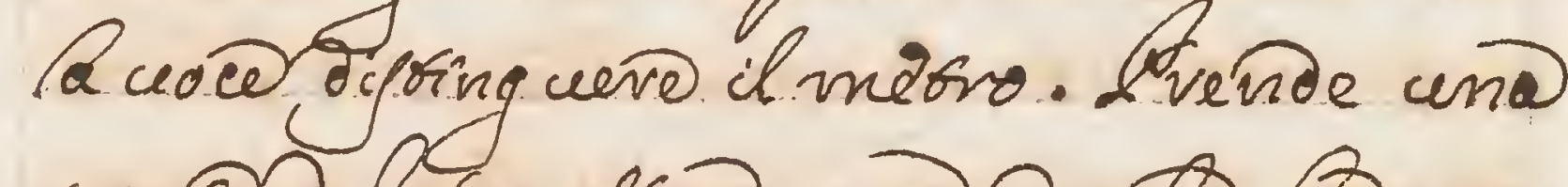

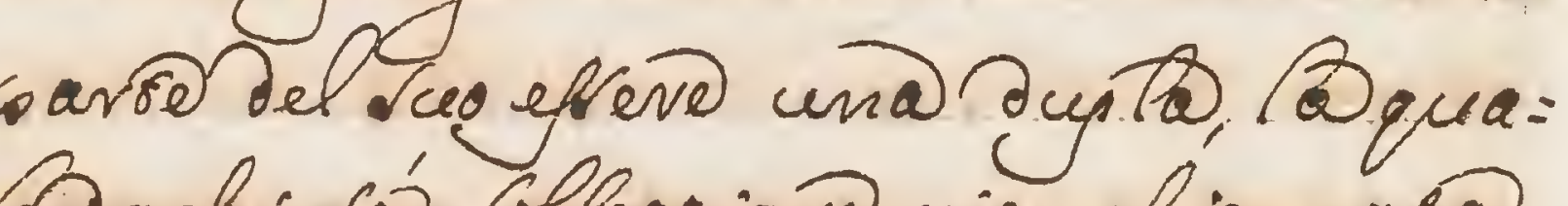

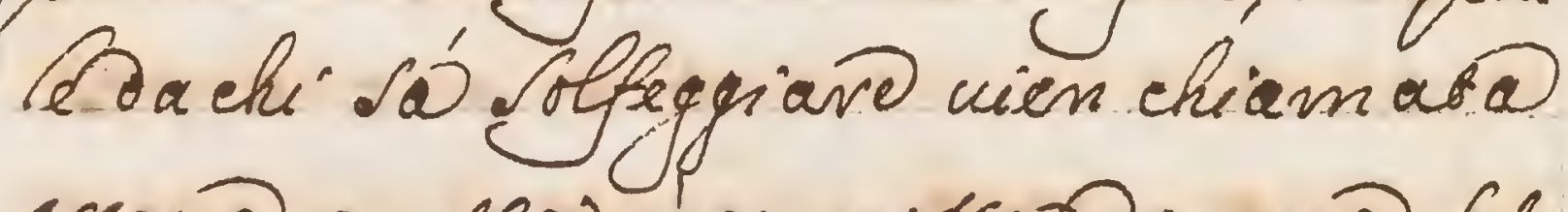
crand,quepa e compodrata und. fl= 
37

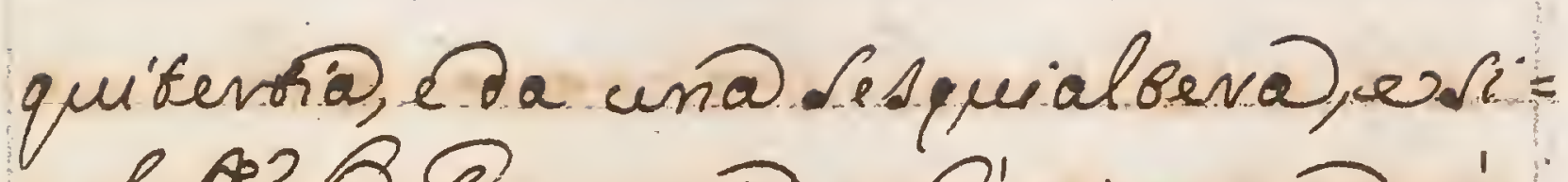
milmo@Siomerracifágiungere à conorevo che b. Spuitenta compofia adô roni, ta an Smitano min ove,

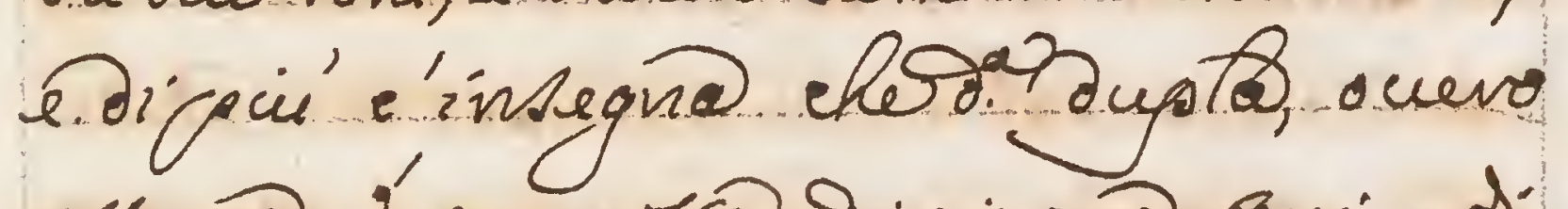

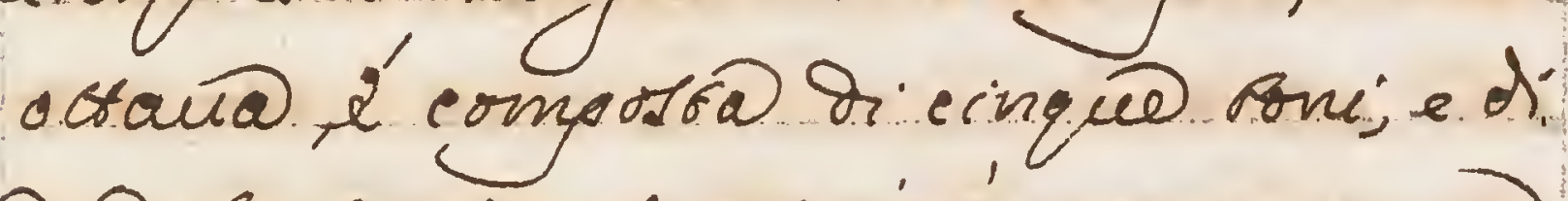
acel Semitoni minori ció meno una

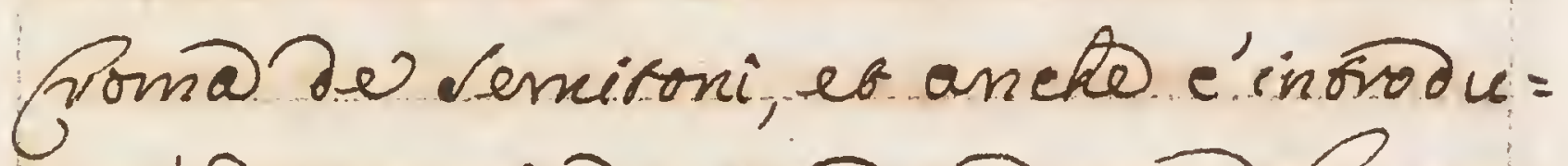

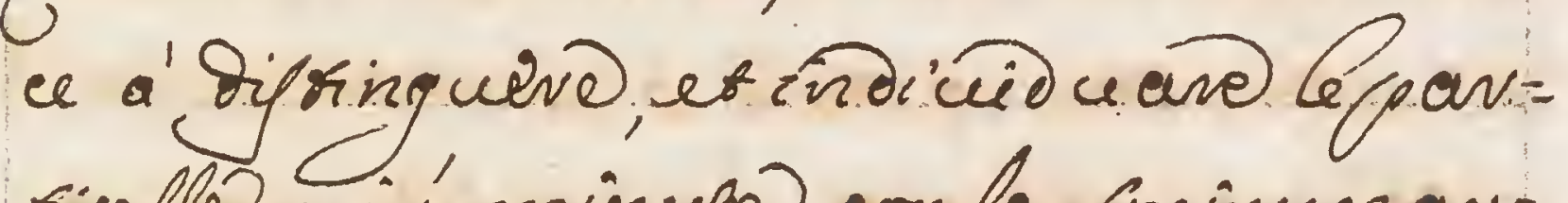
sicello cpié minute conlo iminuzzar -

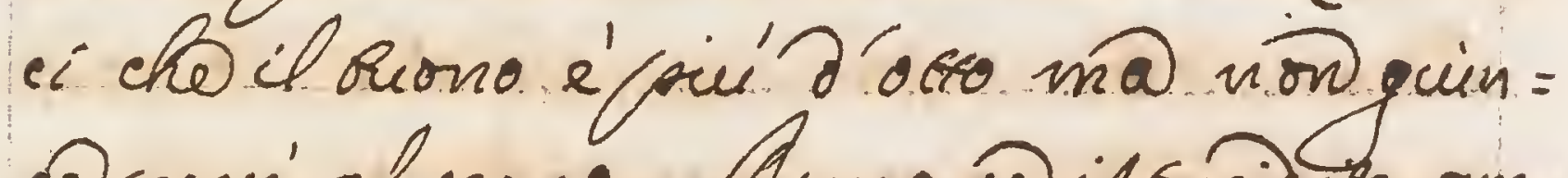
goperó al nono. Aunqui isrupacto am -

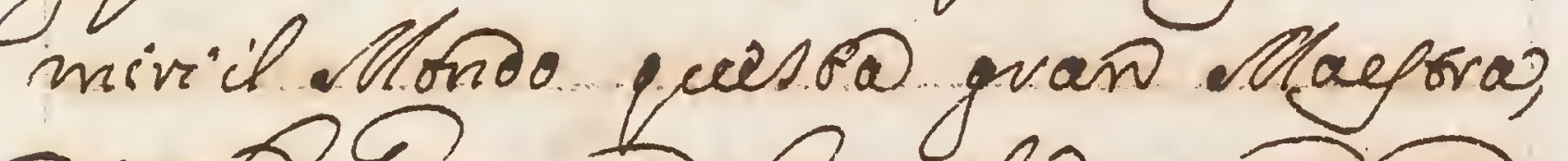
Diso Promeria, aquale civendead= Dotrinati, ch' e'inzogsibilo a Sicidere

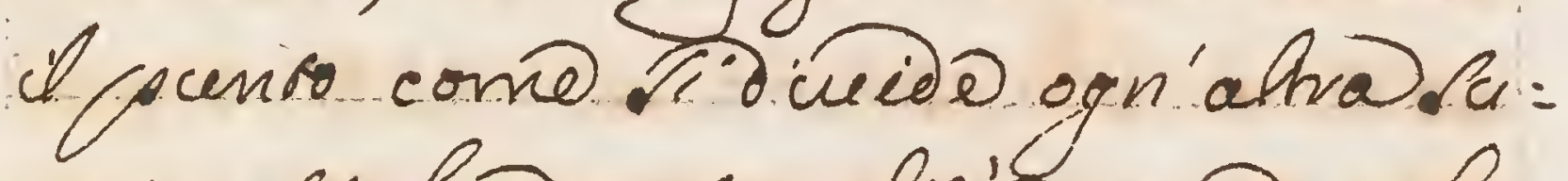
cerpavarolaño varimalizáb come ce.b.

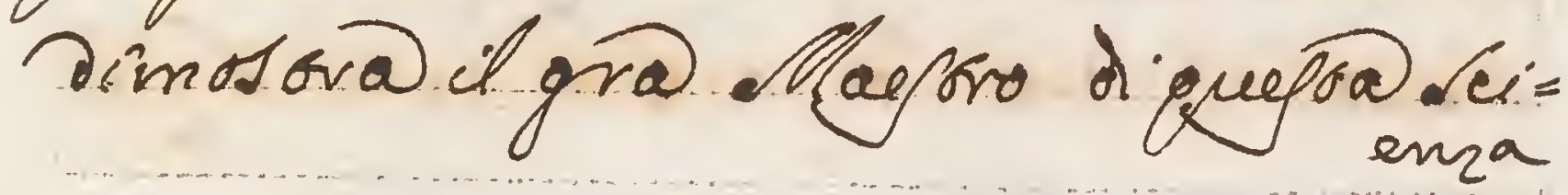


eñoluctionel firro orauo allapro. coossionesrana.

for@mi concurva' divor do: wrived ch 850merra riagrerina:

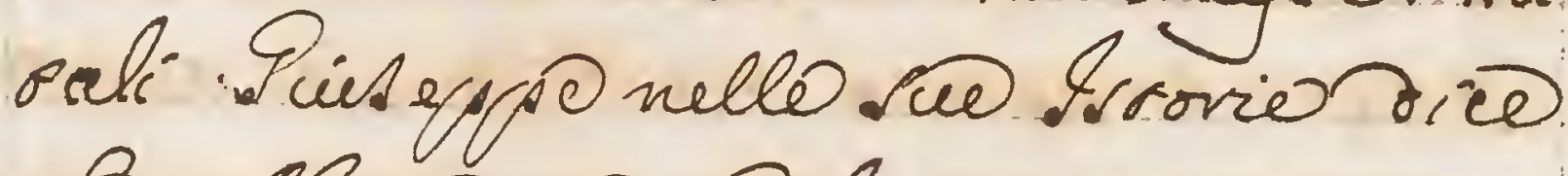
che for añ nofil il fumo visiouar :

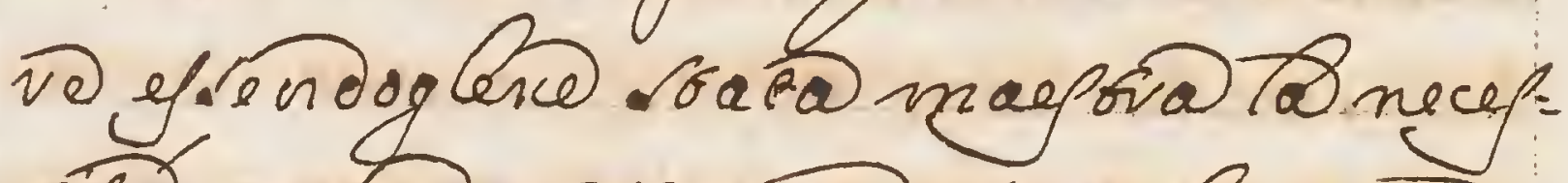
cita quando cirrondi in fan am

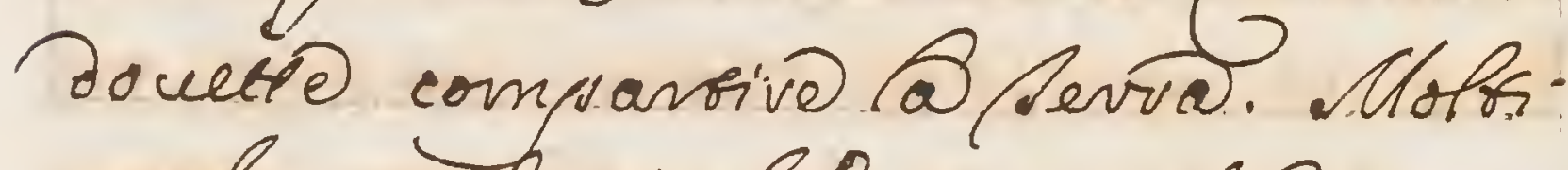
uoglime, the a' al giviziari il brogno.

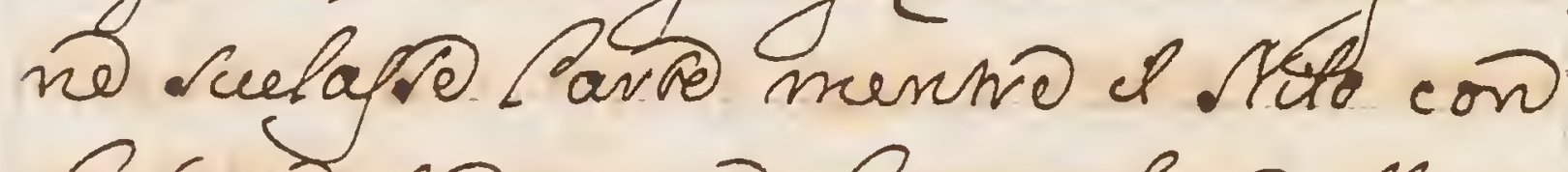
Serete ko gran foi gonfo delle pro:

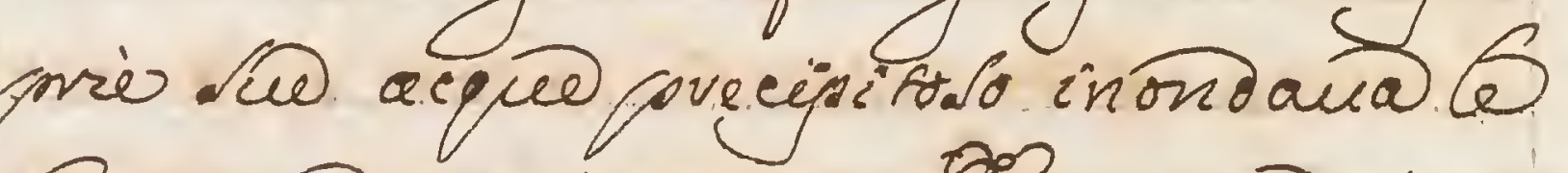

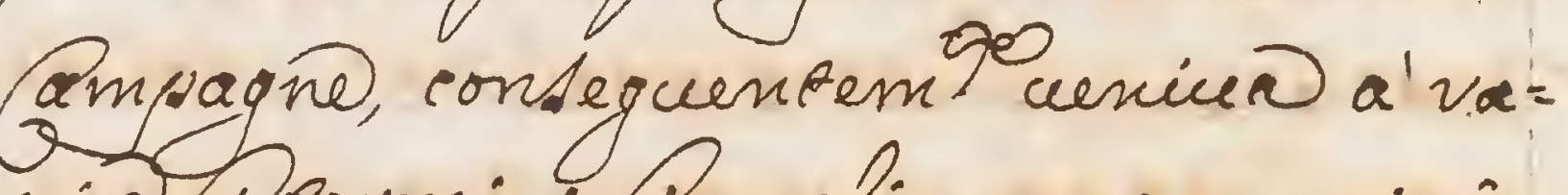
pive lipermine, liguali evano angine all'umana ingoripia, ev evano forringe Fefimony ato ogn uno confeparano. ilsuo, Dunque to forgeti quell onde 
30

sal ovorancellando Pacicifioni offo.gandops Sacto dell acque, S vimaneño.

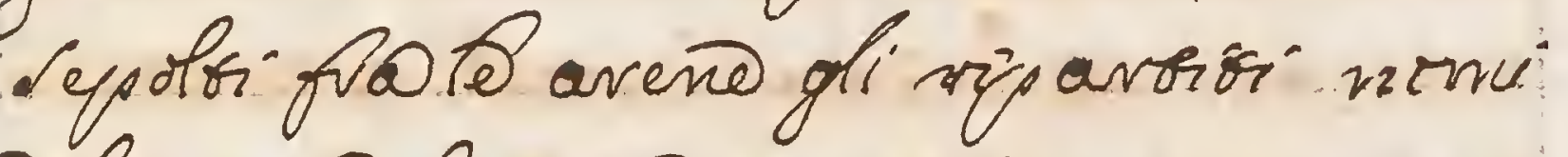
del buo, e. ol mio bi qui no vilorguano nowe pevourvatione as sirs che ne cagio. nacuno infincti Ligigy de quali per vi: accordanive Bisevepange Sivaceoman:

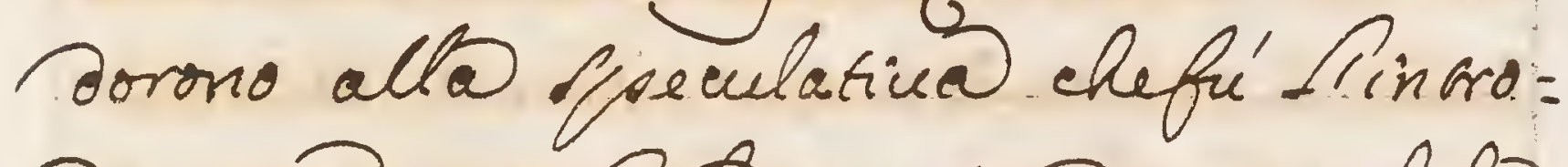
Dutricecser farto conoserv in qualcho carbolofomeria uevo itromenro,e conopontionato mizropersor acuifioni picé giufro. Diquesto Lentim: fu. fribone con

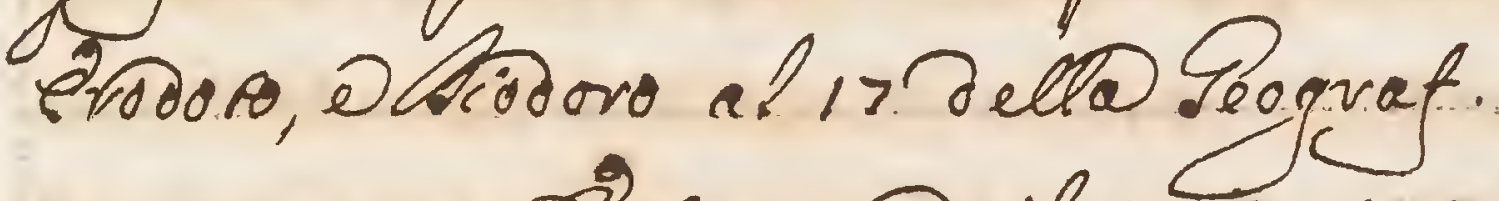

P.equndoil mio vaggio = mamidaciónororonos divcei. comprentevere non enja bugsone Anow Sormont quea. vienjobela Gomeria. Arianiamo nell' Peclesiassio 


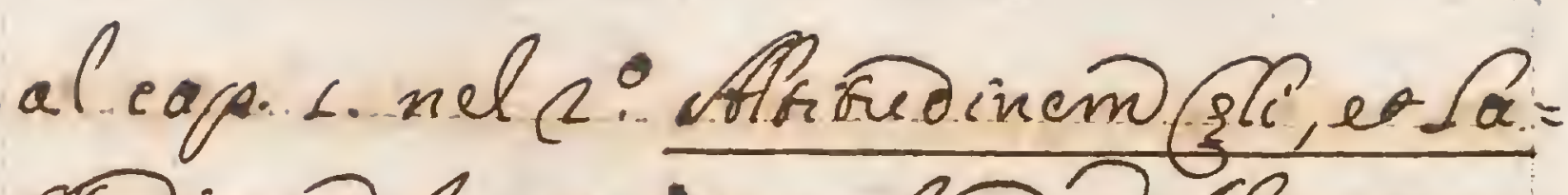

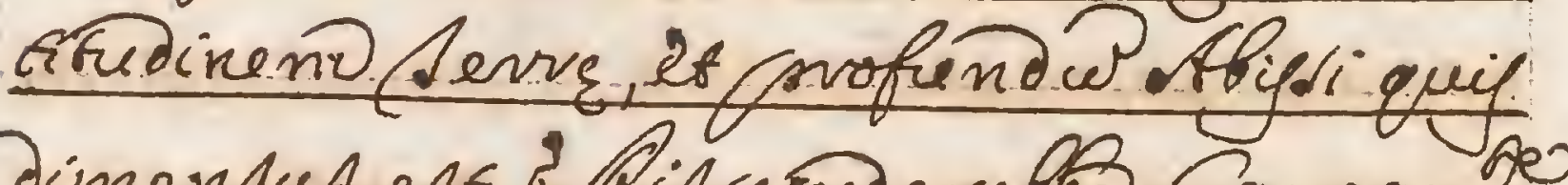
dimensus est? Pisponderebse francant?

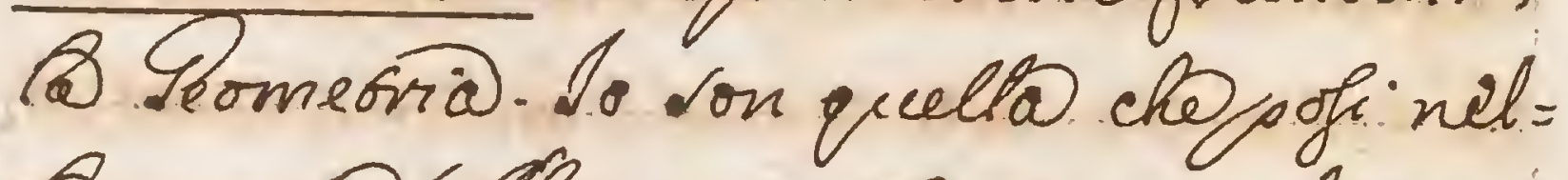
A mani J'Agranio, e hranti alwi in = gegri madevmi 'istroment bo poter Re= golavo proportion bi quanbirà, opico=

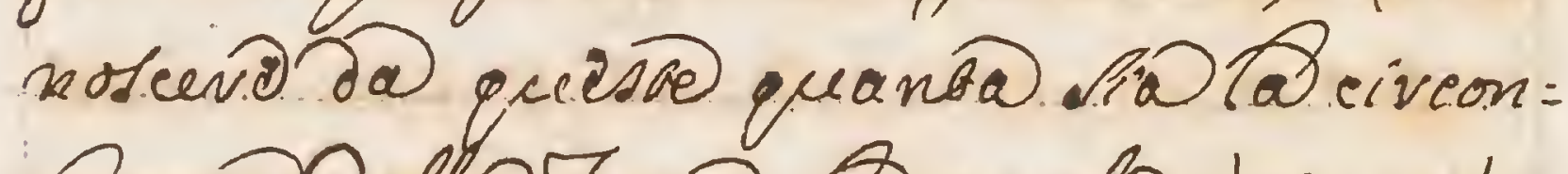
fevenzardello'Terva, D quale e' frent'un mila, e cinquevent mín Ifaliand, ece ogn'uno hiefsi r'estenoo millo päsi

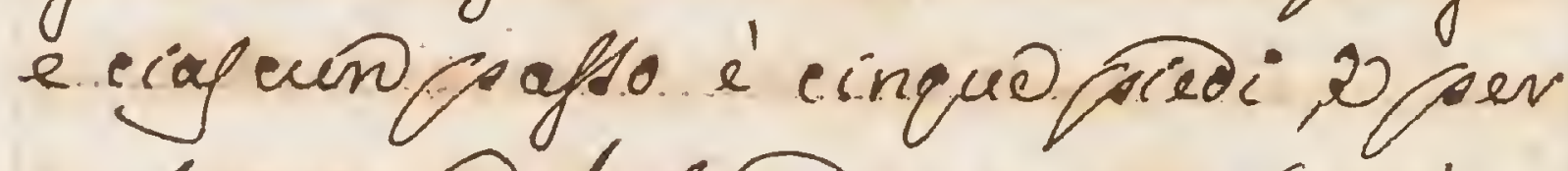
contequeniod.cio.diametro. Lava'qua =

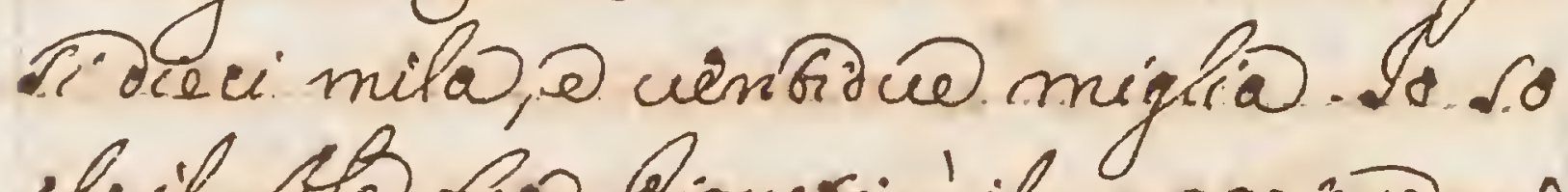
the it tho fro Sicanesi e' il maggiono es che que to gran Liminaro ona aluce al giorno ilorno al Mono. Onle mis fevoportioni l'aceanzorono Iingegni a' pe = 
39

netravio che il ote e'maggioñ della Jeño cenvo Sefrantati uotre; D. Pellefifte del: Aprin@grandezanomagiovi del: laferra cenroquindeci; Sace nouant. uno; 0 nouartacinque colb arevno.

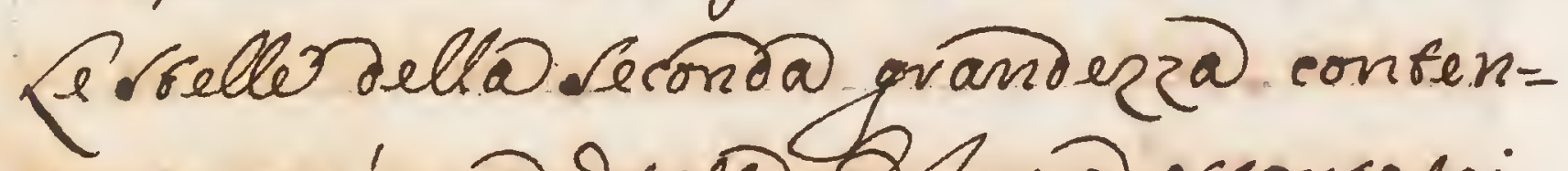

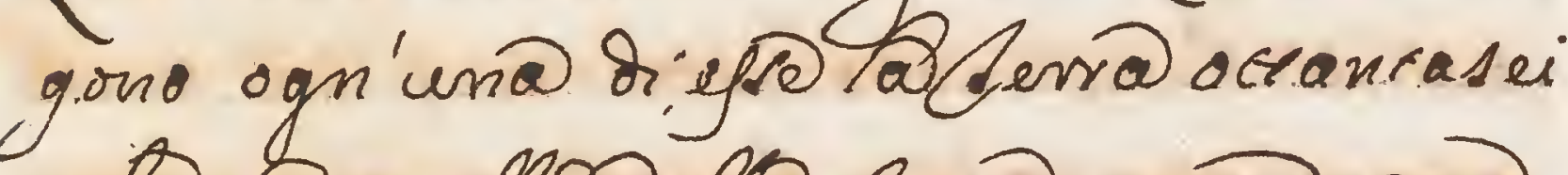

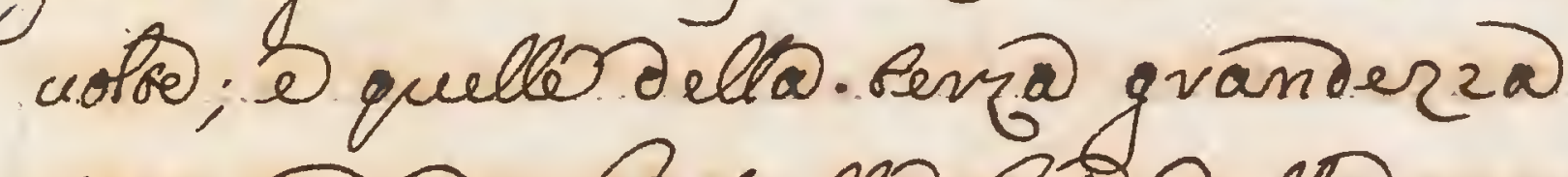

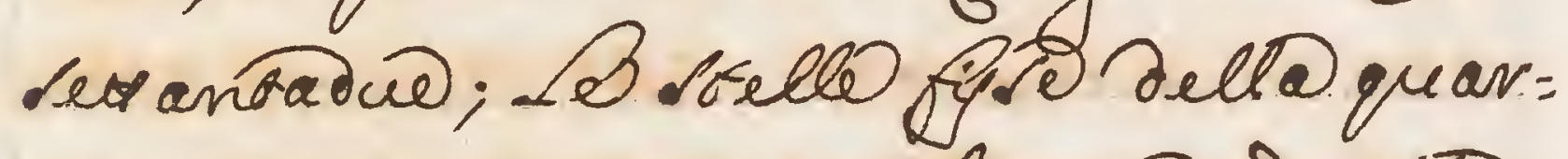

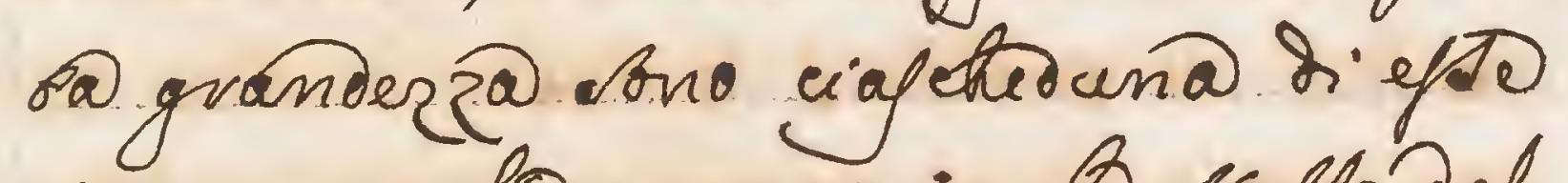

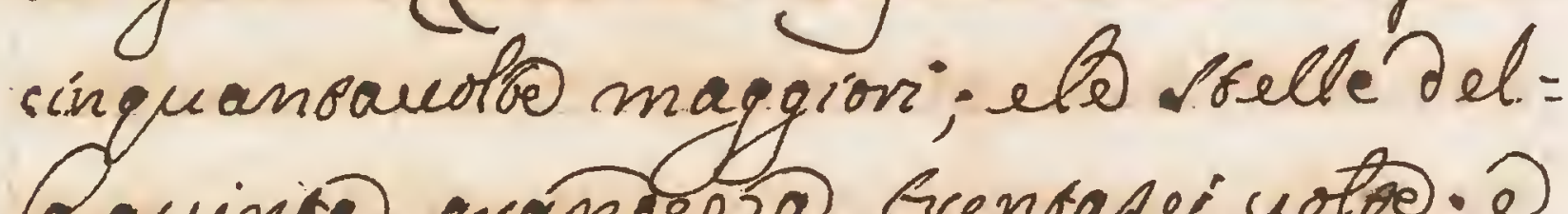
Qquiniragramdeza frentasei uoto; 0 quellordelloreragranderionini. Mar =

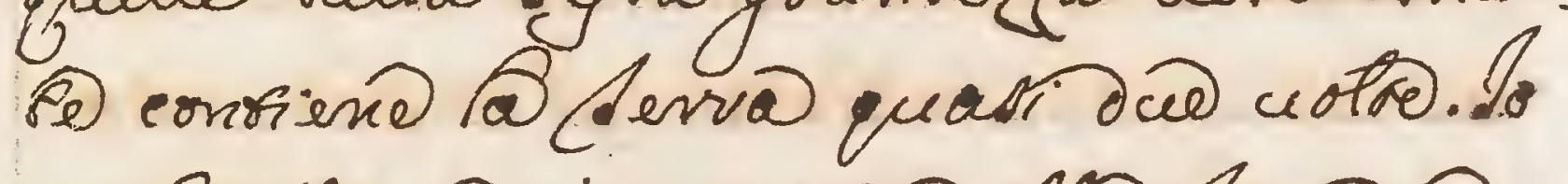

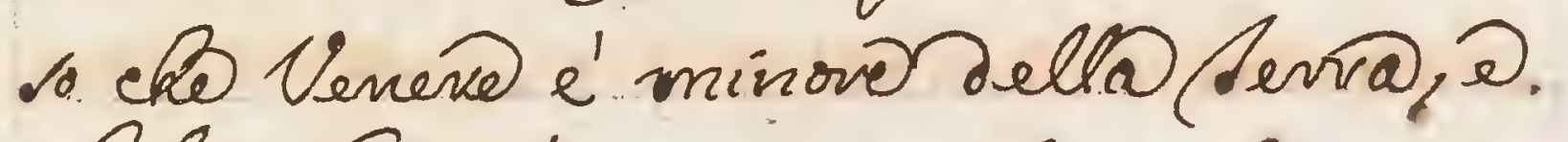
cula una équati uquab à Venễo. Mercurio fro quejo gran conquie $\delta$ : Lampadicelessié Bominove, ecle é con= oncuco 
Eenuto_dalla_tevraquadioromila cento quarantative uote. So So che fra norjeil chell@_iñ S'conta un ciaggio 8i migiva cento Jefra noa mita quatro cento unzrjette; Fino al ietsi Meveusio ac ove: sento Rerici mila cinquecento cient'obo; a' quetri Venervo ocrocento orvent'un mita otrocento unsigei; to quel del Soto dei mi Boni cinquant'otto mitar duecento ocean: banow; a'que/ hi. Marro Sei milioni cen : bo e otromita quatsocenro enowe 'f quel Di. Gialo quavantaquatro Milioni quat frocenvo Setrano da mito Sei cento, o. unticinque, ofino al Gat Sa Gevno. - etrantadod milioni cenos setrint'ote roita quarsocenro quavantaquatiro; Pper valiva all'stalia fera ceno mi= Roni setrecents deftantacingue mila 
40

cento nouansanowe; it altanona ffero. Jucento, eren milione cinquecen:so frentasetro mila quatsrocento, 2 now. Lonquella cho hapubo mifurant raltezobefali, ociveonfeveniobel: hberva, ecer consequeño diquerfa. anche 8 covofond a dell ofips.

Ce cor uoletre apprende = ve con Porecchio de Filos sfi, e mifurare

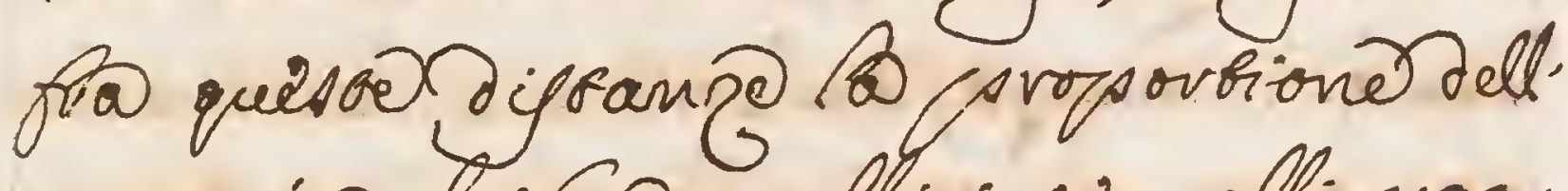
armonio che frow quelli in serualli vac:

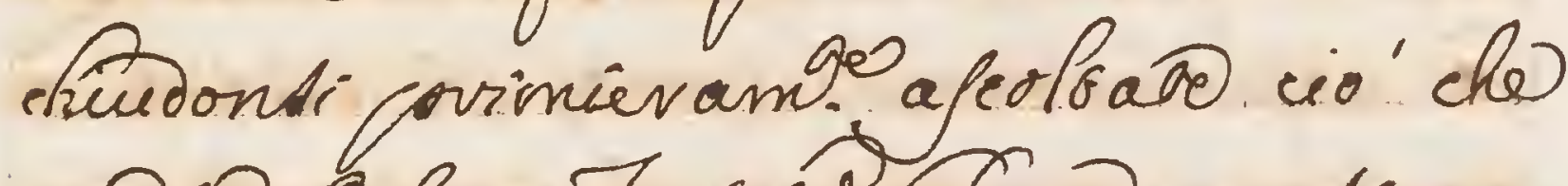
neวío Orfeo = Iurobi glum quadi ca: novam iraval femparad = Io abfiamo. in Giabbo al $38=$ quif navrabir grovo

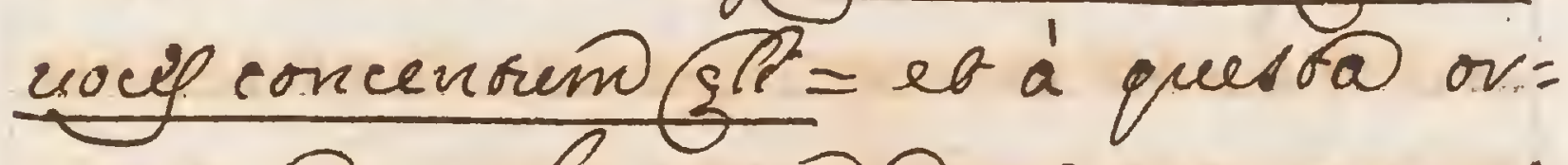
ganizåara conninasione di suono agri:

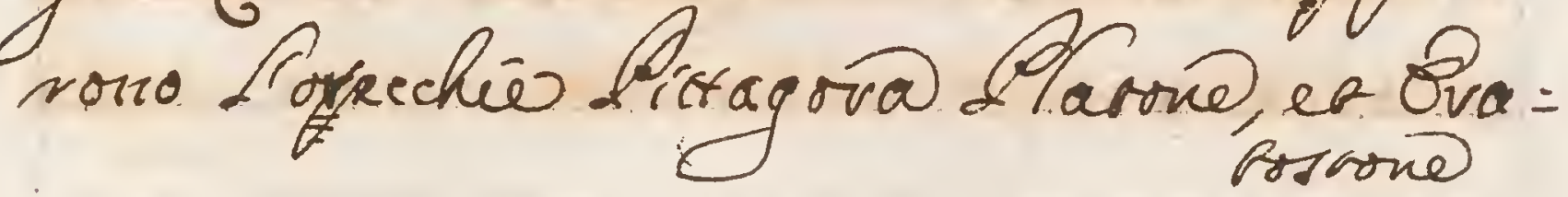


rostone, che ben comprisevo chralla. Jervallañaier ujganja di unJuono, chedell@lludica is propor = sonosiquioraua, Jalla una alla selladi. Mevcurio un semiruono, $\partial D$

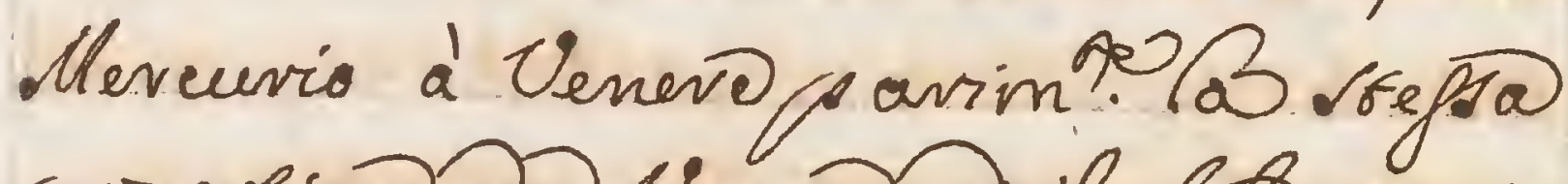
corportione, da Venerve il oto cen mi: polo, Dmizzo. chesi chiamazrap eño Dalla una il Siabafianon che e' un zuple, omezz; Jal sob $D$. Marre alme: taniro quanro dalla/Aerroalla $a$ -

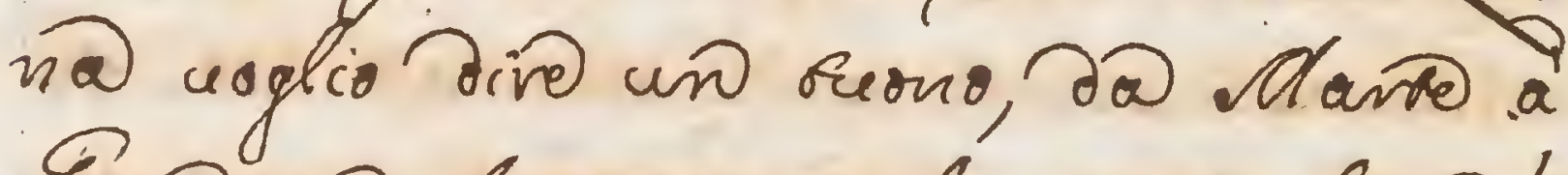
Sicue un Lemiriono, il consimiles da Sioce, Saturno, Da Sarurno al Som: mo Gelo il suquiplo, o cor'dal cielo rell

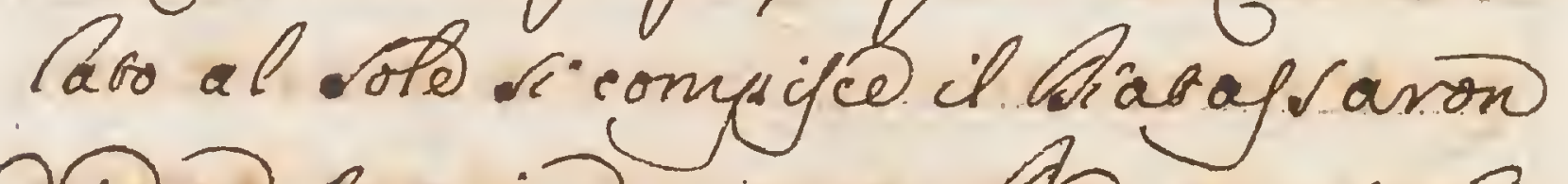

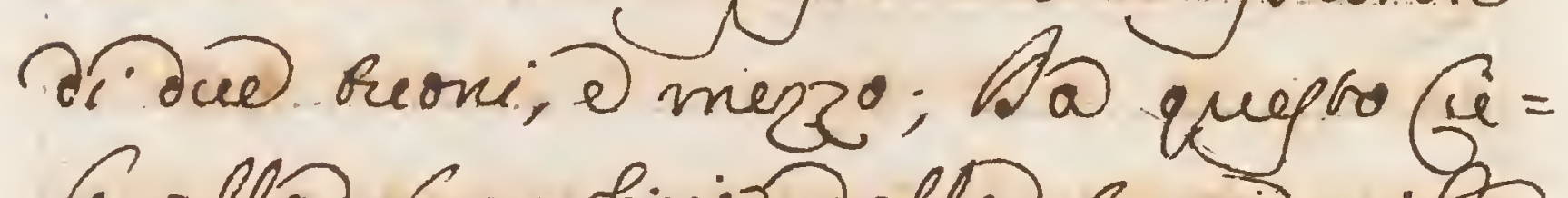
Tollo rupenficio delta ferras mi:ha 
41

Sui Ruoni, da quali no deviua@ Sinfo: nia Jel Aia pajon, wivimane il Sesbile,

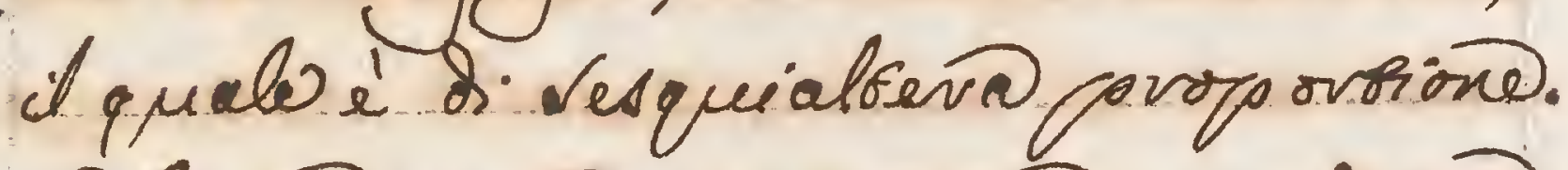
on B Wu jegte proportione vinuence B Peomemio. Atriolabio con eui infe = gno' a'mifuvare un gradrol gelo,ed

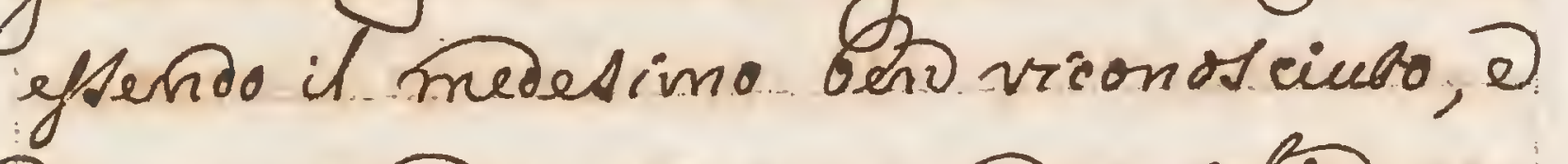
i. poi entraevaro quanto miglia cor:vis pondono a'quello, sie' Citrouaro, the ogn'uno iq quessi imporfa in ferva of =

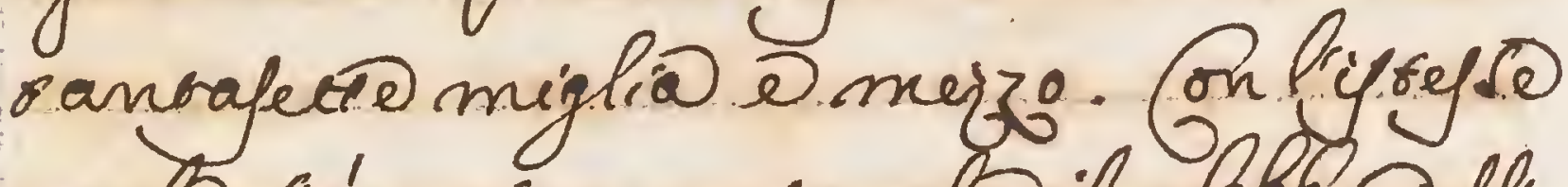
vegoto sín vinuenuro, che il grbbo dell acqua é dieciculo maggiono deltaser =

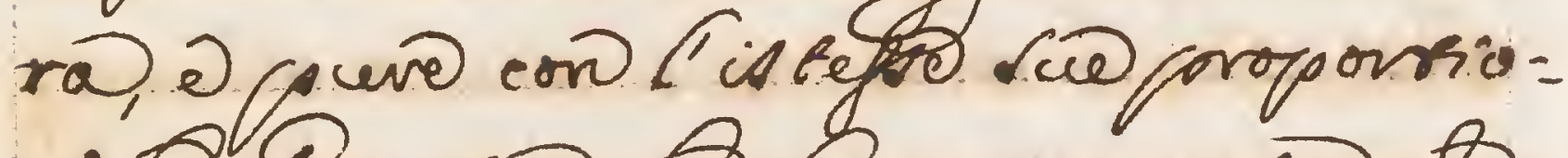
ni 10 Tomerno hatro vinuenive che

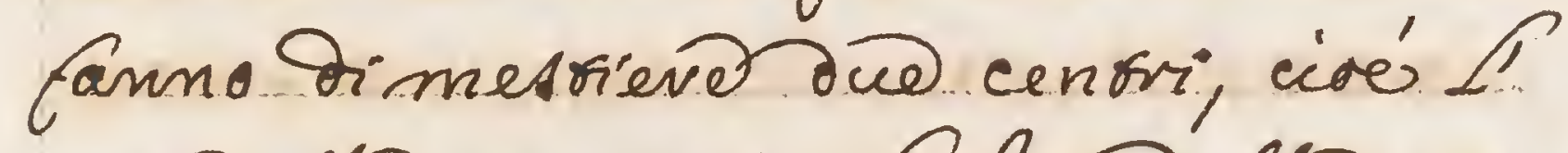
una.bellgrawizo, Ralno dellogvan:

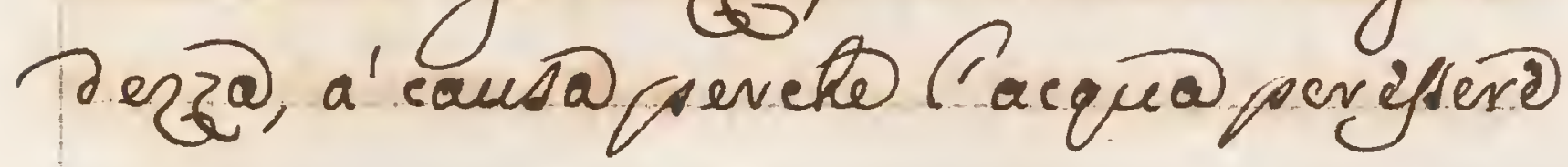


cuis grawd dell@_evrodiderodiscen=

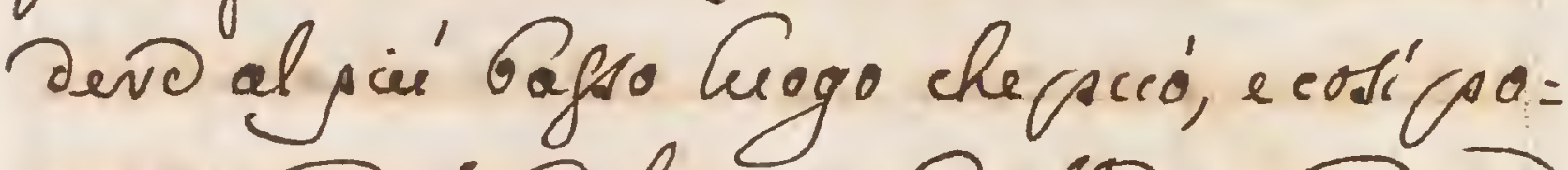
souvandosi del cenvir dello grandezza

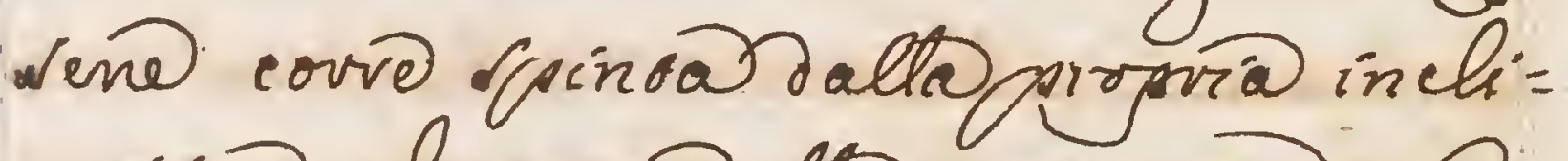
natione al centro della graweza. on it suo appoggio s'e'giunto a' capivo che Ptria édue coobo pin'dell@@erva,o chlorfermad fuce e' parim be santo cic' grande dell tria quanro dicem. = mo tr toria efser magione dell' acqua. Fra que elements anche fro Loro w'sicomprende unapenfetra armo= nia), aquate wi sirieonmparalle fovo Sasi, e dalli Foro ang ofi, sanix che of = sendoci frail foes, ol Pavia unaporo. forbione austa nello bafi, el a Sesqui = alsera ne'gl'ang oli sotio: 2 ol ofro cio ne'

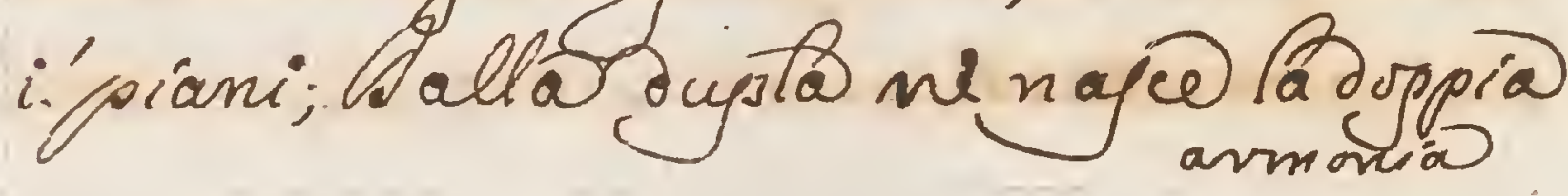


42

avmonia del Aiapofon, e delliapento,

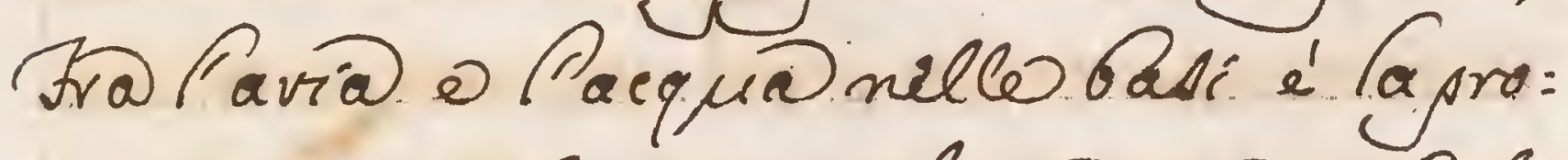

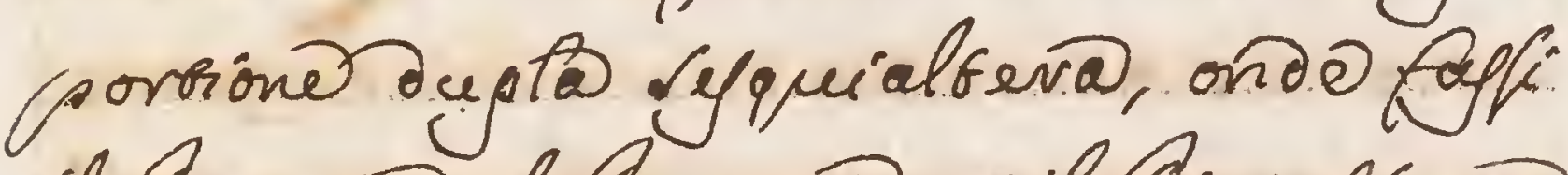
il Aiapaton il Biapente, eril Aiacapowon; (a) Jupta ne th angole, walche ece un alvauoliaisopason ra Pacqua, D

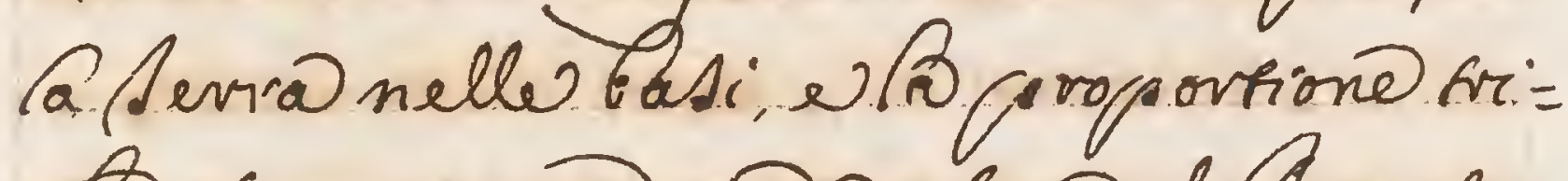
A Sesquiteriá onde Arge il Aiagajon illiapente it Airepof avon ni gl' angoli

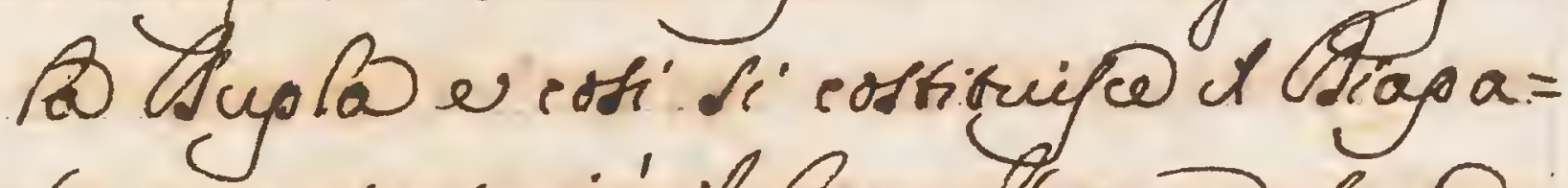
Ion; con ruto cio' il freo, el 'aequa Son bue elementi fro foro confiravi; e nemici, al =

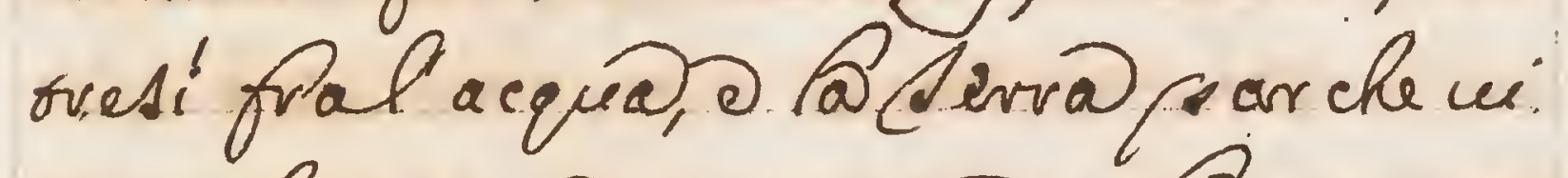
manchi in un cerbo modo 20 conto = nanjoviroundsi fro foro regugnan: io Liqualitás ansipariche

onlaguidadella Somenta cièmon arofin surv/o ffere elementasi 
doweprincigia @reqion culeste,conta

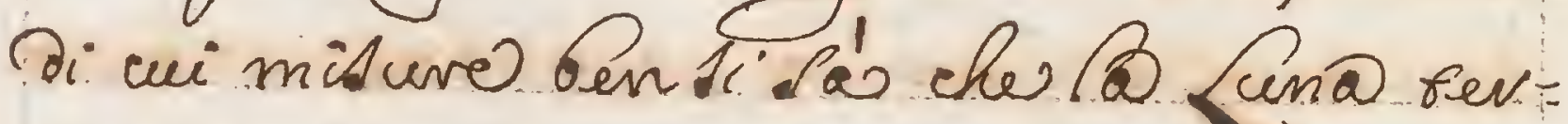
mina d cu camino in cunvifeore giorni; equadi dréci ore, o cho Meveurio, o Ve = neverdi weluciós nel foro moro ano poco inferion al uolo Del ote, eil Resdel le = mo il Monavea de pianeti in orecento def = ranbacinque qióni \&quati Lei ovo ber?. mina il suo givo; Libs: Mave in due anni compúfell suo coss, 2 byvan ferro:

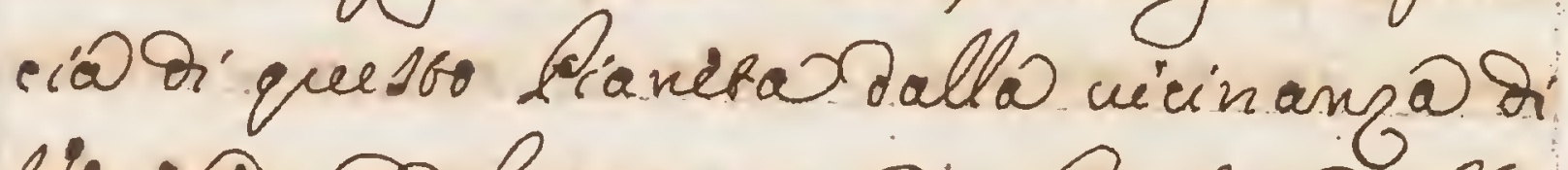
Veneve vadolcito vimañ; Sesto.fe: va disiou in boreci anni fermina il Suo camino, D. Corevno in ami breno@; m@efsitoqueforanocosellariona

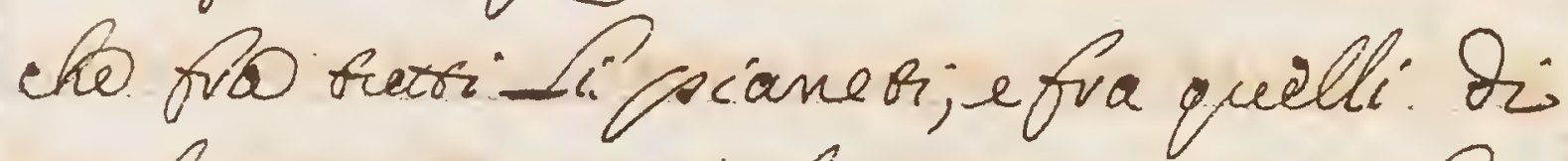
maligno afpecto e'illeefimo, coti $\hat{a}_{a}=$ pienzaciuinal evedermiofra. Nharbo e 
43

Naturno concestellabioni infelici ci colloco' Goud pervon farceno prourvo tan: ro fricaco it anmo, le accio che conjuri benigni influfsi contempevapse cosi afte: voira dell'uno, chela malignita' dell'. alwo.on@befoSeomefria.sicompren

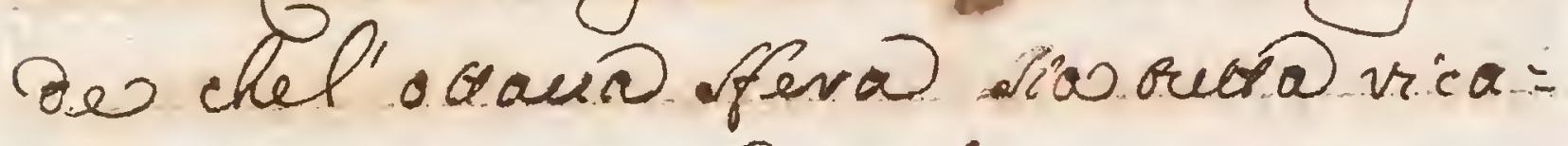
mat@Diselte, Aquale cuien Denomi:

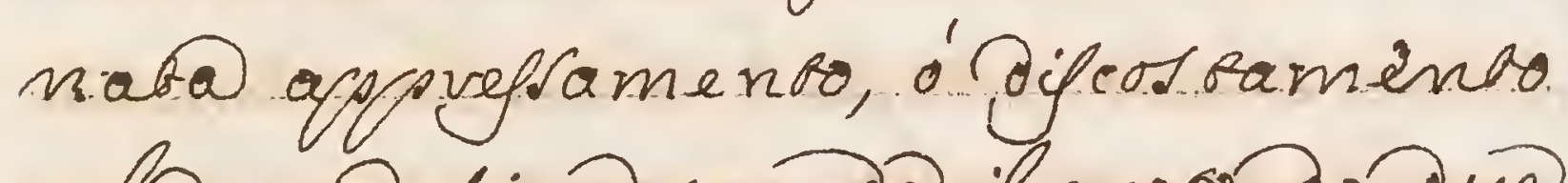
elamedetimoprendo il moso da Due alme fero. cupinzori, món now rzimañ

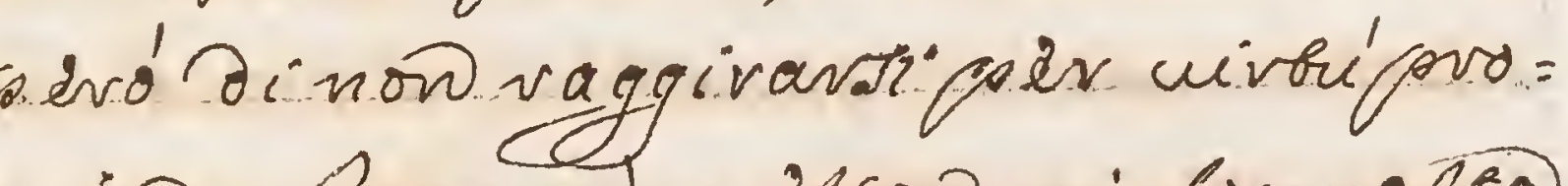

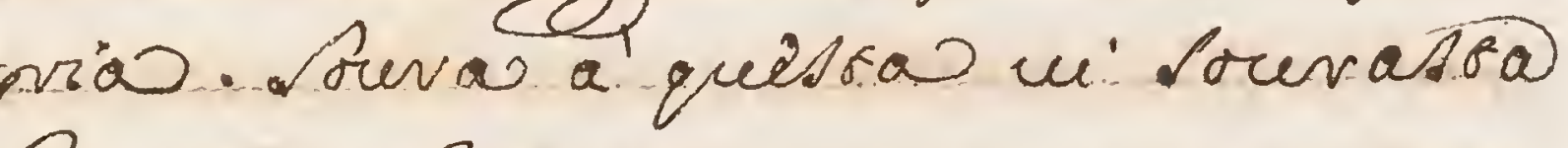
3nona.feria, oue non u' di consano

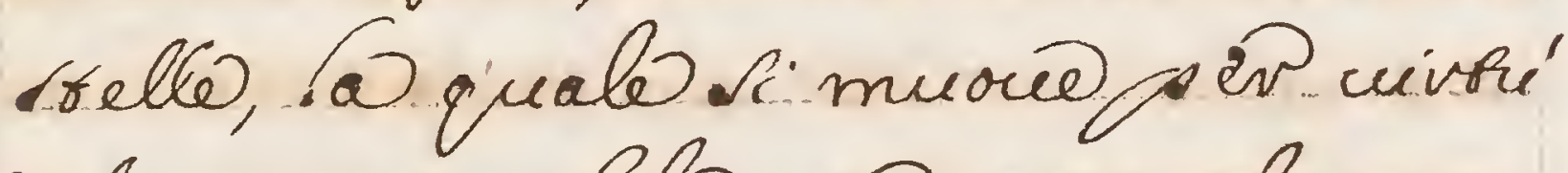
del fovimomofile, ma rutro al con = sravio del medesimo cioó dall' occafo allioviento, e col duo vatro orad seco 
bute Palme frevo infiriori, o camina ron Eanta grauita, opafto si Lento cho in cent anni à peñ compice uno olgrado. If primo mofile becto cicido

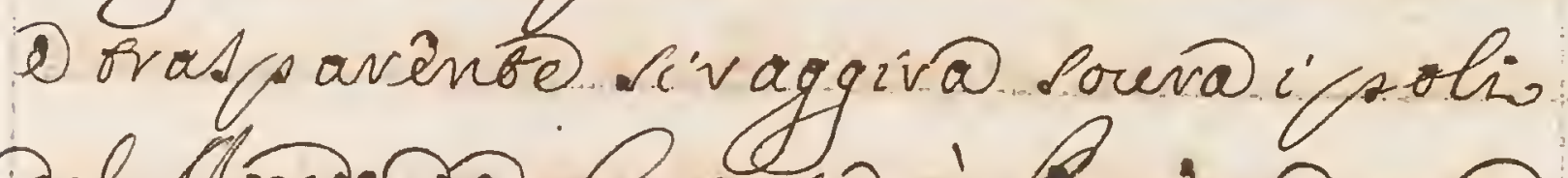
bellondo@Luana à Ponénte con pantalocibá, cho in uentiquatr'

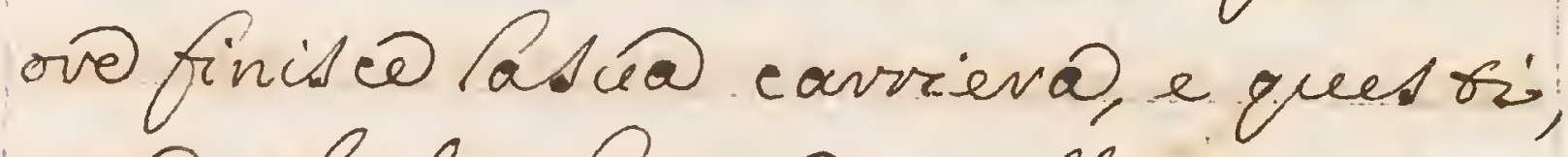
come gl'altir houn intelligenja ofjif = tento che nien chiamara anima del Mondo. Ma io snza essermeñ auce: Dubo mi visiruo güntorow non Lups: conenas'andare, o cohenoo Deseviuer.

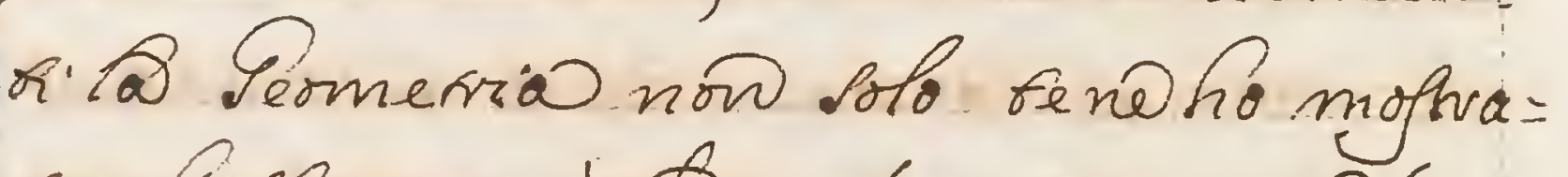
to g' 'iefers; ma' at dificú mi vimouo J'auiv= benofacto sosomeno cke un'intera Letriono. Di ferao

Shi fucos 8 materio in que 
44

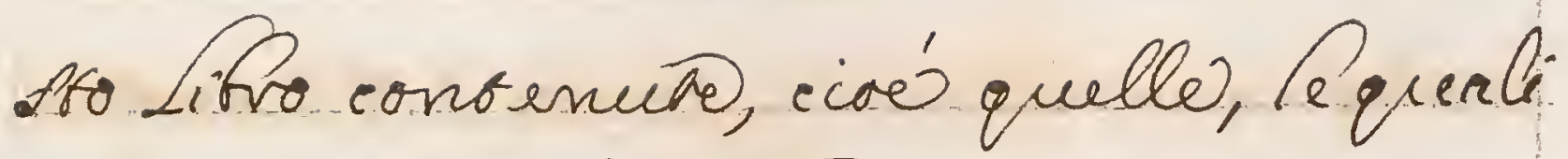

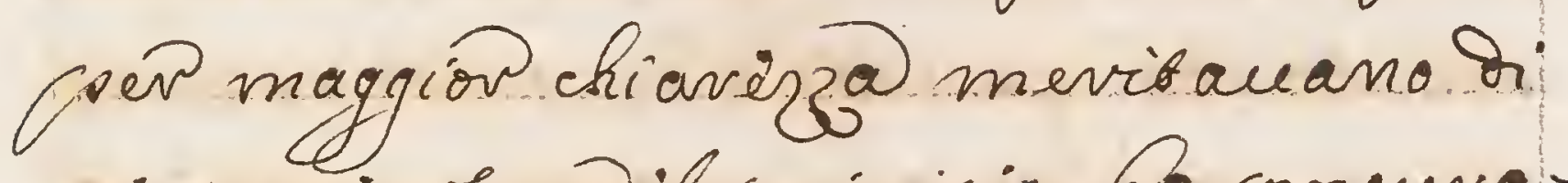
rintraceiantend il principico, ho nocuva: bo ispovosto, rava anche ben giusto

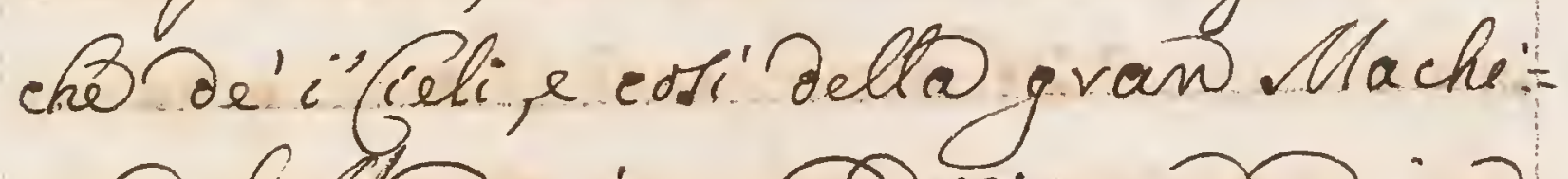
na del Utondo co ne adisi come, ed in. qual formano rupipe a'questi il na bate. a. Jerlogia insegnatari dall' in.

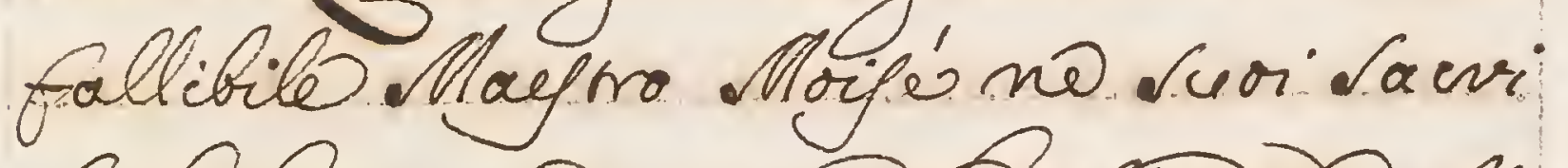

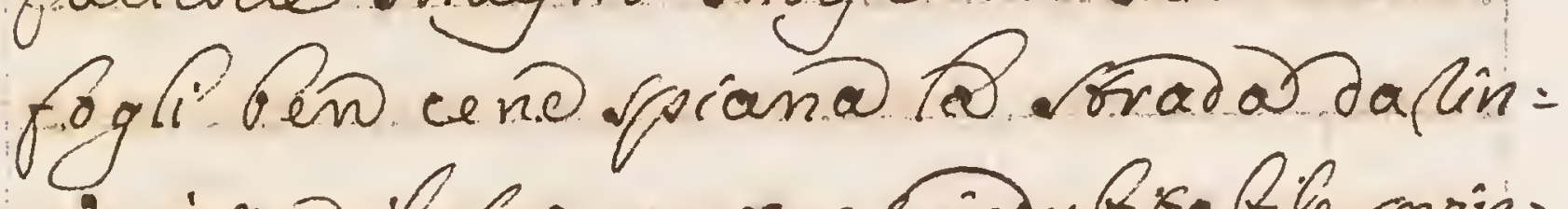
ueninno il seo curv, ef induhisabile prin: cipio,m@pen and ancora qualche

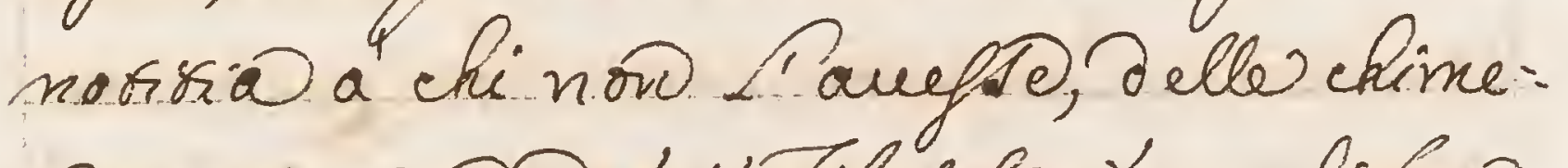
De inuinfaño da' 'Filosofi; 'qualiforo Agentilefim aña it und Atrolico. imaneuano all' sreuvo, efogn' uno.

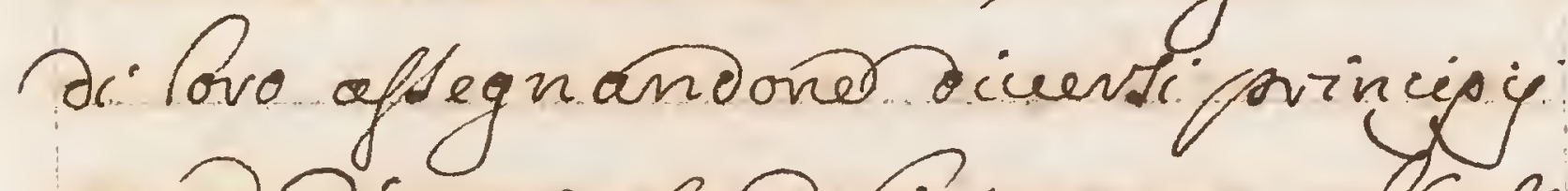
como divemo lenofig uvorno arrefi 
diutrsi entimenti como Ojfevente=

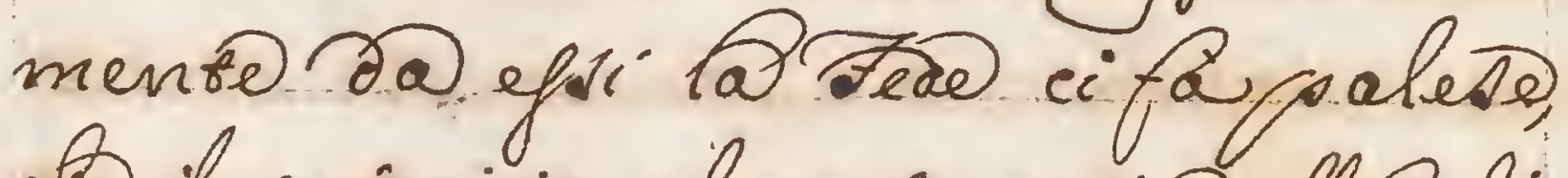

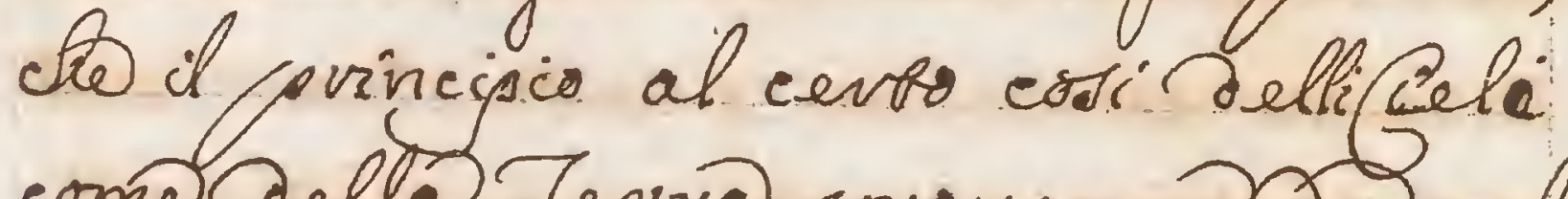

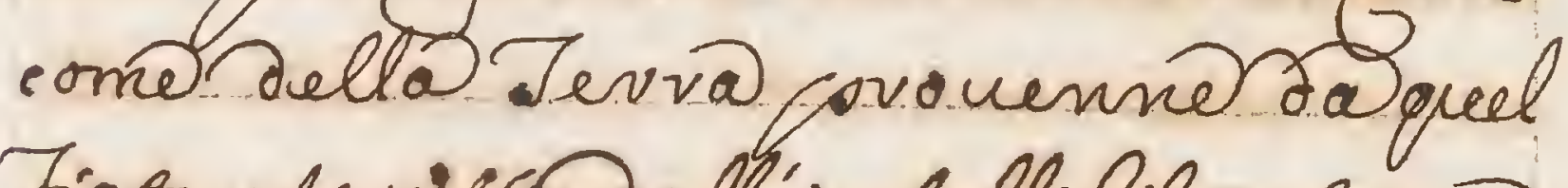
Fias esprvetro all impaliciles apervo Aicuivo ipdo Dcicit, et faco Icint Oramives@a á contavui Pfauote

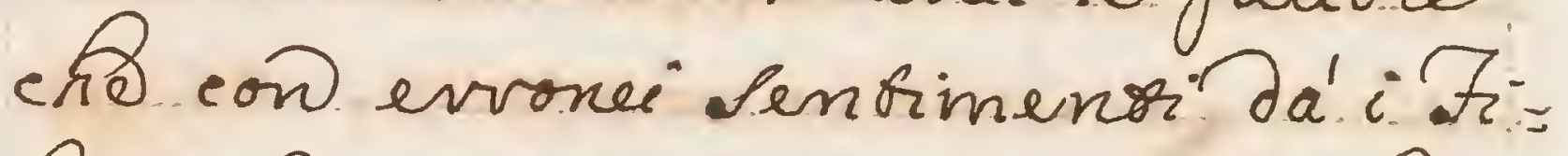
osofifervono or amandato allo Ree come pansi abovri, íquali furvono pavioirti a alli medenimi, e iv diver = Simodi confraformi . 2 principian =

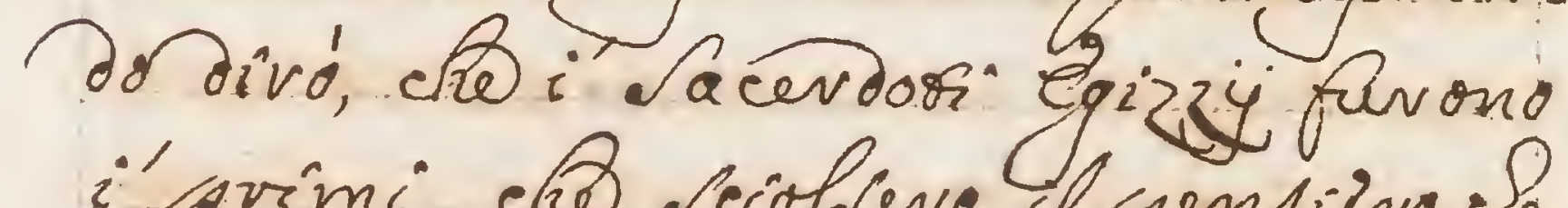
i'primi, cho ciolsevo pensitvo, ch senjafueno andó uagando a'con =

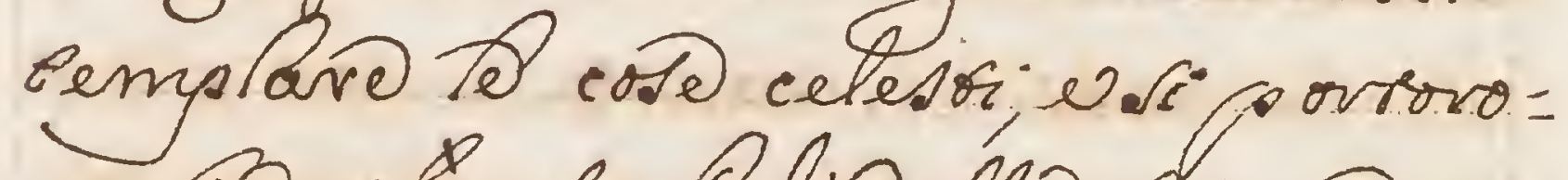
no tho cola su Palidelle. cienfen nat temarich, affivmando, cho il Gielo, 
45

wil Thondo efsendo frato evecro cser ean =

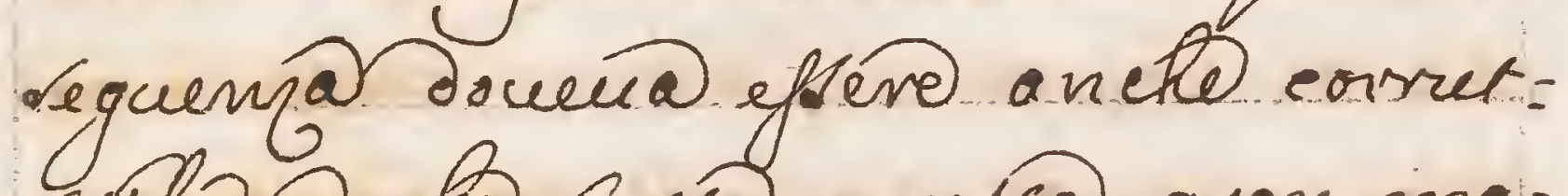
rible, eho fura queta gran ma chinanow viconobb sêp madve ch unacertalqualizo' di fevva, er

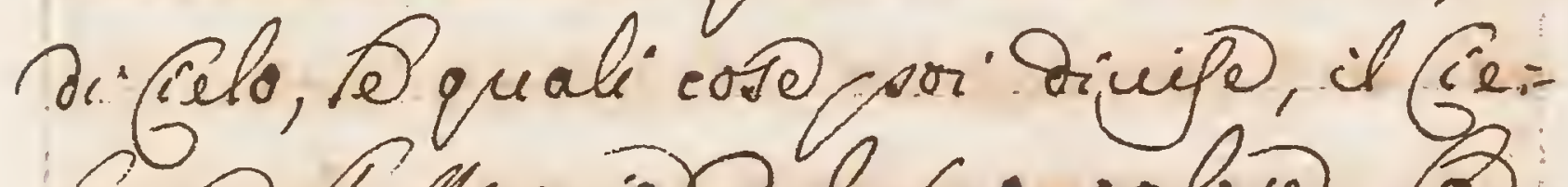

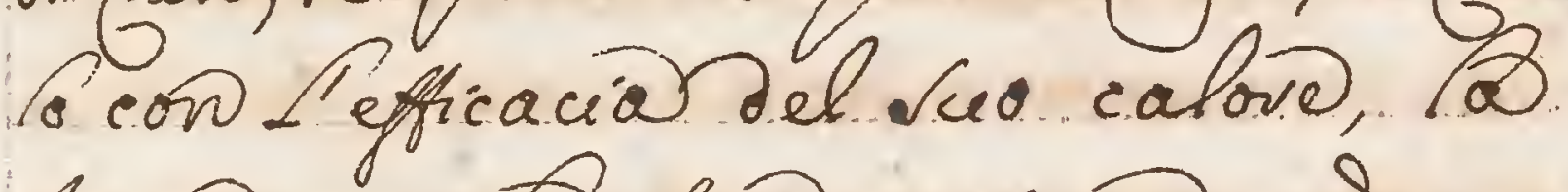
Levi@jer \$u@ nabrera, e hiquan= so coi fu ginevaro aicunnevo Sené:

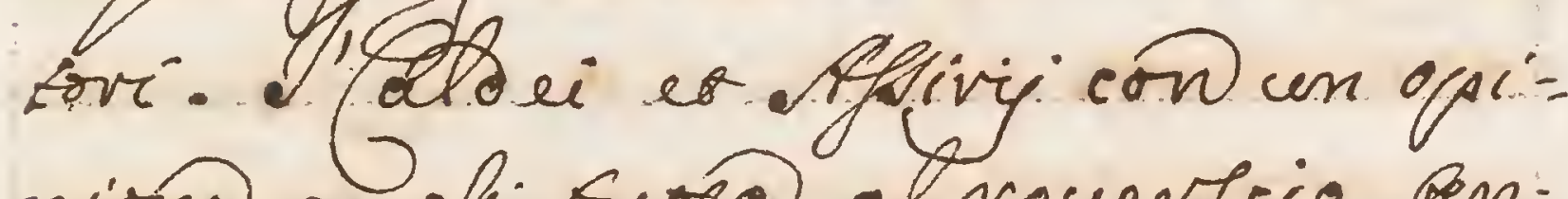
nionequafiruta al vouentio ben:nero, chl illondo. Dounea eperv. ver facalizás ezerno, a' preefor accon= entivono Denofano avmenide con

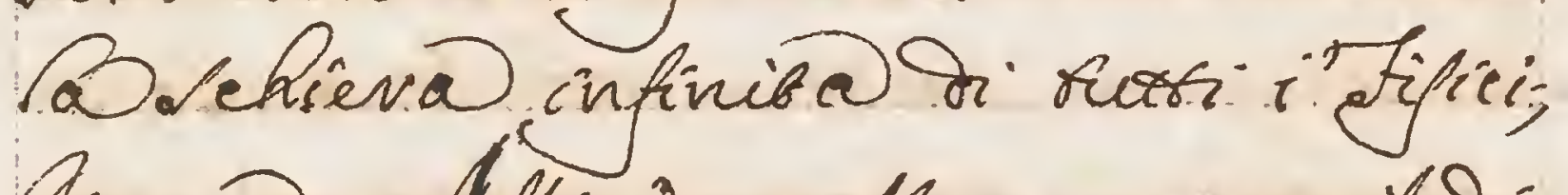
Aiogene, exdefiméro afoegnarono ith. Ai principic all'arra, et all' infinc: ro. Jabete. Milesico appogiandosi olo 


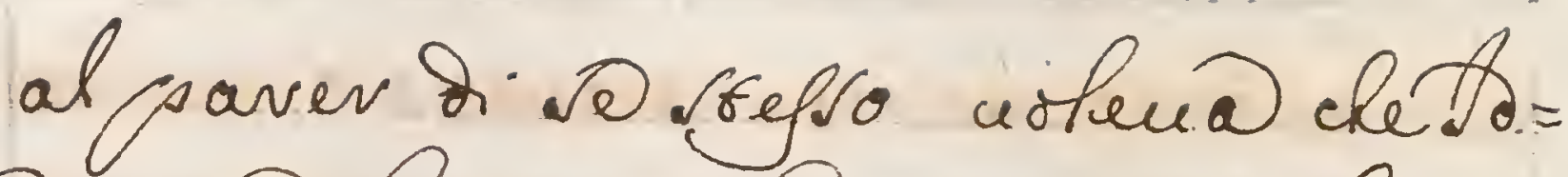
Dis con racqua hauejer bato Ao for: ma al ielo do al Monto Atreo I'ac: costí a' Talere, ma nón poré vima =

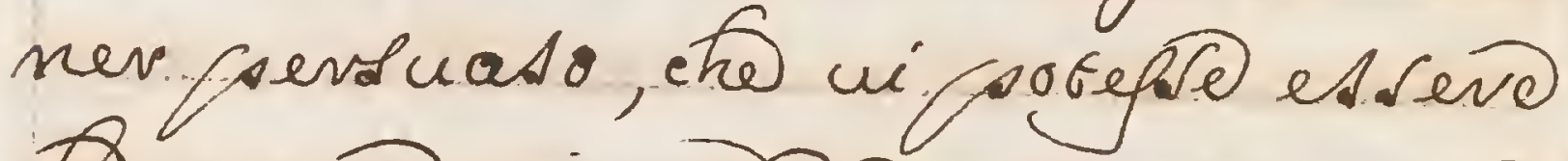
Aopvina causd, da mi quests mondo

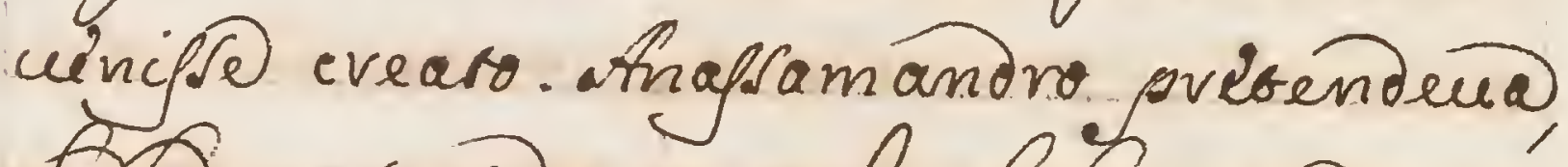

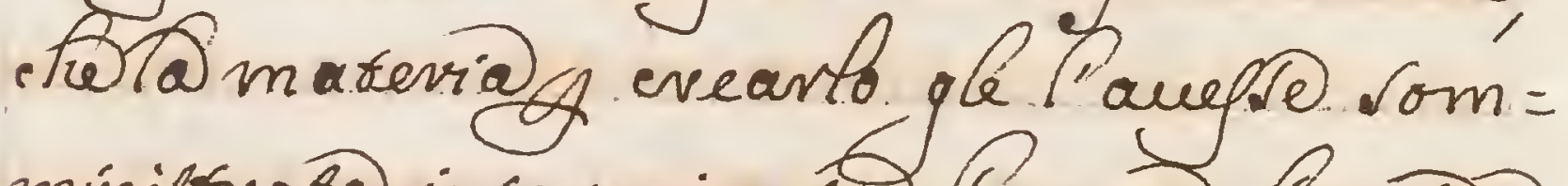

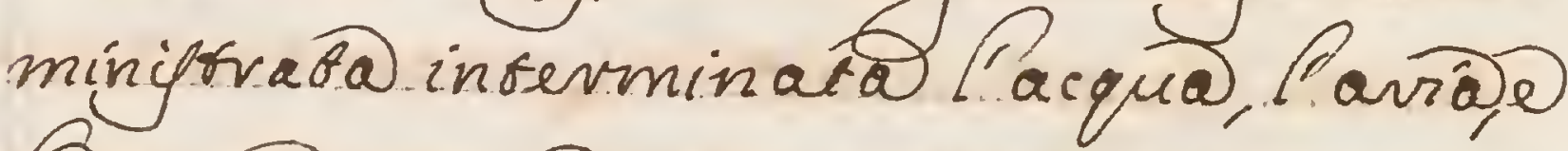
Drevio, opurt un curro mezzo infinis, etubriacabosic con tof fantrabia del fuo capviecico Delivo' con rogni u ni, dicendo chingliniti Mon: Divivisroualsevo.Avichifo favenatino.affermo' eferv un. Monosolo, ma ch torio u' hauel= Dinfusanima, comenell Vomo. Aa gl' allieui deltaplaronica ruota Siventi biv

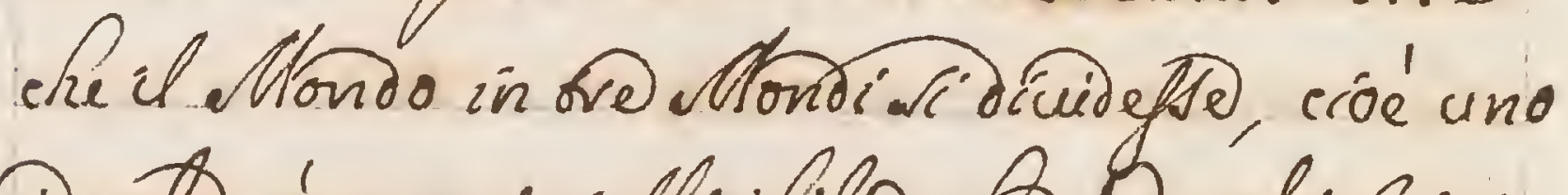
cireate, ócuevo in telligifile, cho è gliafrivas- 
46

lopracelesti; uno celesbo ouevo Gellante, ch' $e$ if coryso proprio del ielo, uno elementave, - uevo acuiunaro che questo da noi abirara. Znione Gzico uolle, che ipvincisy Sogetsini no

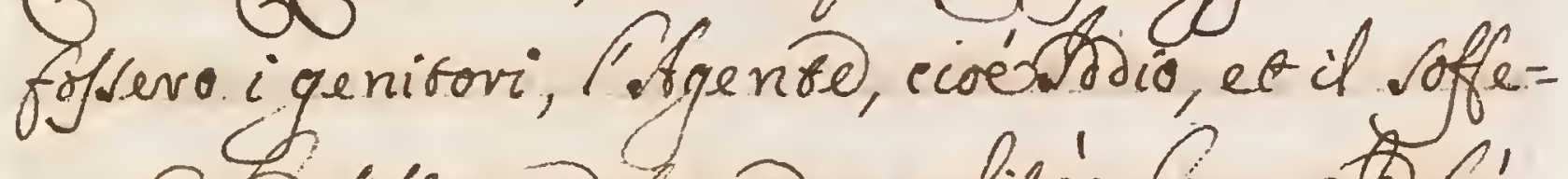

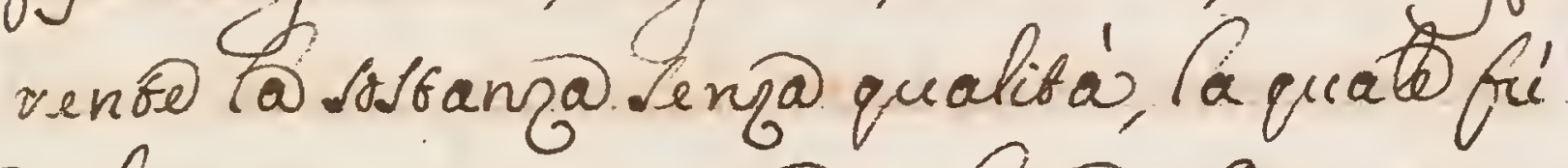
Dalui conuenso in acgiaforavia. Omevo inbe=

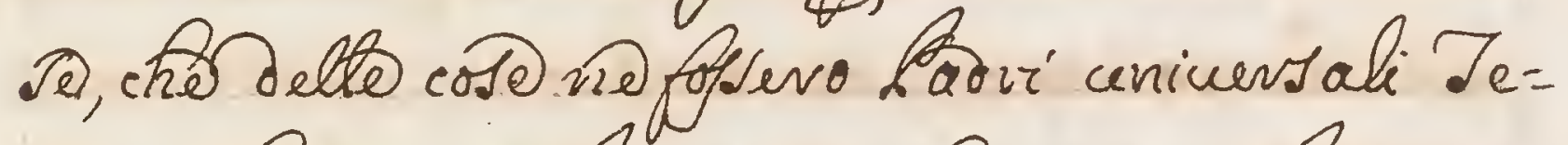

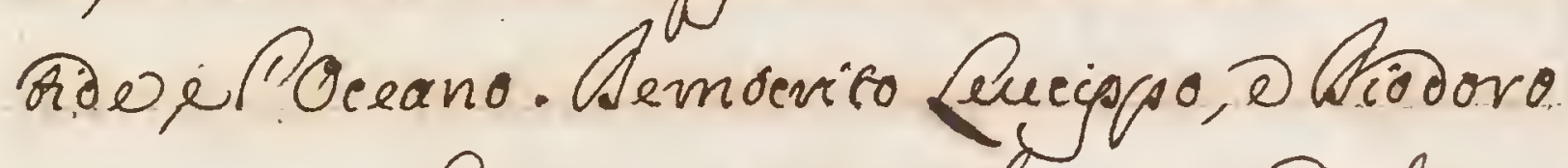

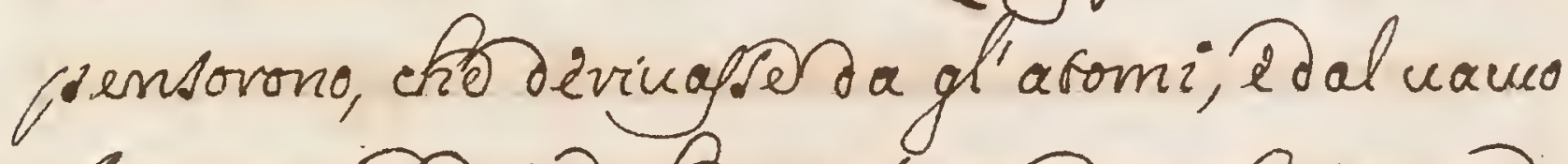

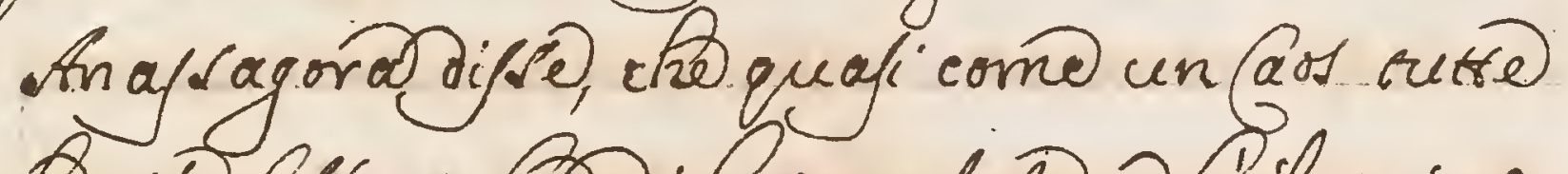
to cose fopervo fro di oro confuse, ofuid primo, chollamarena ofseynapts Panimo, Pamen= Fo. Dेंodo uoleua, cho quanro Pocchio compren:

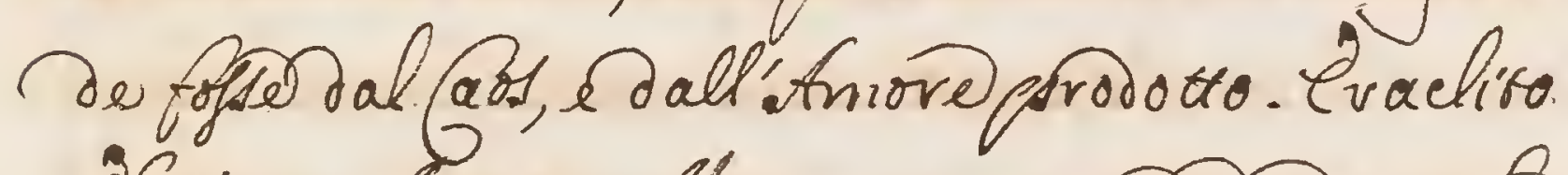
Efelis, et fipareo Matapontino no diedero $A$ cagione all elemento del foeo dittagova ativioui itfutto alte proportioni arm oniche, ed ai nume= 
viformali, e naturali. imperoch tenecia, che

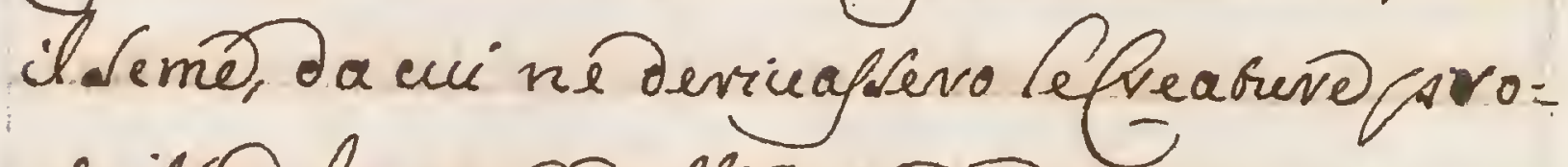

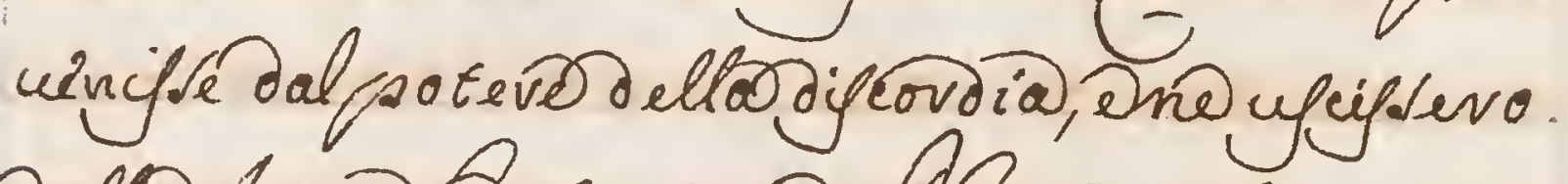

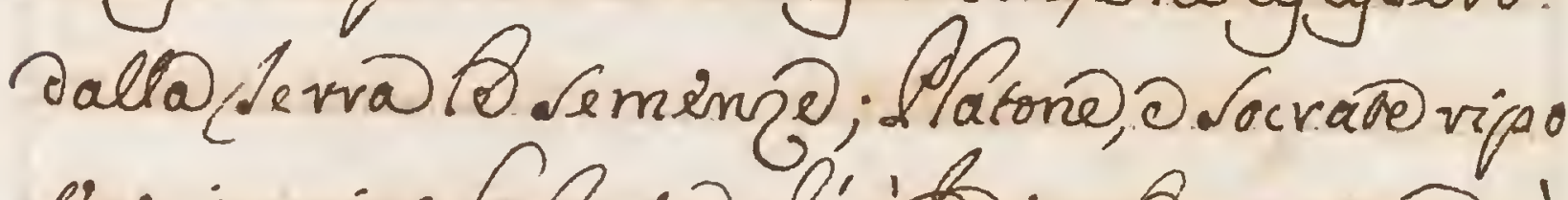

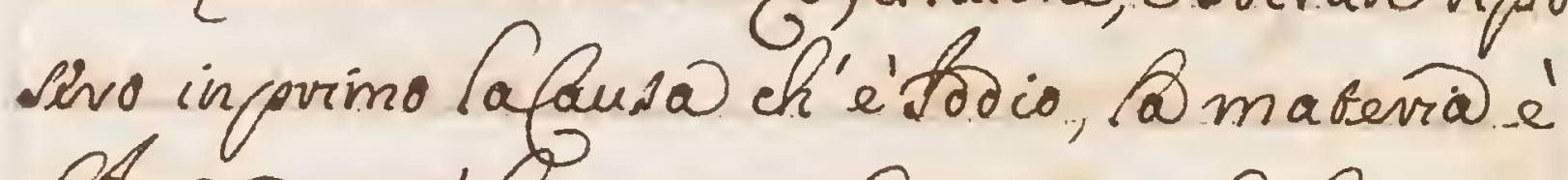

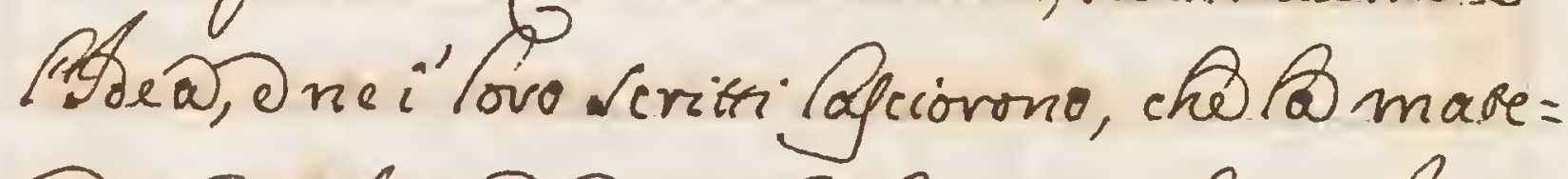

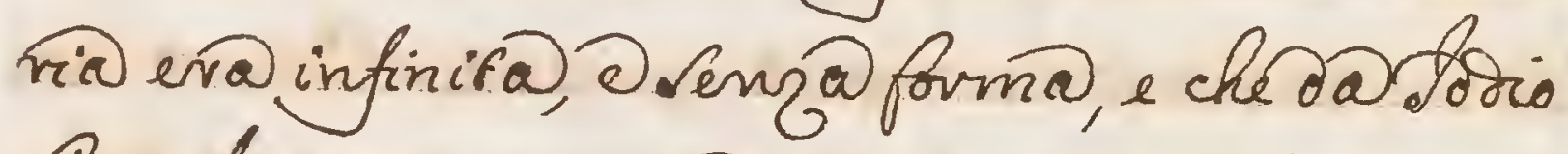
pis in luggo vagunata, Djysinta, eche cosalo Iseng fupor in elementi musara, cho quindi

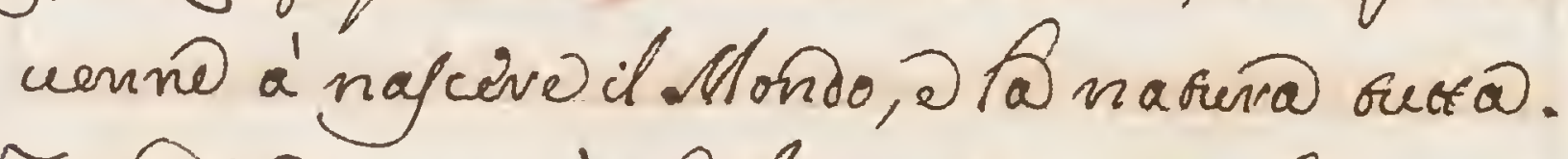
Fevecide defermino cho godi, il Tempo, Torra

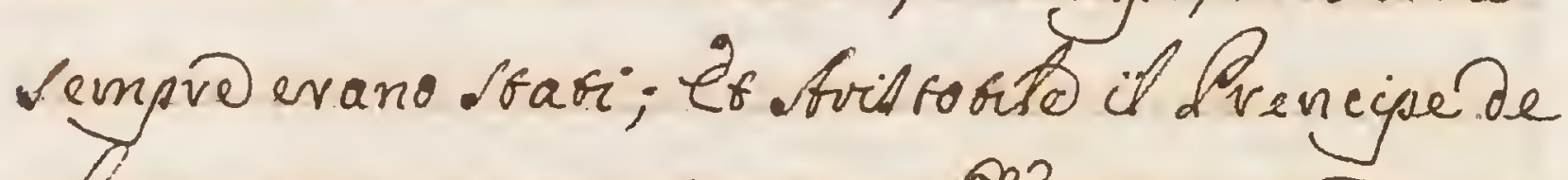
g' avgomentantifintinacemí contras bando con Parmi delto quepioni, popsedendo Affon a de gl

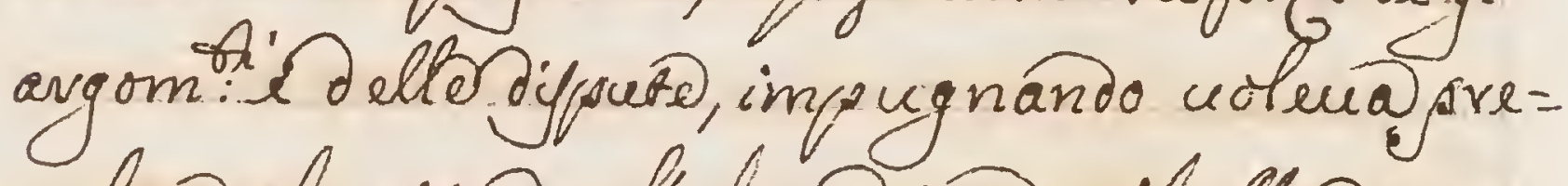
ualerve al vesto de gl alini, Dicendo, il Mondo non

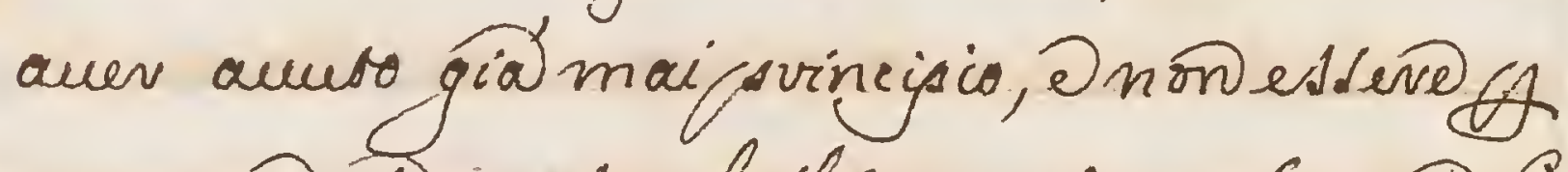
mancavo gia inai, e che il furso crearo Semprofue, 
47

esempro savá. Ma d'infiniti alfir orti parevi coofrei coov parcuene infiniti alfri falsi arincipy,

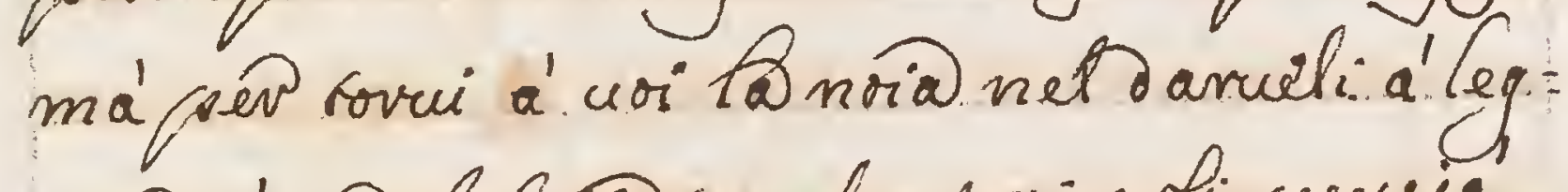
gere, à mél fatbió nel vedersuerti, sevcio no.fuggota oviga

Ovom'infrodurró, comen' hebbi. già 'lfsentieve di favei comparive locto foe = chico qualchorimostratione gromesiria

Nel Grono forr. de subfil.ow ovat: Eob Delementif do eovum motible, et actionibuf a' car ipi egliespone = quatuor wunt cauts celevifa:-

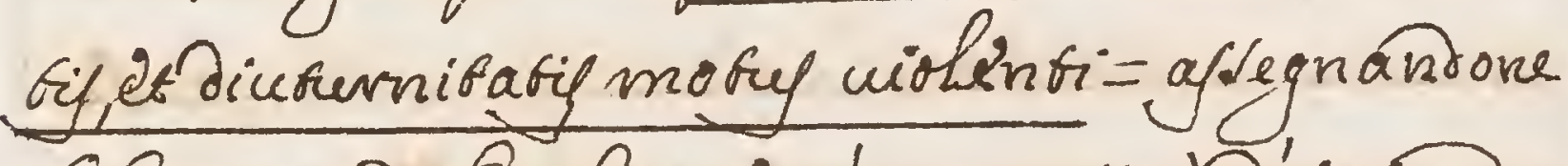
Ciforo cauje, che chi auivás cuvitsita' intender = Lecorva' viconoscerte nello sefso Autore, ove. vinceerró ingegnofisime qperationi ingenere de $i$.' mati, eper efovimene cio' chomi ono afrunto oi

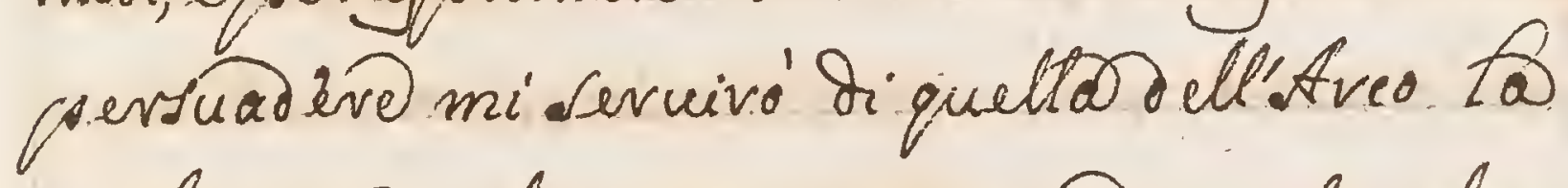
quato secondo il nostro intento Eade molto al pro = ciofino 


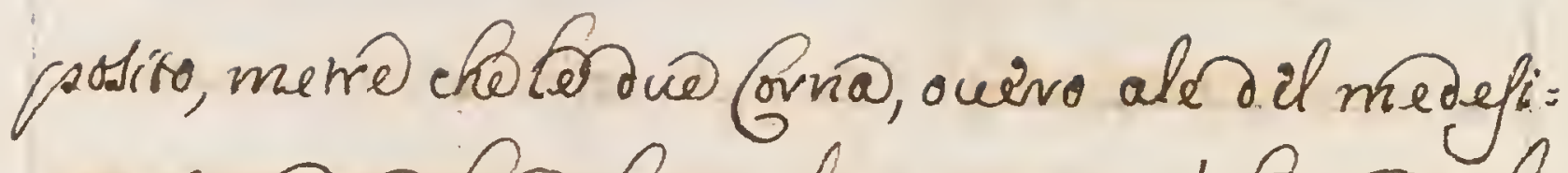
mo son aculéco, le quali quanrociu' fa cordal

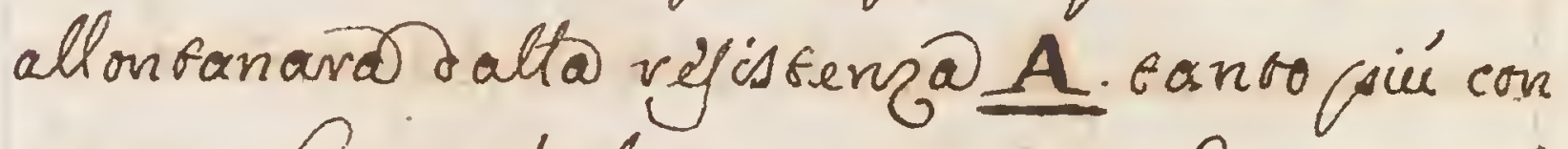
impero Anciará it davdo, menoroche ef endo Ĺ. Aveo un@figuro, aquato como qui alfpryso ui Timostro contiene un@ carter Jella civeorferem: zädeliveolo ct unaLine@ velta comb B.C.Dri= manendo in quisfoforma Borere ali risosareb: - evo quasi nella foro quiebe, ma.re Aa wiolenza

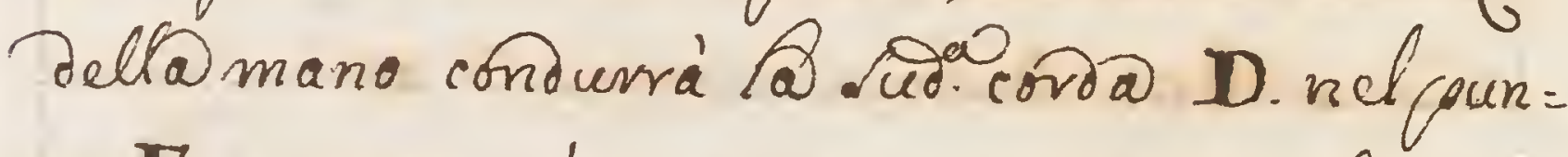
so $\mathbf{E}$ acquiftara' maigion nigore attefo cho $\mathrm{ci}=$

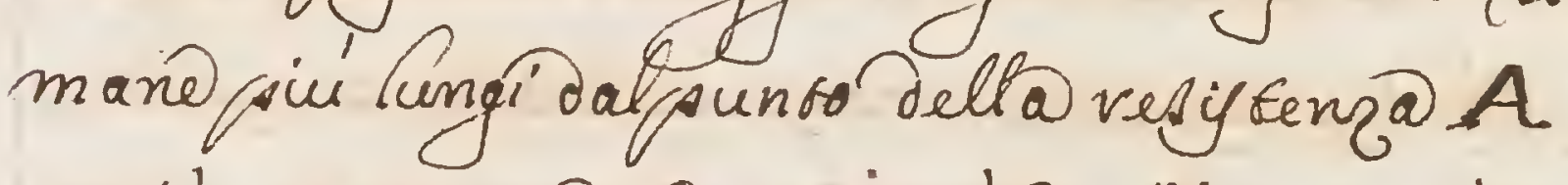
e cosí parimented Davitiva'da E esavá con:

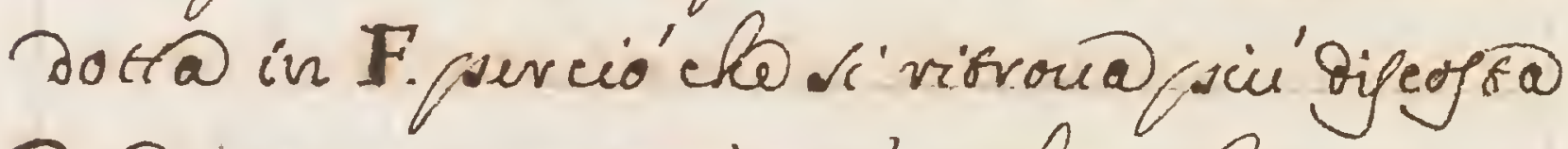

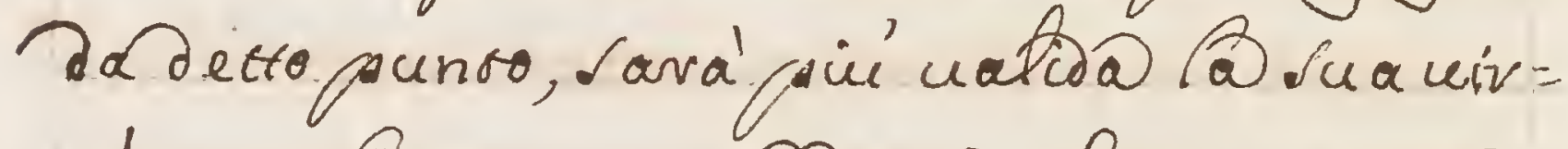

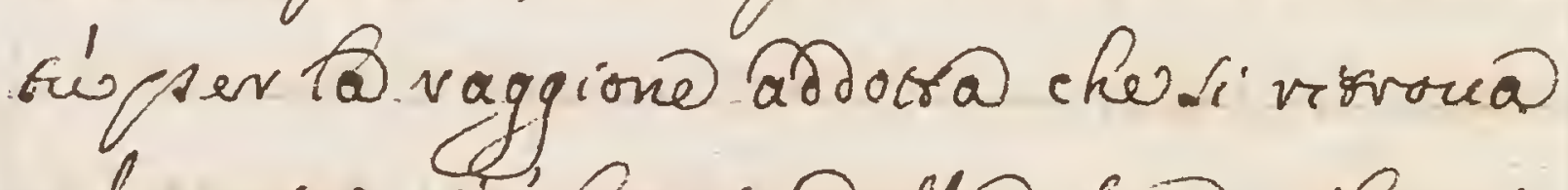
nel centro guiforzaco della. kia cuibuari: one.

Pers 


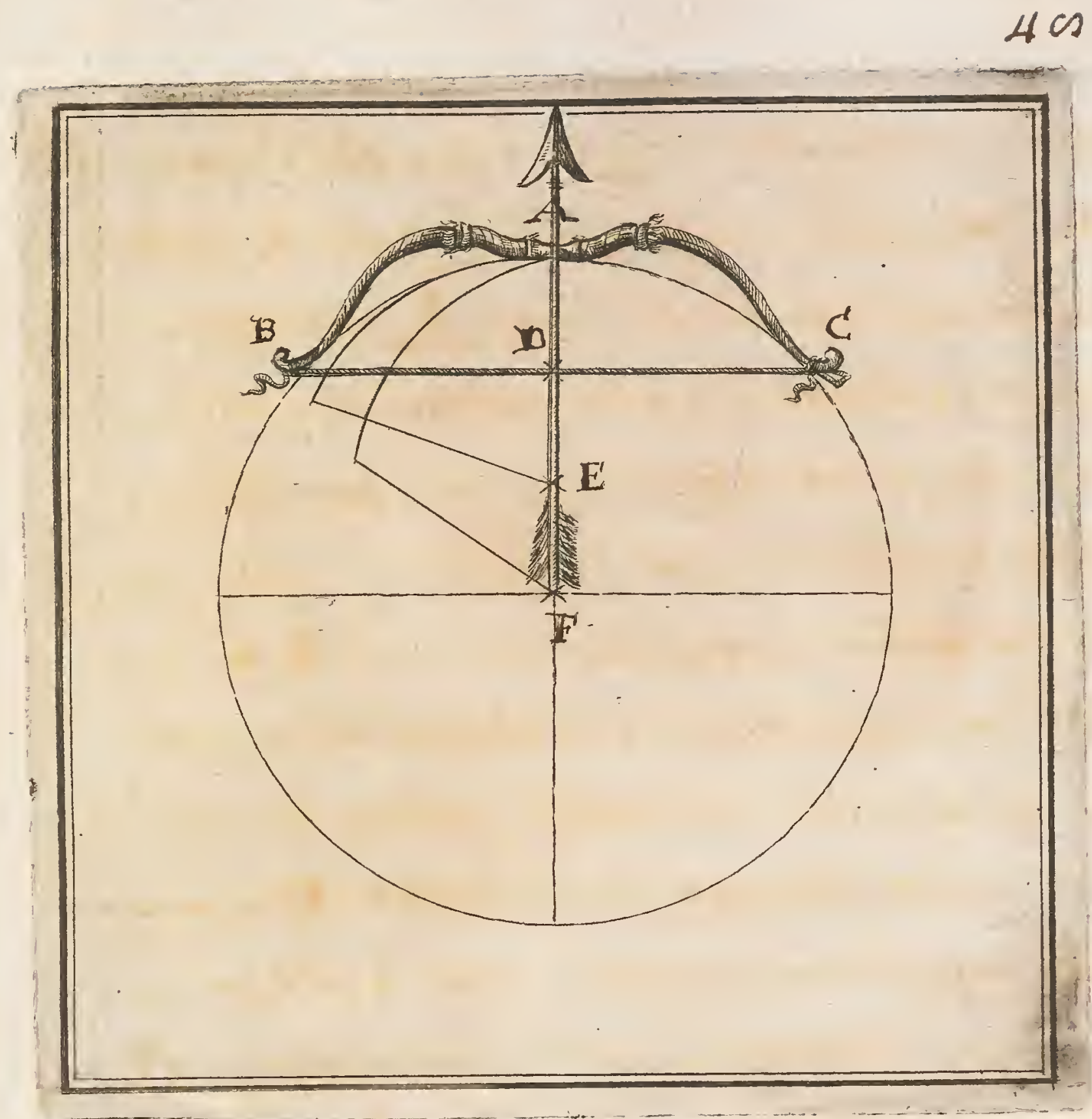





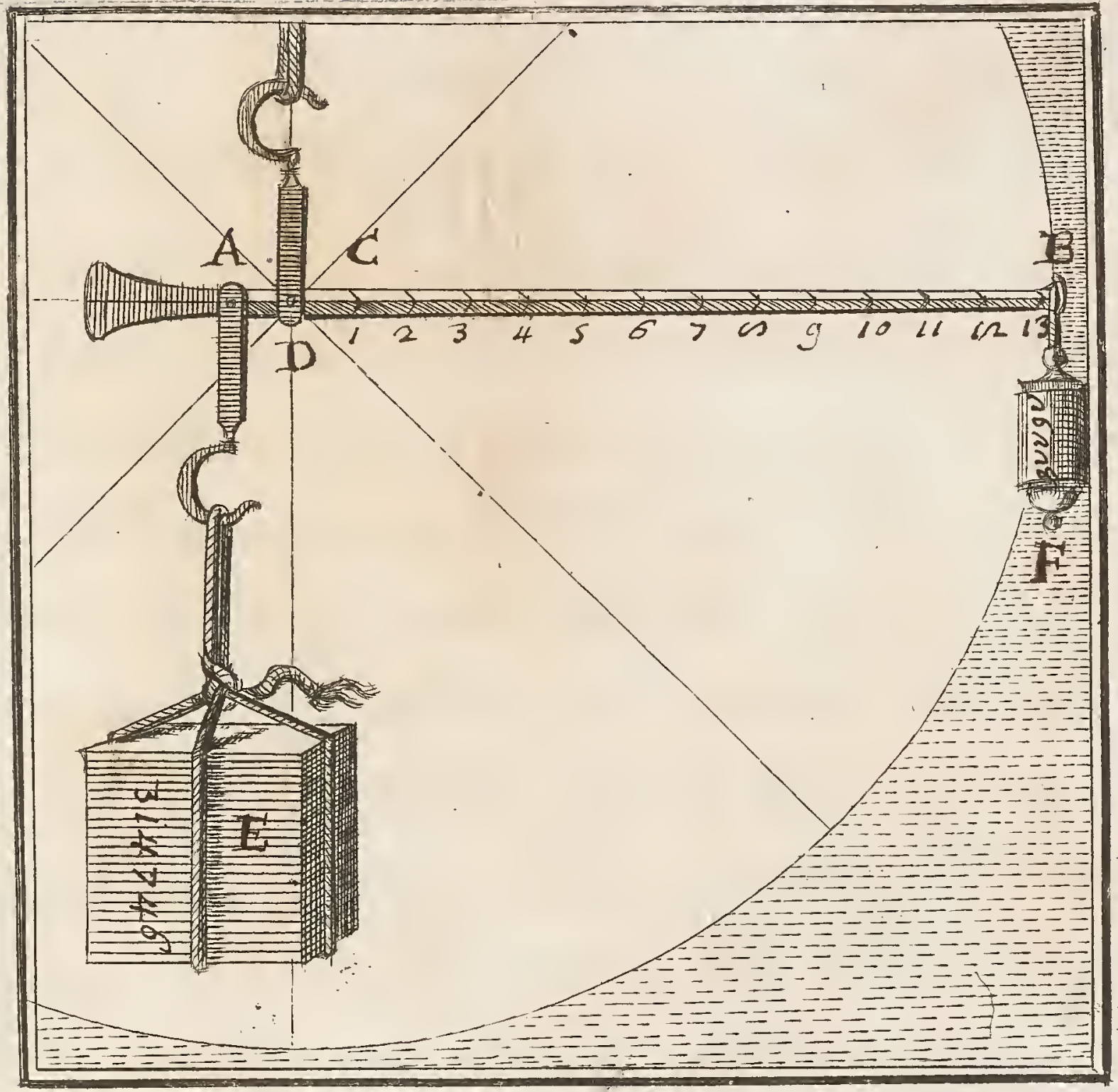



so

Ser quelli, Rquali non uolef $=$ - evo vimanen sentuasida ciócho s'é as posto mi acuingo à farte confejiaro con "uídenjardeltaqui.ruva edposfabdemo. - sratione, cho arra hi necefsitá che A vobe Dellamachina, che ho composob vacchic =

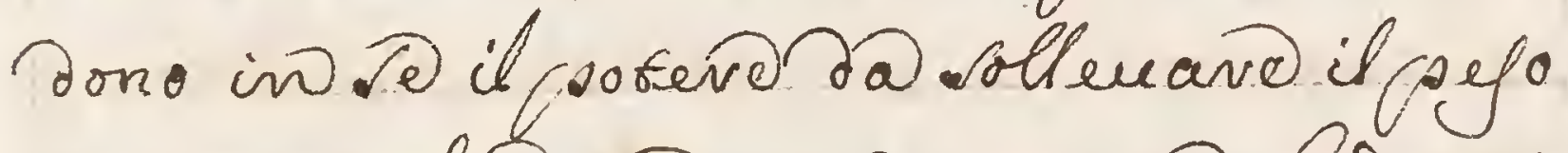
prestrittolidatânasfarevalasicui adta.ia.B.Briujin ivericipark: iguali,e collocari. i. cior colinella let = feva D. Dies che if seso Evilifitiecinto quat = fovdicimila retecento quavancadi, chejg Capunto avebbe quillo della fonnota noi mopossa, a quate appera in A.Cor senerta ingieyso equilibor vichioderà, che Siconga un equiualente grauame pa= riminterditso fucento quattordicimila setiecento quarantadii appess ino C. 
e codi rimarvébs equilisicata non co:

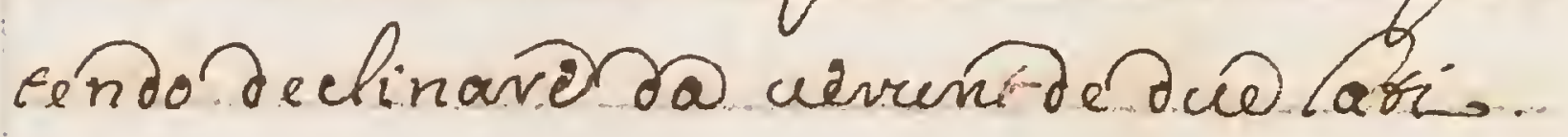

A.C a'cagione peveftenci un equatijsan: zुow Dad A combaD e C e quan = rociu' Pequipondodello.ratera nella letevoF.jojeostavio dal cintuo D fan = ro magioum. Licemarebre fofarica in Douer olleuard il cesso E à cagione, che now habi fijogno sev cisvouardi nell'estre = mitra' dell' astá cke del ualồ D. Cíno craps. in civea wer confraporsi alle तif $344>46$ che rivifrouno ino $\mathbf{E}$

Io ho formaro 1 mialua

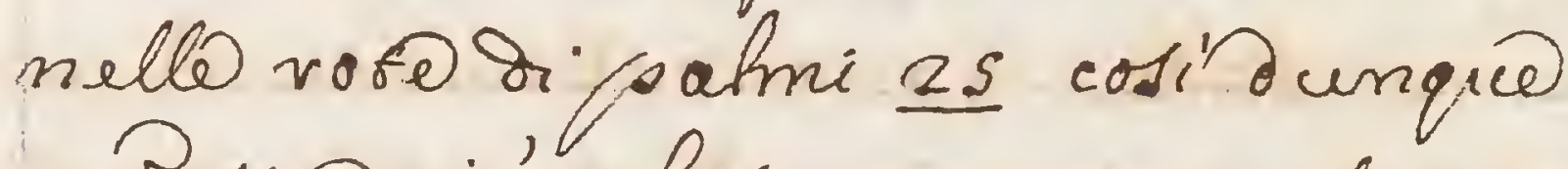
6 Pefseropiuprolyso in vipetervie il gia Hoie: gatoci posverevegolarmi con Geropvafovita pro:

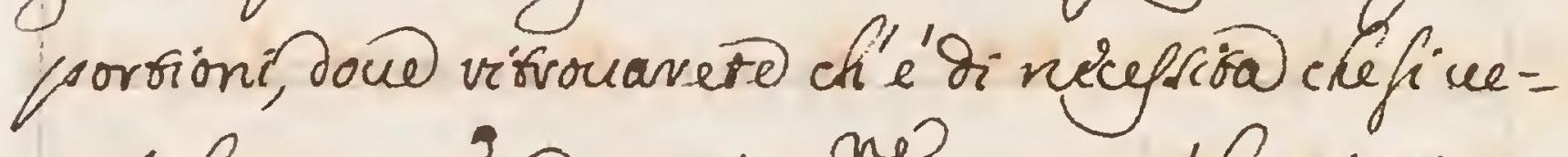

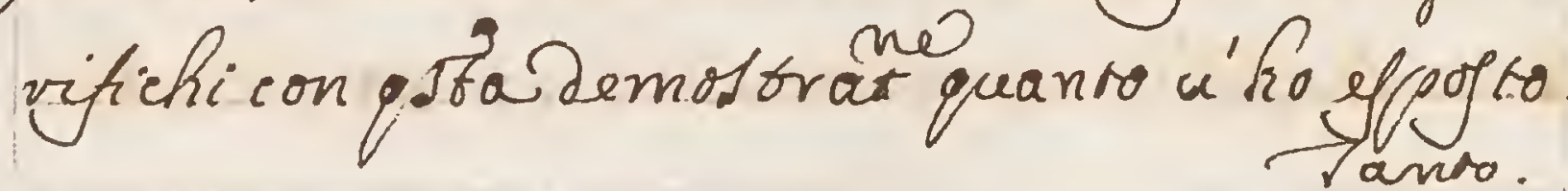




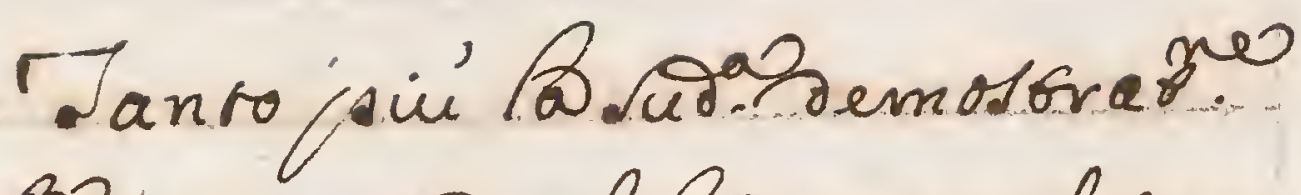
conlSeginuenutanelfarranonel formo de suboil fif. poparincipis matevin forma et uacuo cien concatidaba, owil medes:mo anche ingegnotamboratraldalta: tero, edel cio guedro equilibrio, e ar fon = Danell@propsisione uigetimanonabel

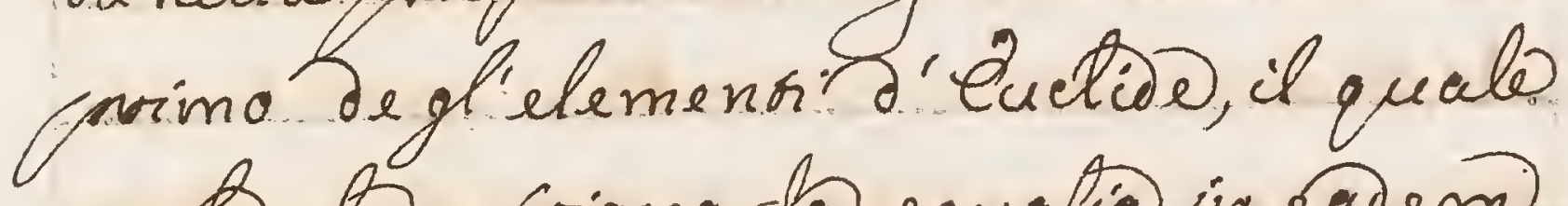

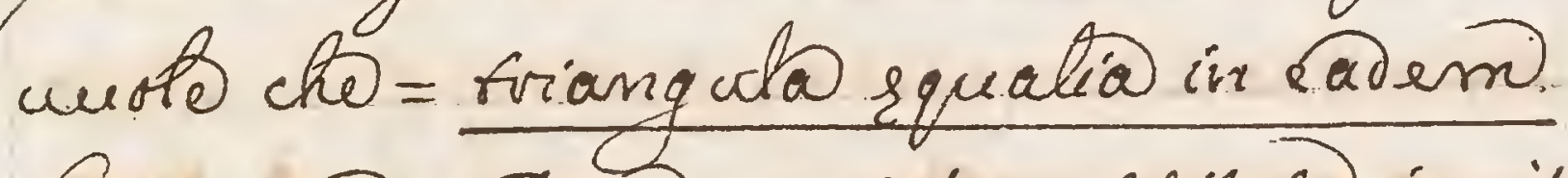
Sasi, et ad eapeno partes constifuta in eif dem quoque sunt pavarellely = onde il.

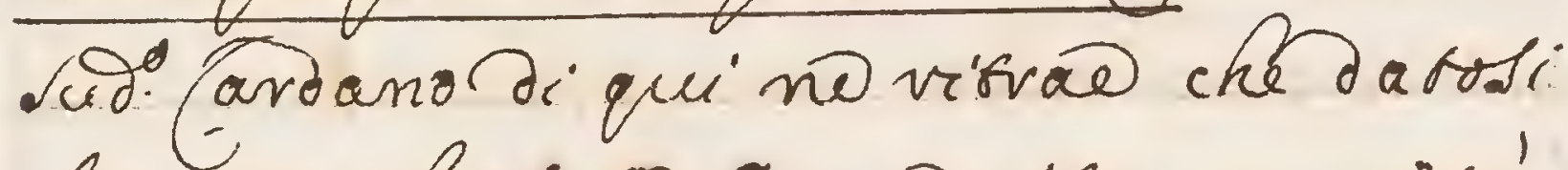
il friangolo A.B.C. Soivifo pen mera's il aro A.B nel founto D condotraña Line@suntegiata in $\mathbf{E}$ cho Lara' pava: bella alla linea B.C. A cosi condonta

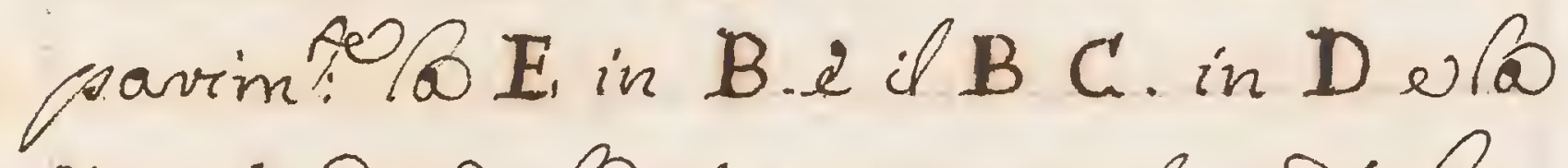
Fin $A$ Dice che aramo infrabi foro 
quelli oriangoli uquali, Tiquali iavan: no costifuifi Su basi uquali combil tri: angolo.B.C.D. Raventoobabocom= mune al sriangolo B.C.E. Saranno for Oro uguali, d consequentemente Iintersecationi in $\mathrm{G}$ cidaranno il centro dell' equilibiio del triangolo A.B.C. o Serventomi del medesimo perminorael@ardano,aivo, chopejto uno sile in G. I firangolo A.B.C. in nullam cofevit coartem inclinaño

Accioche 
52

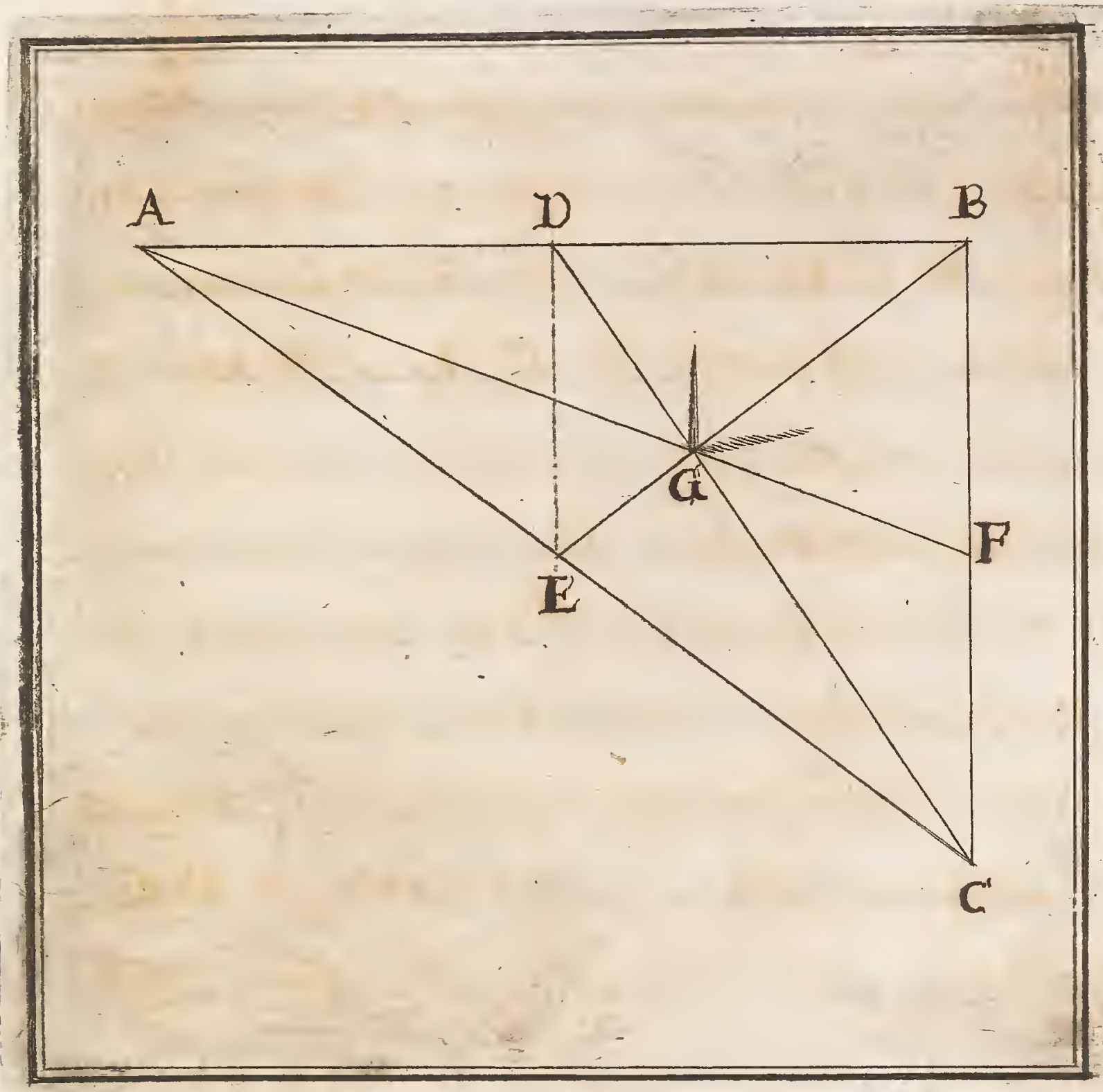



53

Acció che ogn' uno condideri

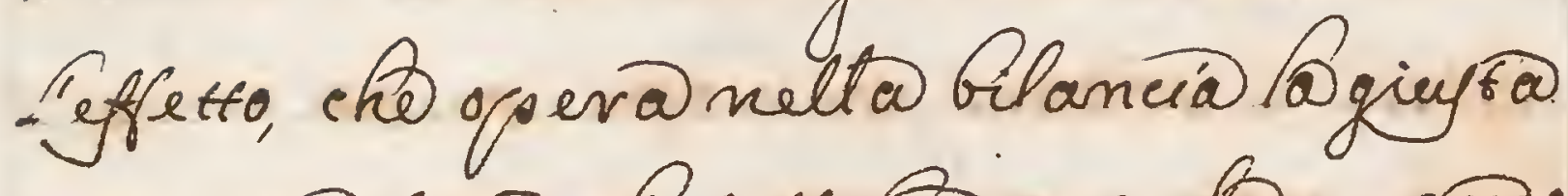
civoportioneseconos.sfelso arrano, che quando iliudie D. sisrouarióin angolo retto al C.A.2 al C.D. Sava' nel suo giug tro equilitrio a' riflefto, the

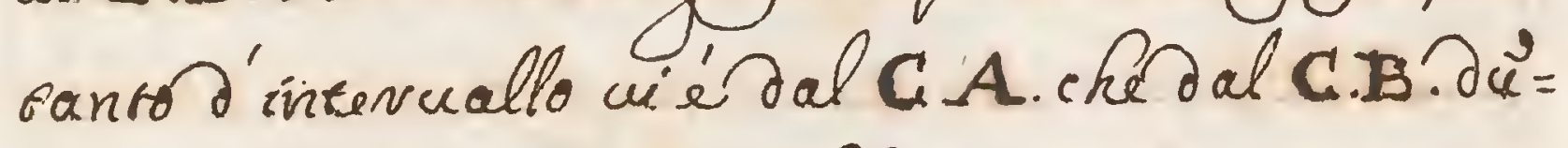
quede il peso proposto di lit. 314746 fustoposto

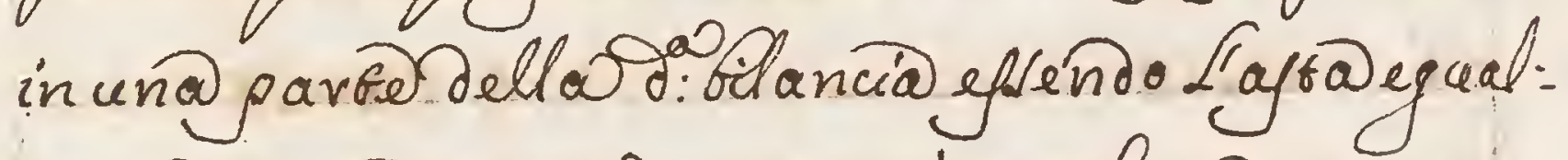

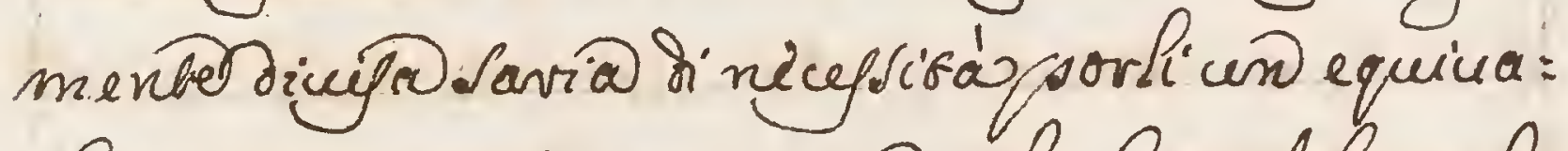
Lexto contrapofo biselo per danti Requilitrio, che - picí da unaparto che dall altro siporrejso un

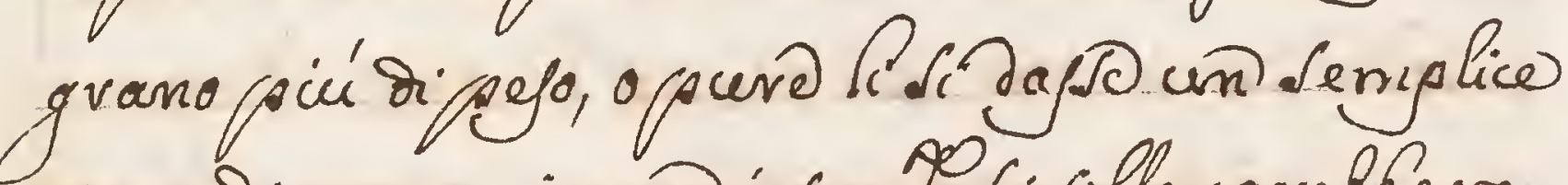
tocco dimano, immediatam? Si ollecuavehbero. Jall'alw@parpobselit: $314>4 \sigma$, e cio pvo =

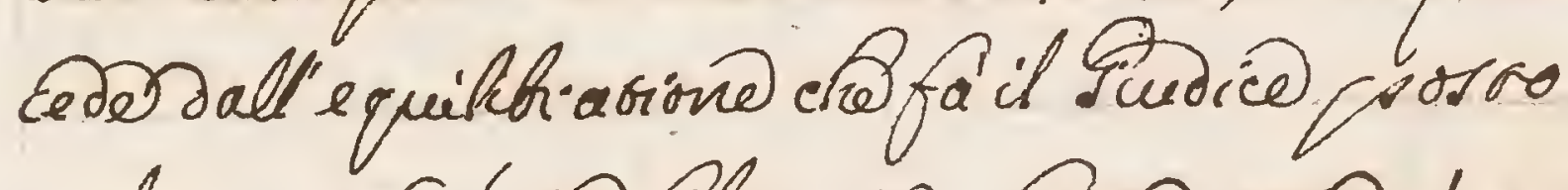

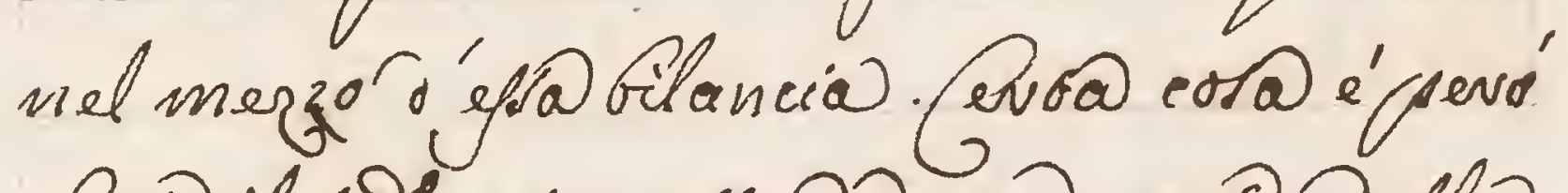

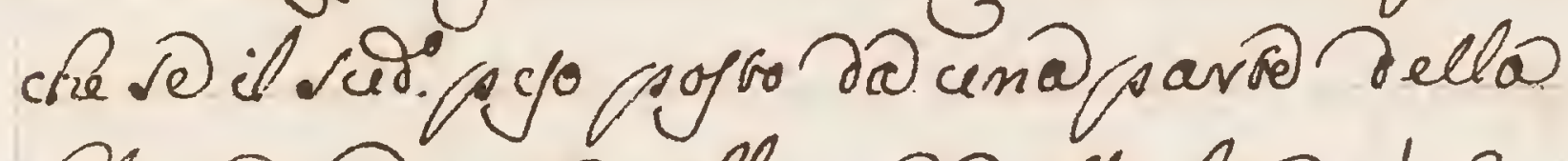

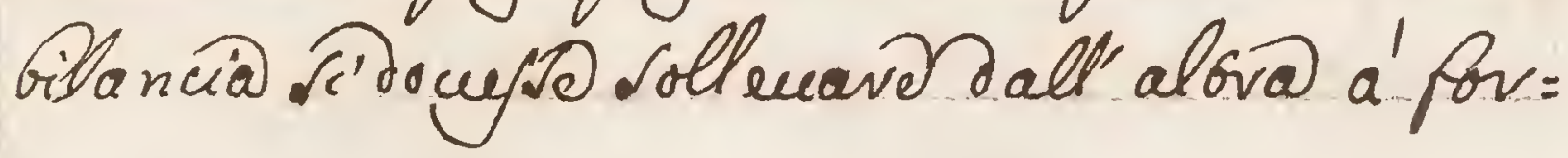


D'Accomini, cico'vi veñdevibbe molto infof = fintis, ovavei pervive quasi impof:

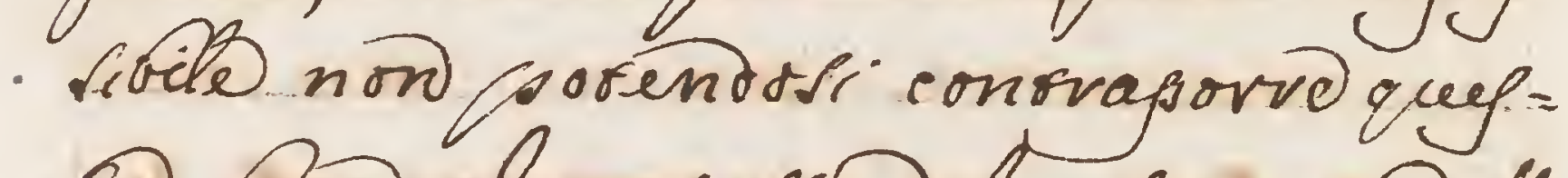

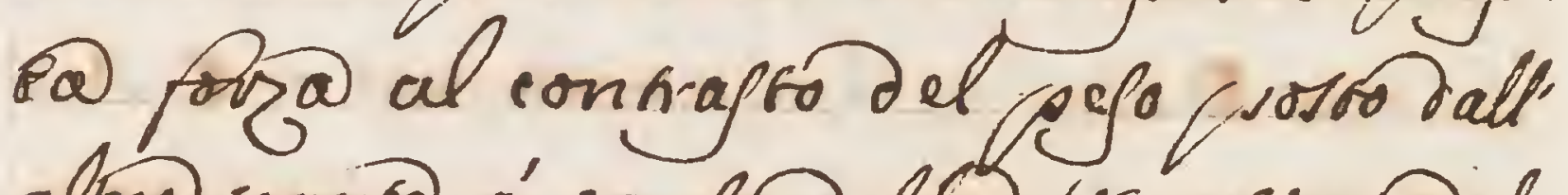
altroparro a' caula dellodiscuatione del Purice collocats in muzo dell. Pfal; Ton cosicsevi auriend daltoroch

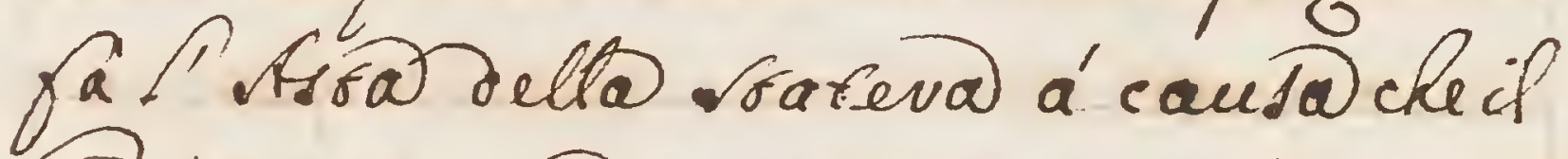
Fivicesirocia quadicissno alla rejea

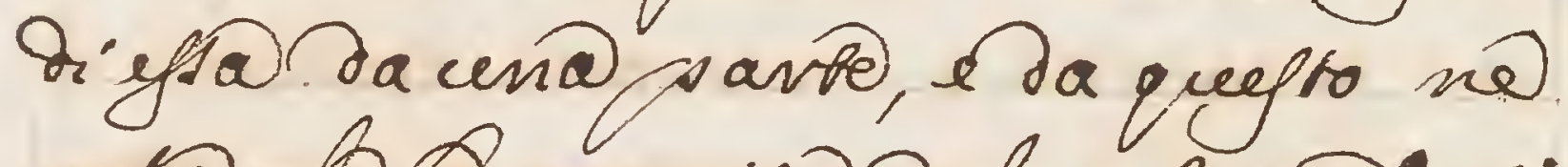

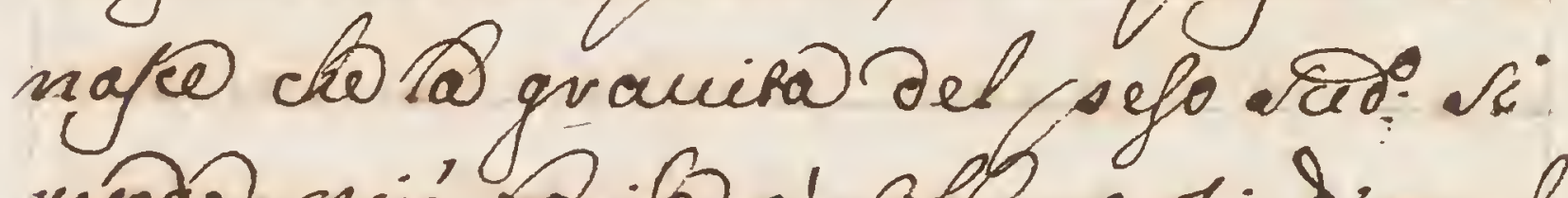
unde cie' acito a' olluardi diquel. Iofi vente posto nell'equiliarationd della bilancia come aedrafti in ardervesto 


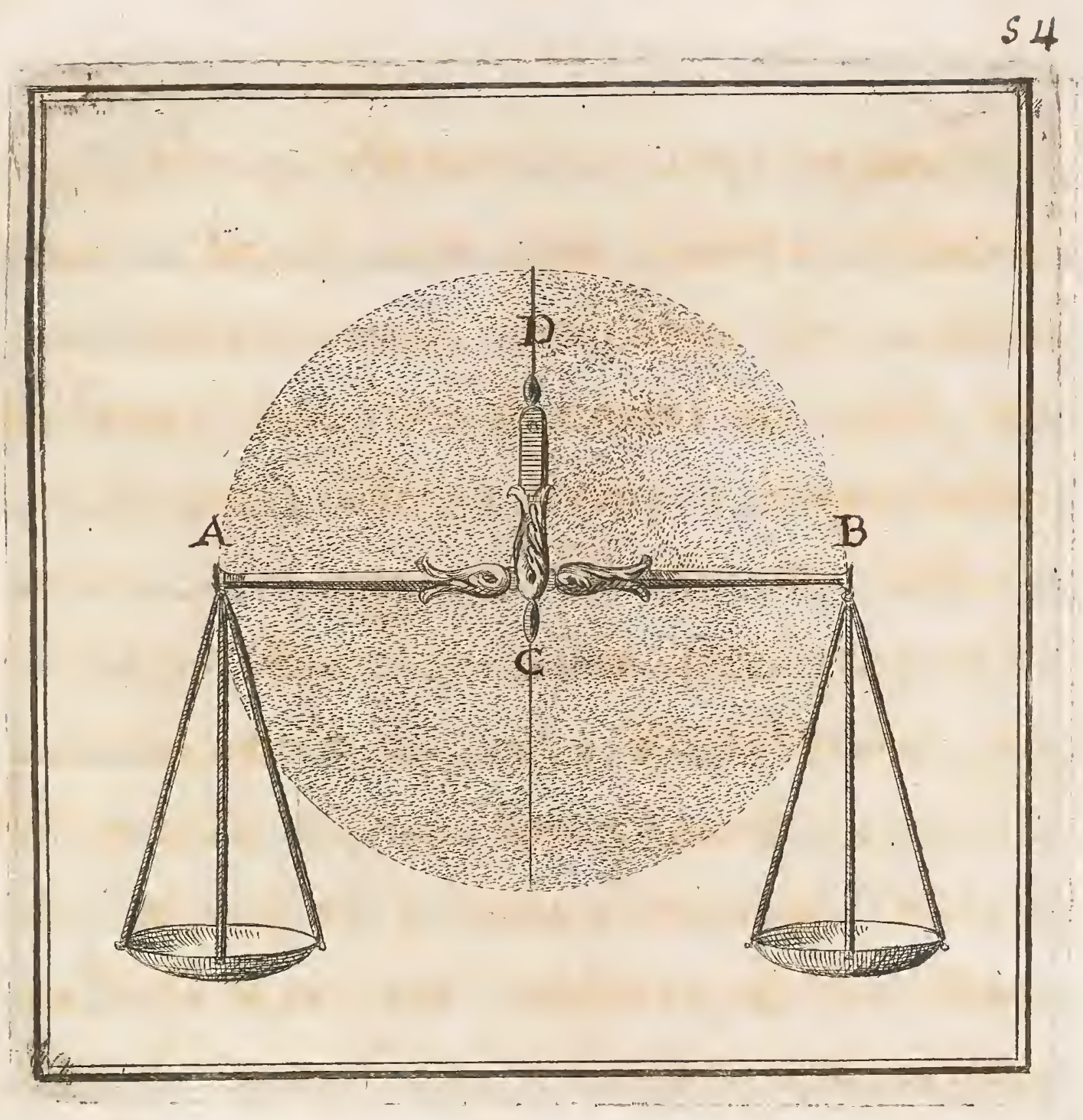



a jequento dimostratione mídá

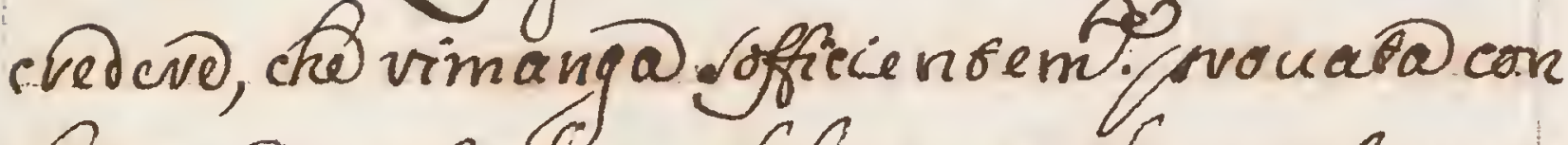

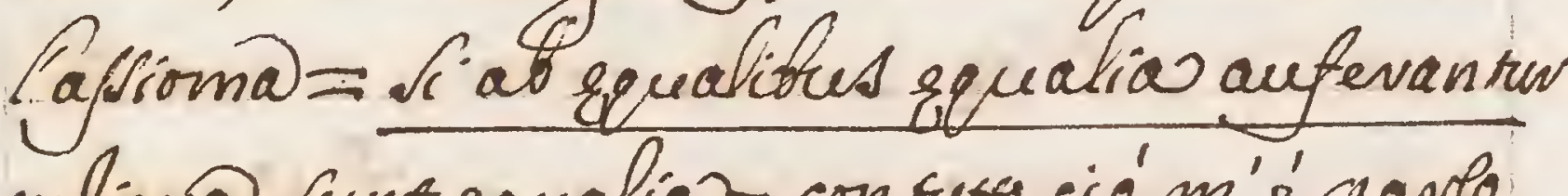
veliqu@duntsqualia =con futs cióm'é payo molts necfaria fefficitionordellamedesima

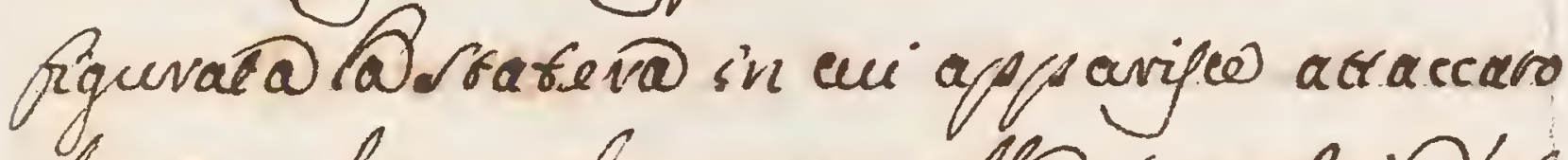

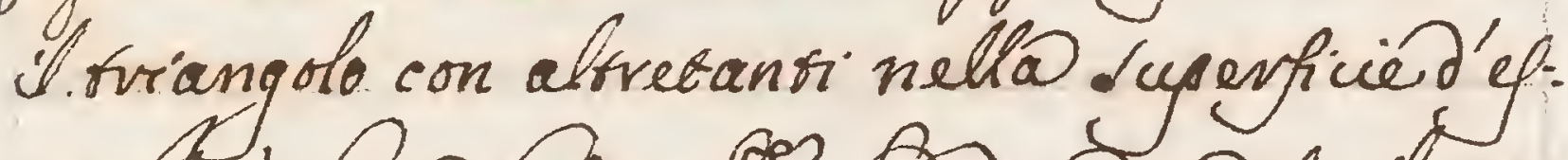

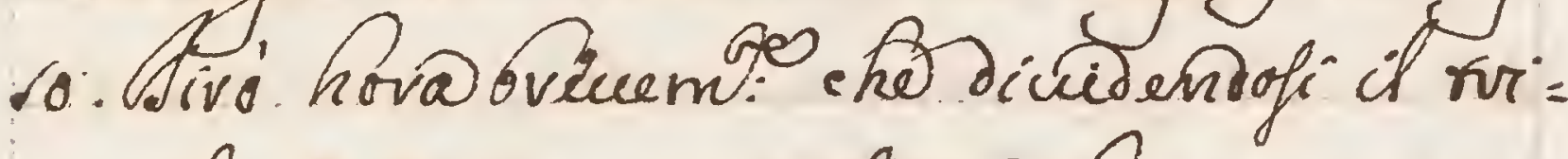

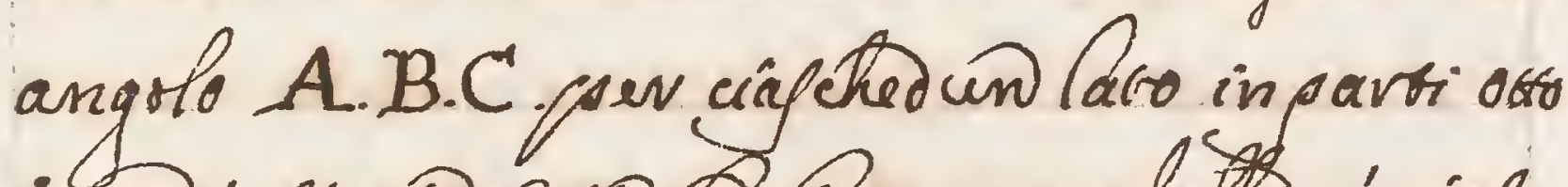
Lindi fivare ficto to line pavalelle a' cial:

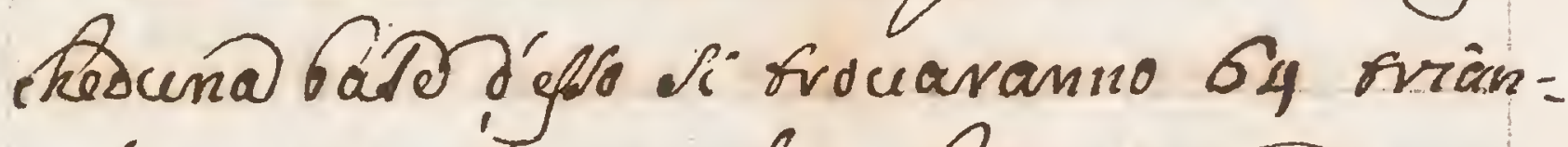

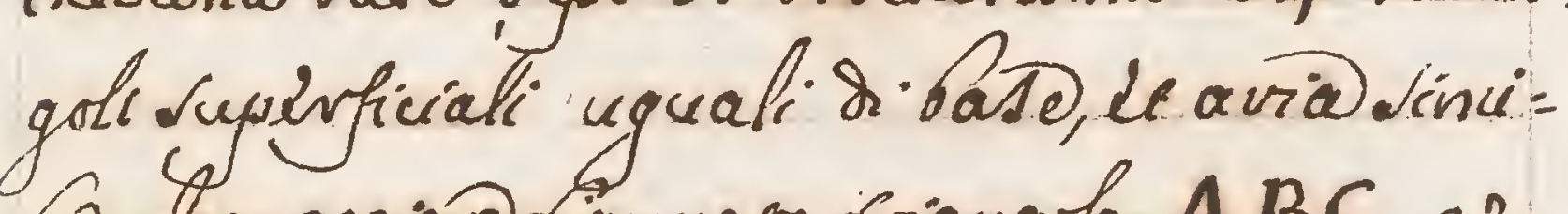
io a maggione figuraro firangolo AB.C.es putunganoo 86 aro $A B$ in D conta mede-

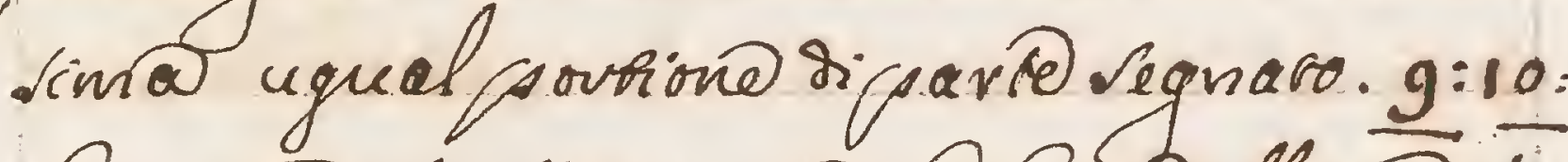

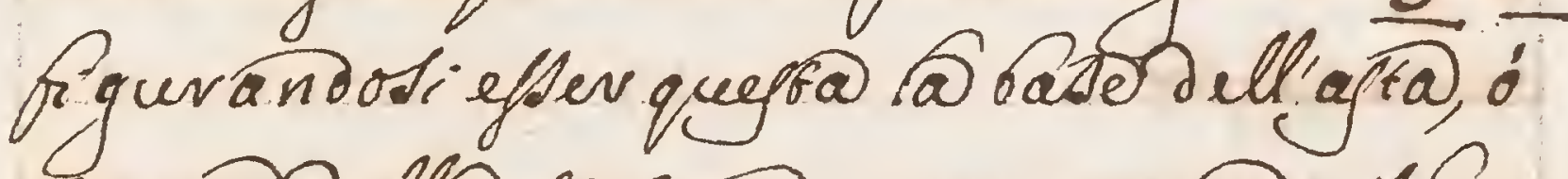
rangardella.frafera, epor fomendo il Tci. Dicenelpunto Segnato g. enell' estremitás 
Jell'Ata A ponindo un equipono proporti:

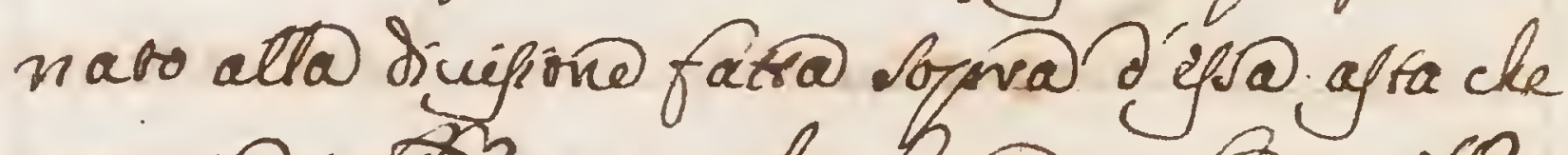

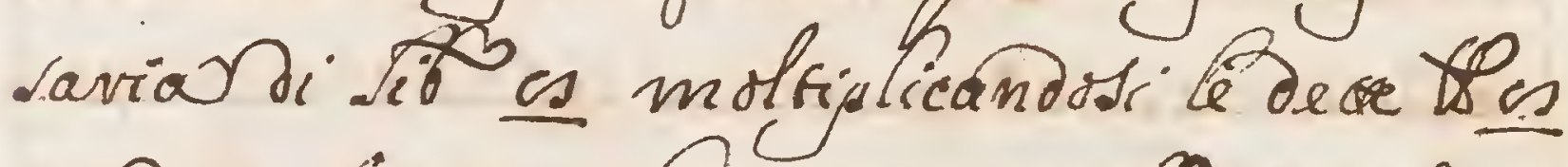
coev es faranno OSommar.t8 64 che Sa:

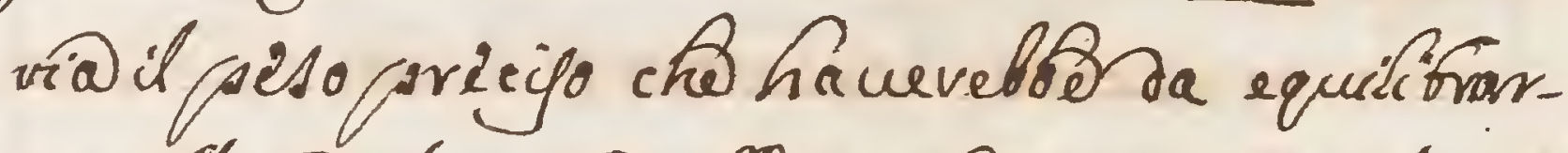

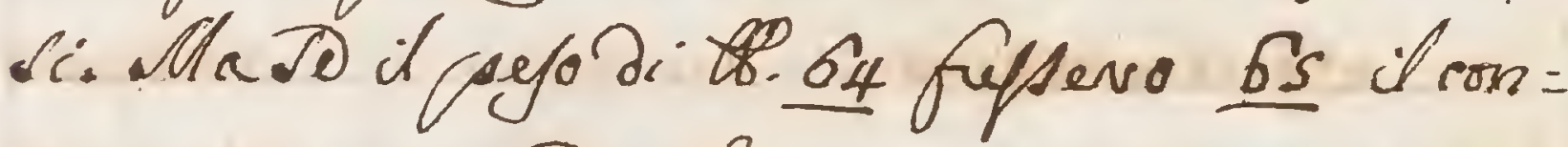
irapostro equiponco cho fivivrociaproportiona =

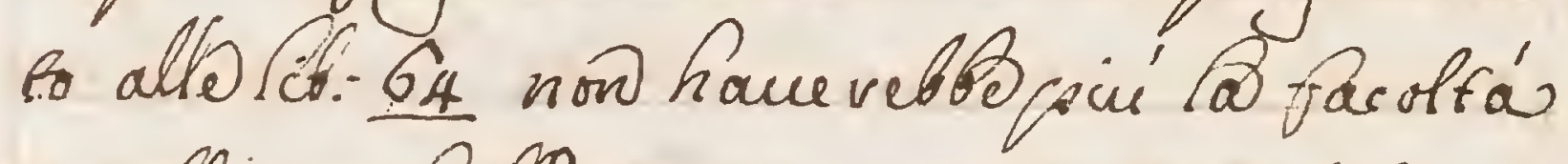
aisollevarole te. Gs opposbe se non S'allonia = naftodalliara A quanro fopso d. Fiogno

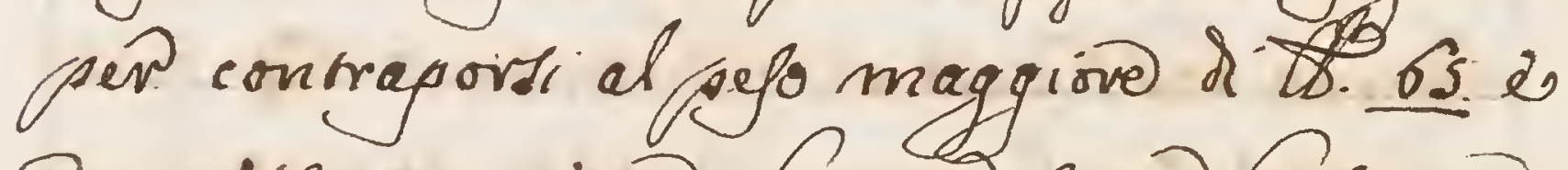
Da quelsto auciente che non butte Sistrateve Sgofsono aggraceave timagion pefo ti:

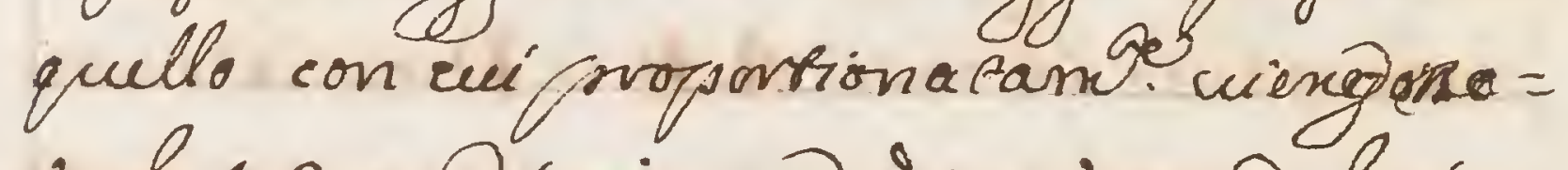
wholate ne aipir no dimeno del suo confrapgets 
56

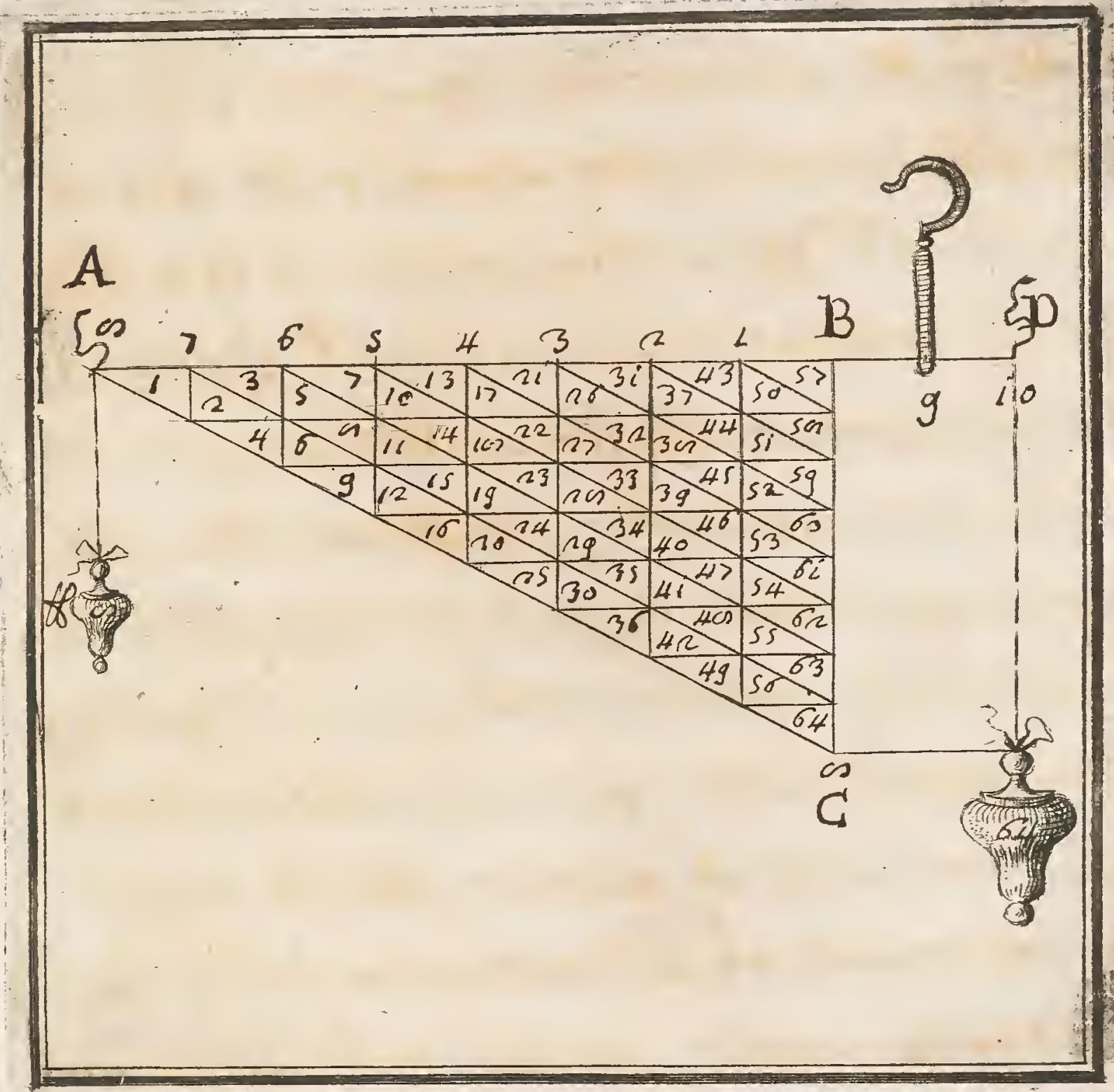



57

Divfarui poi condrovarufin

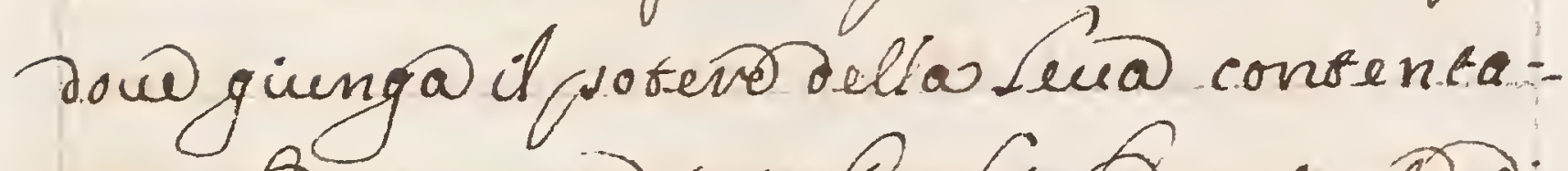

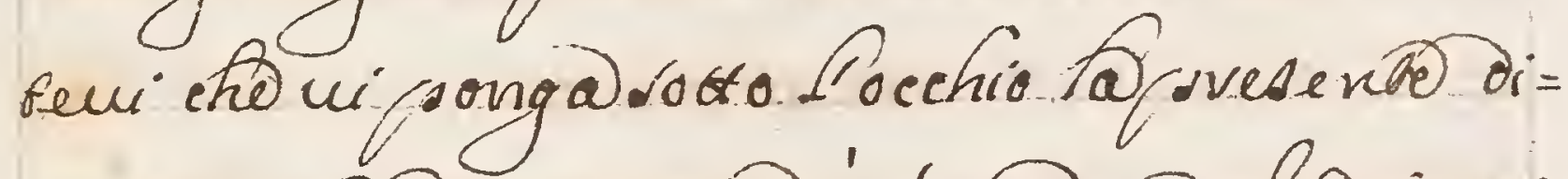
mofrationo da uimido' á ivedero chovimar:

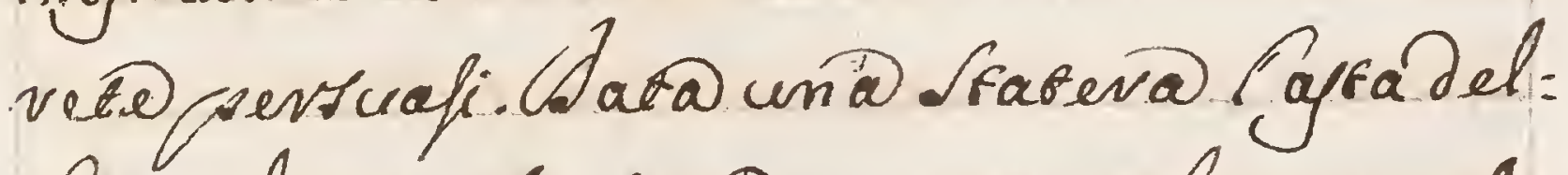
la quato viá A. B to appeso nel punbo $A$. afoloñD.er E la quatono cifiquriamo. Di. Slleuaro, es immaginamoseta sipefo. S:C8 13 e cosic sosietse ancherivo fresici milionidi Litos, es Samajo bilanciand con

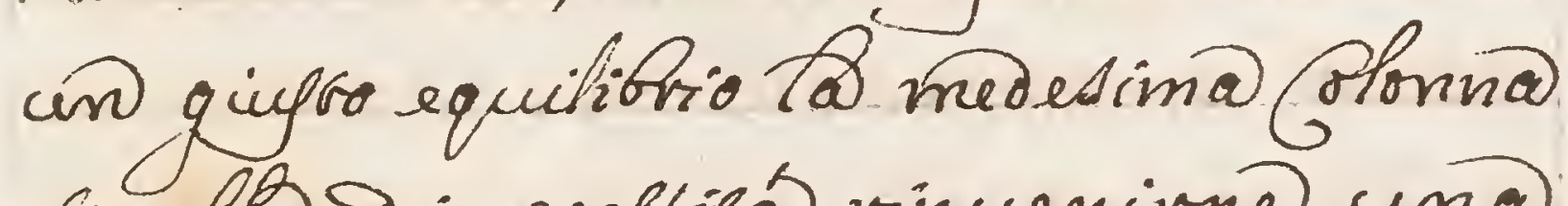
- avebbs gi necefictob vinuenivne una J'unaconsinilefproporsione, epelo, ó ce = vam. un pefo equicalense, chosoccum: betse all'officio dell'equipondo, o vaceman = Jarlonella Lexera F chenon u'discor:

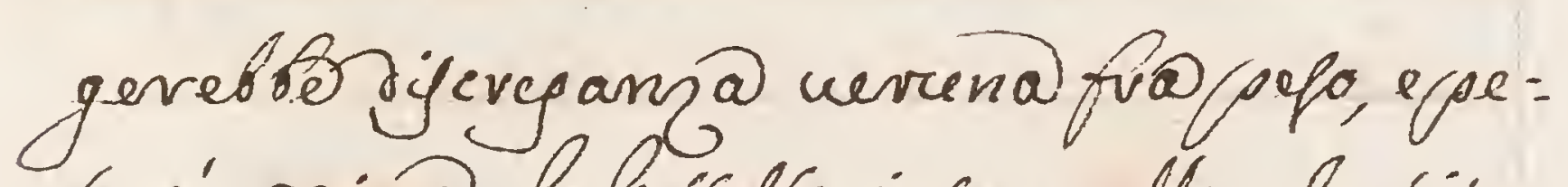
to a'cagione chelosiffo intervallo chesima = 


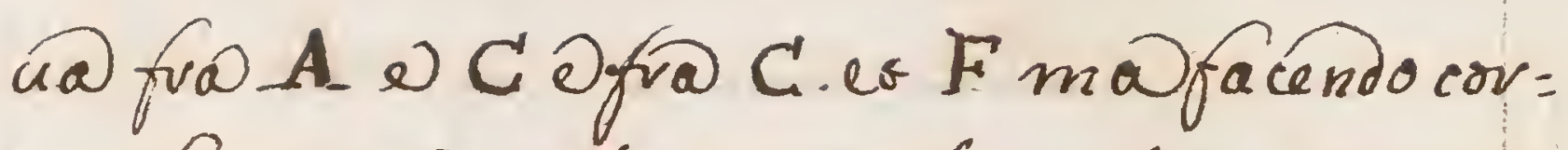
vero. Pequigondo G Souvail num. i injtan = i al medesimo non glivimarrefor cho

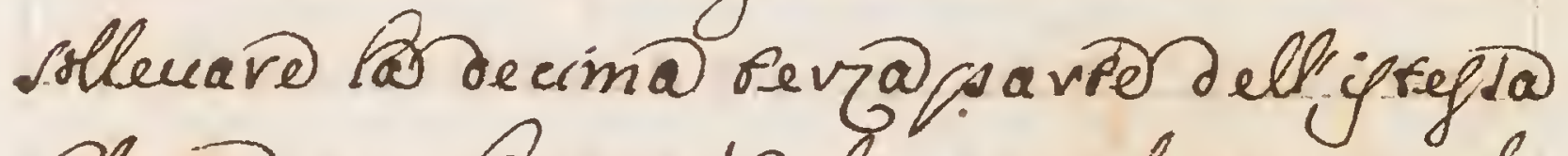
olonnasev@uivu'del triangolo versangolo

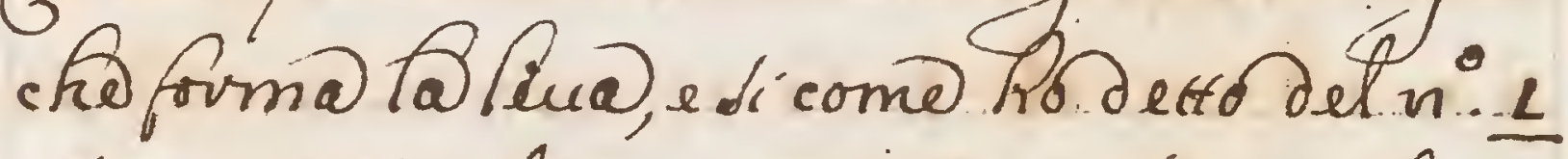

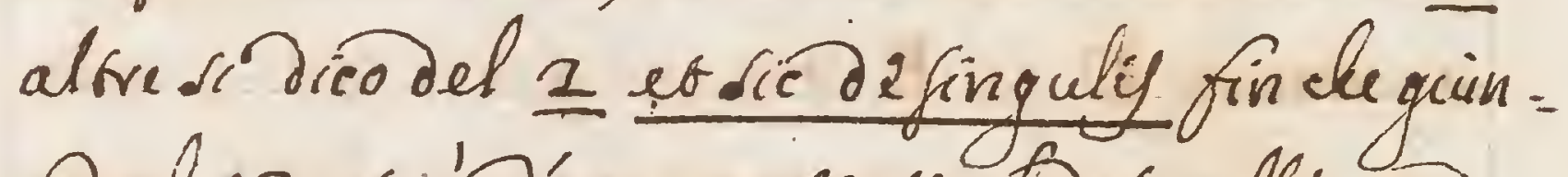
ga al 13 ef e' J'amertivsi che all alva Pequipondo di componefordicin pejopoce

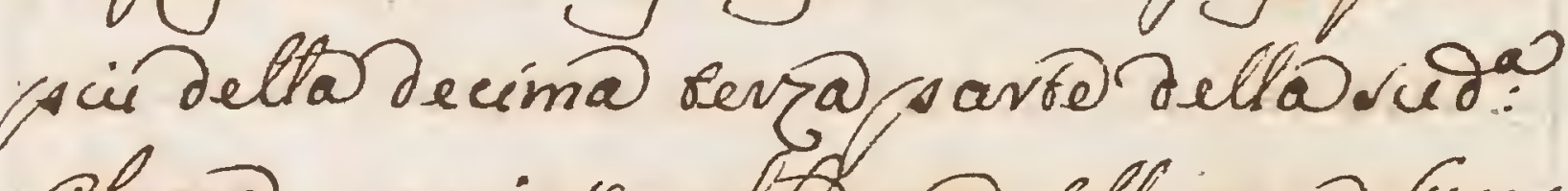

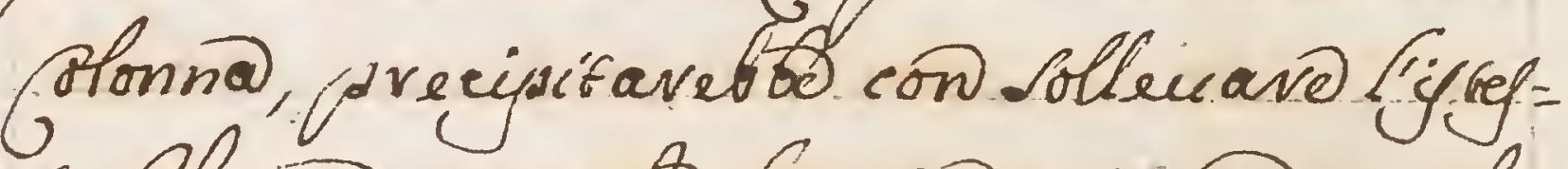

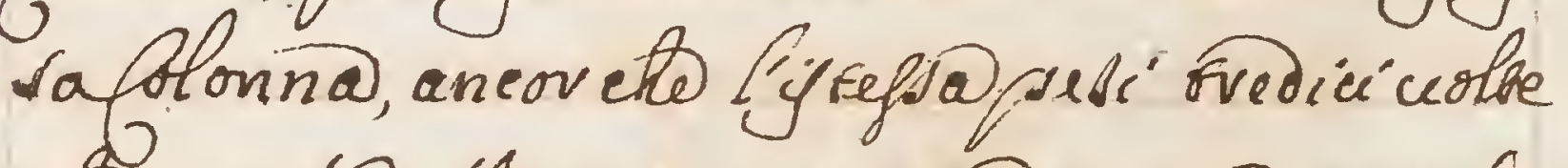
at dicu'delfigrefto equipoño, m@percke ogn'uno quando uogtia potra vieonof cer ne Peffetto in ogni satera, perció now mi bilungo a farno una picú Lingâbeferit = fione 


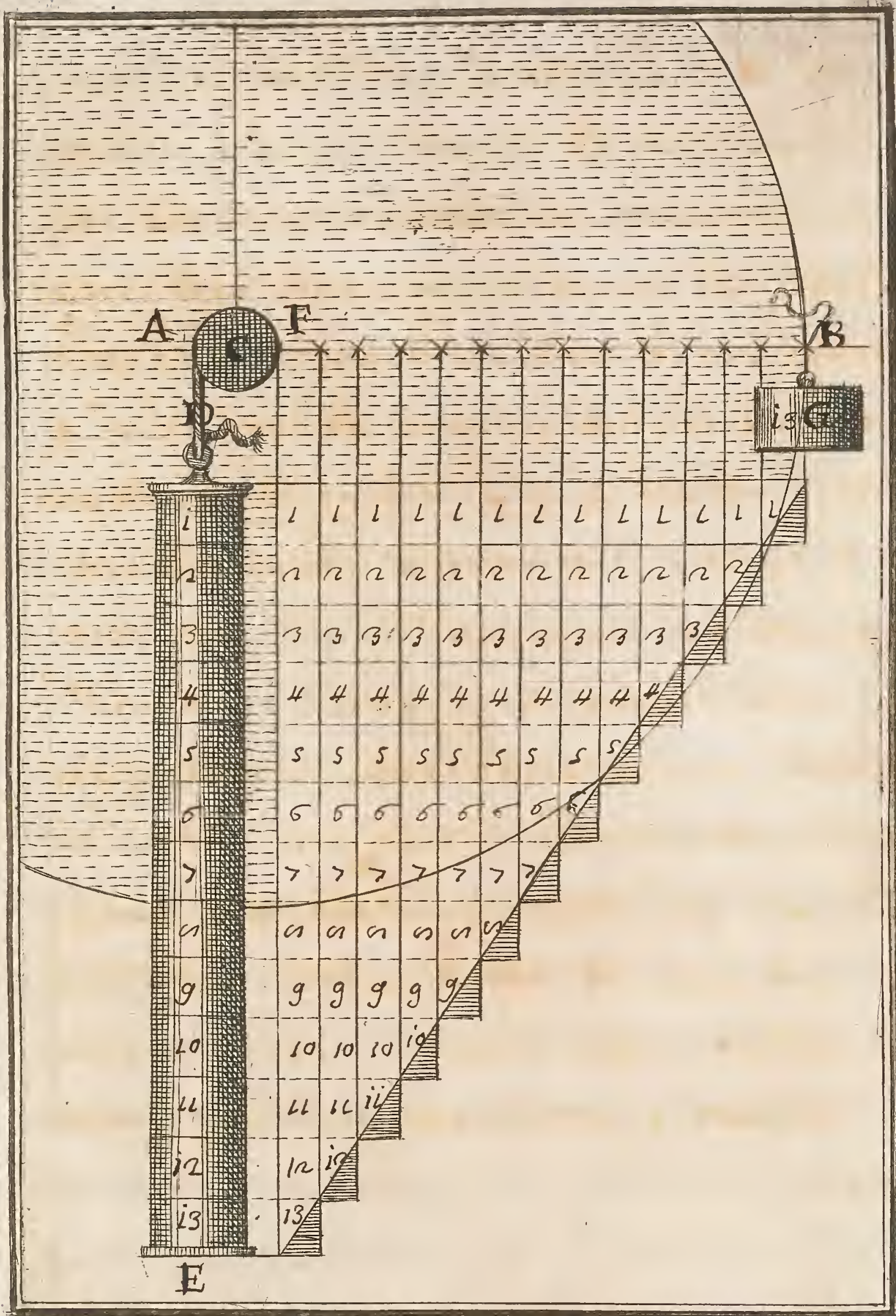



sg

ontadimofrasione che vimiva: vere qui Loto esprefsani con Favgano nuou@inuentione pvetendo difar cono: survilollieu choproum im hurmo quen. re gici' Stallontanava' dal centro or. grancita, 0 hico chepstro un Vomo alla sanga, cho Ruovi ovizonsalm: nelta lez fera I now ava' capace che hi muoueno ifcego del triangolo numevo L ma crice = uinda pouvete PVomo nella Léte-

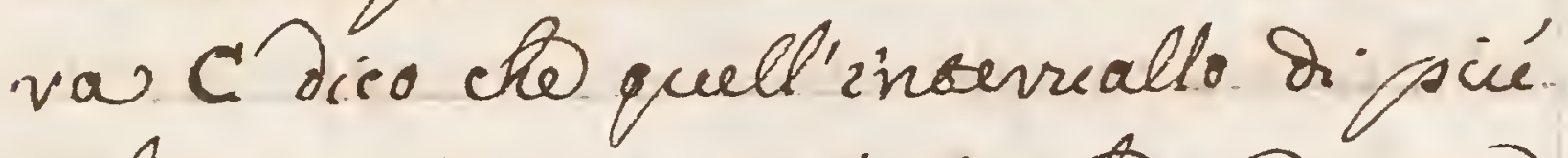
Dal centro Sigrauisa' A gídona una ralqual for ac muoceers con to medelima agilisás quatro delli meresimi rviangoli nel junto $C$ cono ne muocepto uno. nel cunto B per vendenci maggiovm: appagari, $\omega^{\circ}$ covego a' condidevave $a$ de $=$ guente demofratione che come hibleto 
ponina Fomonel funts B cevroce nion muoced, che uno do $\sqrt{u}$ etrit triango:

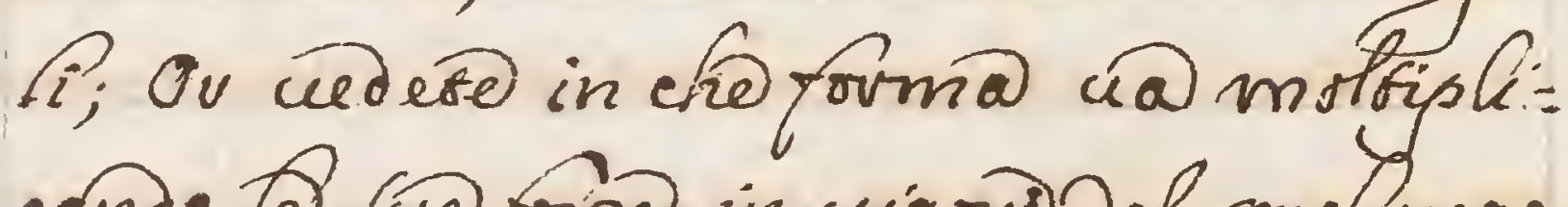
cando Profone in cuigond del protunga: mento della Léco, mento. Se correto Gomo nel founro C'. I numivanas $i$ Sudeti friangoli, vinuervite cho ne muro: w quatoro, nel D nowe, nell $E$ Sedici nell $F$ unfieinquo, stre ser conse = guenzan no najeer shosto nell'eshremi= Fa' Jellastanga, como nel maggión sfor: ¿che puo favo @ lür, fo ancho to forng cho ova fono cier aivui. Sie'bet=

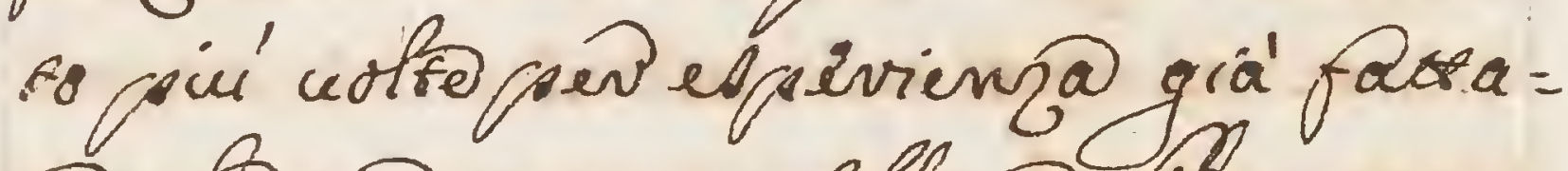
no che cen argano bllecia lit. c0000 D vipavife to 20000 in Aedici Vomi ni, Iiquali ono a'. Sufficienzaperta :

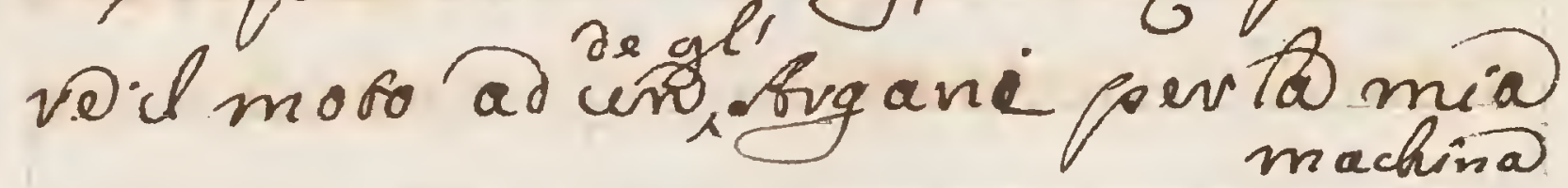


60

Machinano roccavebô coev ciafcheoun Voino lib. I sso centa cos' è che Pim = maginartelo che im Vomo solo poreformo.

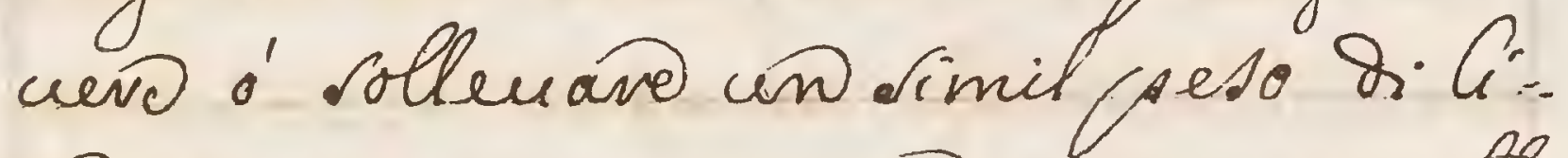
Bî aucento cinquanta, qusto larebre corne conjidevave un impolsibile, non. rfans ció quepto e'un mivacolo viferba= so dofarisi tolo alla reua

Vi ho espurefio a nche of pigu:

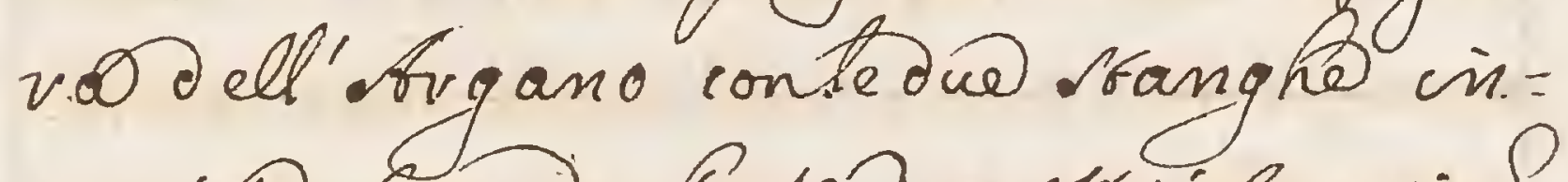
vrociate seondo Pruso de notrit pempice Dalla sucsente pofrete bin olsenuari

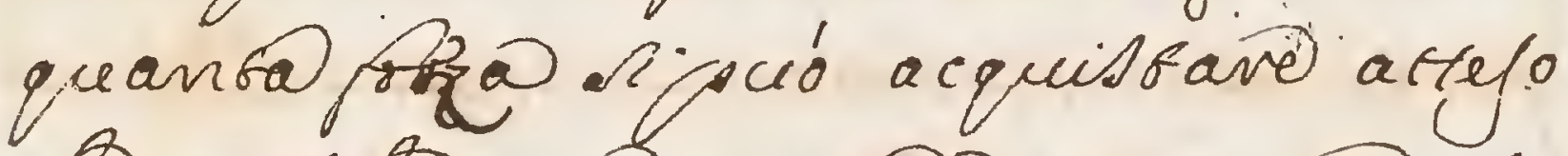

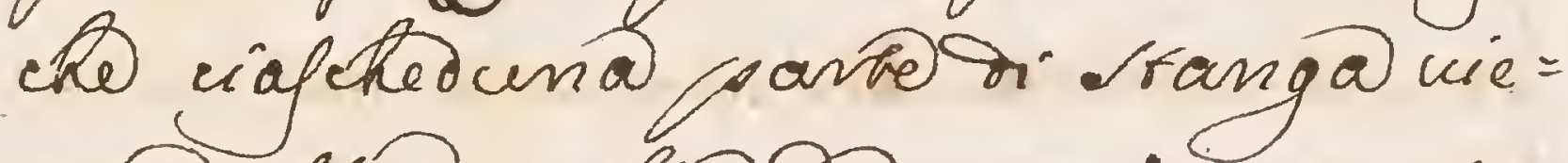
ne ad efsev molsaDa mumiro quatro Vomini, cho in outio to quatso adeo. Fo parbi ascend ono al numers 2.16 ma'conunariflefione che ruok 
quatro Li OetwiNomini ono potoi pert

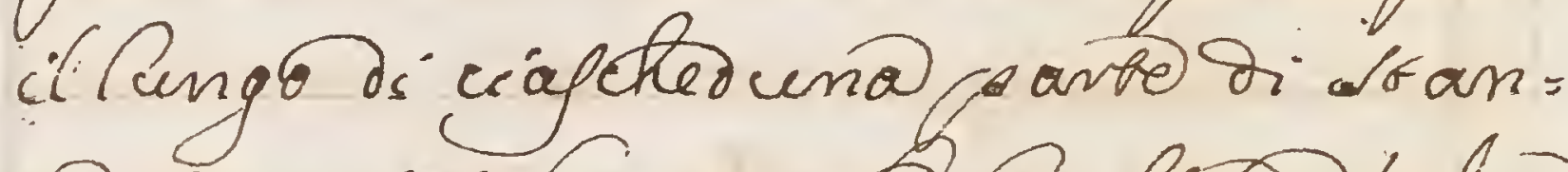
ga, J puessi hanno, to facolba 'alza ve 20000 lifo más so ofseruavab axtecedenter dimolfratione, quanto maq.

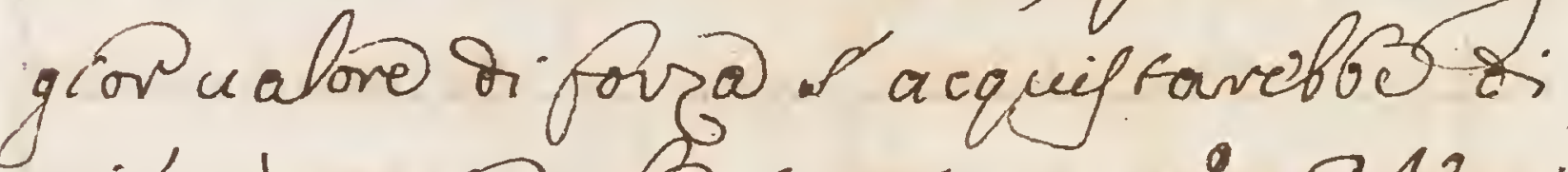
Gié a'causa che retrinum:16Vominisivisruanonella civconfervinso dello vecora dell' afugano, es fuor 'S' una unita courifeondenza diforbung = no a' Voll cuavo dopsiamento el preffif = - peso con Timerómino 2 argani di quilli che faviano hifigeqno indicavo contalcueva B 
$\sigma L$

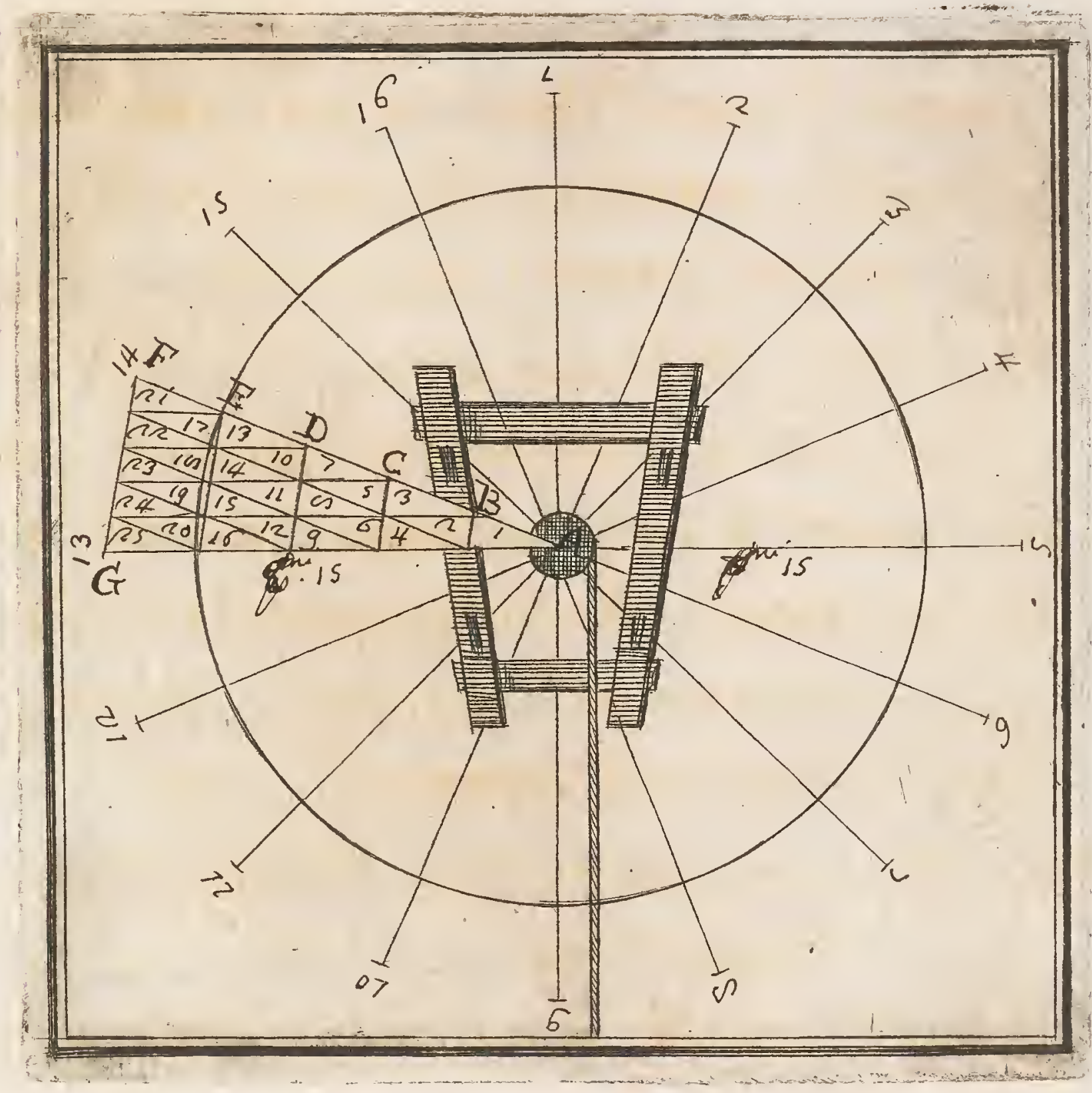




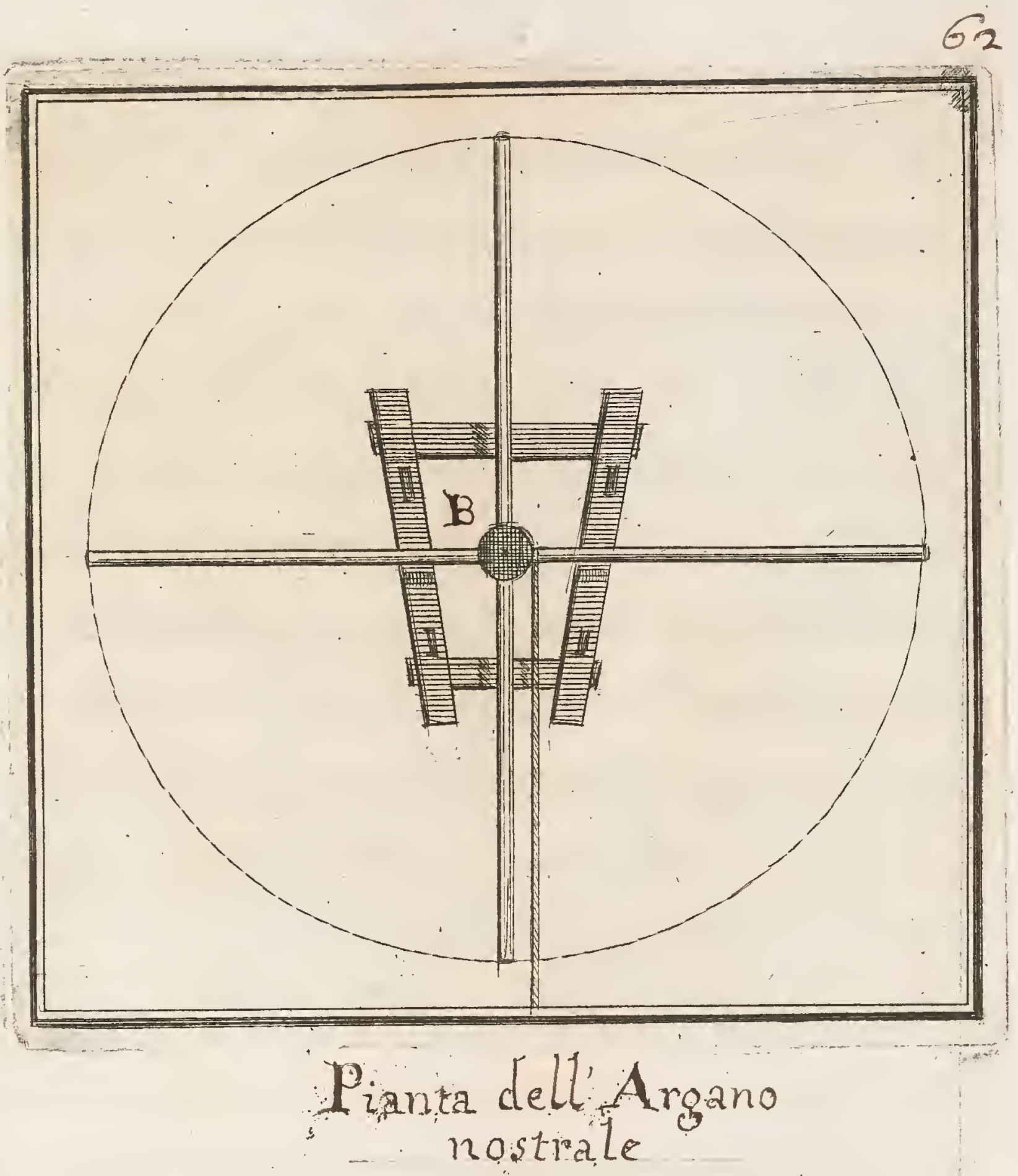


63

E perv repilogaro il ualove or quistsourahichiaratoryoforabio. ni jivó che quanto sić siforolongaranko

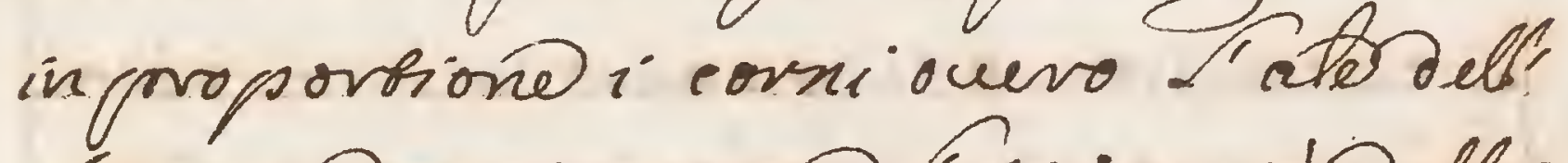
Greo pes consequenja Fformitás delle medsime fanro pié vervanco a'difeof = ranfiral suo primo censio bivibuationo Rallova ava'magyiormiv uation $B$ u'vri, che Lessinge, senfo nifuragli C'augumentavebo Dimpulfo

Om'anche 'equipondo, o' come cofgarmento wiñ chiamavo Comano,

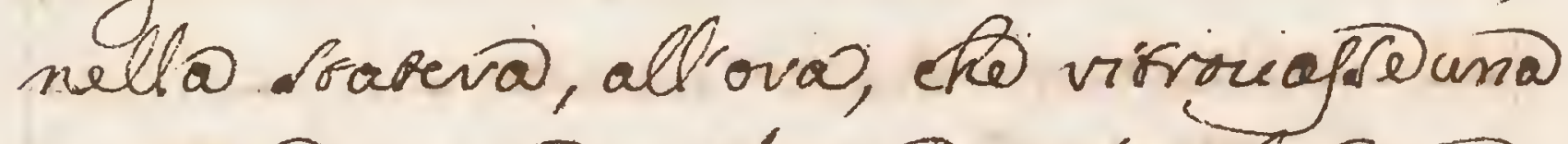
maggior fracso nellatio intimit forma egli maggiormin b allontanands dal =

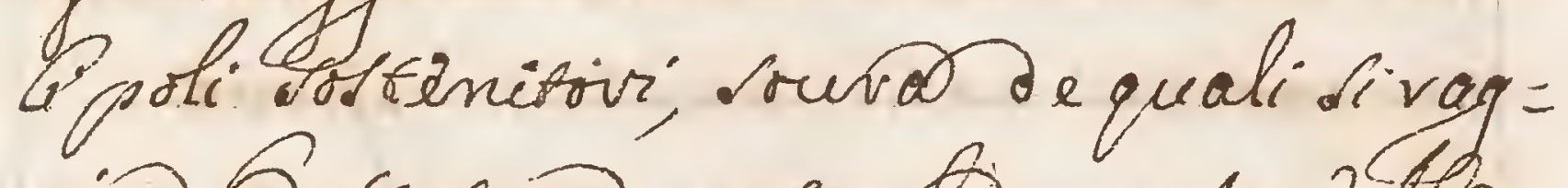
givabrativa, certo che acrescevebo Semine pici'sipoteña 
Odi nella Rofa il Miamerro formandoli di maggior es bendione, el me: Dedimo ne uevrá isarim ad angrandivis

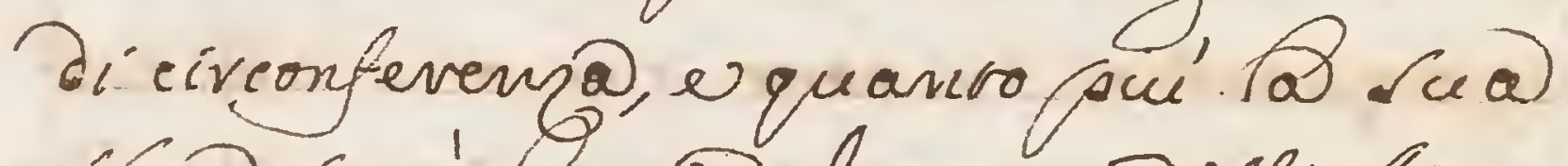
ffera ava' lungiral cencoro Dill Agse, ranbopici aquiolavio il moro alta vofa

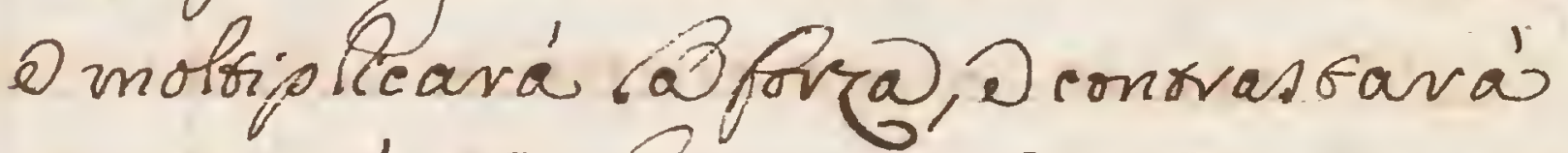
Panto sci con to materia da mount;

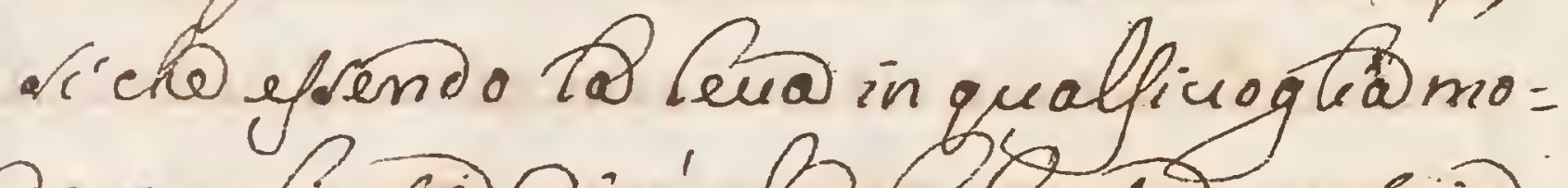
Do applicara, divo', che á Gusa motico nelta medesina, quanto vim arva'psié ra Angiral centro A della gracità - enja aluén dubio panro su' svenoe = vaballarolluctiono etangola B fov=

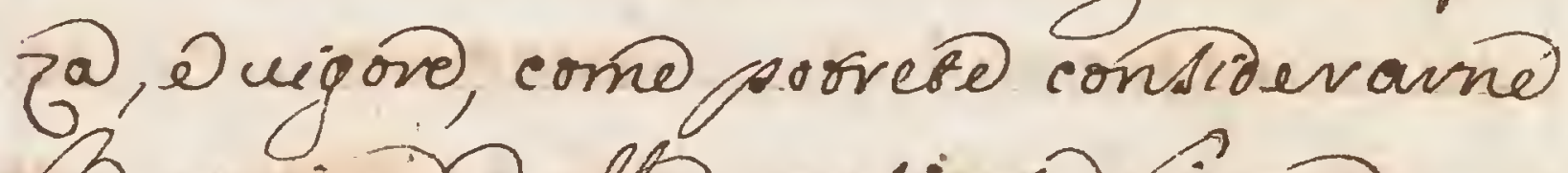
b cagione dalta presinbo jigura 
64

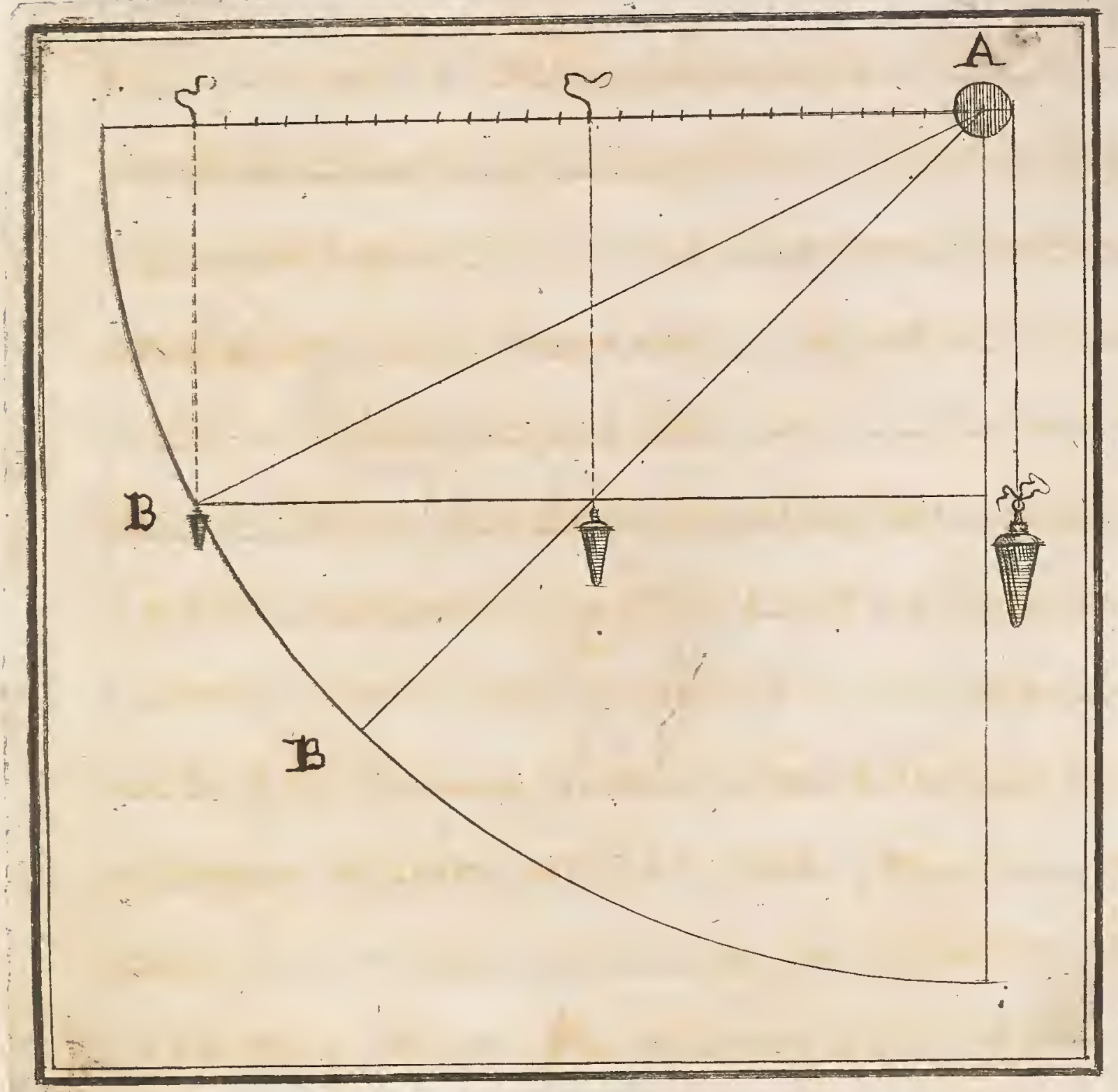




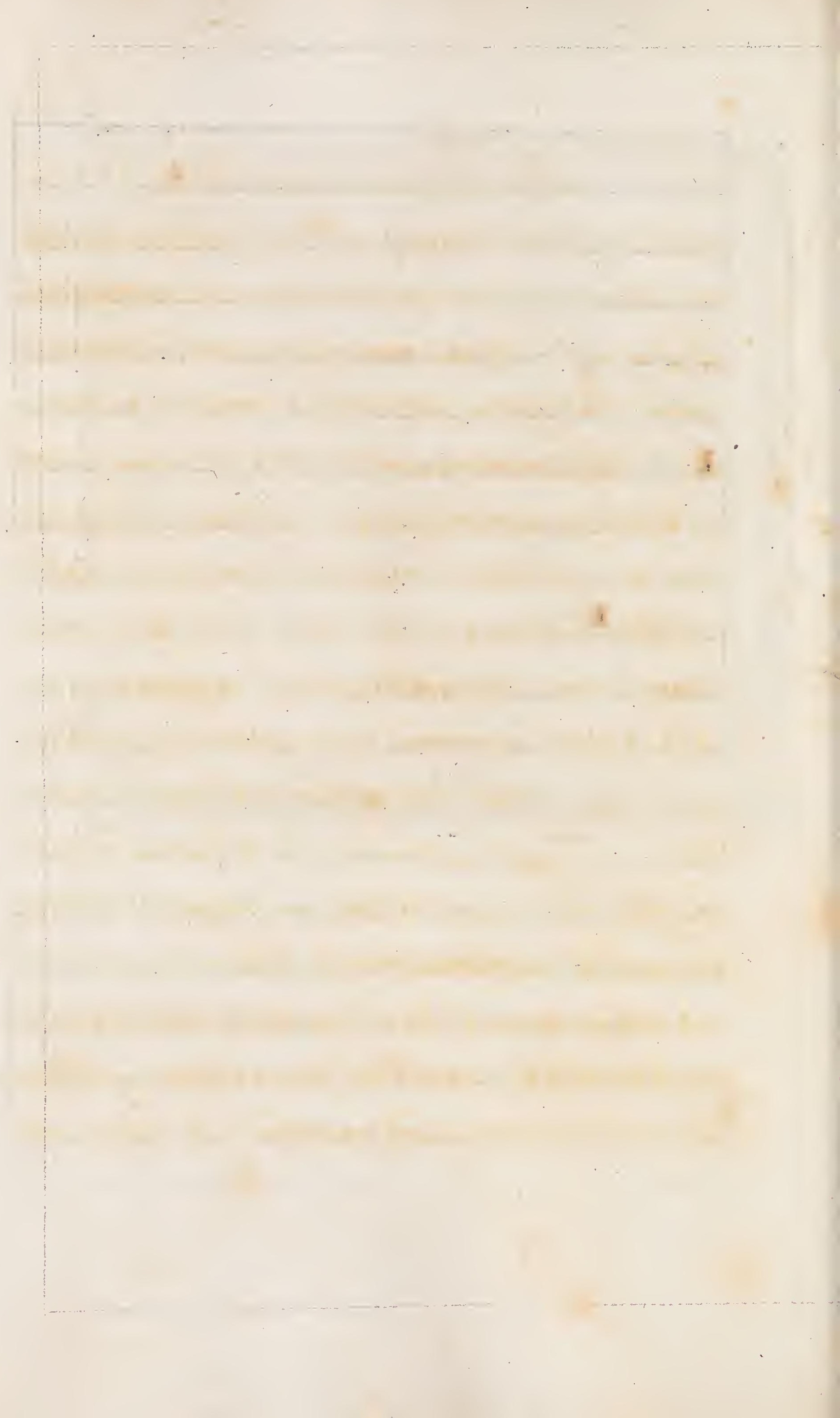

8 
6s

Mámi concervásiruiquale? colameora incorno at difegno, mevei che mi ono compromefso ti coecare tute quel = B. vienge, chesuono appartenerstiadun. beon Srofefsore, cosi sava' anche giupsitio che del Sopranominato jyeqno ceneporti qualche partictaritá je condo evodo ro. div' cho dinedesing hebbe Porgine dall: eqüto; do uivamento now ho apsuto in orracciavne unvprineipsis, ch' appaghi, nul = A 8 meno aceordandomi siv non fallive. con 'cicu', bivó con questi, chegl' gu foílio Dell accione, rante che rimouandol:

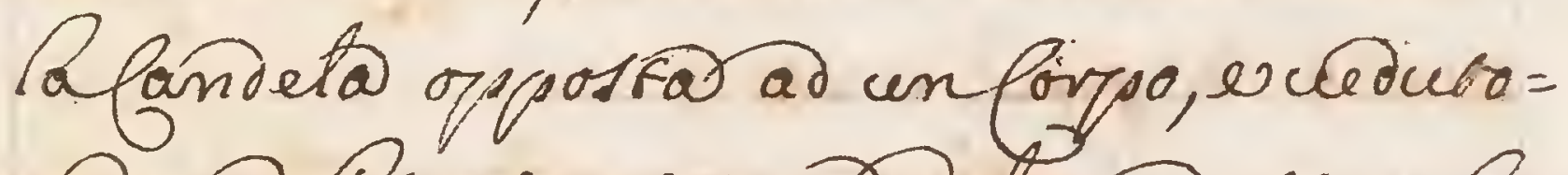
neper Pinteliposisione del medesimo or jograil muvo, 20 il cumo, frombraim. fovganella pariebe, inuaghiosene ilca= criccio ando eon un cantone in mano fú 
Bridesimafguradiquellombra Sineando,

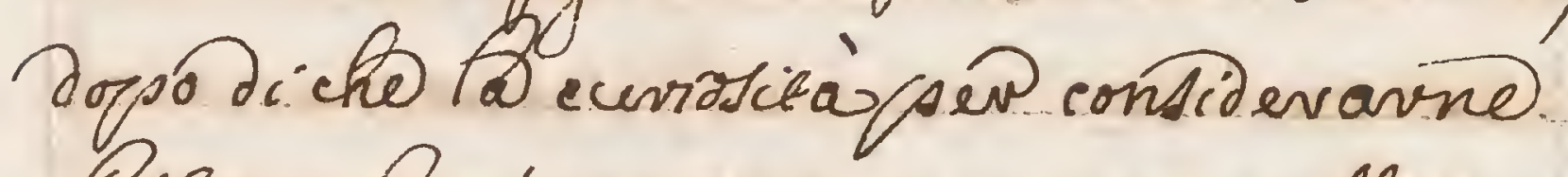

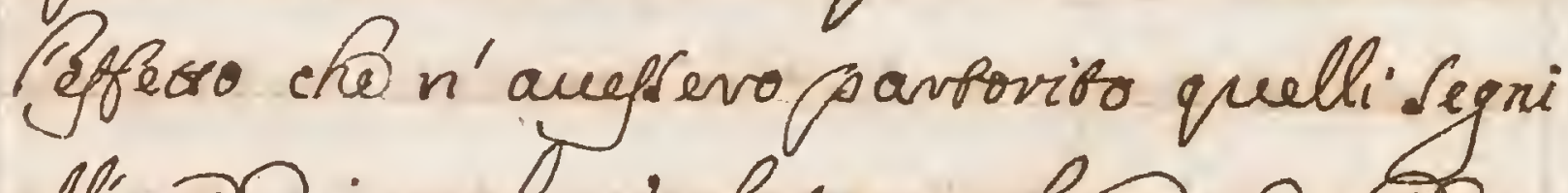
allion a mal rigolaro carbone ne wive quadi in aborto nato itridegno, effigh ique.

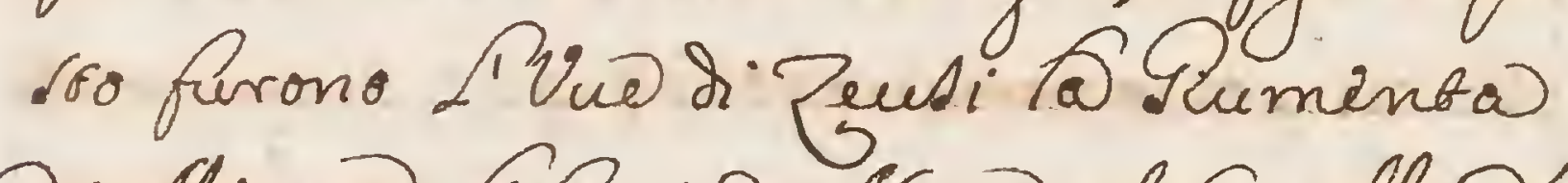
as Mivone, Li ani Di. Micia, il auallód' Apelle, e conto mivacoli dell' arfo Ca Venevd d' Esigmalione, o queltad S Pasdi: Git, Buagherze delle quali, o quel com = posto di meracuglie, ancorch or freds: marmi non cagionorno minovi incenay. chetavealiza'una Gellezo impa = satranigigli; viso

Aroice ovinohio, o Jalifono coura id disegno non Bytemprarono Lac= che, o cinatima li content arono ceu =

vaminsed'edevicicarlo.senga colori.tra= 
66

Eo, Q Refanter a fuanto principiovono a' byfringuero i colori coll' impasoarli une= doli afsieme, epoi vicomporti, D Vijybin = guenti in divents oiner, a cuíne compar= (i) il wivo nello piguro. Apothororo pers. facilitarna Parfe viforio Pinuentione panto necefariab@ porem difbémbeno

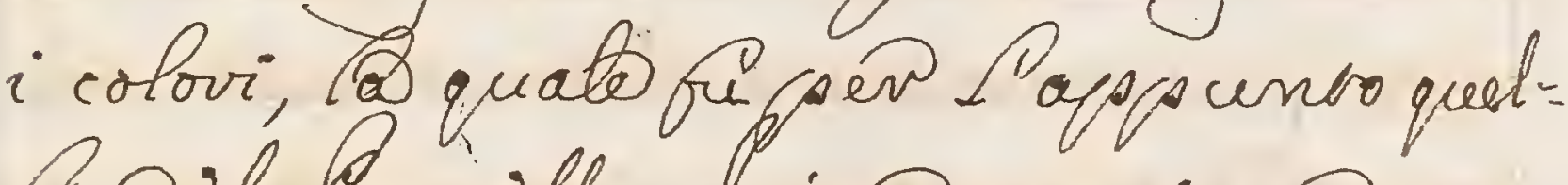
arell Sennello. gione per.ropiono me fi chiamato. Monoevomadas per. ciócho con un Solo colovo jipinde, máfre il primo, cho nelle sad figurd Jjgin : gueploil majchiodallofemina. 3 inone Corce vinuenne Acorci, mosoro $Q$ ueno nei' Orppi, enei' panneggiamen:

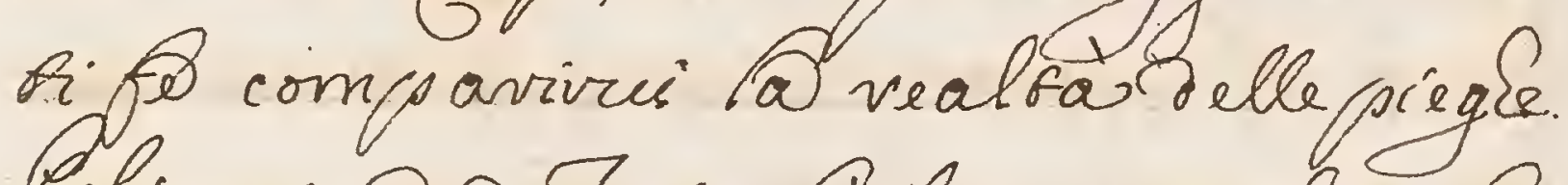
Solignoro da Taso fu' il primo, che $L_{G}=$ singafse@uanita' del sefso feminile? 
conch vifiouamenro zig ate ne gl' abioi: agguingendoui rello farzo dello inuen:

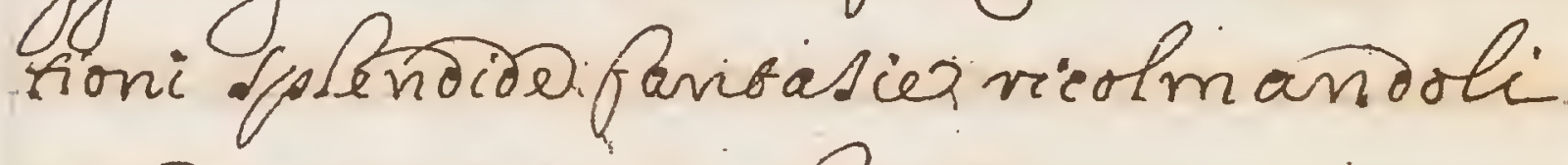
sor dicuicaciftimi Lumi.

Ton accadevás chís vidica chil avi Guti ingannó gl frugelli con Pvie, che forrasio deluse fif repo Zuati con un thelo, e chido 1 . manbe ancho Lavrasio fir ceinso. Meta?

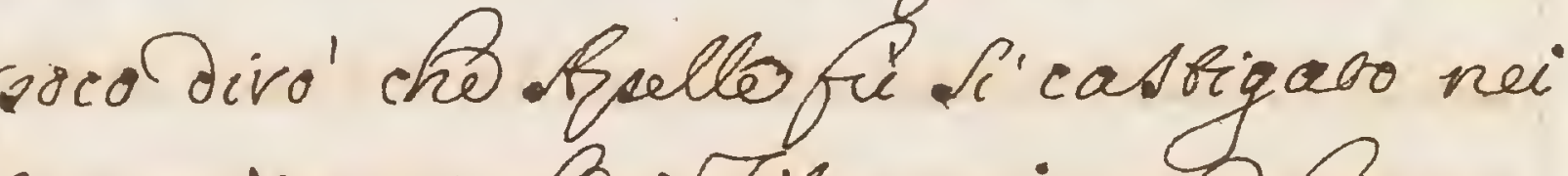
suriviratsi, the i fifonomi non forma = uano progusosici niente meno Dalle entone delinearerdal wredesino chedal uolos vibratto. Eufranove conservo' de": pre negl ciroi illecoro, Anfione difpo.

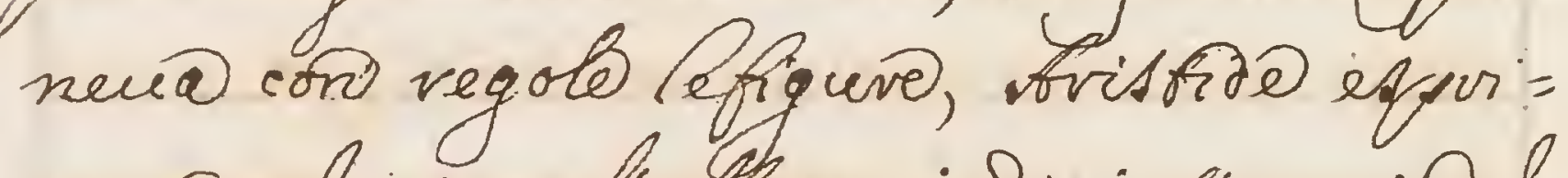
maxal al vicu gl ets; indipi sinceri del= le colfioni dell'. Animo; Ascriplodoro 
67

fir comentato 20. Filles per lo pro = corbioni; Autia Leña alfevacione del: Bparbirelineaiacon pantocivolpet = rione ovi beno i bambini seorqeñoruif: chiaraninei mededimi Lo lempleci=

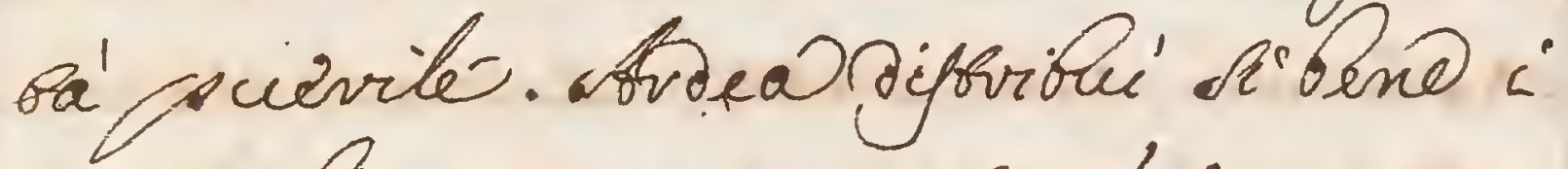

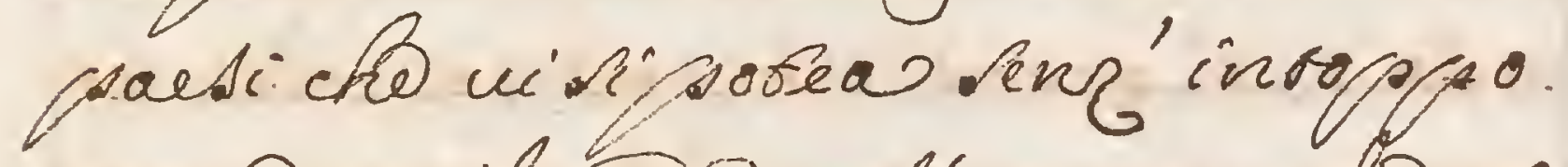
- non con il aclede paftegiarrei at ar: una col sentiever, bovto il cuanto bur:

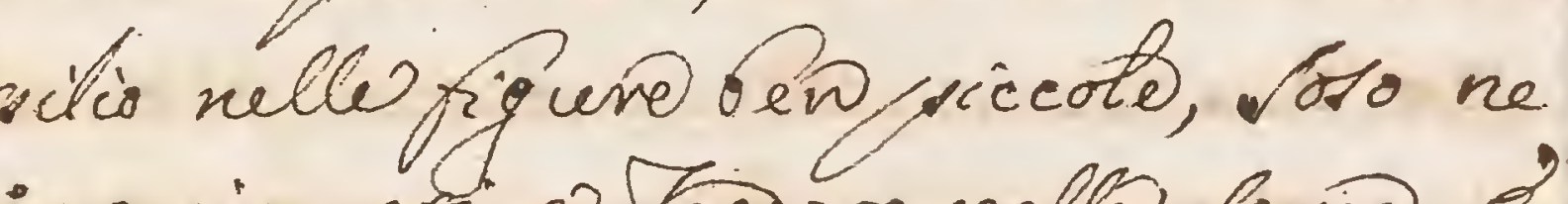
ipauimenor, o Teodoro nelle seño; $d$ poce cicie' ra a cingi del decolo brafeorfo Porchio si irysi nello seneverzadel Bregqio; nella ciuerza ciben edpref: - velle restre. 2. Tiziano; conde in? Arovea del avbo delicabeza; el of sivito del Solsano ne gl' Anima =

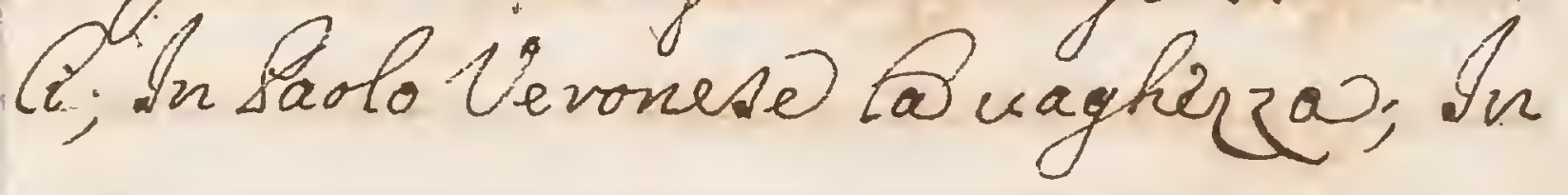


Albevo a uvo Ardiligenia, et al con =

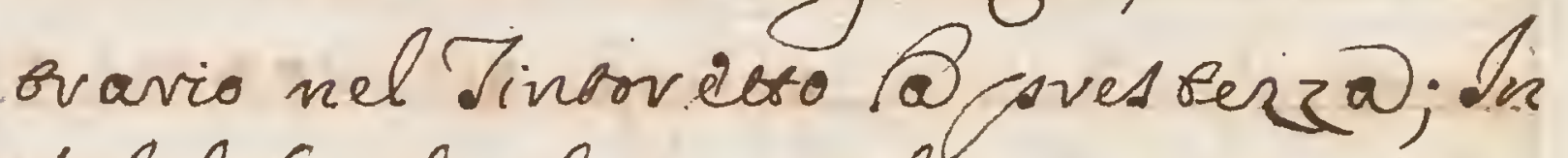

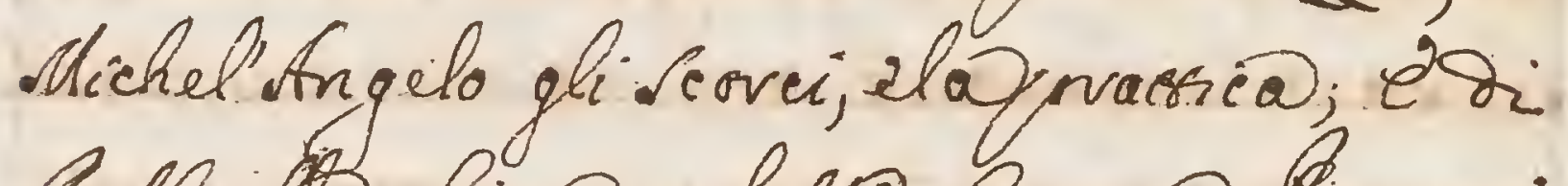
Raffails chi ne colefso foum are anegi = vici, Plpenbli beno grandi accuevreb be= vo.impro bing piceote á croporsione della.u@groñó intelligema. Bchicuo cefo carlañ on Pistori a'noi pici cu'ci: mitivebbe che aracci ha impide. QP cira' h. Buporr como Gbochlor appla: usi, e riquillidella Loro suota, chi non ammivanelle pele h. Scido Reni Tideo colorine or Savadio, nel Pome = michino B grazia), o to penfetsiono. gafrigat@nel Jifegno;nel Gueveino \$ for@del colovito; nell. Attano aroeli caserz@el@bizarvia nell-inuntione; nel anfranco lafailita' licuagoneghi. 
Ges

cuolazi de ifonni, choguivaress ino quelli efterric impriggion abo il uento. In

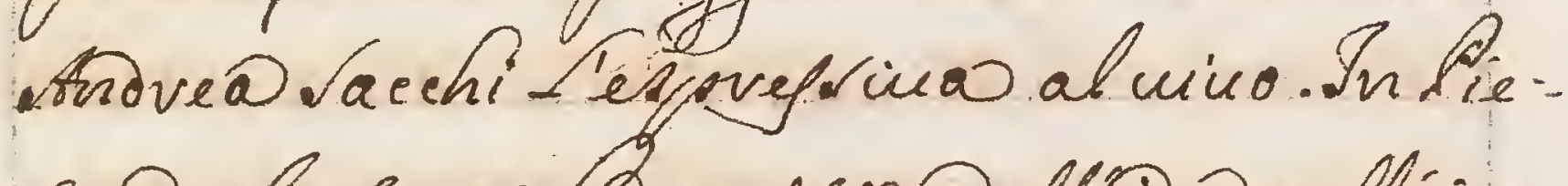

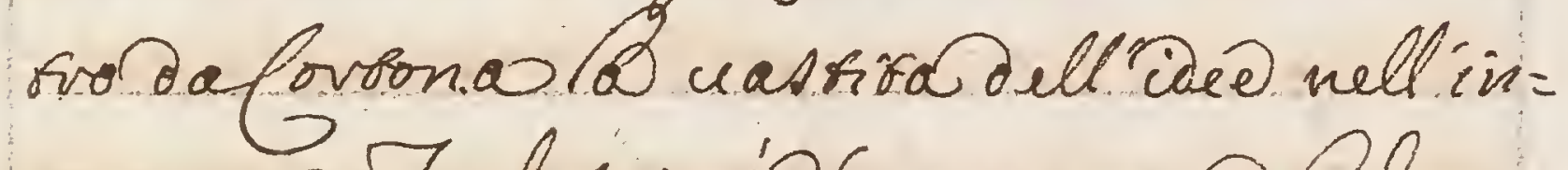
uentiono. Ivalasciarí' annouevaño alun =

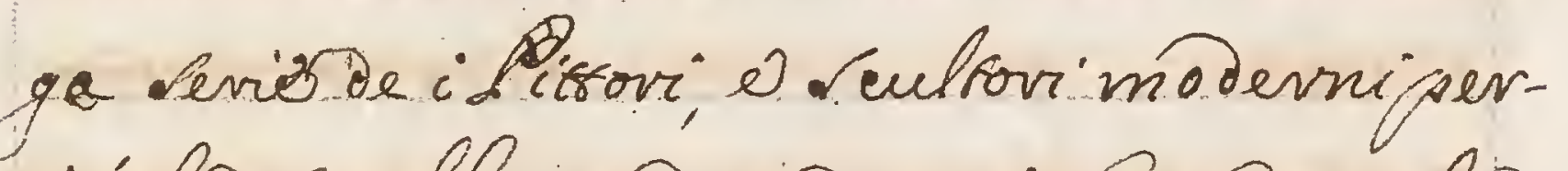
cióche arveble un nón mai zinive perla pran verio che neha podocta el nostro recolo, mà non uno afciarno incierro. it Bernino, chaper concepiono un con = ecoso uquale ot bi hi Sapene, Gastera's che sene vimivicl Fonre inakavo in Mas uona, rob nell' infreccio di fante inuin= Rioni hi capvieciofi forami nelle vochure ae gli eogli, como ne gli scovei siben adow

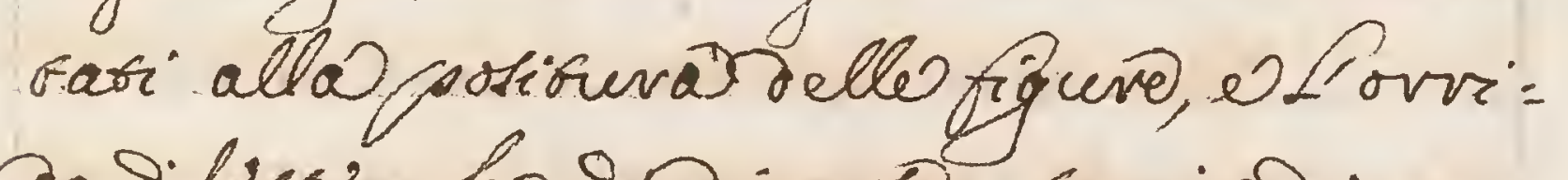

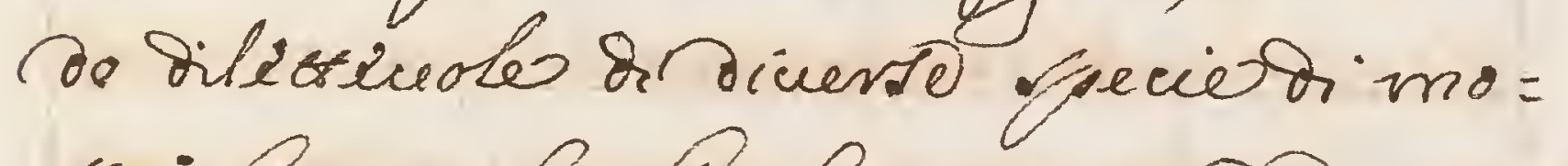
revi fanno cho Pocchio w'digerval come 
in un Coberrinto di Gupori:

Voglio ren suggenive, quando

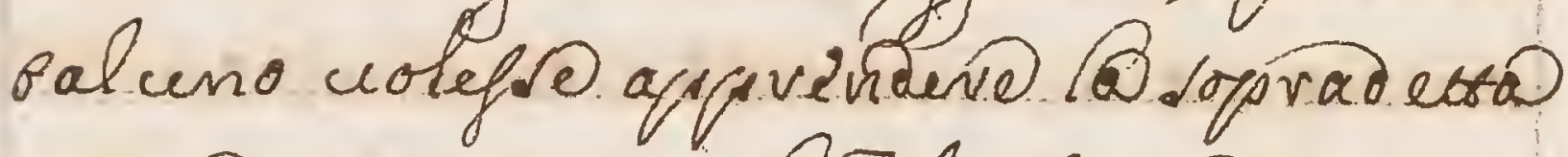
vienz@nowsendo eglibilsofonondistin= quevibbs accidenor senga qualcho some D'errore; 'egli non in rinderià a' Anora = mia haueva' or bjogno del evusico, cho giviponga fofso al suo Lugo,inevi nel suo sito che il pennillo Fauevibbe forfe?

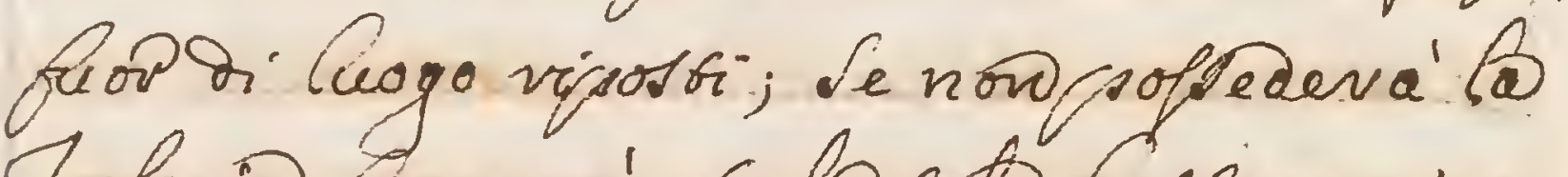
coologia formena' alo beto besfemmies aysinte, come in saluno Sen viernobb $\mathrm{c} a=$ sivico fistrovo biceño

Nonfaro como unval Litror a beno.

kie few un Rua, equivipinte un filo

Aep non far appariv Q parki osene 2 codi covregendo ancho un parienore

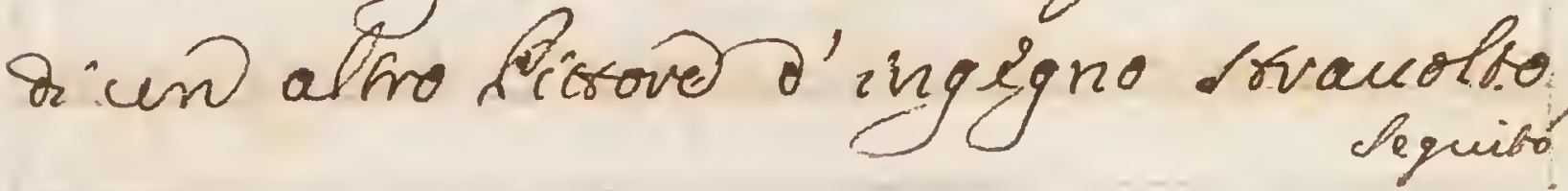


69

sequitócon Aistejoffengo àio

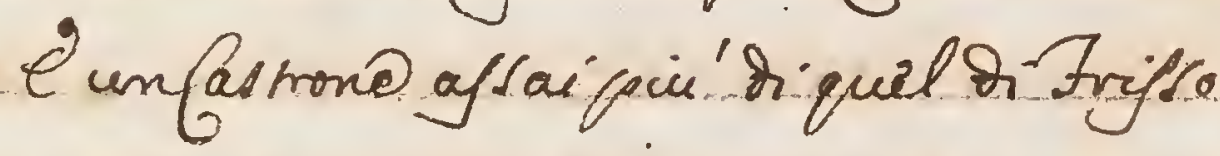

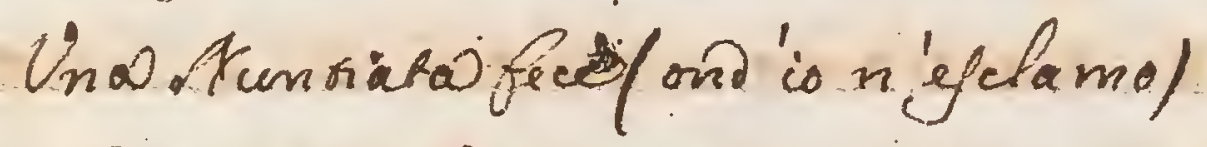

hericu@ Ptivio a'un rocifiss

evisigliano if medesimo fir del nostro ajcorse se cl firsoro nón aueva' bratcorto 4

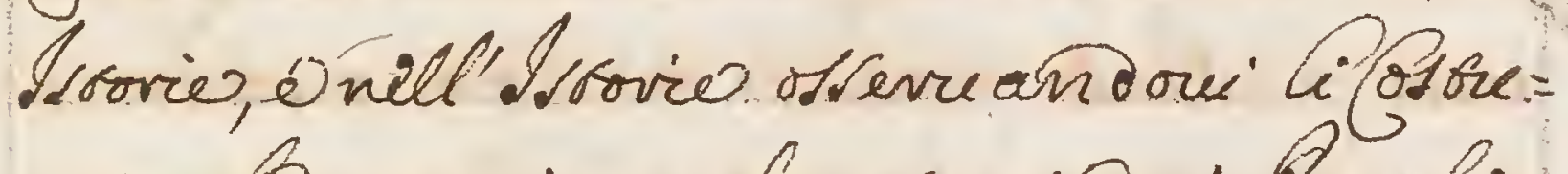
mi, ele propivio inclinationi de i Aojoli poura' con devisione th So sefso forte un abivo greco indofso ad un Lárino. é nox rarafsoebafsvinotra's Jaglis in vap. = presenfaño Iŕnuentione Jelle faciotono

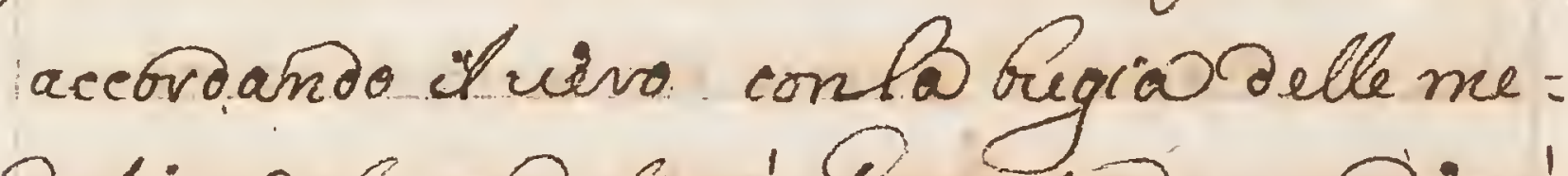
desime, venon avá Sémerí pveñeva' errore nelle proportioni; Se now Sava' Arvkifeto now poova' an comparive incen@sacotai a' quifor incanto fops si, Arehi, OBeabri le non sava' osmo. 
grafo non a arrà vender vaggione nebam

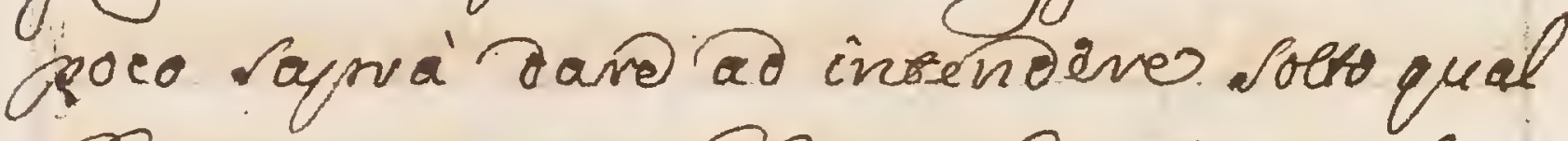

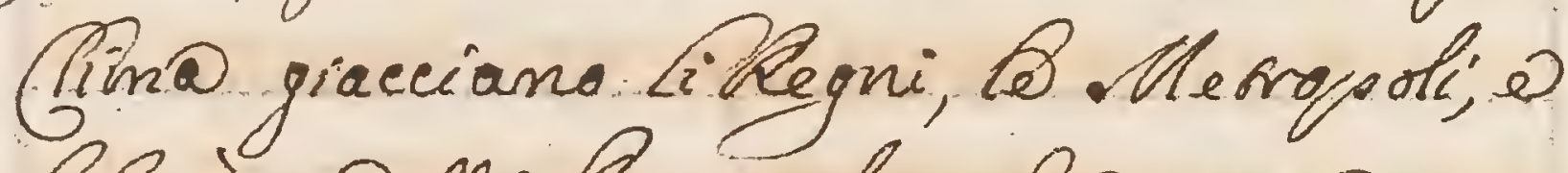

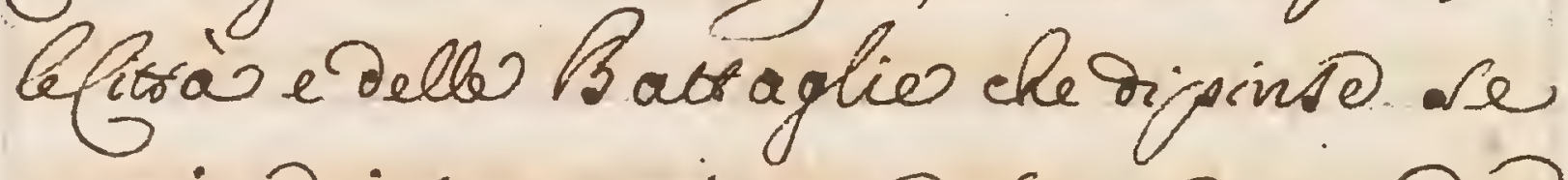
me ciene interrogato non aypro verses? conto bel sirs ou no accadd la puga,

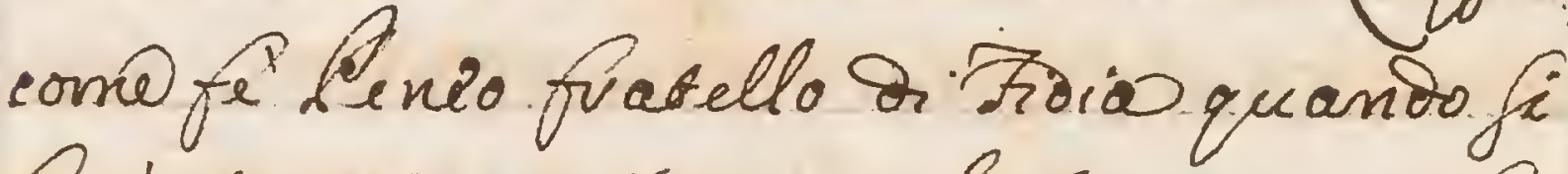

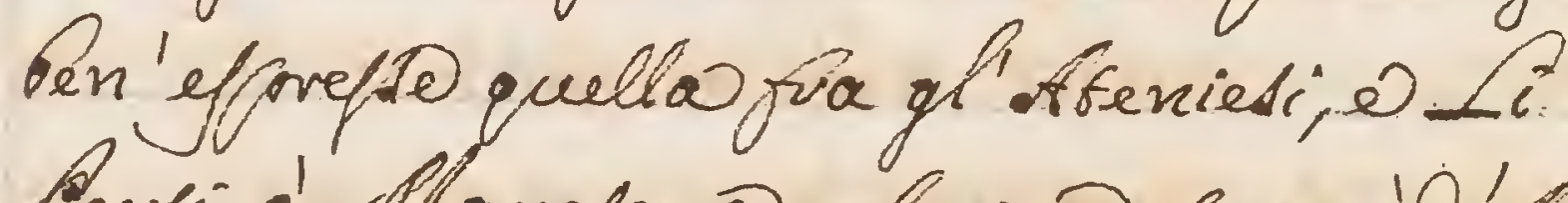

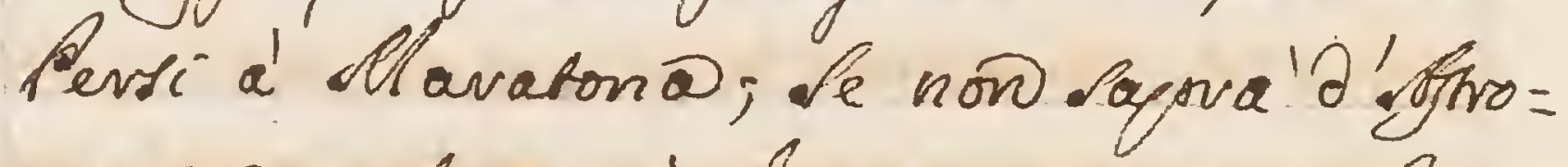
nomial confondeva' if propsrio grado che

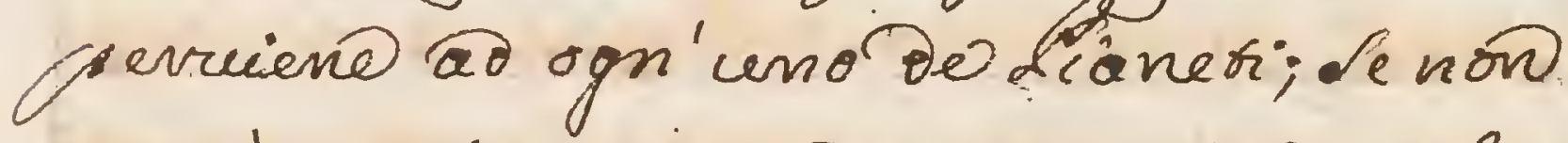
-ava' mosperico cader amnoda celbs: lesuógigue, precipitarannodogni momento gi oifici, ancovche Dipintz:

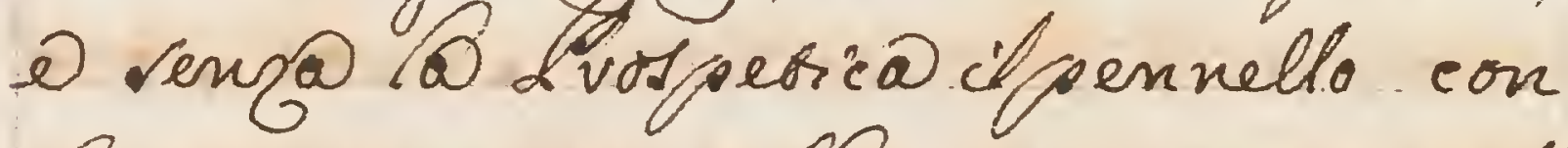

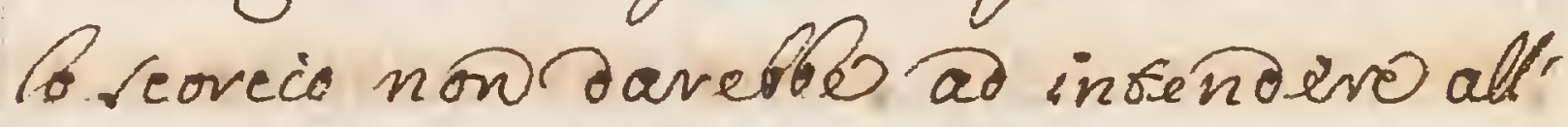
occhio quel che non ta. Mlate preande:

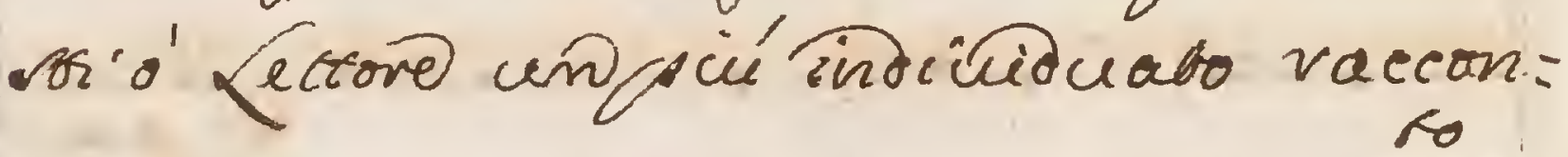


70

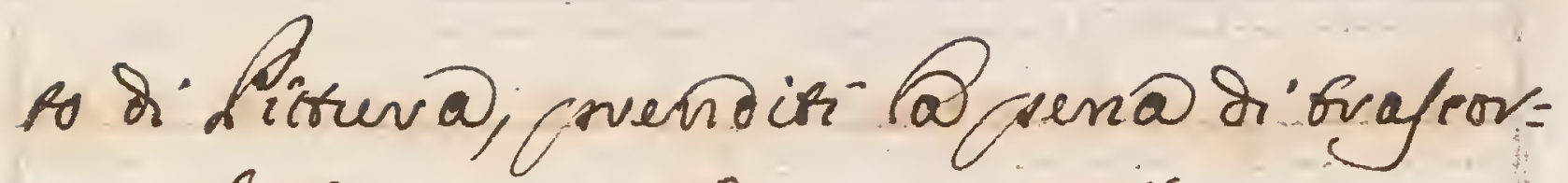
vero if Vaf avi; e fe fram of tri delle partico.

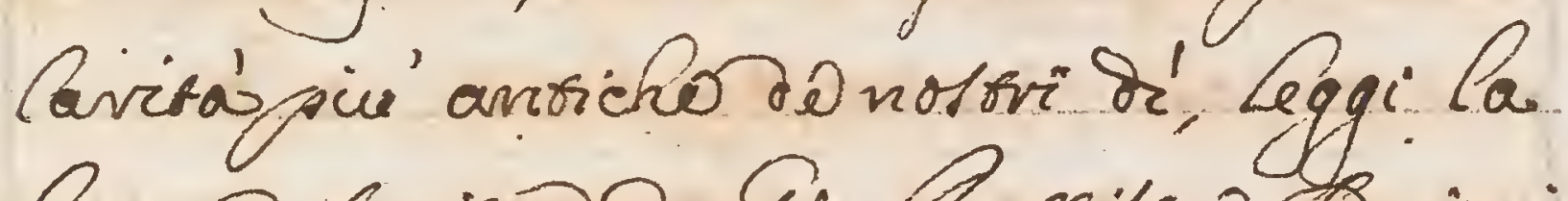
Retera revitrara Gio. Sarfíra Stoviani all'istefso Vafarri, cho vimarrai piena : mente informato con "aggcieno 0 in un numivo infinito $\partial$ 'alme evarisioni

- M' nell' aveñ is bijcorso.

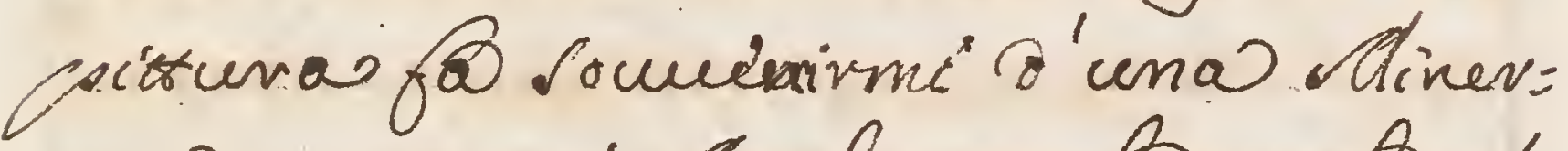
uadipsintoba' Afmulio, contaquatem'. apviro @rada a'Jivk quahehe partico.

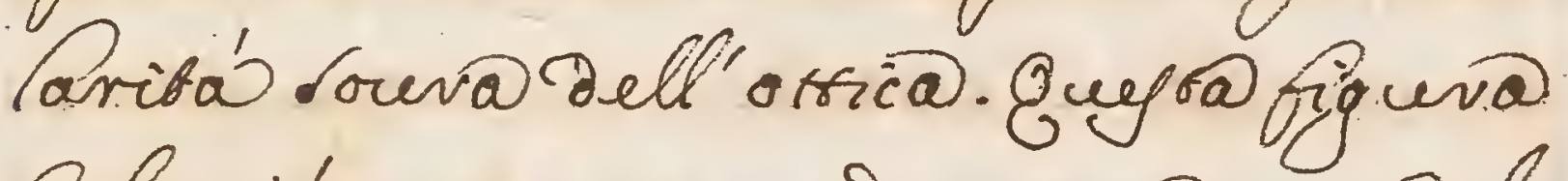
fulapiú ammivaro di quavo no deli= meafse il pennello Latino, of arecea. egli contanto artificio, e siben accommo = dẩnill'occhio Ofsupilta che ragiona: ua von meno duy oves a' mivarta che effernorallomedesimaviguandato rici 


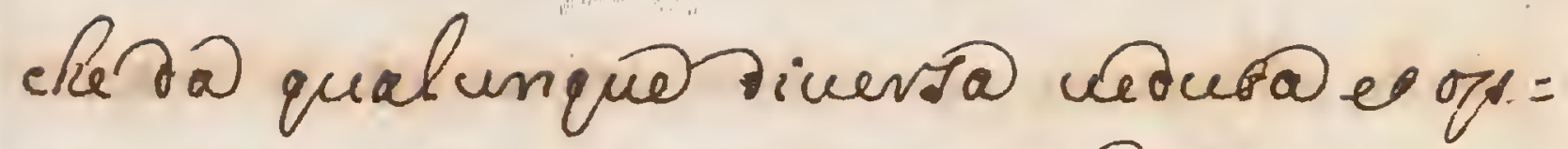
cosfa carbo foro vileduta ben sorgeua = si quel fate viontracambiassir allo me = dedima L'óguândi. Airemi ac graniâno souniogust.finulio fiaces ap prefo

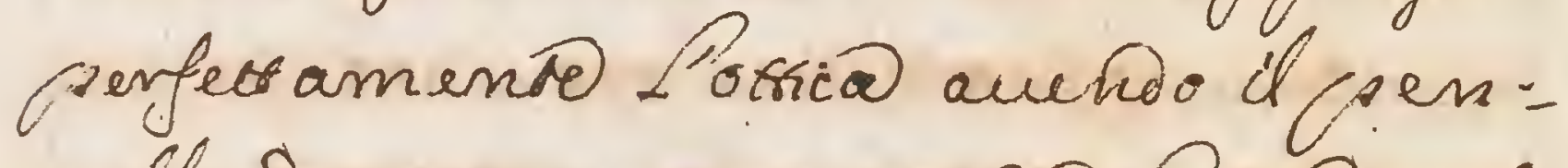
nello Dipinso quano jogta hauen enfi: derato conla rivifionerdelle sasti Ano= romia, quatonn mertimo ingagan = Do ivaggi vifuats sa gl'ogg etr ujibili in maggiove, minor numevo, econdoche

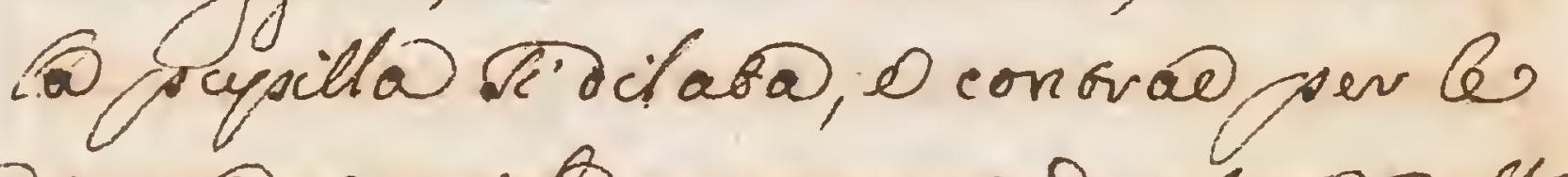
Dicuense funiche, et umori hiafane dell' sechio, fin cho í frouino leloro peits ure to immagini infsofibure solo vicuente, rquifitamente collocabo vello ounica?

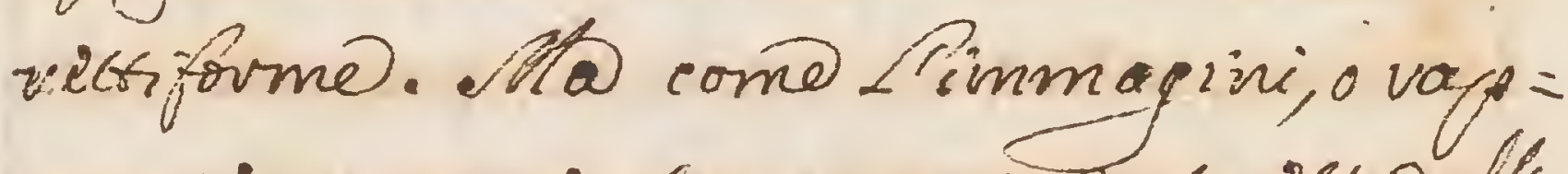
curesintabioni siano por Propmefses all 
76

animavagionecuote, w aivebbo frovo Arnu: is che cio fija slo col mezzo di quel nu: mivo increoitile o' capillaminti, o filess: che compongono if nevreo offico, come an

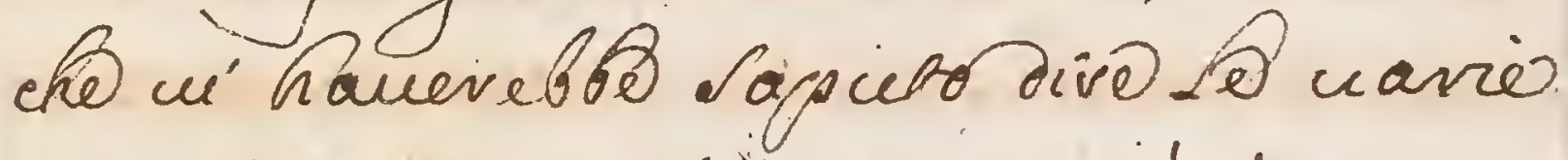
seveofs fatso nelles estrencitás diquegt: capillamenti, es che nello vitino. c com = municano all'alwo es ovemirábellime Defimi che ferminan nelferrello. N'aue= vebos ancho Amulio insegnato come fotni= mo contemplando Puna puózjeernevo aneo 'altro, condiquan uariéba' ingu:

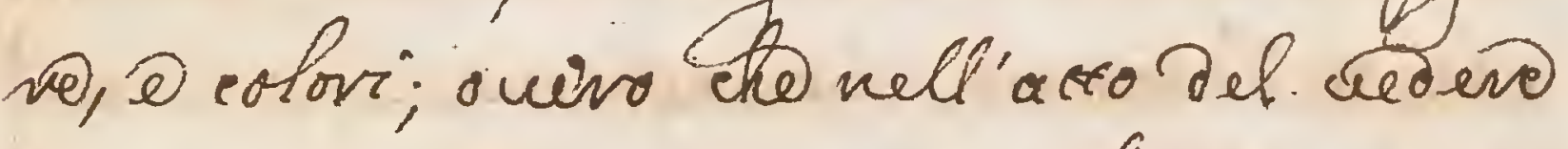
siseviedallisiviti animali (quei mev = curyj ulloci, de qualivicatio infuon nu: mevo ni cunfricolid el (evruello) coer por = fave lespecio ne dur pricuri issostigli, uidivebs anche, conde. sene serree, eli 
mand@fiori in maggione, ominor nume: vo pis animave i muscoli, es in quefto.

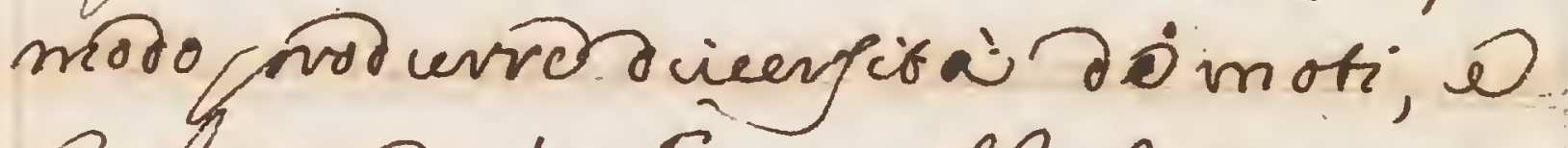
pinalmente u'informavibbo he cosa Siano quefri sivitelli coli atsici, cond uingano commandats; o come ofijjcant; ma $\theta_{2}$. "io' intivrogateno il medesimo, occero $Q_{a}=$

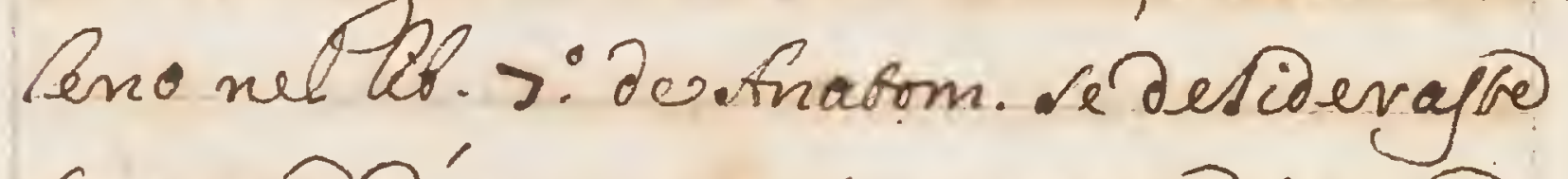
ayerñe d' auantaggic, mentro is now -vamo hifarne alwa inchizfas per now effere maggiorm enco cungo nel mio dy = rovso non aven ond aucuto al wo vifleglo

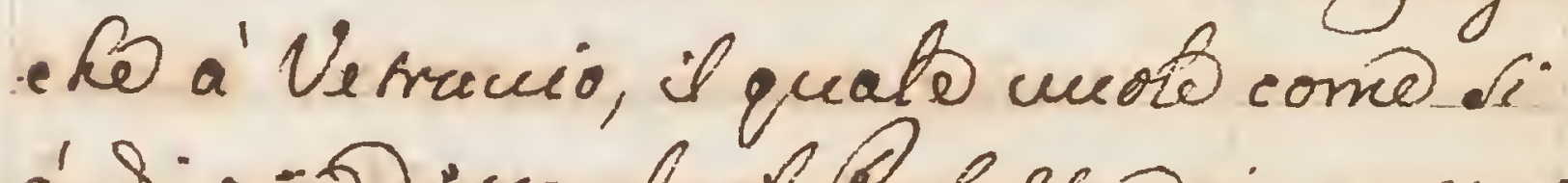

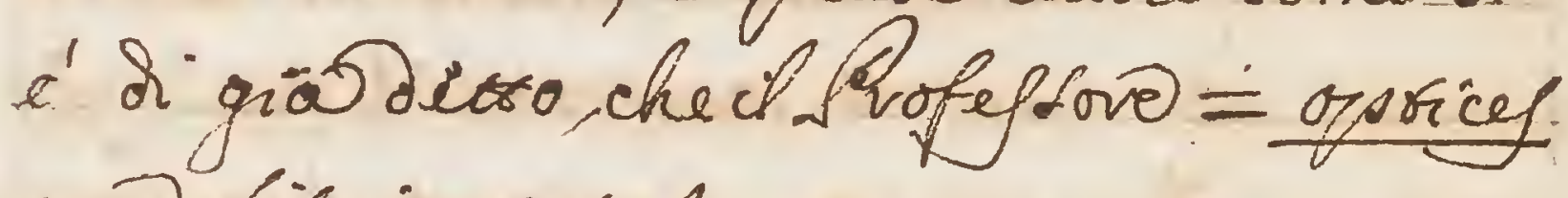
now dizignarres

Mivisveñdevás fal ceno che so=

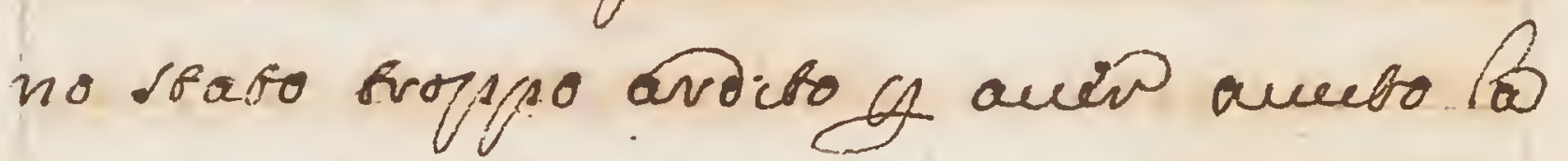
protuntionedi eveare unia nuoced inuin. sione 
72

fiond, e ruas andareté approuare. Rifjon: doch Olachina Di Aomenico forriana ou un'impresasiervica ckehafouto far. civesii J'un serolo, maquefa machi: n@ysendogia inuechlabanow viporfa alwa raccia se non quel biafimo, che acqui= sounabelfá, Oquate quando Se'fao = ta commune, ouevo acenzatan negl' annifsord íncor Vans:

Nazi@arebb.red Mondo an =

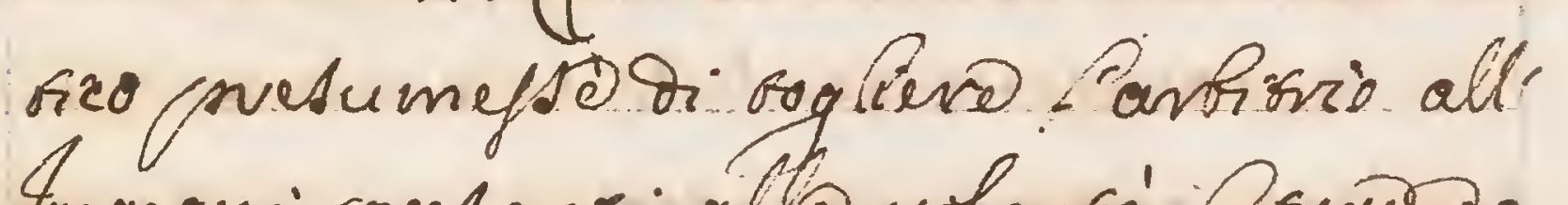
Inqugni povetensi alle uolonfá furend da now soren proburvall des nuove renza añ avio elemojinando dall'ebá Rrasandato Pincuntioni. Pisteftaon = nissorenjaliuina,no pur ella r'e'vi= Levora. Putforita. ouvardeno.=

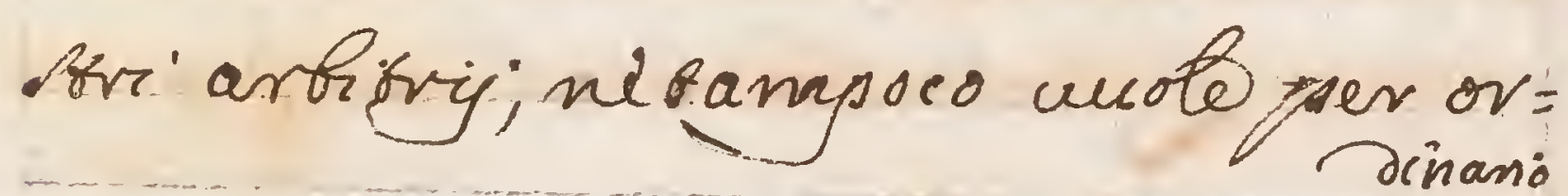


Dinavio opsporsi à gl almuiliolerr.

Lo gevo nonm arrago ban = foch pretindacomo. Ormogene, ilqua: Tocon un intendimento eccosnre pess

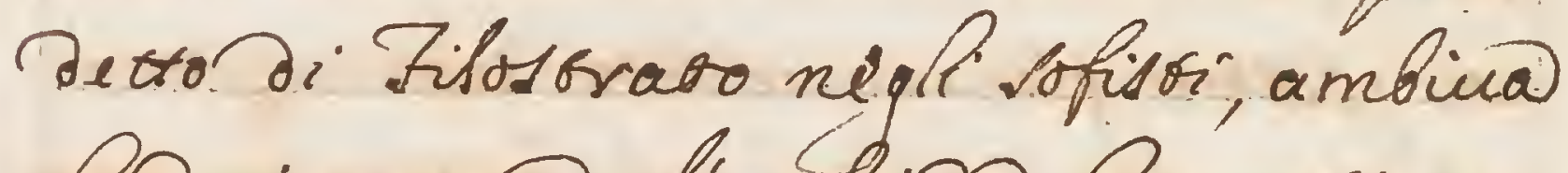
fap vimanere gl occhide Leterari am = mivanos outto cio' cho opevacio, dico. beno chopers quepar incuentione che lo horato allo Nce se nôn ne suc mi = virant Bos combunidea bi perfectio no, neb ampoco docurvi aspectarne il biafimo conn indagatover un nuove modo' operavo Senon ch altro, almino a'viftejso di ricto eco cho quial dis $S_{0}=$ urainmiajjets@g quanrobal nio pe fllo insendimento mi e' frato permeg - ho navrabo

Pimbecitliza" Del mio ingegno 
73

auevebe ambito d. fojfans maggin: mento o' Letrove bencho non pof afsicurarmi d'auention quisto oviue vacionso á bastanja spiegato qua $\vec{a}$ to 8 uolonbómich = Caacua,mal do. eramírufara'. con mive?

1\% detino cuive samen es budenda uo = lunag 



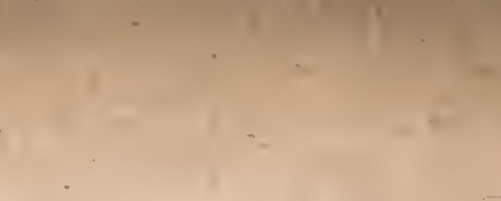



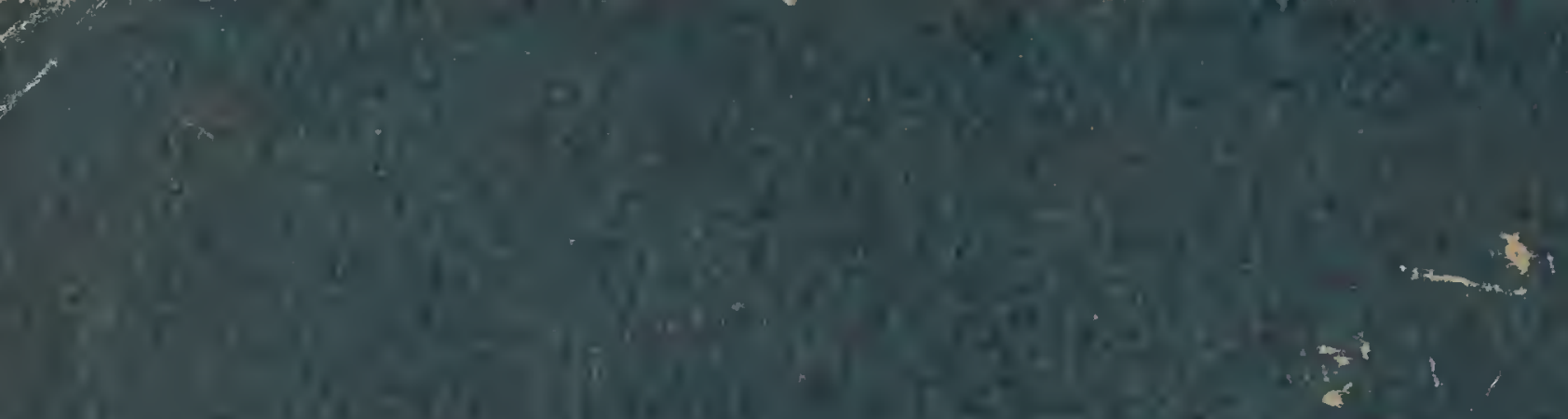

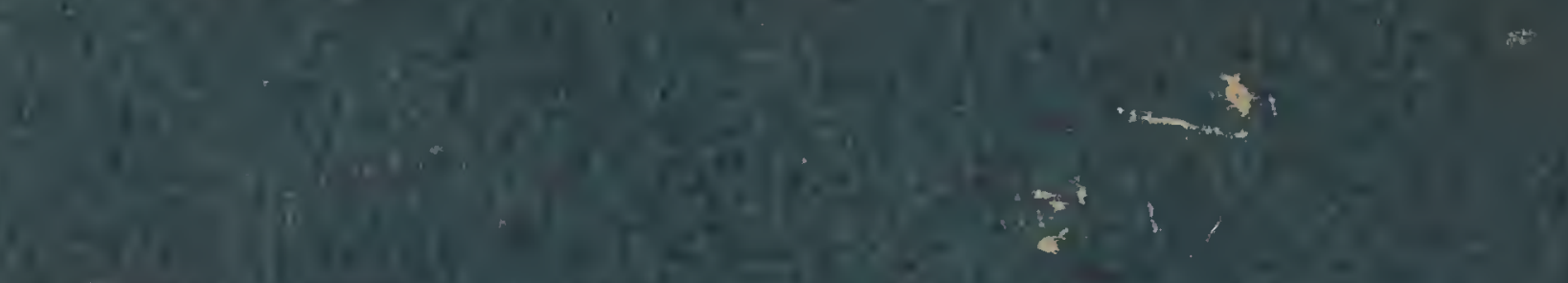

in. 1.

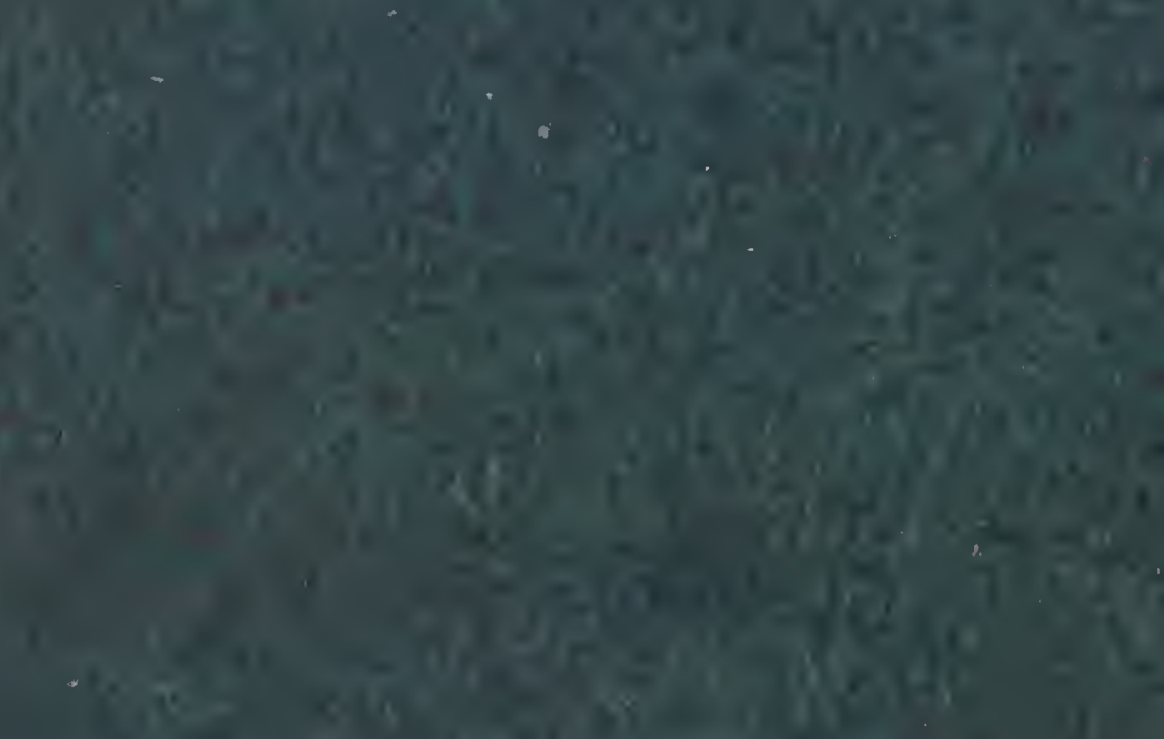

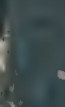

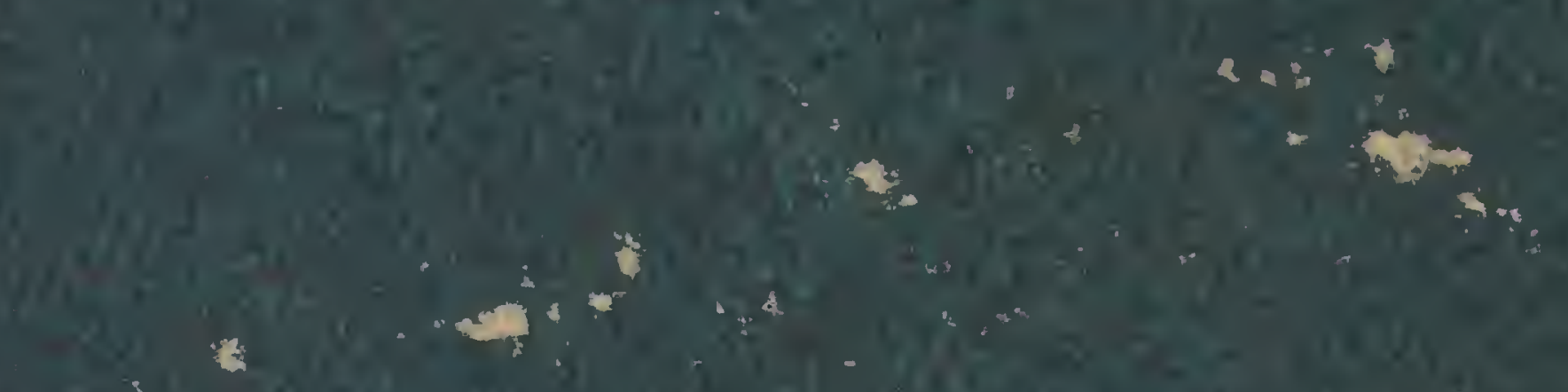

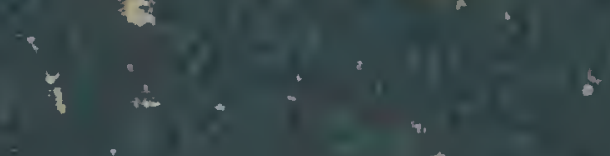

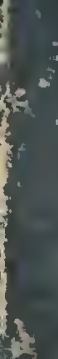

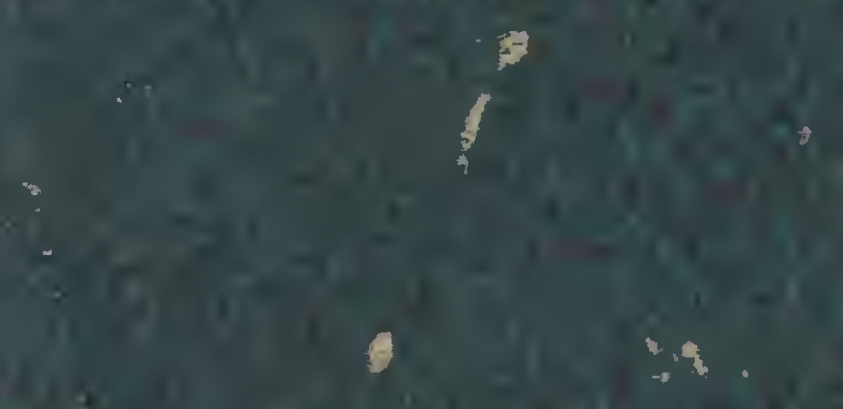

4

$4+x=$

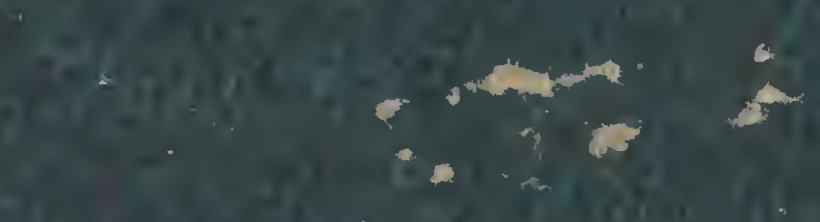

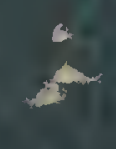

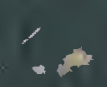



SMITHSONIAN INSTITUTION LIBRARIES

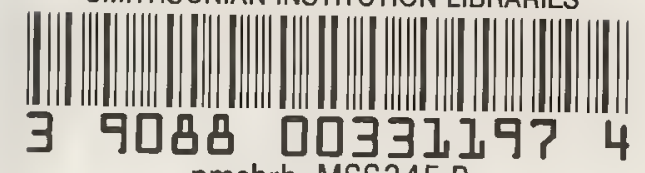

nmahrb MSS245 B

La forza della leva 
$g=\dot{B}$
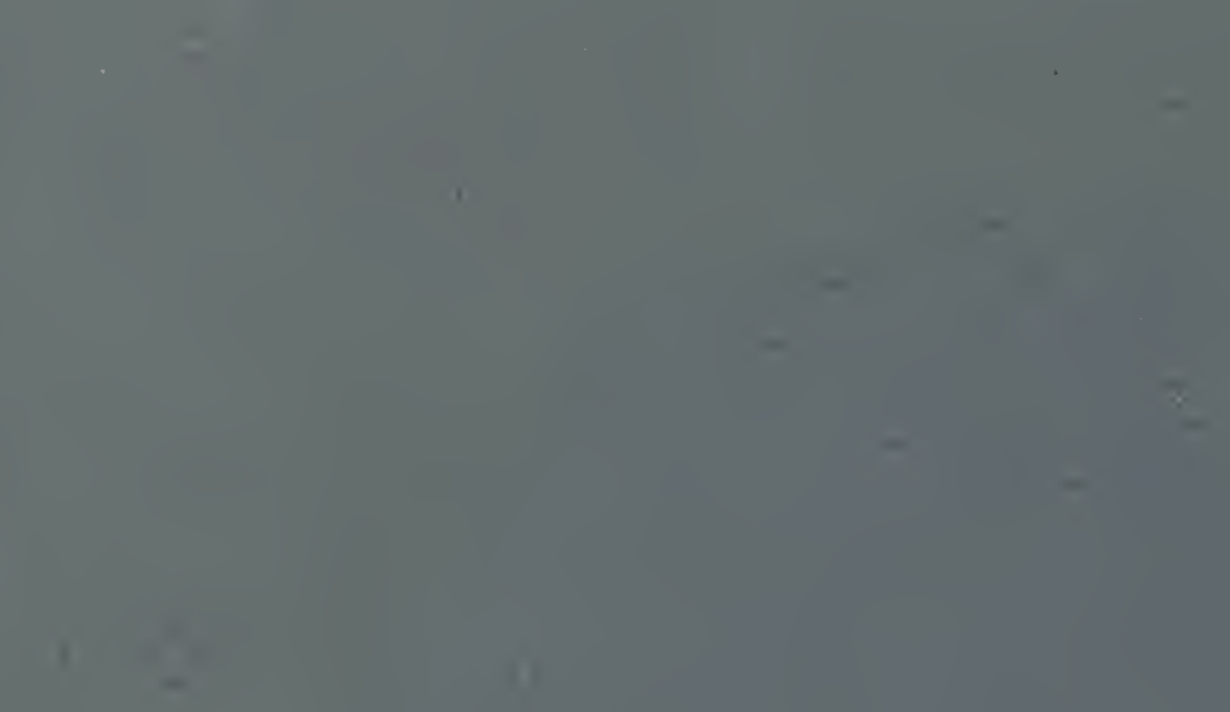

1.

$=x$

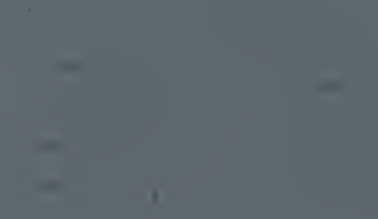

$=$

$$
=
$$

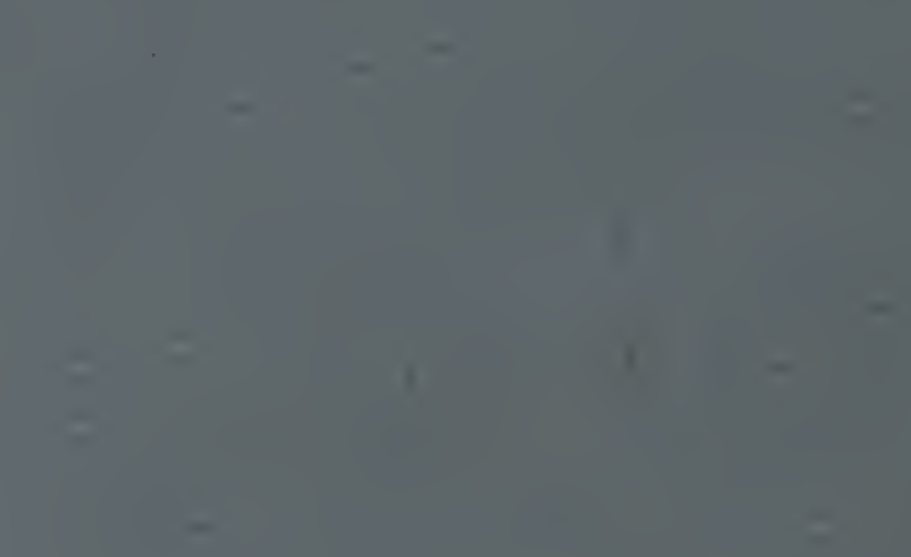

$=\quad=0$

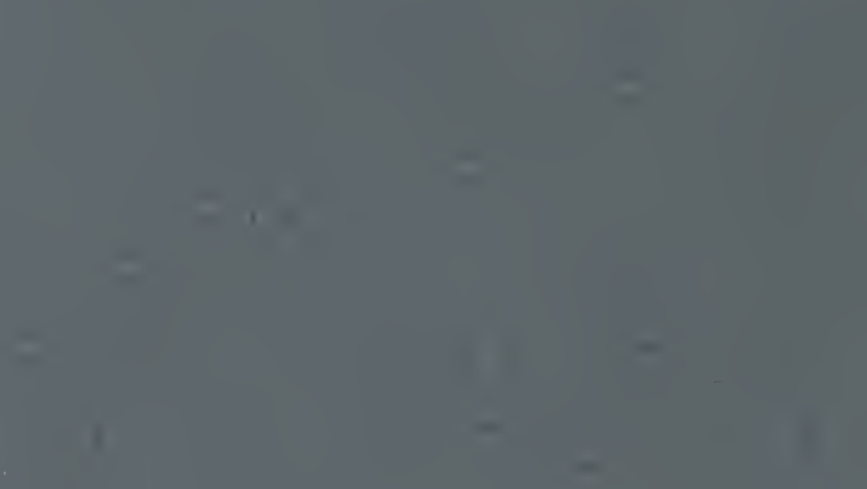$$
-\quad-
$$

$7 \times=$

$$
\therefore
$$

